\title{
Effect of Shroud on the Performance of Horizontal Axis Hydrokinetic Turbines
}

\author{
by \\ Mohammad Shahsavarifard \\ A Thesis submitted to the Faculty of Graduate Studies of \\ The University of Manitoba \\ in partial fulfillment of the requirements of the degree of
}

DOCTOR OF PHILOSOPHY

Department of Mechanical Engineering

University of Manitoba

Winnipeg

Copyright (C) 2015 by Mohammad Shahsavarifard 
Degree: $\quad$ Doctor of Philosophy

University: $\quad$ University of Manitoba

Department: $\quad$ Mechanical Engineering

Title: $\quad$ Effect of shroud on the performance of horizontal axis

hydrokinetic turbines

Author:

Mohammad Shahsavarifard

Supervisors: $\quad$ Dr. Bibeau E. L.

Dr. Chatoorgoon V. 


\section{Abstract}

A $19.8 \mathrm{~cm}$ diameter horizontal axis hydrokinetic turbine and two shrouds are designed, fabricated and tested in a water tunnel. The shroud is a convergent-divergent duct and the diffuser is a straight wall duct. Effect of shroud on the performance of the turbine in inline and off-axis flows is investigated experimentally. Consistent power, torque, and thrust coefficients for the shrouded and unshrouded turbines are obtained for a complete range of performance curve. The results are obtained for three Reynolds numbers of $1.38 \times 10^{5}, 1.77 \times 10^{5}$, and $2.17 \times 10^{5}$ based on the rotor diameter and undisturbed water velocity. Free surface proximity and blockage effects are corrected using a onedimensional actuator disk model. An apparent maximum power enhancement of $91 \%$ over the unshrouded turbine is obtained with the straight wall diffuser design. The maximum power coefficients are seen to increase with Reynolds number but at a decreasing rate. The Reynolds dependent behaviour of the power coefficients decreases at higher flow velocities and disappears eventually. It is observed that a shroud is more effective in low speed streams. Power coefficients of the turbine with the diffuser calculated based on the diffuser exit area are found comparable to the performance coefficients of the unshrouded turbine with the size of the diffuser exit diameter. At water velocities exceeding $1 \mathrm{~m} / \mathrm{s}$, an extended blade having a diameter equal to the exit diameter of the diffuser generates the same power as the model turbine with the diffuser.

Output power of a hydrokinetic turbine is observed to decrease in off-axis operations. The reduction is negligible up to a $10^{\circ}$ yaw angle but increases as the yaw angle 
increases beyond $10^{\circ}$. The model turbine with the shroud experiences negligible performance loss in yaw operations compared to the unshrouded turbine. The maximum reduction in the performance of the turbine with the shroud in yawed flows is $6 \%$ on average at the three Reynolds numbers. This is $17 \%$ less than the power reduction of the unshrouded model turbine. In an analogy to the cosine rules of the linear momentum theory, similar cosine relations for the shrouded turbine are deduced from the experimental results and proposed for the first time. The optimum power of the model turbine with the shroud and its thrust at the maximum power are experimentally observed to decrease with cosine of yaw angle. Based on the new cosine rules found in this study, the turbine with the shroud in a $45^{\circ}$ yaw angle would generate $29 \%$ less power than in an inline flow. The turbine with the diffuser would produce 50\% less power and the unshrouded turbine, based on the cosine cubed rule of the momentum theory, would generate $65 \%$ less power in the same flow. This characteristic of the shroud is expected to be a function of yaw angle and is anticipated to alter at higher yaw angles as the inlet angle of the shroud is close to the maximum yaw angle investigated. This study addresses the fact why the reported augmentation factors for diffuser augmented wind turbines in the literature has a broad range from $-36 \%$ to $760 \%$ which shows disagreements between researchers. 


\section{Acknowledgement}

I would like to first acknowledge my advisors Dr. Eric Bibeau and Dr. Vijay Chatoorgoon and also my committee members Prof. Mark Tachie and Dr. Kris Dick whom I benefited from their support during my study. My special gratitude and appreciation goes to Eric for his generosity, guidance, and his patience. His supports during all these years of research not only let me grow mentally and research-wise but also taught me a lot in my life. Interest and courage in facing everyday new challenges are just two blessing characteristics I learned from him and try to keep and grow. Next, I would like to thank all my friends in our research group and university whom I gathered good parts of my life puzzle in living with them. Particularly, I want to name Mohammad Saeedi and Mohammad Aramfard among my friends who were always supportive and encouraging. I would also like to sincerely thank Amir Hossein Birjandi from whom I learned many life tips and who supported me technically and professionally during hard times. I thank my sister, Zahra, and my identical twin brother, Ali, for all their helps during these years of separation. I finally would like to cordially appreciate the remote and continuous supports and encouragements of my parents that I was not able to get from anybody else. God bless them and live long. 


\section{Table of Contents}

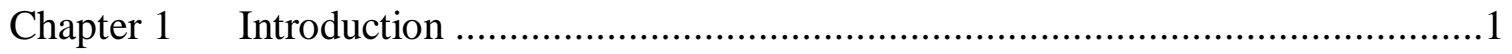

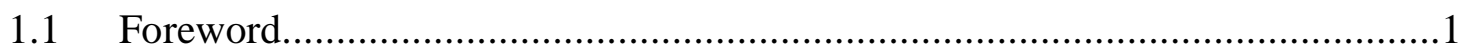

1.2 Hydrokinetic Energy and Horizontal Axis Turbines ........................................

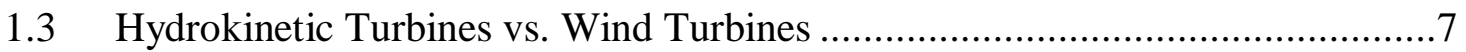

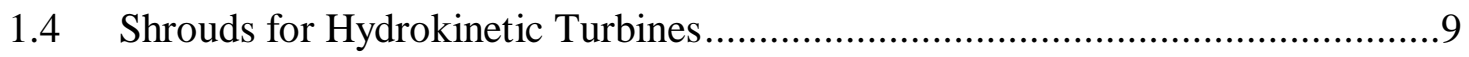

1.5 Environmental Considerations of Hydrokinetic Turbines................................11

1.6 Motivation and Objectives of the Study ………......................................13

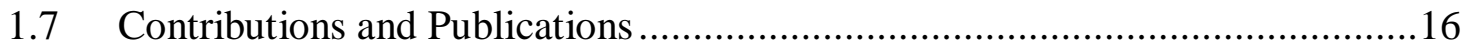

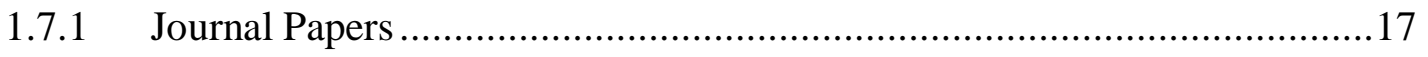

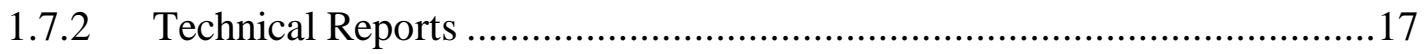

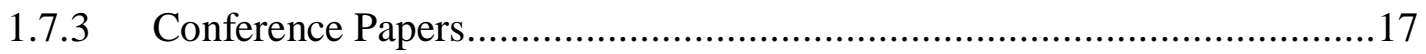

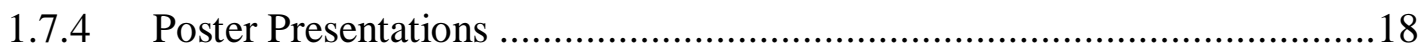

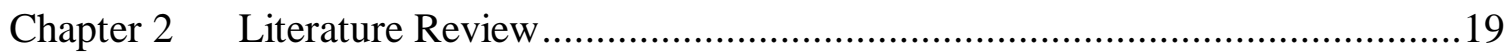

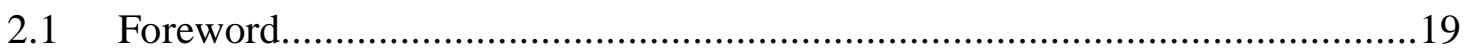

2.2 Diffuser Augmented Wind Turbines.........................................................22

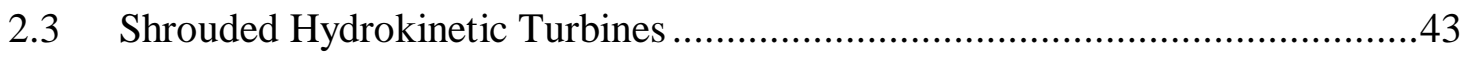

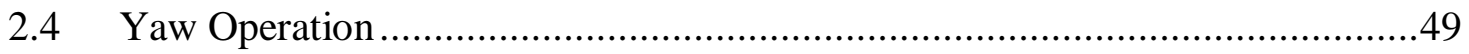

Chapter $3 \quad$ Theoretical Analysis...........................................................................54

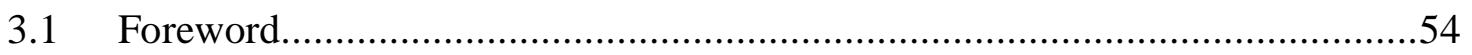

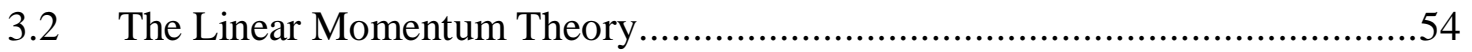

3.3 The Momentum Theory in Yaw Operations ...................................................60 
3.4 The van Bussel Momentum Theory for Shrouded Turbines ...........................63

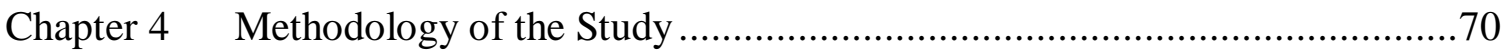

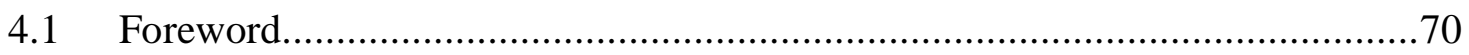

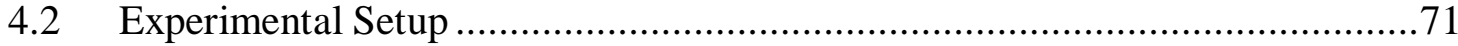

4.2.1 Water Tunnel ......................................................................... 71

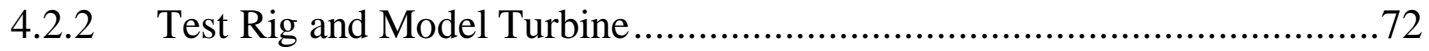

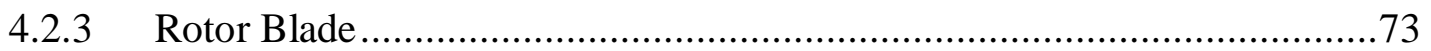

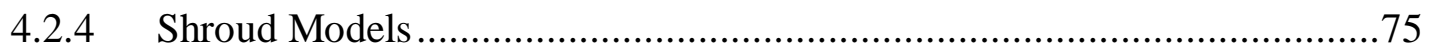

4.3 Background of Diffuser Design ......................................................... 77

4.4 Selection of Area Ratios and Half Angles.......................................... 78

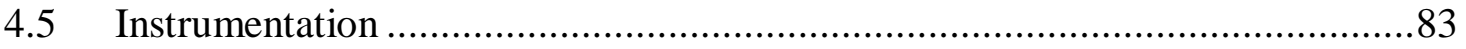

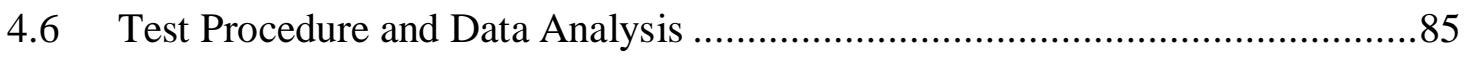

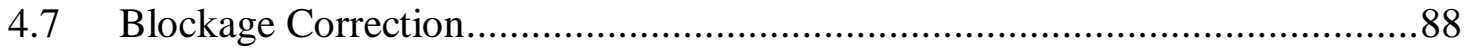

4.8 Uncertainty of Measurements ..........................................................92

Chapter 5 Performance in Inline Operation....................................................95

$5.1 \quad$ Foreword

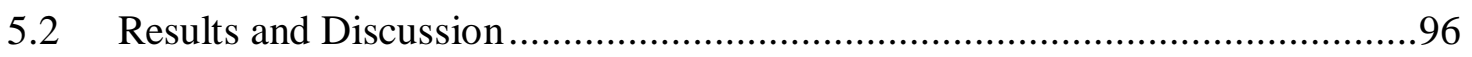

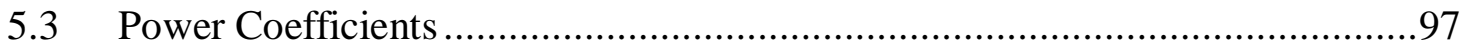

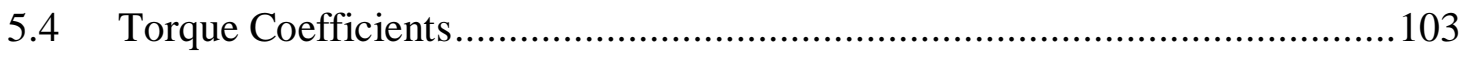

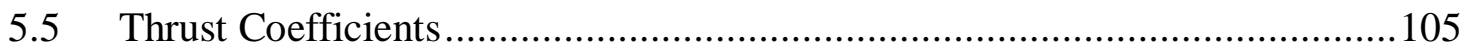

5.6 Relative Increase in Power Coefficients .................................................. 108

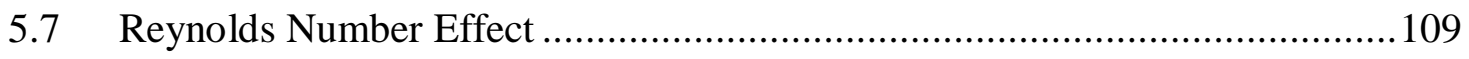

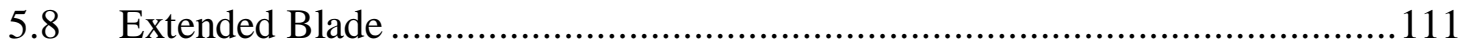


5.9 Shroud or Extended Blade .............................................................. 114

Chapter 6 Performance in Yaw Operation ..................................................... 117

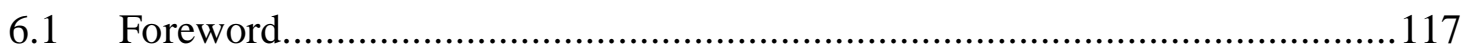

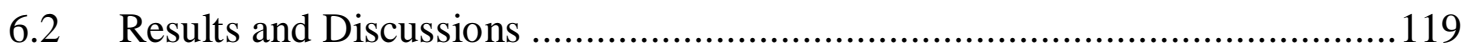

6.3 Power Coefficients .......................................................................... 120

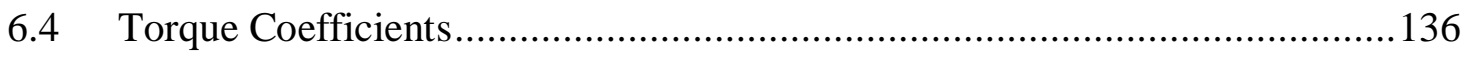

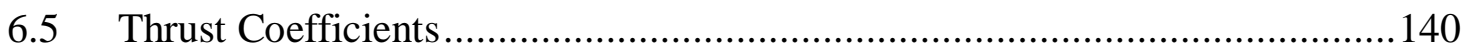

6.6 Comparison to the Linear Momentum Theory ........................................... 144

6.6.1 Wind Turbines Experimental Data ................................................ 144

6.6.2 The Unshrouded Model Turbine....................................................... 146

6.6.3 The Model Turbine with the Shroud ................................................... 148

6.6.4 The Model Turbine with the Diffuser .............................................. 150

6.7 Verification of the Cosine Rules for Shrouded Turbines ........................... 152

6.8 Velocity at the Rotor Plane ................................................................ 154

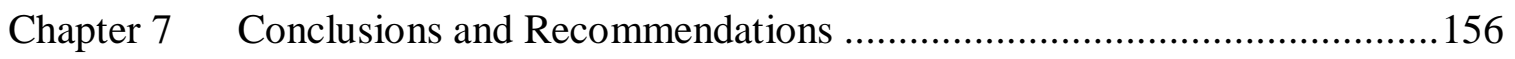

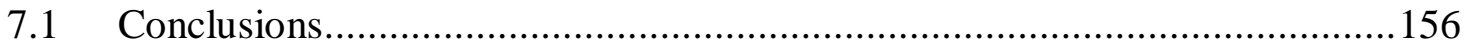

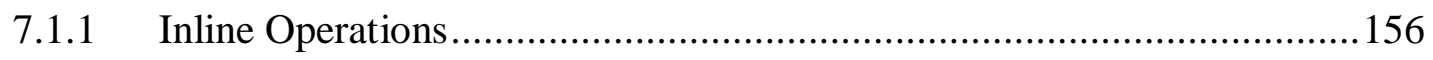

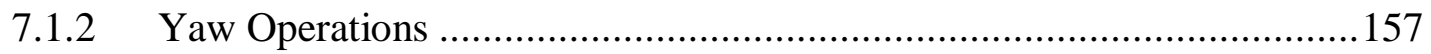

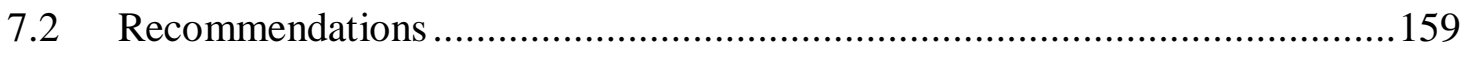

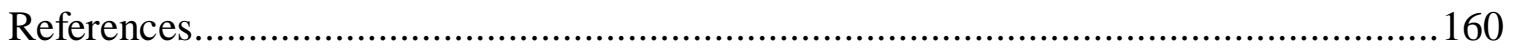

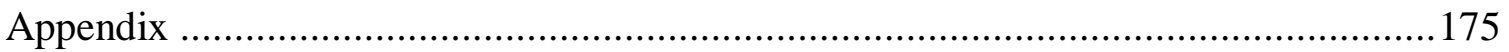




\section{List of Tables}

Table 1.1: Characteristics of the principal wind turbine types ..................................

Table 2.1: Summary of studies done on diffuser augmented wind turbines ..................41

Table 4.1: Physical characteristics of a rotor blade at selected spanwise locations..........75

Table 4.2: Dimensions of the shroud and the diffuser .............................................76

Table 6.1: Maximum power coefficients for the model turbine at $0.7 \mathrm{~m} / \mathrm{s}$ water velocity

Table 6.2: Maximum power coefficients for the model turbine at $0.9 \mathrm{~m} / \mathrm{s}$ water velocity

Table 6.3: Maximum power coefficients for the model turbine at $1.1 \mathrm{~m} / \mathrm{s}$ water velocity

Table 6.4: The cosine rules for the performance reduction in yaw operations and the flow

velocity at the rotor plane 


\section{List of Figures}

Figure 1.1: Lift and drag forces on the root and tip sections of a turbine blade. The blade is twisted from tip to root to turn out of the plane of rotation. .5

Figure 1.2: Performance curves for the most common lift and drag type, horizontal and

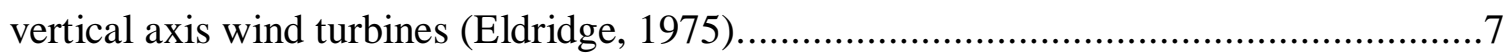

Figure 1.3: Augmentation factors of diffuser augmented wind turbines based on the throat diameter of the diffusers. 14

Figure 1.4: Augmentation factors of diffuser augmented wind turbines based on the exit diameter of the diffusers. .15

Figure 3.1: Control volume over the actuator disk in analysing a wind turbine. Flow velocity undergoes a continuous decrease while pressure drops suddenly across the disc.

Figure 3.2: Deflected wake of a yawed turbine and the induced velocities. The induced velocity at the wake is twice the induced velocity at the disk. .61 Figure 4.1: Front view of the vertical flow loop recirculating water tunnel and its components. .74

Figure 4.2: Front view (left) and side view (right) of the test rig and the model turbine without a shroud installed. The rotor blade is $19.8 \mathrm{~cm}$ in diameter. .74

Figure 4.3: Schematic profile and picture of the shroud (left) and the diffuser (right).....76 Figure 4.4: Tip vortices are generated by the fluid rolling from the pressure side to the suction side of the blades at the tip. .80 
Figure 4.5: Schematic of the test rig and load cell secured between the baseplate and the clamped flat bar (left). The rollers and thoroughly lubricated surfaces allow for a smooth slide of the rig on the tunnel upper rim (right).

Figure 4.6: The model turbine with the shroud (left) and top view of the turbine with the diffuser (right) in the water tunnel during an experiment.

Figure 4.7: The model turbine operating at $10^{\circ}$ yaw angle in a yaw misalignment experiment. The $25^{\circ}$ angle sign was marked later and does not appear in the photo .87

Figure 5.1: Performance coefficient curves of the model turbine at $0.7 \mathrm{~m} / \mathrm{s}$ water velocity. 100

Figure 5.2: Performance coefficient curves of the model turbine at $0.9 \mathrm{~m} / \mathrm{s}$ water velocity. 100

Figure 5.3: Performance coefficient curves of the model turbine at $1.1 \mathrm{~m} / \mathrm{s}$ water velocity. 101

Figure 5.4: A flange shaped diffuser with strong vortices behind the radial brim. 102

Figure 5.5: Torque coefficient curves of the model turbine at $0.7 \mathrm{~m} / \mathrm{s}$ water velocity. .. 103 Figure 5.6: Torque coefficient curves of the model turbine at $0.9 \mathrm{~m} / \mathrm{s}$ water velocity. .. 104 Figure 5.7: Torque coefficient curves of the model turbine at $1.1 \mathrm{~m} / \mathrm{s}$ water velocity. .. 104 Figure 5.8: Thrust coefficient curves of the model turbine at $0.7 \mathrm{~m} / \mathrm{s}$ water velocity. ... 106 Figure 5.9: Thrust coefficient curves of the model turbine at $0.9 \mathrm{~m} / \mathrm{s}$ water velocity. ... 107 Figure 5.10: Thrust coefficient curves of the model turbine at $1.1 \mathrm{~m} / \mathrm{s}$ water velocity. . 107 Figure 5.11: Relative increase in the maximum power coefficients of the shrouded model turbines compared to the unshrouded turbine. 
Figure 5.12: The maximum power coefficients of the shrouded and unshrouded model turbines increase with the Reynolds number at a decreasing rate.

Figure 5.13: The maximum power coefficients of the model turbine with and without the diffuser calculated based on the blade swept area and the diffuser exit area. .113

Figure 5.14: Diameter of an extended blade for generating the same power of the model turbine with the diffuser. .114

Figure 5.15: Comparison of the volumes for the turbine with the diffuser and the turbine with an extended blade generating the same power.

Figure 6.1: Power coefficients of the model turbine in yaw operations at $0.7 \mathrm{~m} / \mathrm{s}$ water velocity.

Figure 6.2: Power coefficients of the model turbine in yaw operations at $0.9 \mathrm{~m} / \mathrm{s}$ water velocity.

Figure 6.3: Power coefficients of the model turbine in yaw operations at $1.1 \mathrm{~m} / \mathrm{s}$ water velocity. 125

Figure 6.4: Relative decrease in the maximum performance coefficients of the shrouded and unshrouded model turbines at $25^{\circ}$ yaw angle compared to zero yaw angle. 129

Figure 6.5: Performance curves of the unshrouded model turbine in yaw operations.... 131 Figure 6.6: Performance curves of the turbine with the shroud in yaw operations. .134 Figure 6.7: Performance curves of the turbine with the diffuser in yaw operations....... 135 Figure 6.8: Torque coefficients of the model turbine in yaw operations at $0.7 \mathrm{~m} / \mathrm{s}$ water velocity. 137

Figure 6.9: Torque coefficients of the model turbine in yaw operations at $0.9 \mathrm{~m} / \mathrm{s}$ water velocity. 138 
Figure 6.10: Torque coefficients of the model turbine in yaw operations at $1.1 \mathrm{~m} / \mathrm{s}$ water velocity.

Figure 6.11: Thrust coefficients of the model turbine in yaw operations at $0.7 \mathrm{~m} / \mathrm{s}$ water velocity.

Figure 6.12: Thrust coefficients of the model turbine in yaw operations at $0.9 \mathrm{~m} / \mathrm{s}$ water velocity.

Figure 6.13: Thrust coefficients of the model turbine in yaw operations at $1.1 \mathrm{~m} / \mathrm{s}$ water velocity.

Figure 6.14: Comparison between the experimental power reduction of wind turbines in yaw angles and the cosine cubed rule of the linear momentum theory.

Figure 6.15: Comparison between the experimental reduction in the thrust at the maximum power of wind turbines in yaw angles and the cosine squared rule of the linear momentum theory. 146

Figure 6.16: Reduction in the maximum power coefficients of the unshrouded model turbine at yaw angles.

Figure 6.17: Reduction in the thrust coefficients at the maximum power of the unshrouded model turbine at yaw angles.

Figure 6.18: Reduction in the maximum power coefficients of the model turbine with the shroud at yaw angles.

Figure 6.19: Reduction in the thrust coefficients at the maximum power of the model turbine with the shroud at yaw angles. 150 Figure 6.20: Reduction in the maximum power coefficients of the model turbine with the diffuser at yaw angles. 151 
Figure 6.21: Reduction in the thrust coefficients at the maximum power of the model turbine with the diffuser at yaw angles. 152

Figure 6.22: Comparison between the experimental power reduction of a diffuser augmented wind turbine and the rule of cosine of the yaw angle deduced in this study. 


\section{Chapter 1 Introduction}

\subsection{Foreword}

Harnessing natural energy resources has been part of all civilizations in history. Man has always utilized the available energy in nature including wind, water, solar, fossil fuels, etc. to facilitate his life. Since ancient times wind energy was used to power simple machines (Riegler, 1983). The first historically accepted utilization of a windmill is reported from the tenth century in Persia, presently located on the border of Iran and Afghanistan. It was a vertical axis wind turbine used to convert the kinetic energy of wind to mechanical power for grinding and irrigation (Shepherd, 1990). Although man started with renewable energy such as wind energy, fossil fuels due to their advantages took over in the beginning of the twentieth century (Riegler, 1983). The oil crises in the late 70's and the need for an alternative source of energy brought new attention to renewable energy resources like wind. Different types of turbines including lift and drag types, vertical and horizontal axis turbines were designed and tested (Eriksson et al., 2008). Attempts were also made to the enhancement of wind turbine power generation. Different techniques such as tornado system, tip-vane, vortex-augmenter, and shroud were proposed and tested among which diffuser augmented wind turbines gained popularity (de Vries, 1979). Today, horizontal axis wind turbines dominate the wind turbine technology. Also, in spite of decades of research on diffuser augmented wind 
turbines, modern commercial scale wind turbines are unshrouded and the low density of wind energy is compensated for by increasing the size of blades.

The global energy market shows interest in utilizing a portfolio of renewable energy resources. The growing worldwide energy demand, finite resources of fossil fuels, and regulations on controlling greenhouse gases are a few reasons for such an interest (Haans, 2011). Hydrokinetic energy of rivers and tides is a renewable source of energy which can meet part of the global electricity demand. Horizontal axis hydrokinetic turbines can be used in a similar manner to wind turbines to extract the kinetic energy of water currents. The more predictable behavior of water currents from rivers and tides originated from gravitational forces leads to a more reliable and predictable power generation than wind caused by atmospheric changes (Birjandi et al., 2013). A conventional hydroelectric turbine converts the potential energy of water in the reservoir of a dam to electricity. A hydrokinetic turbine in contrast, utilizes the kinetic energy of water in rivers or ocean streams to generate electricity without making significant changes to the environment (O Rourke et al., 2010). Single applications of hydrokinetic turbines for water pumping or electricity generation go back to the 20th century. Effective research on the technology however wasn't started until the beginning of the 21st century (Fraenkel, 2010). Like for wind turbines, shrouded hydrokinetic turbines have also been investigated as a means of compensating for the relatively low power density of water currents (Khan et al., 2009).

Canada is indeed rich in water resources and has communities that have hydrokinetic energy available nearby for electricity generation at the distributed scale. Canada's 
marine renewable energy sector can become highly competitive in the global energy market serving domestic and global power needs. The vision foresighted in Canada's Marine Renewable Energy Technology Roadmap is to make Canada a global leader in the delivery of river current energy production systems by 2020 (Taylor, 2011). The total extractable mean potential power from in-stream tidal, river current, and wave resources of Canada is about $35,700 \mathrm{MW}$ which is $27 \%$ of the Canadian electricity demand in 2010. A key element in making the marine renewable energy technology vision a reality is to build power generating capacity of $75 \mathrm{MW}$ by 2016, $250 \mathrm{MW}$ by 2020 , and 2,000 MW by 2030 . This would also provide $\$ 2$ billion annual economic value to Canada by 2030 (Taylor, 2011). Horizontal axis hydrokinetic turbines are expected to provide a large portion of the anticipated power generating capacity (Khan et al., 2009). Distributed electricity generation using hydrokinetic turbines can provide enough power for off-grid communities, while a turbine farm can generate larger amounts of electricity. A river or ocean current hydrokinetic turbine is designed to generate electricity in the order of $5 \mathrm{~kW}$ to $1 \mathrm{MW}$ at the rated velocity. The conventional hydroelectric power plants generate electricity in the order of 100 to $1,000 \mathrm{MW}$ at the rated volumetric flow.

\subsection{Hydrokinetic Energy and Horizontal Axis Turbines}

A horizontal axis hydrokinetic turbine is similar in configuration and operation to a wind turbine in which the rotor blade is secured to a horizontal shaft. The output power is a function of the water density, $\rho$, the rotor blade swept area, A, and the water velocity, $\mathrm{V}$, as follows: 


$$
P=\frac{1}{2} C_{P} \rho A V^{3}
$$

where $C_{P}$ is the performance coefficient of the turbine and is a nonlinear function of tip speed ratio. Tip speed ratio is the ratio of the circumferential velocity of the rotor blade tip to the water velocity, $T S R=R \omega / V$ with $\mathrm{R}$ the blade radius and $\omega$ the turbine angular velocity. Setting $C_{P}$ to 1 in Equation (1.1) yields the maximum available kinetic power in the water current for a disk of area A. Albert Betz, a German aerodynamicist, theoretically showed that regardless of the device used, only $16 / 27$ of this maximum power can be extracted (Betz, 1920). This 59\% is known as the Betz limit and shows the theoretical limit for the power generation of any type of kinetic turbines.

Kinetic turbines are classified as horizontal axis and vertical axis turbines. The driving force of the turbine rotor is the basis for another classification of kinetic turbines where they are categorized into drag and lift type machines. While the blade tip velocity cannot exceed the flow velocity in a drag type turbine, it can exceed the flow velocity in a lift type turbine resulting in tip speed ratios as high as 10. In a lift type machine, the lift force generated on the blades produces torque on the turbine shaft which is then converted to electricity in the generator. Fluid flow over a streamlined body like a wing produces different pressures on either sides of the body. The component of the net force resulting from this pressure difference perpendicular to the direction of the flow velocity is the lift force. The drag force is the component of the net force in the direction of the flow velocity, as shown in Figure 1.1. Any flow separation on the blades results in the reduction of the lift force and consequently the power output of the turbine. The circumferential velocity of the blade and hence the relative velocity at each blade section 
changes from tip to root. Therefore, pitch angle of the sections should also change to ensure optimised angles of attack over the span of blade. In order to have optimised angles of attack and to keep the flow attached for the most frequent operation conditions of the turbine, each blade is twisted spanwise from tip to root to turn the blade out of the plane of rotation (Burton et al., 2001). Angle of attack at each section is the angle the relative velocity makes with the chord line of the section airfoil. Pitch angle is the angle between the section airfoil chord line and the plane of rotation. Twist angle of a blade is the difference between the pitch angles of the tip and root sections. Pitch angle for a horizontal axis wind turbine blade is usually chosen so that each spanwise blade section operates at the maximum lift to drag ratio. This results in the turbine delivering the maximum power at the design tip speed ratio. Any variation in the wind speed results in a variation of tip speed ratio when the turbine rotor spins at a constant angular velocity. Therefore, a preferred approach is to account for the wind variation in a particular site over a considerable period of time and choose a spanwise pitch distribution to ensure the maximum power output (Fletcher, 1981).

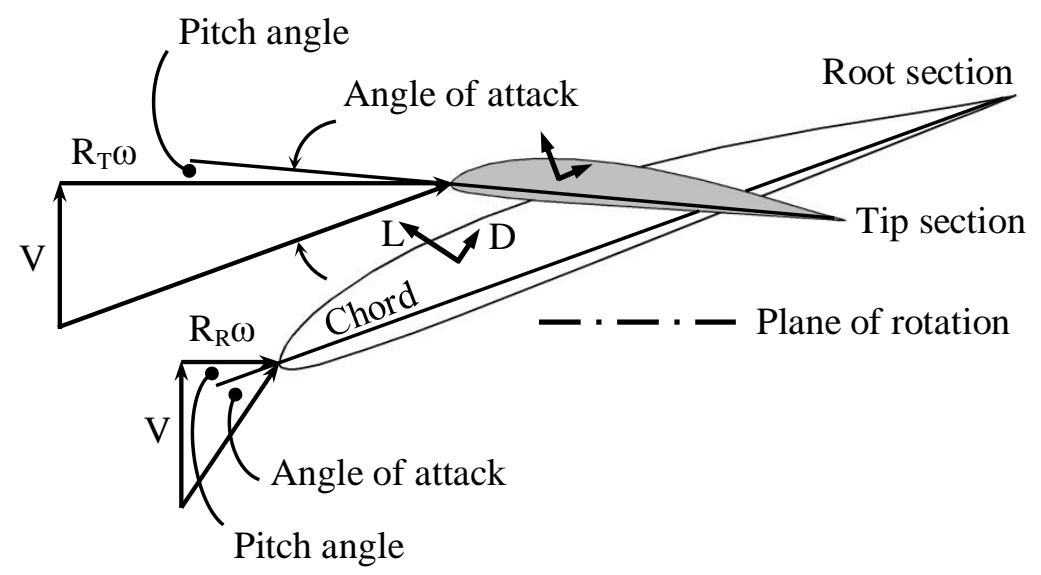

Figure 1.1: Lift and drag forces on the root and tip sections of a turbine blade. The blade is twisted from tip to root to turn out of the plane of rotation. 
Performance coefficient of a hydrokinetic turbine as a function of tip speed ratio is known as the power curve or the performance curve of the turbine. A hydrokinetic turbine has a dome type power curve, the shape of which varies for different turbine topologies and blade arrangements. Figure 1.2 shows the performance curves for different wind turbine types (Eldridge, 1975). Darrieus and Savonius turbines are vertical axis turbines whereas the American windmill, Dutch windmill, and the modern wind turbine are horizontal axis turbines. A turbine with a sharp performance curve experiences power drop when the turbine operates slightly off the narrow range of the optimum tip speed ratio. A flat performance curve indicates that the turbine provides the maximum power over a wider range of tip speed ratio. An ideal turbine would maintain the maximum power for nearly all tip speed ratios and would eventually reach the Betz limit. Power converter electronics ensure the optimum operation in a real turbine. This is done by varying the imposed load on the turbine to control the rotor rpm and achieve the optimum tip speed ratio. Characteristics of the principal wind turbine types are summarized in Table 1.1 (Wortman, 1983). For modern high speed electricity generating turbines the lowest possible torque is desirable in order to reduce the gearbox costs. For water pumping applications a high torque is required to start a positive displacement pump. American multi-bladed windmills developed in the 19th century used for water pumping are slow rotating machines with high starting torque (Burton et al., 2001). 


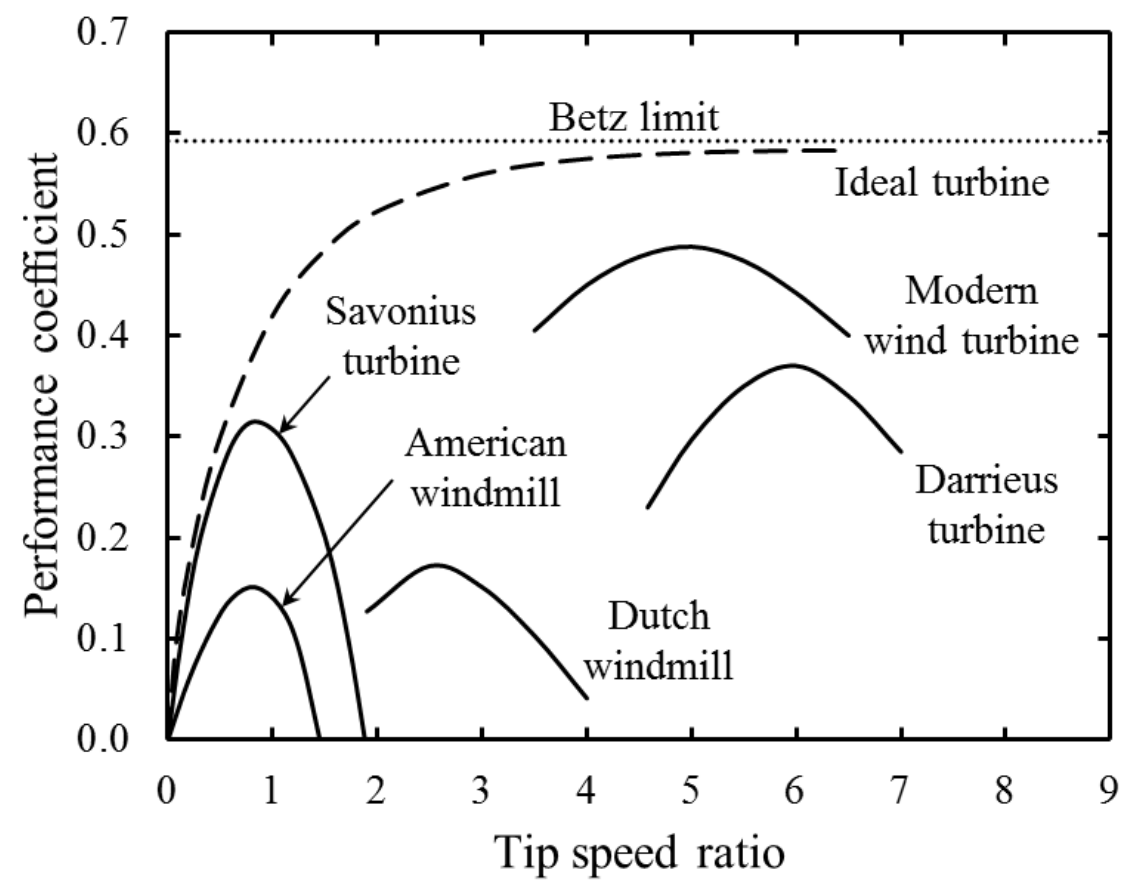

Figure 1.2: Performance curves for the most common lift and drag type, horizontal and vertical axis wind turbines (Eldridge, 1975).

Table 1.1: Characteristics of the principal wind turbine types

\begin{tabular}{cccc}
\hline Machine & Type & Power coefficient & Starting torque \\
\hline Modern wind turbine & Lift & $0.4-0.5$ & Relatively poor \\
Darrieus turbine & Lift & & Poor \\
Savonius turbine & Drag & $0.15-0.3$ & \\
Dutch windmill & Lift & $0.15-0.2$ & Excellent \\
American windmill & Lift & & \\
\hline
\end{tabular}

\subsection{Hydrokinetic Turbines vs. Wind Turbines}

Although a lot can be understood and transferred from wind turbine to hydrokinetic turbine technology, there exist major differences. A hydrokinetic turbine is not simply a 
wind turbine with up to 1000 times higher density working fluid as there are particular aspects regarding the marine environment that need investigations. Bedard (2008) identifies and prioritizes the research, development, deployment and demonstration needs of hydrokinetic energy industry. Experimental testing, environmental issues, and standards are recognized as the top three priorities in the development of hydrokinetic energy industry. A number of topics including better understanding of the effect of diffuser in water current applications, wave kinematics, and turbulence models for sea currents, tidal flows, and rivers are also realized as areas that require further investigation. Inertia loading from decelerating inflow, added mass, damping effect, and buoyancy are the forces not present for a wind turbine that require more investigation. The wake blockage caused by the seabed or the free surface, cavitation, and the mooring system dynamics are also areas that need more research.

Wind turbines run in atmospheric boundary layer and therefore are in the region of reduced wind velocity. However, depending on the deployment depth, it is possible for hydrokinetic turbines to operate out of the boundary layer of water currents. Since $75 \%$ of the kinetic energy of water streams is in the upper half of the water column it is beneficial to deploy a hydrokinetic turbine closer to the water surface (Fraenkel, 2010). It is generally desirable to maximize turbine diameter in order to maximize its power generating capacity. While the manufacturing technology practically controls the maximum diameter of a wind turbine blade, the maximum diameter of a hydrokinetic turbine is determined by the water depth (Ketchman, 2010). A hydrokinetic turbine benefits from blockage effect when the rotor runs close to the flow boundaries; the riverbed or the free surface. This is the same phenomenon happens for wind tunnel tests 
of a wind turbine where the turbine performance is augmented because of the wall blockage effect (Fraenkel, 2010). Other particular aspects of hydrokinetic turbines are the static pressure variations a blade experiences as well as the high axial loads on the rotor blade. The tip of a typical $15 \mathrm{~m}$ diameter rotor blade experiences a $150 \mathrm{kPa}$ cyclic pressure variation as it rotates from its highest to the lowest position. The rotor of a megawatt hydrokinetic turbine experiences thrust loads as large as 1000 to $3000 \mathrm{kN}$ depending on the flow velocity and rotor size (Fraenkel, 2010). Any turbine operating in a harsh river or tidal environment needs to be designed sufficiently robust and reliable to function maintenance free for long periods of time.

\subsection{Shrouds for Hydrokinetic Turbines}

Although the renewable nature of hydrokinetic energy is attractive, some drawbacks of the technology make it currently economically challenging. Low power density of the energy, technical difficulties due to the need for submersible gearbox and generator, and potential impacts on the marine life are some disadvantages of hydrokinetic turbine technology. The low power density and the difficulty to access the turbine remain important challenges this technology faces for commercialization. While a turbine can theoretically extract $59 \%$ of the kinetic power available for the rotor area in a water stream, a performance coefficient of near 0.50 is a practical ideal value. Many different concepts have been proposed to augment the power of a wind turbine among which the shrouded turbine could provide improved capabilities (de Vries, 1979). The concept of diffuser augmented wind turbines has been investigated for more than five decades with no commercial product. Increasing rotor blade diameter is the practical approach to 
reduce the cost of wind energy. Together with the rising efforts to take advantage of hydrokinetic power of rivers and tides, the idea of using shrouded turbines is becoming prevalent. It is assumed that due to the beneficial aspects of water currents compared to wind, a shroud has the potential to benefit the hydrokinetic turbine industry. The velocity and direction of water streams are practically fixed and seasonal changes are more predictable than changes in the wind. This eliminates the need for a yawing mechanism and reduces the extreme loads on the shroud in the storm conditions. A shroud can also contribute to the support structure of a turbine or as a casing for the components of generator (Shahsavarifard et al., 2015).

Some advantages known for a shroud have historically made it an attractive subject for researchers in academia. A shroud increases the turbine power output by increasing the flow velocity and hence the mass flow rate through the rotor blade. Increase in the flow velocity by means of a shroud reduces the cut-in speed of a turbine. A shroud also reduces the blade tip loss known for its negative effect on the turbine performance (Lilley and Rainbird, 1956). Power of a shrouded turbine is less sensitive to changes in the flow direction. A shroud provides gust damping and lowers the fluctuating loads on the blades resulting in lower fatigue and cost. The increased rotational velocity of the rotor in a shrouded turbine reduces the gear ratio and hence the costs of drivetrain (de Vries, 1979).

The increased performance of a shrouded turbine, traditionally stated as the main advantage of a shroud, is obtained by calculating power coefficients based on the rotor diameter and not the shroud exit diameter. It should be noticed that the area used in Equation (1.1) is the maximum area of the device and not the rotor area. Moreover, the 
increased power of a shrouded turbine needs to be weighed against the cost increase due to the added structural elements.

\subsection{Environmental Considerations of Hydrokinetic Turbines}

Hydrokinetic turbine technology faces some environmental challenges such as the impact of the environment on manufacturing, installation, and operation of the turbines. Accumulation of ice, effect of temperature change, and collision of any external object like logs need to be considered in the turbine design. The effect of temperature change on the length of the blades is more serious for a shrouded turbine with a small clearance between the rotating blades and the shroud. There are some additional concerns about possible impacts of a hydrokinetic turbine on the surrounding environment. Bedard (2008) recommended experimental deployment of small to medium turbine arrays to monitor the installation, operation, and decommissioning of hydrokinetic turbines. The collected physical data allows modelling the impacts of the turbines on the marine life and help in finding solutions for any potential environmental issues.

Most of the information on how aquatic animals interact with hydrokinetic turbines comes from the studies on conventional hydropower turbines in which water flows at high speeds through the rotor blade. Hydrokinetic turbines however, function fundamentally different from conventional hydropower turbines and hence affect marine creatures differently (Viehman and Zydlewski, 2014). Local shear stress and pressure change caused by the rotation of blades are much lower for a hydrokinetic turbine than a conventional hydropower turbine (Amaral et al., 2011). The resultant force therefore 
does not affect fish and mammals, however it could injure aquatic organisms (Schweizer et al., 2012). Busch et al. (2013) in a study on two OpenHydro hydrokinetic turbines found a shroud could eliminate the risk of rapid velocity and pressure changes on the marine life. Rotation of an unshrouded turbine blade close to the seabed increases turbidity of water. This increases the risk of collision for animals that use vision for navigation (Wilson et al., 2007) and also affects the habitat of fish and aquatic creatures disrupting their spawning for instance (Cada et al., 2007). Souder et al. (2010) reviewed the studies on the interaction of marine life and hydrokinetic turbines. Potential collision of fish, aquatic reptiles, mammals, and diving birds is considered to be the major environmental concern. The risk of strike is higher close to the tip of the uncovered turbine blades where the velocity is higher. There are however other researchers who believe fish avoid turbines in the same way they avoid any other obstacle like a boat particularly when the obstacle is noisy as in the case of a hydrokinetic turbine (de Robertis and Wilson, 2006; Vabø et al., 2002). Another concern is that a farm of unshrouded turbines could cause large areas of sediment scour or deposition which can alter hydrological regime of the underwater environment (Cada et al., 2007).

Many of the environmental challenges could potentially be resolved by employing shrouded turbines. Risk of collision can be reduced or eliminated by covering the rotating blades with a shroud. Also, the harm caused by lubricant and oil on aquatic plants and animals can be eliminated by designing a gearless shrouded turbine. Examples of such a turbine are reviewed in Section 2.3. A shroud could also eliminate tip vortices responsible for turbine noise and hence reduce the stress factors on aquatic mammals. 


\subsection{Motivation and Objectives of the Study}

After decades of research, wind turbine design has converged to the existing point of technology and power generation from wind has been successfully commercialized. Hydrokinetic turbine technology however, is an emerging technology and is still in the early stages of development. Different designs from lift to drag type, horizontal to vertical axis, and shrouded to unshrouded turbines are proposed and tested in order to find the most suitable design for reliable, affordable, and durable power generation. This study aims to experimentally quantify the effect of a shroud on the performance characteristics of a horizontal axis hydrokinetic turbine in aligned and yawed operations. This will provide a better understanding of turbine operation with and without a shroud in in-line and off-axis flows.

The inconsistent results on the effect of shroud on the performance of kinetic turbines reported in the literature create a vigorous motivation for more research. Further investigation provides an assessment of the subject by critically reviewing previous studies as well as establishing a series of new experiments to address some outstanding issues. As we will see in the comprehensive review of the literature in Chapter 2, diverse results have been reported on the effect of a shroud on the performance of kinetic turbines. Besides the aligned operation, having appropriate understanding of the performance of a shrouded turbine in a yawed flow helps in designing a robust turbine for harsh marine environments. The literature review highlights that there is no conclusive study on the effect of yaw angle on the performance of shrouded hydrokinetic turbines. Figure 1.3 shows the augmentation factors obtained with diffuser augmented 
wind turbines in more than five decades of research. The reported augmentation factors have a broad range from $-36 \%$ to $760 \%$ which shows the divergence among researchers on the effect of shroud. Figure 1.4 is the same augmentation factors recalculated in a proper application of Equation (1.1) based on the diffuser exit diameter. Recalculating the augmentation factors based on the diffuser maximum area corrects for the apparent increase in the performance as the largest cross sectional area of each diffuser model is now considered in the calculations. Although the broad range of the augmentation factors is now narrowed between the range from $-81 \%$ to $88 \%$, it is still large enough to render the studies inconclusive on the effect of shroud. The reasons behind such diverse figures on the effectiveness of a shroud are explained in the next chapter. Figures 1.3 and 1.4 are presented herein for the motivation of this work.

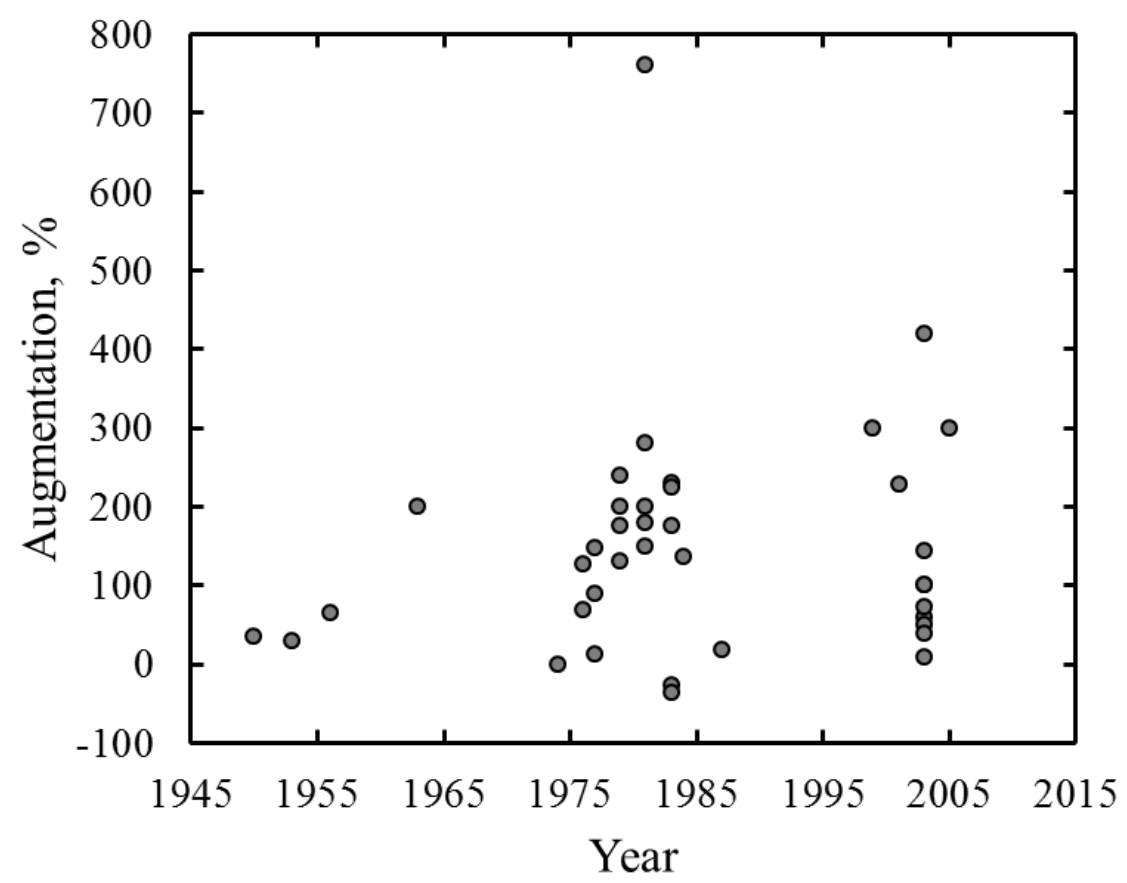

Figure 1.3: Augmentation factors of diffuser augmented wind turbines based on the throat diameter of the diffusers. 


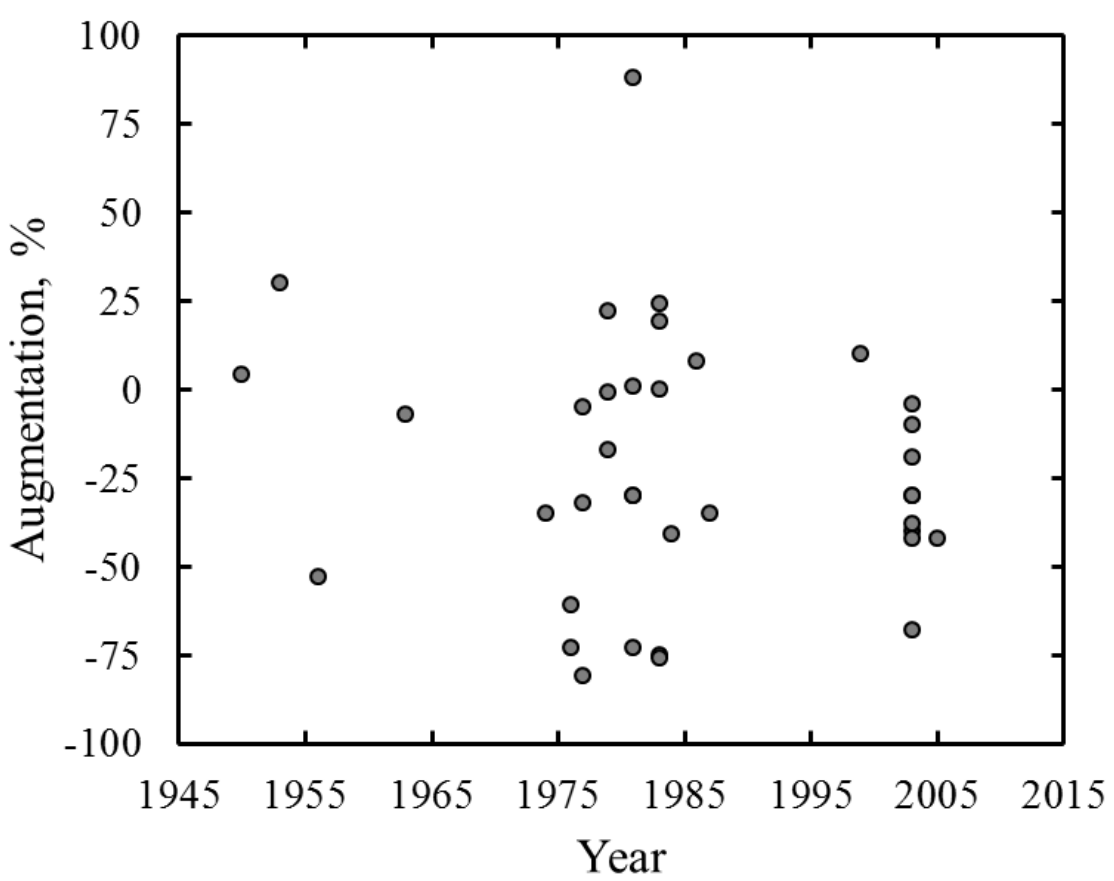

Figure 1.4: Augmentation factors of diffuser augmented wind turbines based on the exit diameter of the diffusers.

Commercialization success of hydrokinetic turbine industry depends on the proper understanding of various areas of the technology. This includes but not limited to research on the turbine types, rotor blade geometry, turbine performance characteristics, deployment methods, and flow characteristics of the deployment site. In this thesis we particularly examine the effect of shrouds on the performance of a horizontal axis hydrokinetic turbine. The geometry of blade is fixed and the performance characteristics of the turbine are investigated for the shrouded and unshrouded configurations. The study includes aligned as well as yawed operations. The primary objective of the study is to experimentally quantify the effect of shrouds on the performance of a horizontal axis hydrokinetic turbine in a laboratory setting. This includes investigating the effect of shrouds on the thrust and output power of the turbine in inline and off-axis flows. In achieving the main objective other important objectives are also fulfilled. The obtained 
results are corrected for free surface proximity and blockage effects. The effect of Reynolds number on the experimental results is assessed and applicability of the results obtained in a laboratory test is evaluated for a full scale model. Influence of shrouds on different operation modes of the turbine is discussed. The results could be used for validation of the on-going and future numerical simulations. The material provided in this thesis will benefit researchers in academia improving understanding of hydrokinetic turbines and the role of apparent efficiency increase, as well as helping industry in making design options.

\subsection{Contributions and Publications}

A new method combining two existing techniques for correcting the free surface proximity and blockage effects is proposed and used for the first time. The effect of shroud on the performance of horizontal axis hydrokinetic turbines is experimentally investigated with reliable and conclusive results. The literature of over fifty years is exhaustively reviewed and the reasons for the divergence among researchers are addressed. Yaw operation of a shrouded turbine is also investigated with the first time conclusive results. New cosine relations for estimating performance loss of a yawed shrouded turbine are deduced from the experimental results and proposed for the first time. The outcomes of this research have been published as journal, conference, and research papers listed below. In addition, the results of numerical simulations of the wakes and eddies due to the flow on the outside of a shroud and its impact on the environment will be published in a journal paper in a near future. 


\subsubsection{Journal Papers}

1 Shahsavarifard, M., Bibeau, E.L., Chatoorgoon, V., 2015. Effect of shroud on the performance of horizontal axis hydrokinetic turbines. Ocean Engineering 96, 215225.

2 Shahsavarifard, M., Bibeau, E.L., 2015. Performance characteristics of shrouded horizontal axis hydrokinetic turbines in yawed conditions. Under review.

3 Shahsavarifard, M., Bibeau, E.L., 2015. A numerical investigation into the effect of shroud on the performance of horizontal axis hydrokinetic turbines. Under review.

4 Shahsavarifard, M., Bibeau, E.L., 2015. Shrouds for hydrokinetic turbines. In preparation.

5 Shahsavarifard, M., Birjandi, A.H., Bibeau, E.L., 2015. Performance characteristics of screw-type hydrokinetic turbines. In preparation.

\subsubsection{Technical Reports}

1 Shahsavarifard, M., Birjandi, A.H., Bibeau, E.L., 2014. Performance Test of Energy Cat 3EC42 Hydrokinetic Turbine. Technical Report CHTTC/TR-002-1014, Canadian Hydrokinetic Turbine Test Center. Winnipeg, Canada.

2 Shahsavarifard, M., Birjandi, A.H., Bibeau, E.L., 2014. Measurements Using a Hydrokinetic Screw-Type Impeller Turbine. Technical Report CHTTC/TR-001-0814, Canadian Hydrokinetic Turbine Test Center. Winnipeg, Canada.

3 Birjandi, A.H., Gaden, D., Shahsavarifard, M., Bibeau, E., 2012. Scaling-up, Hydrodynamic Similarity Laws, and Guidelines to Design Hydrokinetic Turbines for Vertical Hydrokinetic Turbines. Technical Report, Natural Resources Canada. Ottawa, Canada.

\subsubsection{Conference Papers}

1 Shahsavarifard, M., Bibeau, E.L., 2015. Yaw operation of a shrouded horizontal axis hydrokinetic turbine. In: OCEANS '15 MTS/IEEE Genova Conference, Genova, Italy.

2 Shahsavarifard, M., Birjandi, A.H., Bibeau, E.L., Sinclaire, R., 2015. Performance characteristics of the Energy Cat 3EC42 hydrokinetic turbine. In: OCEANS '15 MTS/IEEE Genova Conference, Genova, Italy. 
3 Birjandi, A.H., Shahsavarifard, M., Hamta, A., Bibeau, E.L., Nuefeld, D., 2015. Wake measurement behind a loaded vertical axis hydrokinetic turbine (VAHT) in the field. In: OCEANS '15 MTS/IEEE Genova Conference, Genova, Italy.

4 Shahsavarifard, M., Bibeau, E.L., Birjandi, A.H., 2013. Performance gain of a horizontal axis hydrokinetic turbine using shroud. In: OCEANS '13 MTS/IEEE San Diego Conference, San Diego, USA.

5 Shahsavarifard, M., Bibeau, E.L., 2013. Effect of shroud on the performance of horizontal axis hydrokinetic turbines. In: 4th Annual Marine Renewable Energy Technical Conference, Providence, USA.

\subsubsection{Poster Presentations}

1 Soviak, J., Shahsavarifard, M., Mosallat, F., Bibeau, E.L., 2015. Adaptation of renewable energy generation methods to northern communities. In: Alternative Energy and Food Sovereignty Conference, Winnipeg, Canada.

2 Birjadni, A.H., Woods, J., Shahsavarifard, M., Bibeau, E.L., Molinski, T., 2011. Kinetic turbine research, demonstration and development in Manitoba Rivers. In: Canadian Society for Bio-Engineering (CSBE/SCGAB) Annual General Meeting and Technical Conference, Winnipeg, Canada. 


\section{Chapter 2 Literature Review}

\subsection{Foreword}

Hydrokinetic turbines have been used in single applications for water pumping or electricity generation in the 20th century. However, the effective research on the technology started from the beginning of the 21st century (Fraenkel, 2010). Similar to wind turbine technology, ducted hydrokinetic turbines have also been an attractive subject for researchers in the field (Khan et al., 2009). Literature is rich of decades of research on diffuser augmented wind turbines with theoretical, numerical, and experimental studies on the effect of diffusers on the performance of wind turbines (Phillips, 2003). Two experimental studies in Japan and a theoretical study in the UK were the first works on diffuser augmented wind turbines published in the early 50's. After being silent for a period of time, the oil crisis and the need for an alternative source of energy brought back attention to wind energy in the late 70's and early 80's. Since wind is a low density resource of energy, attempts were made to enhance the power generation of wind turbines using different techniques (de Vries, 1979), among which diffuser augmented wind turbines gained popularity. The effect of diffusers on the performance of wind turbines was sometimes over exaggerated without even paying attention to the practicality of the proposed diffuser designs. Not to mention, this was 
happening in the lack of current technology which allows for ambitious designs of the existing large wind turbine blades. In the first designs, small included angles were chosen resulting in large diffusers having large length to diameter ratios. For the next generations, attempts were made to design compact diffusers by employing boundary layer techniques to increase the efficiency and reduce the length. However, these "compact diffusers" were still large enough to prevent the commercialization of diffuser augmented wind turbines. In the first experimental studies, spinning rotor blades were employed to model a wind turbine in a diffuser (Sanuki, 1950a). In the following years however, many researchers following the recommendation of the theoretical work by Lilley and Rainbird (1956) used perforated disks to create pressure drop across a wind turbine in a diffuser. Using a perforated disk, which is called mesh or gauze screen in the literature, bear a resemblance to the role of an actuator disk in theoretical studies of wind turbines. The screen dissipates energy of the flow and an appropriate choice of permeability determines the desired pressure drop across the turbine rotor blade and hence the thrust force on it. Employing gauze screens prevents the complexity that a spinning rotor blade introduces in the flow and makes pressure measurements across the turbine easier. However, it causes an overestimation in the turbine output power by neglecting the blade profile drag. It also causes an underestimation in the turbine power by ignoring the favorable influence of a spinning rotor blade on diffuser efficiency (Fletcher, 1981). Power output of a diffuser augmented wind turbine is calculated in the literature by multiplying the thrust force on the disk, F, by the flow velocity, V. Performance coefficient is then calculated by dividing this power by the power available in the wind for the area of the disk, A. What is traditionally known in the literature as the 
augmentation factor of a diffuser augmented wind turbine is the ratio of this performance coefficient to the Betz limit:

$$
r=\frac{1}{\frac{16}{27}} \frac{F V}{\frac{1}{2} \rho A V^{3}}
$$

in which $\rho$ is the air density. Different augmentation factors have been reported in the literature by different authors and even sometimes in different publications of one study. The difference in the reported augmentation factors comes from several points. Some researchers use pressure difference across the disk to find the thrust force on the disk, some directly measure the force by strain gauges, and some use the disk loading coefficient as a measure of the thrust force. Some authors use the undisturbed wind velocity as the flow velocity, some measures the velocity at one radial location over the disk and use it as the local flow velocity, and some use an area weighted average velocity over the disk as the flow velocity (Phillips, 2003). Incorrect pressure readings resulted from tunnel blockage in some studies is also accountable for the difference in the reported augmentation factors. For those researchers who used a rotor blade in the experiments, the shaft power is sometimes compared to the Betz limit and sometimes to the power of the unshrouded turbine. Shaft power is the torque measured on the turbine shaft multiplied by its rotational velocity. Due to the above mentioned differences, care should be taken when comparing augmentation factors form different studies. As will be seen in the next section those studies using gauze screens reported high augmentation factors. However, the studies that also used a rotor blade in the same diffusers did not obtain close agreements. 
Studies done on wind and hydrokinetic turbines are reviewed in this chapter. The literature on diffuser augmented wind turbines is reviewed in Section 2.2. Section 2.3 is devoted to hydrokinetic turbines and Section 2.4 addresses the studies on wind turbines in yaw operations. There is no information available on the effect of yaw angle for shrouded hydrokinetic turbines.

\subsection{Diffuser Augmented Wind Turbines}

Research on diffuser augmented wind turbines has been done for more than five decades. As seen in Chapter 1, a variety of results are reported in the literature on the effect of shroud on the performance of a wind turbine. The earliest studies on diffuser augmented wind turbines are the works of Sanuki and Iwasaki from Japan and the study of Lilley and Rainbird in the UK. Sanuki (1950a, 1950b) studied ducted wind turbines as part of an extensive theoretical and experimental study on wind vanes and anemometers. The theoretical study showed a 1.3 times higher flow velocity at the throat of the duct compared to the free stream. He also performed wind tunnel experiments on a small scale turbine with and without a shroud as well as field tests on a full scale shrouded turbine. The turbine had a $300 \mathrm{~mm}$ diameter rotor blade made of curved aluminum sheets and tests were done for $6,4,3,2$, and 1 bladed rotors. The shroud had a cylindrical section at the throat and a conical inlet and exit with an inlet to throat area ratio of 1.58 and an exit to throat area ratio of 1.21. It also had a brim at the exit that increased the exit area ratio to 2.89 . The Reynolds number in the wind tunnel tests was $2 \times 10^{5}$ based on the rotor diameter and wind velocity. The full scale model had a $2 \mathrm{~m}$ diameter rotor blade with Clark-Y airfoil sections and a similar duct as the small scale turbine. The Reynolds 
number in the field tests was $7 \times 10^{5}$ based on the rotor diameter and wind velocity. The power coefficient calculated using the rotor diameter is 0.355 for the three bladed small scale turbine which increased to 0.48 when adding the shroud. This is $4 \%$ increase in the turbine performance when the power coefficient is calculated based on the shroud exit diameter and $53 \%$ decrease when taking the brim diameter into account. The power coefficient of the full scale shrouded turbine obtained in the field tests was 0.43 which is $10 \%$ less than the performance obtained in the wind tunnel. Sanuki concluded that the large conical inlet of the shroud can be regarded to be equivalent to a larger rotor diameter and that the true advantage of a shroud should be examined on this basis. He added the reason for employing a shroud can be its performance in off-axis winds and the protection it provides against storm which he admitted that would be at the cost of a high drag force on the turbine.

Iwasaki (1953) did theoretical and experimental studies on a $1 \mathrm{~m}$ diameter wind turbine with 2, 3, 4, and 6 bladed rotors with a circular ring shroud of $300 \mathrm{~mm}$ length. The Reynolds number was $1-2 \times 10^{5}$ based on the chord length at $r / R=0.75$ and the relative incident velocity. The experimental torque, thrust, and power coefficients were compared to the theoretical values for the shrouded and unshrouded turbines. He is in agreement with the previous results of Sanuki (1950a) that the effect of shroud on the performance of a turbine is due to two factors. First, accelerating the wind through the shroud and second, preventing tip losses caused by the tip vortices with the explanation that the latter has the smaller effect. The experimental results showed a maximum $30 \%$ increase in the performance of the shrouded turbine over the unshrouded turbine which confirms the theoretical results. The theoretical analysis was done for an infinitely long circular 
cylinder as the shroud. In contrast to what Iwasaki obtained, Gaden and Bibeau (2010) and Isensee and Abdul-Razzak (2012) more than five decades later showed that a purely cylindrical shroud degrades the turbine performance.

Lilley and Rainbird (1956) used the one-dimensional momentum theory and vortex theory to study diffuser augmented wind turbines. They theoretically showed the gain in the performance of a ducted wind turbine has two reasons: first, the elimination of blade tip losses and second, increasing the flow velocity through the rotor. Increasing the flow velocity occurs by controlling the flow expansion downstream of the rotor blade. They showed the performance enhancement depends mainly on the internal frictional losses, the shroud exit area ratio, and the external shape of the shroud at exit and therefore the diffuser exit pressure. Inlet contraction ratio of the shroud was found to have less effect on the performance enhancement. Their study showed a shrouded turbine could gain a minimum of $65 \%$ power enhancement compared to the ideal power of an unshrouded wind turbine, the Betz limit, when both turbines have the same rotor diameter. This enhancement was shown to be obtained with a well-designed shroud with an exit area ratio of 3.5. They further stated when the internal losses are neglected the gain in the power of a ducted wind turbine is proportional to the diffuser exit area ratio. They anticipated a diffuser with an expansion ratio of 5 would increase the power enhancement to $85 \%$ however, they questioned whether this gain in the performance could justify the cost of such a large duct. They expected unlikely that the practical performance of a ducted wind turbine exceeds twice the performance of an unshrouded turbine. Lilley and Rainbird recommended experimental studies with gauze screens having various levels of 
perforation to model the rotor blades with different thrust loadings. This recommendation was followed by many researchers in the 80 's.

Kogan and Seginer (1963) performed wind tunnel experiments on a diffuser augmented wind turbine. The wind tunnel had a $60 \mathrm{~cm}$ by $90 \mathrm{~cm}$ test section and the shroud model consisted of a bell shaped intake followed by a short cylindrical section and a diffuser. They used mesh screens to simulate a wind turbine in the shroud. The shroud had an $88 \mathrm{~mm}$ diameter throat and a total length to throat diameter ratio of 6.82 . The diffuser with its $8.5^{\circ}$ included angle and exit to throat area ratio of 3.5 formed more than $85 \%$ of the shroud's length. The small included angle and hence the high length to diameter ratio was chosen based on the conservative design criteria of Robertson (1949) to avoid flow separation in the diffuser. A maximum augmentation factor of 3 was achieved in an inline flow but increased to 3.5 in a $30^{\circ}$ yawed flow. The elevated augmentation factor in the off-axis operation is due to the blockage effect intensified in yaw angles by the large length of the shroud.

Wilson and Lissaman (1974) studied the performance of different concepts of wind power machines. Using the one-dimensional momentum theory and the actuator disc model they compared power coefficients of a ducted and a bare wind turbine. Assuming the exit pressure of the duct equal to the free stream static pressure they found a maximum power coefficient of 0.385 for a shrouded turbine based on the duct exit diameter. This is 35\% less than the performance coefficient of an ideal turbine with a rotor having the same diameter as the duct exit. They concluded the power coefficient of a ducted wind turbine with an exit area ratio larger than 1.54 exceeds the power 
coefficient of an unshrouded turbine when the performance is calculated based on the rotor diameter.

Igra (1981) reported on an experimental study he did a few years before on diffuser augmented wind turbines using a shroud similar to that employed by Kogan and Seginer (1963) with the same throat diameter but a shorter diffuser. The experiments were done in a wind tunnel with a $60 \mathrm{~cm}$ by $90 \mathrm{~cm}$ test section with the Reynolds numbers of $5 \times 10^{4}$ to $3 \times 10^{5}$ based on the shroud diameter. Gauze screens were used to model the turbine and diffuser had a $12.5^{\circ}$ included angle with a length to throat diameter ratio and an exit to throat area ratio of 2. Three stages of ring-shaped flap with NACA 1412 airfoil section were used at the diffuser exit which increased the length to throat diameter ratio to 3.64 and the exit to throat area ratio to 10.3 . The flaps reduced the diffuser exit pressure and hence increased the air speed and mass flow through the shroud. The reduction in the exit pressure was created by directing the accelerated air - in the gap between the flap and the diffuser - into the wake flow. A maximum augmentation factor of 2.8, stated not to be an optimal value, was achieved. This is a low augmentation factor for the high exit area ratio of the shroud. Igra (1976) continued his research on diffuser augmented wind turbines with a shroud having NACA 4412 aerofoil section with an additional circular flap of the same cross section. His turbine was again modeled with mesh screens and the shroud had an $88 \mathrm{~mm}$ diameter throat and a total length to diameter ratio of 3.07. He used a larger wind tunnel with a $3.6 \mathrm{~m}$ by $2.6 \mathrm{~m}$ test section to avoid the $11 \%$ blockage he had in his previous experiments. He tested four variations of the shroud and got a maximum augmentation factor of 2.26 with the turbine having an exit to throat area ratio of 8.33. The maximum augmentation factor was 1.69 for the shroud without the 
circular flap having a length to diameter ratio of 2.27 and an exit area ratio of 4.37 . Having reduced the blockage and wall effects, the performance of the shrouded turbine unlike for Kogan and Seginer (1963) - decreased in yaw operations. The performance reduction was slow for yaw angles up to $10^{\circ}$ but became rapid for larger angles. After performing experiments with gauze screens to simulate turbine rotors, Igra (1977) employed a two-stage turbine. The turbine was composed of a 7-blade stator preceded by an 8-blade rotor. The shroud had a NACA 4412 airfoil cross section with a $39 \mathrm{~cm}$ diameter throat, a length to diameter ratio of 5.26, and an exit area ratio of 6.06. The experiments were done in the same previous wind tunnel with the Reynolds numbers of $3.8 \times 10^{5}$ to $8 \times 10^{5}$ based on the wind velocity and the throat diameter. He got a maximum performance coefficient of 0.67 based on the throat diameter of the shrouded turbine. However, a value of 0.11 is obtained when calculating the performance using the shroud exit diameter. Igra obtained higher power in yaw operations: at a $30^{\circ}$ yaw angle he found the increase to be as high as $30 \%$ more than in the inline configuration. This elevated power was due to the large length to diameter ratio of the shroud which made a higher blockage in yaw operations. He believed the high lift of the shroud due to higher angles of attack in yawed conditions was responsible for the elevated power. After doing wind tunnel tests, Igra (1984) performed field tests on a 3 m rotor diameter shrouded turbine with a length to diameter ratio of 2.67 and an exit area ratio of 4 . In a $5 \mathrm{~m} / \mathrm{s}$ wind speed he got a power coefficient of 1.40 based on the rotor diameter which is 0.35 based on the shroud exit diameter. The higher performance obtained in the field tests can be explained by the effect of Reynolds number and turbulence on the onset flow that reduced the flow separation occurred in his wind tunnel model. 
Lewis et al. (1977) organized theoretical and experimental studies on shrouded wind turbines. They used the one-dimensional momentum theory to design a small scale diffuser and designed a large scale diffuser based on the results of the experiments they did on the small diffuser. They used gauze screens in the experiments with the small diffuser and an eleven bladed rotor with circular arc profile blades with the large diffuser. The throat diameter for the small duct and the large duct was $80 \mathrm{~mm}$ and $500 \mathrm{~mm}$, respectively. The exit area ratio was 2.44 for the small diffuser and 2.56 for the large diffuser and both diffusers had a length to diameter ratio of 1.49. Although they observed separated flow in the small diffuser with $32^{\circ}$ included angle, they designed the large diffuser with the same diffusion angle. They expected the swirl behind the rotor blade together with the high level of turbulence to eliminate flow separation in the large diffuser. They also added an inlet to the duct to help stabilize the entrance flow and improve flow stability in gust cross winds. The large scale turbine was tested in a $1.52 \mathrm{~m}$ diameter wind tunnel at a Reynolds number of $3 \times 10^{5}$ based on the rotor diameter and wind velocity. A maximum power coefficient of 0.52 based on the rotor diameter was achieved with the shrouded turbine while the unshrouded turbine had a maximum power coefficient of 0.21 .

Grumman Aerospace Corporation conducted extensive research on diffuser augmented wind turbines. Their aim was to make a compact diffuser with a small length to diameter ratio and a large exit area ratio to minimize the cost (Oman et al., 1977). They worked on two types of diffusers referred to as "boundary layer control" and "ring wing" diffusers. The boundary layer type was a straight thin wall diffuser whereas the ring wing type had an aerofoil cross section. They did the first phase of their experiments on a set of 150 
diffuser configurations in order to find a baseline diffuser. The experiments were done in a $30 \mathrm{~cm}$ diameter open jet wind tunnel. The set included diffusers with $5 \mathrm{~cm}$ diameter inlet having exit area ratios ranging from 1.28 to 4.94 and included angles of $40^{\circ}, 60^{\circ}$, $80^{\circ}$, and $90^{\circ}$ (Foreman and Gilbert, 1979a; Gilbert and Foreman, 1983, 1979; Gilbert et al., 1978). Five mesh screens with different disc loading coefficients were used to simulate a turbine in the diffusers. They also examined the effect of an inlet ring, auxiliary slots, internal turbulence trip tape, various leading edge shapes and trailing edge configurations on the performance of a diffuser. The outcome of the first phase of their study was the development of their baseline design: a straight wall diffuser with a $60^{\circ}$ included angle. The baseline diffuser had an exit area ratio of 2.78 and a length to diameter ratio of 0.5 and resulted in a maximum augmentation factor of 1.89 . They chose the straight wall diffuser configuration and not the one with aerofoil cross section because of its greater peak augmentation as well as its relatively simple fabrication (Oman et al., 1977). They observed the included angle and exit area ratio control the amount of flow separation in the diffuser. This in turn influences the diffuser exit pressure and consequently the diffuser performance. They observed the flow starts to separate from the diffuser inner wall beyond the exit area ratio of 3. They also found close proximity of a shroud wall to the blades tip prevents tip losses (Gilbert and Foreman, 1983). Grumman Corp. performed the next phase of the research in a $2.1 \mathrm{~m}$ by $3 \mathrm{~m}$ wind tunnel on a diffuser with a 10 times larger inlet but the same exit area ratio and length to diameter ratio as the baseline diffuser. A maximum augmentation factor of 2.3 was achieved with the large diffuser (Foreman and Gilbert, 1979b). The augmentation factor was based on the throat diameter and was $22 \%$ more than the augmentation 
achieved with the small model. This increase in the augmentation factor was due to the higher Reynolds number and tunnel blockage in the latter experiment. Grumman Corp. next decided to replace the gauze screens with rotor blades and investigated rotor/stator turbine configurations to use in the diffuser. They showed the power curve of a rotor has a wider peak and that a rotor is more effective than a rotor/stator configuration. They used $457 \mathrm{~mm}$ diameter, two and three bladed rotors with a NACA 63(420)-422 aerofoil profile. They tested the shrouded turbine with the two bladed rotor in a $1.8 \mathrm{~m}$ by $1.8 \mathrm{~m}$ open jet wind tunnel (Foreman and Gilbert, 1983) and the shrouded turbine with the three bladed rotor in a $2.1 \mathrm{~m}$ by $3 \mathrm{~m}$ closed-loop wind tunnel (Foreman and Gilbert, 1979b). Using the same diffuser with the exit area ratio of 2.78 created respectively $14 \%$ and $7 \%$ tunnel blockage in the two wind tunnels. Augmentation factors of 2.75 and 3.4 based on the rotor diameter were obtained for the two and three bladed turbines, respectively. The reported augmentation factors were calculated by multiplying the thrust force on the rotor measured with a strain gauge by the wind velocity measured at the rotor plane. Phillips (2003) assuming an average rotor efficiency of $24 \%$ estimated a maximum shaft power augmentation of 0.72 for the two bladed turbine. This is a performance coefficient of 0.43 based on the rotor diameter and 0.15 based on the diffuser exit diameter. Grumman Corp. knew from their own experimental results in (Foreman and Gilbert, 1979a) and also from the results of an inviscid flow modelling by Loeffler Jr. (1981) that increasing the diffuser included angle improves its performance. Therefore, in their final step of research on diffuser augmented wind turbines they decided to increase the diffuser included angle. They anticipated a diffuser with an exit area ratio of 4 and an included angle of $80^{\circ}$ or $90^{\circ}$ would attain an augmentation factor up to 6 or 8 . They used the two 
bladed $457 \mathrm{~mm}$ diameter rotor from their previous experiments and designed a diffuser with a $45^{\circ}$ half angle and a length to diameter ratio of 0.488 (Foreman et al., 1983). Three variations of the diffuser with different exit area ratios were examined and a maximum augmentation factor of 3.25 was obtained with a diffuser having an exit area ratio of 2.62. Phillips (2003) in reviewing this result estimated the maximum shaft power augmentation to be 0.64 . This is equal to a performance coefficient of 0.38 based on the rotor diameter and 0.14 based on the diffuser exit diameter. Therefore, the $60^{\circ}$ included angle diffuser was more efficient than the $90^{\circ}$ included angle diffuser.

de Vries (1979) in a theoretical study using the linear momentum theory investigated shrouded wind turbines. The study aimed to provide an insight into the concept of shrouded turbines rather than offering a basis for designing a shroud. An optimum value of about 2 was estimated for the power coefficient of a shrouded wind turbine. The estimation was based on the order of magnitude analysis for an annular wing shroud using the vortex theory as well as considering the results of then available experimental measurements. He stated such a performance can be obtained at the expense of a huge structure around the shrouded wind turbine. He complemented the study by summarizing the advantages and disadvantages of a shrouded wind turbine. Increased energy output due to the increased mass flow, small increase in allowable pressure difference across the turbine, and less blade tip losses were stated as the advantages. Moreover, less sensitivity of the energy output to off-axis flows, lower fluctuations in the blade loads due to the damping effect of the shroud on the variations of wind direction were also listed as the advantages. The higher rpm of turbine shaft and a consequently reduced gear ratio were mentioned as the advantages of a shrouded turbine as well. A disadvantage however was 
said to be the high cost of a huge structure around the turbine which has to be aligned to the wind direction.

Fletcher (1981) in a numerical study used the blade element momentum theory to investigate diffuser augmented wind turbines. He incorporated the influence of a diffuser into the analysis by introducing an exit pressure coefficient and a diffuser efficiency; two empirical values he obtained from the available experimental results. Calculations showed a diffuser with an exit area ratio of 4 enhances the turbine performance by a factor of 2.5 which can increase to 3 with a diffuser having an exit area ratio of 6 . The study showed a diffuser augmented wind turbine has a flatter power curve than an unshrouded turbine. A flat power curve characterises a stable turbine operation where the maximum power occurs at a wider range of tip speed ratio. Calculating the stated augmentation factors based on the diffuser exit area shows a decrease in the power coefficient of more than $30 \%$ compared to an unshrouded turbine.

Loeffler Jr. (1981) in a numerical study using the method of singularities investigated diffuser augmented wind turbines. He studied conical and flanged diffusers with slotted walls as a boundary layer control method. The flanged diffuser was a conical diffuser with a radial brim at the exit. The turbine rotor was modeled as an actuator disk to produce a pressure drop at the inlet of the diffuser. Calculations were done for $60^{\circ}$ and $80^{\circ}$ diffuser included angles. The maximum augmentation for the diffusers with $80^{\circ}$ included angle was more than $30 \%$ higher than the maximum augmentation of the diffusers with $60^{\circ}$ included angle. Also, the flanged diffusers were found to be more efficient than the conical ones. A maximum power coefficient of 5.1 was obtained with a 
flanged diffuser with $80^{\circ}$ included angle having an exit area ratio of 4.58 . The maximum performance coefficient was 3.8 for a conical diffuser with $80^{\circ}$ included angle and an exit area ratio of 3.76. Calculating the performance coefficients based on the diffuser exit area shows an $88 \%$ increase in the performance of the flanged diffuser and a $1 \%$ increase in the performance of the conical diffuser with respect to the Betz limit. Comparing the results to the existing experimental values, the author stated that the study overestimates the turbine performance. The reason is that the inviscid flow simulation is unable to predict the effect of flow separation on the downstream face of the flange.

Riegler (1983) in a theoretical study on wind turbines stated that there exists a direct relationship between the flow velocity reduction and mass flow rate in an unshrouded wind turbine. For a diffuser augmented wind turbine, however, a higher mass flow rate per turbine area can be obtained together with a higher velocity reduction. The paper mainly investigates unshrouded wind turbines but also provides a graph for power coefficient of a diffuser augmented wind turbine. A maximum power coefficient of 1.96 is shown to be attainable with a diffuser having an exit area ratio of 2.78 . This is a $230 \%$ increase in the performance with respect to the Betz limit and based on the rotor diameter which becomes a $19 \%$ increase when the power coefficient is calculated based on the diffuser exit area.

Dick (1986) applied the momentum theory to wind energy concentrator systems and showed the power coefficients based on the frontal area of any concentrator system can be written in a form similar to that of an unshrouded wind turbine. The concentrator systems include shrouds, tip vanes, and tornado systems. A system function was defined 
as a relationship between the wind velocity at the rotor disc and behind the system. Comparing the above mentioned concentrator systems Dick made a general conclusion that only a shroud can make a considerable improvement to the performance of a wind turbine. Applying the model to then available experimental results showed the total concentration coefficient for the most efficient shrouded turbine of the time (Gilbert and Foreman, 1983) is larger than the area ratio of the diffuser by a factor of 1.08. This reveals the energy available in a streamtube with a cross section equal to the diffuser exit area is concentrated on the turbine with some augmentation due to the existence of the shroud. In other words the investigated shrouded turbine generates $8 \%$ more power than an unshrouded turbine with a rotor diameter equal to the diffuser exit diameter. Dick concluded this is a further confirmation to the applicability of the Betz limit to shrouded turbines when the shroud exit area is used to calculate the performance coefficient.

Nagai and Irabu (1987) carried out a theoretical study on diffuser augmented wind turbines using the momentum theory. Utilizing the Bernoulli's and continuity equations along with the actuator disk model, they derived the power coefficient of a turbine as a function of the turbine load factor, diffuser area ratio, and diffuser efficiency. They concluded that with no momentum loss in the diffuser flow, the maximum power coefficient of the turbine based on the rotor area is about 0.7 for the optimum combination of load factors and area ratios. This is 0.385 when the performance coefficient is calculated based on the diffuser exit area.

Phillips et al. (1999) reported on experimental and numerical studies on a diffuser augmented wind turbine. The wind tunnel experiments were done on a shrouded and an 
unshrouded wind turbine using calibrated gauze screens as well as a $457 \mathrm{~mm}$ diameter rotor blade. The diffuser had an exit area ratio of 3.8 and the experiments Reynolds number ranged from $2.1 \times 10^{5}$ to $3.4 \times 10^{5}$ based on the wind velocity and the rotor diameter. The numerical simulations were done in the commercial code PHOENICS on an axi-symmetric model of the diffuser using $\mathrm{k}-\varepsilon$ turbulence model. The turbine was modelled as a flow resistance to apply a pressure drop across the rotor plane analogous to the gauze screens in the wind tunnel experiments. Flow characteristics such as swirl and tip vortices specific to a spinning rotor blade were not incorporated in the simulations. Results of the CFD calculations did not match the experimental power coefficient and base pressure. The $12 \%$ blockage of the turbine in the wind tunnel was assumed to be the reason. The authors then modified the CFD model by adding a solid boundary around the turbine at a distance equal to the hydraulic diameter of the wind tunnel. This improved the base pressure coefficients but resulted in an over-prediction of the power coefficients. The CFD simulations also did not predict the flow separation seen in the wind tunnel experiments. The maximum experimental power coefficient based on the rotor diameter was 1.43 and 0.33 for the shrouded and unshrouded turbines, respectively. Calculating the power coefficient of the shrouded turbine based on the diffuser exit area shows an increase of about $10 \%$ compared to the unshrouded turbine. The power coefficient obtained with gauze screens is $40 \%$ more than the power coefficient obtained with the rotor blade. They also performed off-axis flow experiments and observed yaw angles up to $10^{\circ}$ have little effect on the turbine performance.

Frankovic and Vrsalovic (2001) reported on a patented design of a nozzle augmented wind turbine which is basically a wind turbine with a converging duct at the inlet. 
Dimensions of the turbine and nozzle are not provided in the paper and the nozzle is stated to increase the flow velocity through the turbine rotor by a factor of 2.5 . Based on this velocity increase and the energy output obtained from one prototype, the authors stated that the nozzle augmented wind turbine generates 3.28 times more energy than an unshrouded turbine. This conclusion is based on the results of an experiment on a specific turbine at a specific location in Croatia.

Bet and Grassmann (2003) did numerical simulations using the commercial code Star$\mathrm{CD}$ to model a $56 \mathrm{~cm}$ diameter 36 -bladed rotor wind turbine. They used such a rotor for simplicity of modelling and less computational time required. No details are provided for the rotation and turbulence models used. A maximum performance coefficient of 0.39 was found for the unshrouded turbine at the Reynolds number of $3.7 \times 10^{5}$ based on the rotor diameter and free stream velocity. They then simulated a shrouded turbine using two shroud models; first an annular ring with aerofoil cross section and second a shroud consisted of two successive concentric annular rings. The first shroud having an exit area ratio of 2.2 resulted in a $60 \%$ increase in the turbine performance. The second shroud with an exit area ratio of 3.4 doubled the turbine power coefficient. Calculating the performance coefficients based on the exit area of the shrouds shows respectively a $30 \%$ and $40 \%$ reduction in the performance of the first and second shrouded turbine with respect to the unshrouded turbine. Grassmann et al. (2003a) used the double ring shroud of (Bet and Grassmann, 2003) to experimentally investigate the effect of shroud on the performance of a wind turbine. The turbine had a $56 \mathrm{~cm}$ diameter 5-bladed rotor and a shroud with an $84 \mathrm{~cm}$ diameter outer ring. The experiments were carried out with the shrouded and unshrouded turbines mounted on a truck moving with two different 
constant velocities. The shroud model was observed to increase the turbine performance by $50 \%$ and $100 \%$ at the higher and lower velocities examined, respectively. This reveals the more effectiveness of a shroud in lower wind speeds. Calculating the performance coefficients based on the diffuser exit area shows more than $30 \%$ and $10 \%$ decrease in the performance of the shrouded turbine compared to the unshrouded turbine. Grassmann et al. (2003b) numerically simulated this turbine using a rectangular rotor blade and a new shroud. They considered a shrouded turbine as a partially static turbine in which the non-rotating part of system, the shroud, contributes in energy extraction process. They were able to obtain an augmentation factor of 5.2 by employing a $1.1 \mathrm{~m}$ long annular wing shroud having a highly cambered thin airfoil profile and an exit diameter of $2.6 \mathrm{~m}$. Calculating the power coefficient of the shrouded turbine based on the shroud exit diameter however shows a $4 \%$ reduction in the performance of the shrouded turbine compared to the unshrouded turbine.

Phillips (2003) in conjunction with a New Zealand based company and using the results of the final step of Grumman Corp.'s experiments (Foreman et al., 1983) designed a diffuser augmented wind turbine. The study consisted of numerical simulations and wind tunnel tests as well as field measurements. The full scale turbine used in the field tests had a 7.3 m diameter rotor blade and a diffuser with an overall length to diameter ratio of 0.55 and an exit area ratio of 3.41. The tests resulted in a maximum power coefficient of 0.64 based on the rotor diameter which is 0.19 based on the diffuser exit diameter. The numerical simulations were done on an axi-symmetric model of the shrouded turbine in the commercial finite volume code PHOENICS. The turbine rotor was modeled as a sudden pressure drop across the blade plane. The CFD simulations resulted in a $65 \%$ 
larger augmentation factor of 1.78 compared to the full scale experiments. The overprediction of the performance in the numerical simulations has two reasons. First, simulating the rotor blade as an actuator disk cannot represent the flow behaviour around a rotating rotor blade. Second reason is the inability of k- $\varepsilon$ turbulence model in capturing the flow separation observed in the experiments. The inlet and exit geometry of the diffuser with the boundary layer control slots at the exit were specially designed to improve the performance of the diffuser. However, separated and reversed flow was observed at the inlet and exit of the diffuser in the field experiments. Making modifications to the diffuser in order to overcome the drawbacks of the first design and reduce separation resulted in three new diffuser models. The best of the three new models had an augmentation factor of 1.73 achieved in a $3 \mathrm{~m}$ by $3 \mathrm{~m}$ open jet wind tunnel using gauze screens. This was a multi-slotted diffuser with a $0.48 \mathrm{~m}$ diameter throat, an overall length to diameter ratio of 0.48 and an exit area ratio of 3 . A maximum power coefficient of 0.90 based on the rotor diameter was obtained in the experiments using a rotor blade which is 0.30 based on the diffuser exit diameter. The power coefficient for the unshrouded turbine was measured 0.37 which is larger than the performance coefficient of the shrouded turbine based on the exit diameter. Phillips in the final step of the diffuser design examined a multi-slotted diffuser which was believed to be the most cost effective diffuser augmented wind turbine to the date. This was a diffuser with an overall length to diameter ratio of 0.35 and an exit area ratio of 2.22 . The new diffuser augmented wind turbine attained a maximum power coefficient of 0.82 based on the rotor diameter and 0.37 based on the diffuser exit diameter. The low power coefficients 
and high drag loads of the diffuser augmented wind turbines in this research practically put an end to the efforts on making commercial scale diffuser augmented wind turbines.

Abe et al. (2005) conducted experimental and numerical studies on a small wind turbine with a flanged diffuser. Experiments were conducted in a $2.5 \mathrm{~m}$ by $1.5 \mathrm{~m}$ wind tunnel on a turbine consisted of a $388 \mathrm{~mm}$ diameter three bladed rotor and a shroud with an inlet and a diffuser with a radial brim at the exit. The shroud had an overall length to diameter ratio of 1.59 and an exit area ratio of 6.81 . The CFD analysis was done on a two dimensional model of the turbine utilizing the actuator disc concept to model the turbine rotor. They found the diffuser enhances the turbine performance by a factor of 4 . However, calculating the power coefficient based on the diffuser exit diameter shows a $42 \%$ reduction compared to the unshrouded turbine. Normalizing the power coefficients by the flow mean velocity behind the rotor blade, they found the same maximum performance coefficient for the shrouded and unshrouded turbines. They considered this as a characteristic of the particular diffuser used in their experiments. However the present author explains the reason for this observation in Section 3.4 with the aid of the van Bussel momentum theory. In brief, the theory explains that a diffuser increases the flow rate through the rotor blade whereas the amount of energy extracted per unit of volume is the same for a diffuser augmented wind turbine and an unshrouded turbine. The authors published several other papers on the numerical and experimental studies they did on the flanged diffuser (Abe and Ohya, 2004; Abe et al., 2006; Ohya and Karasudani, 2010; Ohya et al., 2008; Toshimitsu et al., 2008). 
Widnall (2009) following the method of Lewis (1991), developed an incompressible potential flow vortex method to study the flow field in a ducted wind turbine. She used vortex panels to define the shape of a duct and also to account for the flow changes due to pressure drop across the rotor disk. Numerical calculations were done for a shrouded wind turbine with streamlined ducts having exit area ratios of 2,3 , and 4 . The results indicated the power coefficients calculated using the shroud exit diameter show no augmentation compared to the Betz limit. Widnall concluded this as a numerical verification for the van Bussel (2007) momentum theory.

Table 2.1 summarizes the diffuser augmentation factors obtained in the studies reviewed in this section. The augmentation factors are recalculated based on the diffuser exit diameter and are shown based on the throat as well as the exit diameter. A positive augmentation factor shows the percentage increase and a negative value shows the percentage decrease in the performance of the shrouded turbine. The last column in the table shows whether the augmentation factor is stated with respect to the Betz limit or is compared to the performance of the unshrouded turbine. For the studies reporting multiple augmentation factors for a diffuser, the highest augmentation factor is listed in the table. 
Table 2.1: Summary of studies done on diffuser augmented wind turbines

\begin{tabular}{|c|c|c|c|c|c|c|c|}
\hline \multirow[b]{3}{*}{ Author(s) } & \multirow[b]{3}{*}{ Year } & \multirow[b]{3}{*}{$\begin{array}{c}\text { Type of } \\
\text { study }\end{array}$} & \multirow[b]{3}{*}{$\begin{array}{c}\text { Turbine } \\
\text { model }\end{array}$} & \multirow{2}{*}{\multicolumn{4}{|c|}{$\frac{\% \text { Augmentation }}{\text { Based on }}$}} \\
\hline & & & & & & & \\
\hline & & & & $\begin{array}{c}\text { Exit area } \\
\text { ratio }\end{array}$ & $\begin{array}{c}\text { Throat } \\
\text { diameter }\end{array}$ & $\begin{array}{c}\text { Exit } \\
\text { diameter }\end{array}$ & $\begin{array}{c}\text { Compared } \\
\text { to }\end{array}$ \\
\hline Sanuki & 1950 & $\mathrm{E}$ & $\mathrm{RB}$ & 1.29 & 35 & 4 & UT \\
\hline Iwasaki & 1953 & $\mathrm{~T}, \mathrm{E}$ & $\mathrm{RB}$ & 1 & 30 & 30 & UT \\
\hline Lilley \& Rainbird & 1956 & $\mathrm{~T}$ & $\mathrm{AD}$ & 3.5 & 65 & -53 & $\mathrm{BL}$ \\
\hline Kogan \& Seginer & 1963 & $\mathrm{E}$ & MS & 3.5 & 200 & -7 & $\mathrm{BL}$ \\
\hline Wilson \& Lissaman & 1974 & $\mathrm{~T}$ & $\mathrm{AD}$ & 1.54 & 0 & -35 & BL \\
\hline Igra & 1976 & $\mathrm{E}$ & MS & 8.33 & 126 & -73 & $\mathrm{BL}$ \\
\hline Igra & 1976 & $\mathrm{E}$ & MS & 4.37 & 69 & -61 & BL \\
\hline Igra & 1977 & $\mathrm{E}$ & $\mathrm{RB}$ & 6.06 & 13 & -81 & BL \\
\hline Oman et al. & 1977 & $\mathrm{E}$ & MS & 2.78 & 89 & -32 & $\mathrm{BL}$ \\
\hline Lewis et al. & 1977 & $\mathrm{~T}, \mathrm{E}$ & $\mathrm{RB}$ & 2.56 & 148 & -5 & UT \\
\hline Foreman \& Gilbert & 1979 & $\mathrm{E}$ & MS & 2.78 & 130 & -17 & $\mathrm{BL}$ \\
\hline Foreman \& Gilbert & 1979 & $\mathrm{E}$ & $\mathrm{RB}$ & 2.78 & 240 & 22 & $\mathrm{BL}$ \\
\hline Foreman \& Gilbert & 1979 & $\mathrm{E}$ & $\mathrm{RB}$ & 2.78 & 175 & -1 & BL \\
\hline de Vries & 1979 & $\mathrm{~T}$ & $\mathrm{AD}$ & --- & 200 & --- & BL \\
\hline Fletcher & 1981 & $\mathrm{~N}$ & $\mathrm{RB}$ & 4 & 150 & -30 & UT \\
\hline Fletcher & 1981 & $\mathrm{~N}$ & $\mathrm{RB}$ & 6 & 200 & -30 & UT \\
\hline Loeffler & 1981 & $\mathrm{~N}$ & $\mathrm{AD}$ & 4.58 & 760 & 88 & $\mathrm{BL}$ \\
\hline Loeffler & 1981 & $\mathrm{~N}$ & $\mathrm{AD}$ & 3.76 & 280 & 1 & BL \\
\hline
\end{tabular}




\begin{tabular}{|c|c|c|c|c|c|c|c|}
\hline Igra & 1981 & $\mathrm{E}$ & MS & 10.3 & 180 & -73 & $\mathrm{BL}$ \\
\hline Riegler & 1983 & $\mathrm{~T}$ & $\mathrm{AD}$ & 2.78 & 230 & 19 & $\mathrm{BL}$ \\
\hline Foreman \& Gilbert & 1983 & $\mathrm{E}$ & $\mathrm{RB}$ & 2.78 & 175 & 0 & $\mathrm{BL}$ \\
\hline Foreman \& Gilbert* & 1983 & $\mathrm{E}$ & $\mathrm{RB}$ & 2.78 & -27 & -75 & $\mathrm{BL}$ \\
\hline Foreman et al. & 1983 & $\mathrm{E}$ & $\mathrm{RB}$ & 2.62 & 225 & 24 & $\mathrm{BL}$ \\
\hline Foreman et al.* & 1983 & $\mathrm{E}$ & $\mathrm{RB}$ & 2.62 & -36 & -76 & $\mathrm{BL}$ \\
\hline Igra & 1984 & $\mathrm{E}$ & $\mathrm{RB}$ & 4 & 136 & -41 & $\mathrm{BL}$ \\
\hline Dick & 1986 & $\mathrm{~T}$ & $\mathrm{AD}$ & --- & --- & 8 & UT \\
\hline Nagai \& Irabu & 1987 & $\mathrm{~T}$ & $\mathrm{AD}$ & --- & 18 & -35 & $\mathrm{BL}$ \\
\hline Phillips et al. & 1999 & $\mathrm{E}$ & $\mathrm{RB}$ & 3.8 & 300 & 10 & UT \\
\hline Frankovic \& Vrsalovic & 2001 & $\mathrm{E}$ & --- & --- & 228 & --- & UT \\
\hline Bet \& Grassmann & 2003 & $\mathrm{~N}$ & $\mathrm{RB}$ & 2.2 & 60 & -30 & UT \\
\hline Bet \& Grassmann & 2003 & $\mathrm{~N}$ & $\mathrm{RB}$ & 3.4 & 100 & -40 & UT \\
\hline Grassmann et al. & 2003 & $\mathrm{E}$ & $\mathrm{RB}$ & 2.25 & 50 & -30 & UT \\
\hline Grassmann et al. & 2003 & $\mathrm{E}$ & $\mathrm{RB}$ & 2.25 & 100 & -10 & UT \\
\hline Grassmann et al. & 2003 & $\mathrm{~N}$ & $\mathrm{RB}$ & 5.4 & 420 & -4 & UT \\
\hline Phillips & 2003 & $\mathrm{E}$ & MS & 3.41 & 8 & -68 & $\mathrm{BL}$ \\
\hline Phillips & 2003 & $\mathrm{E}$ & MS & 3 & 73 & -42 & $\mathrm{BL}$ \\
\hline Phillips & 2003 & $\mathrm{E}$ & $\mathrm{RB}$ & 3 & 143 & -19 & UT \\
\hline Phillips & 2003 & $\mathrm{E}$ & $\mathrm{RB}$ & 2.22 & 38 & -38 & $\mathrm{BL}$ \\
\hline Abe et al. & 2005 & $\mathrm{~N}, \mathrm{E}$ & $\mathrm{RB}$ & 6.81 & 300 & -42 & UT \\
\hline
\end{tabular}

E: Experimental, T: Theoretical, N: Numerical; RB: Rotor bla6de, AD: Actuator disk, MS: Mesh screen; UT: Unshrouded turbine, BL: Betz limit; * Interpretation of Phillips (2003). 


\subsection{Shrouded Hydrokinetic Turbines}

In this section the literature on hydrokinetic turbine technology is reviewed starting with unshrouded turbines and then focusing on shrouded turbines. Although wind turbine technology has been studied and employed to generate electricity worldwide, extracting energy using hydrokinetic turbines is relatively new and still in its early stages of development. Small numbers of studies are reported in the open literature on hydrokinetic turbines with few studies reporting the effect of shroud. Khan et al. (2009) reviewed different concepts of harnessing the kinetic energy of water streams including turbine and non-turbine systems. The paper reviews existing and future energy conversion methods and different factors involved in the technology such as design, operation, and control systems. Based on the study they carried out on 76 different turbine systems and rotor configurations, horizontal and vertical axis turbines have the most popularity with respective number of application of $43 \%$ and $33 \%$ among all. A good classification was made on hydrokinetic turbines and the augmentation schemes used to enhance the output power of vertical and horizontal axis turbines. The authors concluded substantial research and development still need to be done before hydrokinetic turbine technology could be commercialized. Khan et al. (2008) concentrated on river current energy conversion systems and provided a literature and technology status review on hydrokinetic turbine technology and the challenges it faces. Some challenges are design of the rotor blade and shroud, water sealing, and cavitation of the blades. Applicability of shroud in hydrokinetic turbine technology was mentioned as an advantage compared to wind turbine technology since there is much less need for a yawing system in water streams than in the wind. A shroud can also be used as a 
structure to float the turbine in the water or to install it on the riverbed or as a casing for components of the generator (Drouen et al., 2007). O Rourke et al. (2010) also conducted a literature review on hydrokinetic turbines with a focus on tidal energy extraction techniques. Tidal energy is explained to have two components of potential and kinetic energies based on which tidal power facilities are divided into two categories. A tidal barrage extracts the potential energy and a tidal turbine extracts the kinetic energy of tidal streams. A number of technologies from vertical axis turbines to different shrouded or open center horizontal axis turbines in design stages or in pilot operations were reviewed. The authors based on then existing operational devices assumed horizontal axis turbines would be the optimum system for tidal energy extraction. Several other papers of this nature review current and upcoming technology status and challenges, some with feasibility studies for a particular location (Bahaj, 2011; Güney and Kaygusuz, 2010; Hassan et al., 2012; Lago et al., 2010; Nicholls-Lee and Turnock, 2008; Vermaak et al., 2014; Yuce and Muratoglu, 2015).

Design of the rotor blades, as well as the effect of stall, cavitation, pitch and yaw angles of blades on the performance of horizontal axis hydrokinetic turbines have been studied. Batten et al. (2006) numerically studied effect of certain design parameters of rotor blades such as the airfoil profile and pitch angle on the performance of hydrokinetic turbines. They studied stall and cavitation characteristics for a few airfoil sections using XFoil. XFoil is a linear vorticity stream function panel method code with viscous boundary layer and wake effects. The blade element momentum theory was also used to develop a numerical method to study and compare the performance of rotor blades having two different airfoil profiles and pitch angles. The authors also investigated the 
effect of yaw angle, immersion depth, and an adjacent rotor blade on the performance of an $80 \mathrm{~cm}$ diameter three bladed horizontal axis turbine in a cavitation tunnel and a towing tank (Bahaj et al., 2007b). Results of the numerical and experimental studies were then compared and it was stated the developed numerical method is a useful design and optimisation tool for the turbines energy output (Bahaj et al., 2007a; Batten et al., 2008, 2007). Myers and Bahaj (2006) performed an experimental study on a $40 \mathrm{~cm}$ diameter three bladed horizontal axis turbine in a circulating water channel. They measured the turbine output power for different blade pitch and rotor yaw angles at different flow velocities. Results of a commercial blade element momentum code were also compared to the experimental results and different levels of discrepancy were observed. They concluded further investigations are required on the effect of yaw angle and stall delay on the performance of a hydrokinetic turbine.

In the contrary to diffuser augmented wind turbines, only a few works have been reported in the open literature on shrouded horizontal axis hydrokinetic turbines. In the absence of a consistent theory for choosing an inlet and outlet for a shrouded hydrokinetic turbine (Lawn, 2003), different designs with different yet inconsistent results on the effectiveness of a shroud are reported. Lawn (2003) investigated shrouded hydrokinetic turbines using a one-dimensional theory that treats a duct as a contraction or an expansion upstream or downstream of a turbine. He showed more than $30 \%$ enhancement over the optimum performance of an unshrouded turbine can be gained with a shroud. He concluded the significant power enhancement reported in the literature is associated with non-optimum conditions for unshrouded turbines. Gaden (2007) studied the power enhancement of shrouded hydrokinetic turbines using three dimensional CFD simulations. He simulated 
the rotor blade using the rotating reference frame model and tested different configurations for the shroud model. A shroud with a cylindrical inlet and a $20^{\circ}$ half angle diffuser having an exit area ratio of 1.56 was found to enhance the turbine output power by a factor of 1.5. Comparing a few shroud models he later stated a cylindrical inlet degrades the turbine performance. Gaden and Bibeau (2010) using the commercial code ANSYS CFX simulated the turbine rotor blade as a momentum source region and found an enhancement factor of 3.1 for the same shroud used in the previous study. The inconsistency of the enhancement factors obtained in the two studies is stated to be due to the different methods used to simulate the rotor blade. Scherillo et al. (2011) performed experimental and numerical studies on shrouded hydrokinetic turbines. The geometry of the shroud which was a diffuser having an airfoil cross section with a $26^{\circ}$ pitch angle and an exit to throat area ratio of 1.7 was obtained in a numerical optimization process. The wind tunnel and towing tank experiments showed the shroud enhances the maximum power coefficient of the turbine by a factor of 2 which is a $7.5 \%$ increase when the power coefficient is calculated using the diffuser exit diameter. Effect of yaw angle on the performance of the shrouded turbine was also examined and it was observed that the turbine power does not change with yaw angles less than $10^{\circ}$. Coiro et al. (2012) conducted field experiments on a full scale Ocean's kite system made to harness the kinetic energy of tidal currents. The system consisted of two counter rotating rotor blades installed on a torpedo shape body which is tethered to the seabed. They used the shroud model of the previous study to increase the output power of the system. The authors concluded the system is feasible and reliable for generating electricity from water streams particularly with shrouded turbines. Shives and Crawford (2011) performed axi- 
symmetric CFD simulations on a series of shrouds with the turbine rotor modeled as an actuator disk. They studied the effect of viscous loss, flow separation, and base pressure on the performance of the shrouds. They found negligible viscous loss in the inlet and concluded the flow separation in the diffuser section leads to a significant performance loss. They proposed a well-designed duct as a flanged diffuser that creates a large base pressure at the exit. Lokocz (2012) conducted experimental studies on a shrouded horizontal axis hydrokinetic turbine in a towing tank. He used two different rotor blade designs for shrouded and unshrouded turbines and a cylindrical shroud with a NACA 4412 aerofoil cross section. The experimental results showed a slight reduction in the performance of the shrouded turbine. The reason is stated to be the low thrust coefficient of the shroud due to its non-optimum design. Also, no significant change was seen in the performance of the rotor blade used in the shrouded turbine with and without the shroud.

In the area of commercial application diverse designs of shrouded turbines are observed. A number of companies have shrouded hydrokinetic turbines in field tests for tidal stream and river applications. Clean Current Power System Inc. develops river and tidal current turbines for different water velocities and depths. The largest river turbine with $3.5 \mathrm{~m}$ diameter three bladed rotor has a rated power of $65 \mathrm{~kW}$ at a $3 \mathrm{~m} / \mathrm{s}$ water velocity. The turbine utilizes a $3.1 \mathrm{~m}$ long shroud that has a $3.5 \mathrm{~m}$ diameter cylindrical inlet and a $3.5 \mathrm{~m}$ high by $4.6 \mathrm{~m}$ wide oval outlet. The tidal turbines are equipped with a central yaw bearing to follow the flow direction in the flow and ebb tides. Clean Current has currently deployed and is testing a turbine at the Canadian Hydrokinetic Turbine Test Center on the Winnipeg River. OpenHydro manufactures open centre tidal turbines with 
an integrated generator placed in the shroud. The low speed rotating rotor along with the lubricant-free operation of the turbine minimise the risks to marine life. A $6 \mathrm{~m}$ diameter unit is capable of providing enough energy for 150 average homes and prevent emission of 450 tonnes of $\mathrm{CO}_{2}$ per year. They deployed a $1 \mathrm{MW}$ unit in the Bay of Fundy in 2009. Renewable Energy Research fabricates shrouded turbines under the name of TREK. TREK-I turbine has a $340 \mathrm{~kW}$ maximum power output with a $3.6 \mathrm{~m}$ diameter and $7.5 \mathrm{~m}$ long shroud weighing $36 \mathrm{t}$. The turbine needs a minimum $5.5 \mathrm{~m}$ water depth for installation and has a cut-in speed of $1.5 \mathrm{~m} / \mathrm{s}$. RER deployed a TREK-I unit in the Saint Laurence River in 2010. Lunar Energy proposed a bi-directional tidal turbine with a symmetrical Venturi duct known as Rotech Tidal Turbine. A $1 \mathrm{MW}$ unit has an $11.5 \mathrm{~m}$ diameter rotor blade with a $19.2 \mathrm{~m}$ long shroud and weighs $2500 \mathrm{t}$. It is designed to sit on the seabed at a depth of more than $40 \mathrm{~m}$. They designed and patented a $2.4 \mathrm{MW}$ unit in 2012.

It is worth mentioning the idea of enhancing the turbine performance by means of a shroud has not been restricted to horizontal axis turbines. Ponta and Jacovkis (2008) proposed a channelling device consisted of two floating pontoons embedding a vertical axis Darrieus turbine. They tested the device in a towing tank and observed a flow speed increase in the channel based on which they concluded the device would increase the turbine output power. Kirke (2011) reported on field experiments performed on a number of helical and straight Darrieus turbines with fixed and variable pitch blades. Tests were done on shrouded and unshrouded turbines mounted in front of a barge moving in the river. The diffuser was observed to triple the output power in one turbine configuration among all. The present author's point of view is that although a shroud improves the 
performance of a vertical turbine as a result of elevated flow velocity, the turbine is no longer insensitive to changes in the flow direction. The insensitivity of vertical axis turbines to flow direction is an advantage over horizontal axis turbines.

\subsection{Yaw Operation}

Evaluating the performance in yaw operations has been part of research on wind turbines. Smulders et al. (1981) measured the power and thrust force of a $20 \mathrm{~cm}$ diameter two bladed horizontal axis wind turbine in off-axis operations in an open $50 \mathrm{~cm}$ by $50 \mathrm{~cm}$ wind tunnel. They found for yaw angles as high as $45^{\circ}$, the optimum tip speed ratio does not change while the power coefficient decreases significantly. They observed the performance coefficient shows cosine cubed dependence to yaw angle as predicted by the linear momentum theory. The cosine cubed dependence of the performance to yaw angle was also observed by Schumack (1979) in the experiments on a different turbine. Anderson et al. (1982) performed experiments on a $3 \mathrm{~m}$ diameter two bladed horizontal axis wind turbine. They did wind tunnel and field experiments with blades having a NACA 4412 airfoil profile. The turbine made $12 \%$ blockage in the $9.2 \mathrm{~m}$ by $6.5 \mathrm{~m}$ wind tunnel. This was considered a reasonably low blockage ratio and the Glauert (1947) relation was used to correct for blockage effects. The Glauert relation is shown to be adequately accurate for small blockage ratios (Willmer, 1979). They examined the Reynolds number effect on the turbine performance and found the power coefficient to increase with increasing Reynolds number. They also observed in agreement with the linear momentum theory the power coefficients in yaw operations decrease with cosine cubed of yaw angle. 
Grumman Corp. in an experiment used the baseline diffuser and the two bladed $457 \mathrm{~mm}$ diameter turbine to assess the performance of diffuser augmented wind turbines in yawed flow conditions (Foreman and Gilbert, 1983; Foreman et al., 1983). They found the unshrouded turbine insensitive to yaw angles less than $30^{\circ}$ but observed a sudden $80 \%$ increase in the turbine power at $30^{\circ}$ yaw angle. They also observed negligible changes in the power of the diffuser augmented wind turbine up to $30^{\circ}$ yaw angle followed by an abrupt reduction in the power at $40^{\circ}$ yaw angle. They speculated a sufficiently large inlet contraction reduces the power sensitivity to yawed flows. However, they believed their yaw tests to be inconclusive. The reason is that it was not possible to determine whether the yaw insensitivity is attributed to the effect of the long upstream nacelle channelling the flow into the rotor blade or it is the effect of the diffuser itself.

Grant et al. (1997) conducted experiments on a $90 \mathrm{~cm}$ diameter horizontal axis wind turbine in an open jet wind tunnel. Two and three bladed configurations of the turbine were tested. Power coefficients and wake of the turbine were examined in inline and offaxis flows up to $30^{\circ}$ yaw angles. The ratio of the maximum power coefficient in off-axis flows to the maximum power coefficient in zero yaw were compared to the experimental data of Clayton and Filby (1982). The power coefficients in yaw operations from the two datasets closely follow the pattern of cosine cubed of yaw angle. The slightly higher power coefficients Grant el al. obtained is explained to be due to the electrical losses in the wind turbine circuit.

Coton and Wang (1999) studied the performance of a horizontal axis wind turbine in yawed flows. They used a numerical method that couples a prescribed wake vortex 
model and the Leishman-Beddoes semi-empirical dynamic stall model (Leishman and Beddoes, 1989). The calculated results showed a general agreement with experimental data. Considering the shortcomings of the model, the authors noted the need for extensive experimental data on yawed turbines that can be employed in the empirical models, integrated into the numerical models, and used to validate the numerical simulations.

National Renewable Energy Laboratory, NREL, has conducted field measurements and wind tunnel experiments on a $10 \mathrm{~m}$ diameter two bladed horizontal axis wind turbine using S809 airfoil profile since 1987. A comprehensive set of wind tunnel experiments were performed on a heavily instrumented wind turbine in $24.4 \mathrm{~m}$ by $36.6 \mathrm{~m}$ NASA wind tunnel (Hand et al., 2001). The experiments provided accurate quantitative aerodynamic and structural measurements on a wind turbine which geometrically and dynamically represents a full-scale wind turbine. The power and thrust force as well as pressure, structural moments and loads on the blades were measured and reported for the turbine operating in yawed conditions. The maximum power and the thrust at the maximum power show the decreasing trend predicted by the linear momentum theory. It is observed that the maximum power coefficient decreases with cosine cubed of yaw angle and the thrust coefficient at the maximum power decreases with cosine squared of yaw angle.

Maeda et al. (2008) performed wind tunnel experiments and measured the power and pressure distribution of a 2.4 m diameter horizontal axis wind turbine in yaw operations. Power and torque curves were obtained for a range of tip speed ratio and yaw angle in two directions. Identical performance coefficients were obtained for the two directions of yawing in the horizontal plane. It is observed that the ratio of power coefficients in yaw 
operations to those in zero yaw angle decreases with cosine cubed of yaw angle. A small difference between the experimental data and the theory prediction occurs at larger yaw angles.

Adaramola and Krogstad (2011) experimentally investigated the effect of a wind turbine wake on the performance of a downstream turbine in a wind turbine farm. Two $90 \mathrm{~cm}$ diameter three bladed horizontal axis wind turbines were used in wind tunnel experiments. Power and thrust curves of a single turbine were obtained for a range of yaw angle later published in (Krogstad and Adaramola, 2012). In agreement with the linear momentum theory, the measured power is observed to decrease with cosine cubed of yaw angle. They also measured the performance of the downstream turbine when placed at different distances from the upstream turbine operating off-axis. They observed the performance of the downstream turbine improves when the upstream turbine is yawed and away from the optimum performance. The authors concluded operating an upstream turbine at an appropriate yaw angle and losing a controlled certain amount of performance results in an increase in the overall efficiency of a wind farm. This consequently reduces the space requirement for a wind farm.

Noura et al. (2012) examined a $50 \mathrm{~cm}$ diameter three bladed horizontal axis wind turbine in a wind tunnel. The performance curve of the turbine and the downstream velocity field using PIV measurement were obtained at different yaw angles. In agreement with the previous studies and the linear momentum theory they observed the reduction of the power in yawed flows is proportional to cosine cubed of yaw angle. Tsalicoglou et al. (2012) studied the effect of flow inclination on the performance of a wind turbine. 
Performance and flow field measurements were conducted on a $30 \mathrm{~cm}$ diameter three bladed turbine in a towing tank. Performance coefficients of the turbine in yaw angles up to $40^{\circ}$ were measured and observed to decrease with cosine cubed of yaw angle. Smaller performance reduction was found in yawed flows with higher turbulence intensities. They considered the surface and blockage effects negligible based on a previous study done in the same facility (Barber et al., 2011). Barber et al. (2011) stated the 7.1\% blockage ratio in the experiments is less than the $7.5 \%$ limit introduced by Barlow et al. (1999) below which blockage correction is not required. 


\section{Chapter 3 Theoretical Analysis}

\subsection{Foreword}

Characteristics of a wind turbine have been traditionally studied using the linear momentum theory. Despite the ideal assumptions it makes in analyzing the power and thrust of a turbine, it provides a good understanding of the behaviour of kinetic turbines. In the next three sections of this chapter the application of the linear momentum theory to a hydrokinetic turbine in aligned and off-axis flows, as well as to the inline operations of a shrouded turbine is reviewed.

\subsection{The Linear Momentum Theory}

In analysing wind turbines a simple model based on the linear momentum theory is used to determine the power output and thrust force of an ideal wind turbine. This model was first used over 100 years ago to predict the performance of ship propellers. Later, a German aerodynamicist employed the model to analyze the performance characteristics of a wind turbine (Betz, 1926). The turbine rotor is modeled by a circular disk or an "actuator disk" across which the flow experiences a sudden pressure drop and a continuous velocity reduction. The model is known as the actuator disk model and is 
applicable to any kind of wind turbine. A streamtube enclosing the disk along with the two cross sections far upstream and far downstream of the disk, as shown in Figure 3.1, forms the control volume of the analysis. Flow enters the control volume from the upstream boundary and exits from the downstream boundary and there is no flow across the streamtube surface. A few assumptions are made in the model to simplify the analysis:

- flow is steady, homogeneous, and incompressible

- $\quad$ fluid is inviscid

- turbine rotor has infinite number of blades

- pressure and velocity distributions are uniform over the actuator disk

- wake behind the turbine rotor does not rotate

- far upstream and far downstream of the rotor have ambient static pressure

The flow velocity gradually decreases as the fluid moves toward the actuator disk and becomes less than the ambient velocity at the disk. Reduction in the flow velocity results in the reduction of the flow kinetic energy which in return, gives rise to its static pressure. Static pressure of the fluid suddenly drops below the ambient pressure while the flow passes the actuator disk. Thrust force on the disk is uniform as a result of the assumed uniform pressure distribution. The fluid behind the actuator disk with a reduced velocity and static pressure slowly moves downstream. This is known as the turbine wake. Far downstream of the disk, the flow needs to reach the ambient pressure. This happens by further decrease in the kinetic energy and velocity of the flow. Far upstream and far downstream of the disk have the same ambient pressure at the expense of the reduction in the kinetic energy of the flow. 


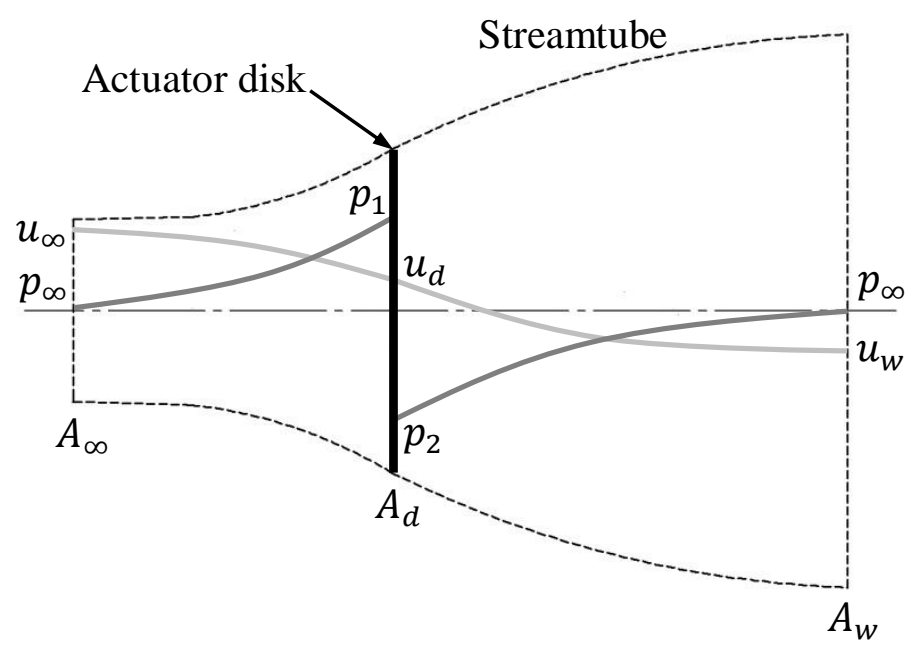

Figure 3.1: Control volume over the actuator disk in analysing a wind turbine. Flow velocity undergoes a continuous decrease while pressure drops suddenly across the disc.

Taking the assumptions of the actuator disk model into account, conservation of mass and linear momentum can be written as follows:

$$
\begin{gathered}
\int_{C S} \rho \vec{V} \cdot d \vec{A}=0, \\
\int_{C S} \vec{V} \rho \vec{V} \cdot d \vec{A}=\vec{F} .
\end{gathered}
$$

The thrust force exerted on the disk is found by applying the linear momentum equation, Equation (3.2), to the control volume over the actuator disk, Figure 3.1. Thrust force is the rate of change of the flow momentum in the control volume:

$$
T=\rho u_{\infty}^{2} A_{\infty}-\rho u_{w}^{2} A_{w} .
$$

From the principle of conservation of mass, Equation (3.1) shows for an incompressible flow the mass flow rate and the volume flow rate are constant throughout the control volume: 


$$
u_{\infty} A_{\infty}=u_{w} A_{w}=u_{d} A_{d} .
$$

Using Equation (3.4) into Equation (3.3), the thrust force can be written as:

$$
T=\rho u_{d} A_{d}\left(u_{\infty}-u_{w}\right)
$$

in which the fluid density is denoted by $\rho$, the flow velocity by $\mathrm{u}$, and the disk area by A. The subscripts denote the locations indicated in Figure 3.1. The thrust force can also be calculated from the net pressure force acting on the actuator disk:

$$
T=\left(p_{1}-p_{2}\right) A_{d}
$$

Pressure on the actuator disk can be found by applying the Bernoulli's equation to the separate control volumes on either side of the disk. The Bernoulli's equation cannot be applied to a control volume containing the actuator disk as work is done on the disk. Applying the Bernoulli's equation to the control volumes upstream and downstream of the actuator disc gives:

$$
\begin{aligned}
& p_{\infty}+\frac{1}{2} \rho u_{\infty}^{2}=p_{1}+\frac{1}{2} \rho u_{d}^{2}, \\
& p_{2}+\frac{1}{2} \rho u_{d}^{2}=p_{\infty}+\frac{1}{2} \rho u_{w}^{2} .
\end{aligned}
$$

Subtracting Equation (3.8) from (3.7) and inserting the result in Equation (3.6) along with the assumption that the flow velocity across the disk remains constant gives:

$$
T=\frac{1}{2} \rho A_{d}\left(u_{\infty}^{2}-u_{w}^{2}\right) .
$$


Comparing Equations (3.5) and (3.9), the flow velocity at the disk is found to be the average of the upstream and downstream velocities:

$$
u_{d}=\frac{1}{2}\left(u_{\infty}+u_{w}\right)
$$

The relative decrease between the free stream velocity and the flow velocity at the disk is the axial flow induction factor or the inflow factor:

$$
a=\frac{u_{\infty}-u_{d}}{u_{\infty}}
$$

The difference between the free stream velocity and the flow velocity at the disk, $a u_{\infty}$, is referred to as the induced velocity at the disk. The flow velocity at the disk and at the wake can be written in terms of the axial induction factor:

$$
\begin{gathered}
u_{d}=u_{\infty}(1-a), \\
u_{w}=u_{\infty}(1-2 a) .
\end{gathered}
$$

The flow velocity at the wake decreases as the induction factor increases where at $a=1 / 2$ the velocity at the wake becomes zero, Equation (3.13), and the momentum theory is no longer applicable. Further increase in the induction factor results in the negative velocity at the far wake. A horizontal axis wind turbine in this situation operates in the "turbulent wake state" and more advanced models with empirical modifications need to be used (Eggleston and Stoddard, 1987).

The rate at which work is done on the disk, i.e. the power, is then found by multiplying the thrust force by the flow velocity at the disk: 


$$
P=T u_{d}=2 \rho u_{\infty}^{3} A_{d} a(1-a)^{2} .
$$

Performance of a wind turbine is usually characterized by a non-dimensional coefficient known as the power coefficient or the performance coefficient. Power coefficient is the ratio of the rotor output power to the power available in the flow for the rotor area:

$$
C_{P}=\frac{P}{\frac{1}{2} \rho u_{\infty}^{3} A_{d}}=4 a(1-a)^{2} .
$$

The thrust force on the rotor can also be represented by a non-dimensional thrust coefficient as follows:

$$
C_{T}=\frac{T}{\frac{1}{2} \rho u_{\infty}^{2} A_{d}}=4 a(1-a) .
$$

The maximum power coefficient $C_{P_{\max }}=16 / 27=0.59$ occurs at an axial induction factor of $a=1 / 3$ and is known as the Betz limit (Betz, 1920). The thrust coefficient at the maximum power is $C_{T}=8 / 9$ obtained from Equation (3.16). Complicated flow patterns occur at the higher induction factors exceeding 0.5 and the thrust coefficient goes as high as 2.0. In this situation, the linear momentum theory is no longer valid and empirical relations should be used to find the thrust coefficient (Wilson et al., 1976).

The Betz limit is the maximum theoretical value for the performance of a kinetic turbine and shows the upper limit for the power extraction from a fluid flow. No kinetic turbine is able to reach that limit regardless of the turbine design. Some of the assumptions made in the model to obtain this limit are close to the practical conditions for a kinetic turbine while some are ideal assumptions and cannot be reached in the reality. Inviscid fluid, 
infinite number of blades, uniform thrust over the actuator disk, and non-rotating wake are the ideal assumptions. These ideal assumptions make the Betz limit an ideal theoretical value that gives the upper limit for the kinetic power extraction. Aerodynamic and mechanical losses in the real facilities added to this reason prevent a turbine performance to approach the Betz limit (Wilson, 1980).

\subsection{The Momentum Theory in Yaw Operations}

The linear momentum theory is unable to account for variations of the induced velocity on the rotor disk and only provides an average value for the induced velocity on the entire disk. Unlike the inline flow situation where a uniform induced velocity can be assumed over the actuator disc, the induced velocity on a rotor disc at yaw angle varies both radially and azimuthally. Therefore, application of the linear momentum theory to a rotor disc in yaw operations is controversial (Burton et al., 2001). There is more uncertainty in the basic calculations, like determining the average rotor power, of yawed flows than aligned flows (Jamieson, 2011). In the linear momentum theory, the thrust force on the actuator disc is assumed responsible for the rate of change of momentum of the flow. This force results from the pressure difference on the disk and is normal to the disk plane and hence the induced velocity is also in the same axial direction. The component of the induced velocity normal to the incoming off-axis flow deflects the wake to one side and the wake is no longer symmetric about the rotor axis as shown in Figure 3.2. The effect of yaw angle, as a simplifying assumption, is modeled by taking the angle of the inflow velocity vector into account (Manwell et al., 2009). Applying the 
principles of conservation of mass and momentum, Equations (3.1) and (3.2), to a control volume enclosing the whole system for a steady flow results in:

$$
\begin{aligned}
& u_{\infty} \cos \gamma A_{\infty}=u_{w} A_{w}=u_{d} A_{d}, \\
& T=\rho u_{\infty}^{2} \cos ^{2} \gamma A_{\infty}-\rho u_{w}^{2} A_{w} .
\end{aligned}
$$

Equation (3.18) can be written as follows using Equation (3.17):

$$
T=\rho u_{d} A_{d}\left(u_{\infty} \cos \gamma-u_{w}\right)
$$

Applying the Bernoulli's equation to either side of the actuator disk gives:

$$
\begin{gathered}
p_{\infty}+\frac{1}{2} \rho u_{\infty}^{2} \cos ^{2} \gamma=p_{1}+\frac{1}{2} \rho u_{1}^{2}, \\
p_{2}+\frac{1}{2} \rho u_{2}^{2}=p_{\infty}+\frac{1}{2} \rho u_{w}^{2} .
\end{gathered}
$$

Subtracting Equation (3.21) from (3.20) along with the assumption that the velocity across the disk remains constant we have:

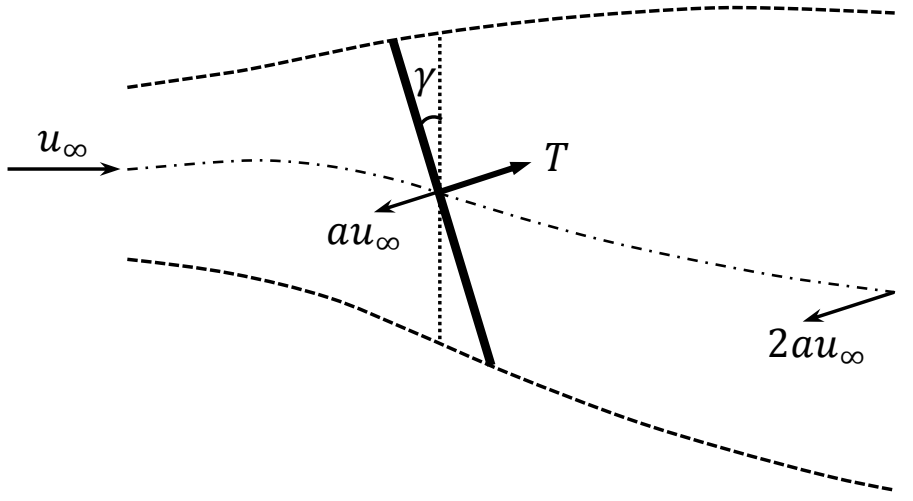

Figure 3.2: Deflected wake of a yawed turbine and the induced velocities. The induced velocity at the wake is twice the induced velocity at the disk. 


$$
T=\left(p_{1}-p_{2}\right) A_{d}=\frac{1}{2} \rho A_{d}\left(u_{\infty}^{2} \cos ^{2} \gamma-u_{w}^{2}\right) .
$$

Comparing Equations (3.19) and (3.22) we find, as in the inline flow, that the flow velocity at the disk is the average of the upstream and downstream flow velocities:

$$
u_{d}=\frac{1}{2}\left(u_{\infty} \cos \gamma+u_{w}\right)
$$

Defining the axial induction factor as:

$$
a=\frac{u_{\infty} \cos \gamma-u_{d}}{u_{\infty}},
$$

the flow velocity at the disk and at the wake are written as:

$$
\begin{gathered}
u_{d}=u_{\infty}(\cos \gamma-a), \\
u_{w}=u_{\infty}(\cos \gamma-2 a),
\end{gathered}
$$

where the average induced velocity at the wake, as in the inline flow, is twice the induced velocity at the disk. Substituting Equation (3.26) into (3.22), the thrust force can be written as:

$$
T=2 \rho u_{\infty}^{2} A_{d} a(\cos \gamma-a) .
$$

Using Equation (3.25), the output power becomes:

$$
P=T u_{d}=2 \rho u_{\infty}^{3} A_{d} a(\cos \gamma-a)^{2} .
$$

The thrust and power coefficients for a yawed turbine are then calculated as: 


$$
\begin{gathered}
C_{T}=\frac{T}{\frac{1}{2} \rho u_{\infty}^{2} A_{d}}=4 a(\cos \gamma-a), \\
C_{P}=\frac{P}{\frac{1}{2} \rho u_{\infty}^{3} A_{d}}=4 a(\cos \gamma-a)^{2} .
\end{gathered}
$$

Solving for the maximum power coefficient we get:

$$
C_{P \max }=\frac{16}{27} \cos ^{3} \gamma
$$

which occurs at an induction factor of $a=\cos \gamma / 3$. The thrust coefficient at the maximum power is then $C_{T}=8 / 9 \cos ^{2} \gamma$.

Application of the momentum theory to off-axis flows using the axial component of the flow velocity is questionable since the lateral pressure gradients deflect the wake and impact the axial induced velocity. Where an accurate detail calculation of the blade forces is necessary, advanced aerodynamic modelling using vortex wake models or CFD analysis are used (Leishman, 2002). It nevertheless, gives satisfactory results for the average axial induction factor and rotor power. $\cos ^{3} \gamma$ appearing in Equation (3.31) has been accepted as the rule of thumb in assessing the performance reduction of unshrouded wind turbines in yaw operations (Burton et al., 2001).

\subsection{The van Bussel Momentum Theory for Shrouded Turbines}

A similar approach has been used by van Bussel $(2007,1999)$ in studying diffuser augmented wind turbines. He uses the assumptions of the actuator disk model and writes 
the momentum equation to analyze the performance of a shrouded wind turbine. The van Bussel momentum theory for shrouded turbines is explained in this section.

Velocity and pressure in an empty diffuser are directly related to its geometry. Pressure relations in an empty diffuser assuming an inviscid flow can be written as follows:

$$
\begin{aligned}
& p_{\infty}+\frac{1}{2} \rho u_{\infty}^{2}=p_{e}+\frac{1}{2} \rho u_{e}^{2}, \\
& p_{\infty}+\frac{1}{2} \rho u_{\infty}^{2}=p_{1}+\frac{1}{2} \rho u_{1}^{2}
\end{aligned}
$$

where $p_{\infty}$ is pressure at the far upstream and at the far wake of the diffuser. The diffuser exit area ratio, EAR, can be written in terms of the velocity ratio using the continuity equation:

$$
E A R=\frac{A_{e}}{A_{d}}=\frac{u_{d}}{u_{e}} .
$$

Assuming no back pressure at the diffuser exit and thus assuming $p_{e}=p_{\infty}$ and $u_{e}=u_{\infty}$, pressure at the diffuser throat can be written as:

$$
p_{1}=p_{\infty}+\left(1-E A R^{2}\right) \frac{1}{2} \rho u_{\infty}^{2}
$$

When the diffuser exit area ratio is larger than unity there exists an under pressure at the throat. When the flow is forced to deflect in the radial direction, like in the case of a flanged diffuser, a back pressure may exist at the diffuser exit and the exit velocity is no longer equal to the uniform upstream velocity, $u_{\infty}$. Introducing back pressure by the velocity ratio, $\eta=u_{e} / u_{\infty}$, we write pressure at the throat as follows: 


$$
p_{1}=p_{\infty}+\left(1-E A R^{2} \eta^{2}\right) \frac{1}{2} \rho u_{\infty}^{2}
$$

Therefore, for an exit area ratio larger than unity and for a zero or negative back pressure there exists an under pressure at the throat. Back pressure is the pressure of the surroundings downstream of the diffuser exit and a zero or negative back pressure occurs when $p_{e} \leq p_{\infty}$ and hence $\eta \geq 1$.

In many cases the reason for over-predicting the achievable power of diffuser augmented wind turbines is the high velocity ratio of the flow at the throat and at the far upstream for an empty diffuser. For a diffuser augmented wind turbine the velocity at the inlet depends on the actual geometry of the diffuser. However, with the assumption of a smooth inflow and no separation, the Bernoulli's equation helps understanding the energy conversion process. The velocity at the diffuser exit $u_{e}$ is lower than the ambient velocity $u_{\infty}$. Downstream of the diffuser exit, the flow velocity reduces to the wake velocity $u_{w}$ at the ambient pressure $p_{\infty}$. The velocity at the diffuser exit and at the far wake behind a diffuser augmented wind turbine is written as follows:

$$
\begin{gathered}
u_{e}=u_{\infty}(1-\bar{a}), \\
u_{w}=u_{\infty}(1-2 \bar{a}),
\end{gathered}
$$

where $\bar{a}$ is the axial induction factor of a diffuser augmented wind turbine defined as:

$$
\bar{a}=\frac{u_{\infty}-u_{e}}{u_{\infty}}
$$

The flow velocity at the rotor disk at the diffuser throat can be calculated by the continuity equation as: 


$$
u_{d}=u_{\infty}(1-\bar{a}) E A R .
$$

An extra back pressure could happen when the local flow conditions do not match the flow expansion at the diffuser exit through the Kutta condition. The flow velocity in this situation becomes:

$$
u_{e}=u_{\infty}(1-\bar{a}) \eta
$$

Employing the continuity equation along with Equation (3.41) yields the flow velocity at the actuator disk, i.e. the turbine rotor:

$$
u_{d}=u_{\infty}(1-\bar{a}) \eta E A R .
$$

Writing the Bernoulli's equation at the diffuser exit and using Equation (3.37) we have:

$$
p_{e}+\frac{1}{2} \rho u_{e}^{2}=p_{e}+(1-\bar{a})^{2} \frac{1}{2} \rho u_{\infty}^{2} .
$$

Applying the Bernoulli's equation between the diffuser exit and the wake with the assumption that the flow behind the diffuser develops the same as the flow behind an unshrouded turbine and using Equation (3.38) we get:

$$
p_{e}+\frac{1}{2} \rho u_{e}^{2}=p_{\infty}+(1-2 \bar{a})^{2} \frac{1}{2} \rho u_{\infty}^{2} .
$$

From Equations (3.43) and (3.44), pressure at the diffuser exit becomes:

$$
p_{e}=p_{\infty}+\left(3 \bar{a}^{2}-2 \bar{a}\right) \frac{1}{2} \rho u_{\infty}^{2}
$$

which for the optimum performance at $\bar{a}=1 / 3$ yields: 


$$
p_{e}=p_{\infty}-\frac{1}{3} \frac{1}{2} \rho u_{\infty}^{2}
$$

This shows placing a rotor blade inside a diffuser creates a back pressure at the diffuser exit with an amount equal to the under pressure found at the wake of an unshrouded wind turbine. When an extra back pressure exists at the diffuser exit, the Bernoulli's equation and Equation (3.41) give:

$$
p_{e}+\frac{1}{2} \rho u_{e}^{2}=p_{e}+(1-\bar{a})^{2} \eta^{2} \frac{1}{2} \rho u_{\infty}^{2}
$$

The Bernoulli's equation can be applied inside the diffuser at either side of the actuator disk. Applying the Bernoulli's equation upstream of the disk and using Equation (3.42) yields:

$$
p_{\infty}+\frac{1}{2} \rho u_{\infty}^{2}=p_{1}+(1-\bar{a})^{2} \eta^{2} E A R^{2} \frac{1}{2} \rho u_{\infty}^{2}
$$

which gives the pressure at the immediate upstream of the disk:

$$
p_{1}=p_{\infty}+\left[1-(1-\bar{a})^{2} \eta^{2} E A R^{2}\right] \frac{1}{2} \rho u_{\infty}^{2}
$$

Similarly, writing the Bernoulli's equation for the downstream of the disk along with using Equations (3.38) and (3.42) gives:

$$
p_{\infty}+(1-2 \bar{a})^{2} \frac{1}{2} \rho u_{\infty}^{2}=p_{2}+(1-\bar{a})^{2} \eta^{2} E A R^{2} \frac{1}{2} \rho u_{\infty}^{2}
$$

which gives the pressure at the immediate downstream of the actuator disk: 


$$
p_{2}=p_{\infty}+\left[(1-2 \bar{a})^{2}-(1-\bar{a})^{2} \eta^{2} E A R^{2}\right] \frac{1}{2} \rho u_{\infty}^{2}
$$

For the optimal condition at $\bar{a}=1 / 3$, this yields:

$$
p_{2}=p_{\infty}+\left[\frac{1}{9}-\frac{4}{9} \eta^{2} E A R^{2}\right] \frac{1}{2} \rho u_{\infty}^{2}
$$

Subtracting Equation (3.51) from Equation (3.49) gives the pressure drop over the actuator disk as:

$$
p_{1}-p_{2}=4 \bar{a}(1-\bar{a}) \frac{1}{2} \rho u_{\infty}^{2}
$$

which is independent of the diffuser area ratio and back pressure ratio. This indicates the amount of energy extracted per unit volume is the same as that extracted by an unshrouded turbine. However, the amount of air passing through the turbine rotor, because of the diffuser, is increased by the factor $\eta \cdot E A R$ compared to an unshrouded turbine of the same rotor size. In other words, a diffuser provides a larger fluid volume per unit time which results in a larger amount of power available. From Equations (3.53) and (3.16) at $\bar{a}=1 / 3$ we get a thrust coefficient of $8 / 9$ at the maximum power for a shrouded turbine (Jamieson, 2011).

Power coefficient of a shrouded turbine based on the rotor area can be calculated from Equations (3.53) and (3.42):

$$
C_{P}=\frac{\left(p_{1}-p_{2}\right) A_{d} u_{d}}{\frac{1}{2} \rho u_{\infty}^{3} A_{d}}=4 \bar{a}(1-\bar{a})^{2} \eta E A R
$$

Calculating the power coefficient based on the diffuser exit area gives: 


$$
C_{P_{\text {Dif }}}=4 \bar{a}(1-\bar{a})^{2} \eta .
$$

The maximum power, as in the case of an unshrouded turbine, occurs at $\bar{a}=1 / 3$.

Clearly, the van Bussel momentum theory does not predict the maximum achievable back pressure ratio that can be obtained in experiments (van Bussel, 1999). van Bussel (2007) reviewed several experimental studies available in the literature and concluded large values of back pressure have been achieved with no reasonable diffuser performance. The maximum power coefficient he reported is 0.47 based on the diffuser exit area which is way lower than what is expected from a diffuser augmented wind turbine. The van Bussel momentum theory shows the amount of energy extracted per unit volume with a shrouded and an unshrouded turbine is the same. This means the Betz limit is applicable also to shrouded turbines provided that the performance coefficient is calculated based on the diffuser exit area. 


\section{Chapter 4 Methodology of the Study}

\subsection{Foreword}

Wind and water tunnel experiments using scaled prototypes provide detailed data in studying wind and hydrokinetic turbines. Two problems associated with wind and water tunnel experiments on model turbines are scaling and blockage effects. Wind and water tunnel tests, in spite of these limitations, are still preferred to field measurements (Adaramola and Krogstad, 2011). The incoming flow in a field test is much more complicated and difficult to describe in sufficient detail. Calculations are then very timeconsuming as a result of the unsteady characteristics of water streams and specially the wind (de Vries, 1983). Full scale tests are also more expensive to perform and rarely provide all required information for a well-defined CFD simulation. Wave induced motion in field tests of a tidal turbine or a floating offshore wind turbine adds even more complexity to the problem. Wind and water tunnel tests on model turbines are therefore essential due to the controlled test conditions that can be generated in the in-laboratory experiments (Vermeer et al., 2003). Change in the variables that influence the performance of a turbine and its wake can be adjusted and controlled in the tunnel tests.

This study aims to experimentally quantify the effect of a shroud on the performance characteristics of a hydrokinetic turbine in inline and off-axis operations. This chapter 
describes the model horizontal axis hydrokinetic turbine as well as two shrouds designed and fabricated for this study. The measurement procedure employed to perform water tunnel experiments is also explained. The tests are done at three water velocities and six flow angles for shrouded and unshrouded turbine configurations. The output power of the model turbine and its thrust force are measured experimentally. Scaling and blockage effects associated with water tunnel experiments are addressed. The results are corrected for free surface proximity and blockage effects. Validity of the results for large scale turbines at higher Reynolds numbers is also discussed.

\subsection{Experimental Setup}

Components of the experimental setup designed and fabricated by the present author to fulfil the objectives of the study as well as the details of the facilities and equipment used in the experiments are explained in this section. A more robust design of the turbine and repeatable results required after obtaining experimental results from the first turbine model (Shahsavarifard and Bibeau, 2013) led the author to design and fabricate the second model turbine details of which are described in this section.

\subsubsection{Water Tunnel}

The experiments are performed in a recirculating water tunnel facility at the University of Manitoba. The tunnel has a vertical flow loop configuration with a $61 \mathrm{~cm}$ wide by $183 \mathrm{~cm}$ long test section which allows a maximum $60 \mathrm{~cm}$ of water height in the test section. A single stage, axial flow, propeller pump circulates water in the water tunnel at 
a maximum flow rate of $362 \mathrm{~L} / \mathrm{s}$. This provides a maximum water velocity of $1.1 \mathrm{~m} / \mathrm{s}$ at the test section for the full water height. A $30 \mathrm{HP} 3 \varnothing \mathrm{AC}$ induction motor drives the pump and a variable speed motor control adjusts the motor speed and hence the water flow rate. This provides a variable water velocity range from zero to the maximum flow speed at the test section. An upstream honeycomb breaks down the vortices generated in the pump and reduces the flow turbulence intensity at the test section to less than $3 \%$ for the maximum speed. Figure 4.1 sketches the frontal view of the water tunnel facility at the icing tunnel laboratory of the University of Manitoba.

\subsubsection{Test Rig and Model Turbine}

The test rig consists of a baseplate that sits on the two sides of the water tunnel upper rim and keeps the model turbine submerged at the center of the flow cross section. It also secures the instrumentation out of the water. Locating the rotor hub at the center of the flow cross section gives a clearance of one rotor diameter around the turbine to the tunnel walls and the free surface. The model turbine consists of a rotor blade secured to the turbine horizontal shaft. A right angle 1:1 bevel gearbox couples the horizontal shaft to a vertical shaft. The vertical shaft supported with ball bearings inside an upright support pipe connects the turbine shaft to the instrumentation placed on the baseplate. The turbine shaft aligns the rotor blade perpendicular to the flow direction one radius upstream of the upright support pipe. This arrangement helps minimize the influence of flow disturbances induced by the support structure on the turbine performance. Two support flat bars in a V shape secure the turbine and the upright support pipe to the baseplate. This eliminates turbine vibrations at higher flow velocities and shaft speeds. A 
connector piece fixed to the bevel gearbox flange enables replacing the shrouds around the rotor blade. The turbine shaft and the connector piece are designed to axially place the rotor blade at the smallest cross sectional area of each shroud, i.e. the inlet of the diffuser and the throat of the shroud. A schematic of the model turbine and the test rig is depicted in Figure 4.2.

\subsubsection{Rotor Blade}

The rotor blade is a $19.8 \mathrm{~cm}$ diameter three bladed $\mathrm{H} 0127$ wind turbine blade from KidWind Project Inc. The geometric characteristics of a rotor blade inspected visually and measured using a caliper with an accuracy of $0.02 \mathrm{~mm}$ are as follows: the hub diameter is $19.0 \%$ of the rotor blade diameter and the blade section consists of flat bottom airfoils. The chord, thickness, and pitch angle for selected spanwise locations on the blade are summarized in Table 4.1. An approximate $7.0 \mathrm{~mm}$ radius circular arc at $r / R=0.96$ makes a rounded end at the blade tip. The solidity of the rotor blade, the total area of blades to the rotor swept area, is 0.13 . Based on our information from the producer, the blade geometry has been optimized among several designs for an efficient wind turbine. The exact geometry of the blade cannot be disclosed as per conditions in the agreement signed with the company to receive the CAD drawing of the blade for CFD simulations. The blade is a commercial product and is available in the market for any reproduction experiment. 


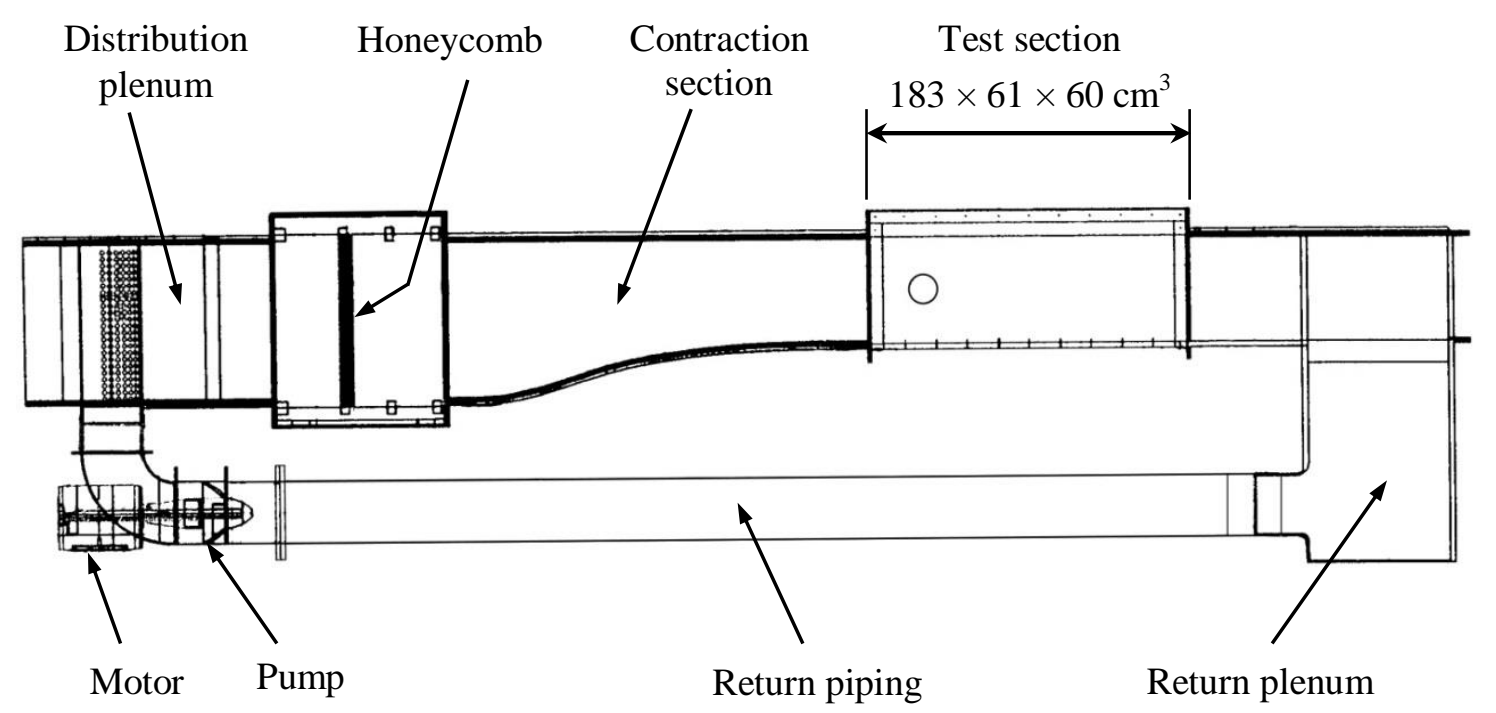

Figure 4.1: Front view of the vertical flow loop recirculating water tunnel and its components.
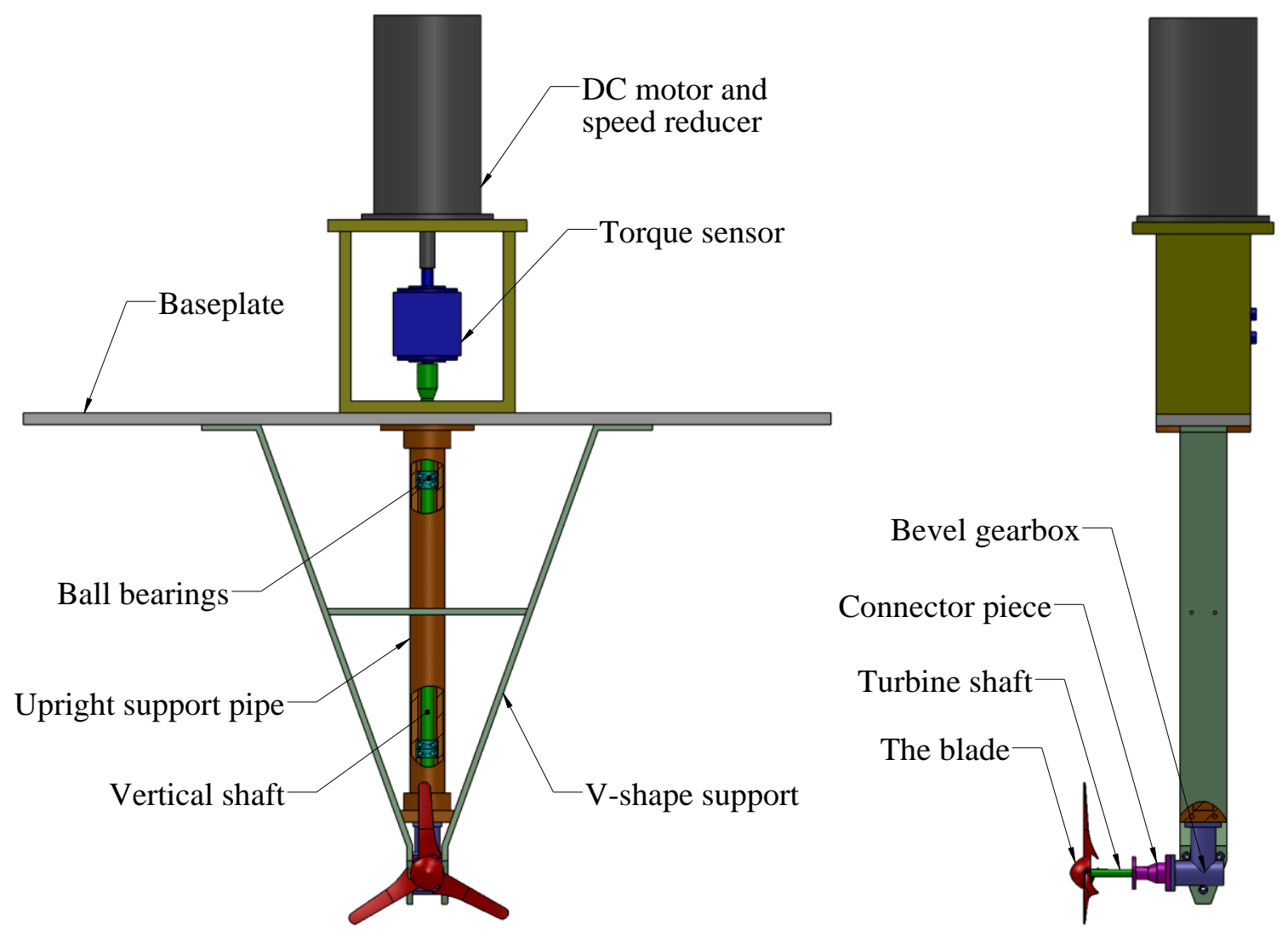

Figure 4.2: Front view (left) and side view (right) of the test rig and the model turbine without a shroud installed. The rotor blade is $19.8 \mathrm{~cm}$ in diameter. 
Table 4.1: Physical characteristics of a rotor blade at selected spanwise locations

\begin{tabular}{lccc}
\hline $\mathrm{r} / \mathrm{R}$ & $\mathrm{c} / \mathrm{R}$ & $\mathrm{t} / \mathrm{c}$ & $\operatorname{Pitch}\left({ }^{\circ}\right)$ \\
\hline 0.19 (Hub) & 0.24 & 0.14 & 30 \\
0.60 & 0.15 & 0.15 & 12 \\
0.96 & 0.12 & 0.10 & 4 \\
\hline
\end{tabular}

\subsubsection{Shroud Models}

Two shroud designs are optimized and fabricated to study and compare the effect of shroud on the performance of the model turbine. One design is a convergent-divergent duct fabricated in a rapid prototyping machine and is referred to as the shroud. The other design is a straight wall duct machined out of an acrylic block and is referred to as the diffuser. The area ratio and half angle for the shroud and the diffuser are selected based on the studies available in the literature. The schematic profiles and pictures of the shroud and the diffuser are depicted in Figure 4.3 and their physical characteristics are summarized in Table 4.2. The thickness of the diffuser wall is $9.0 \mathrm{~mm}$. 

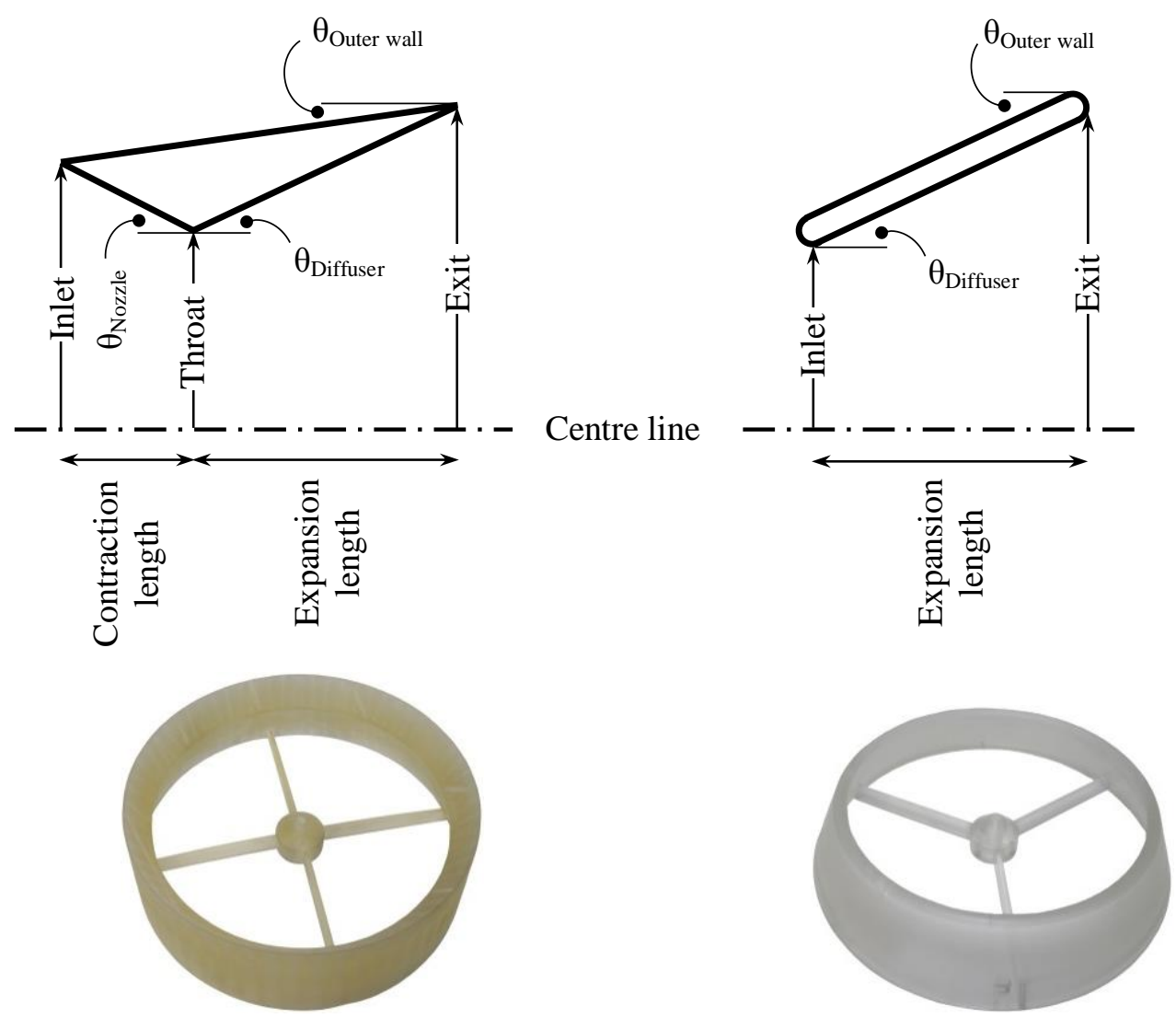

Figure 4.3: Schematic profile and picture of the shroud (left) and the diffuser (right).

Table 4.2: Dimensions of the shroud and the diffuser

\begin{tabular}{lcc}
\hline Dimension & Shroud & Diffuser \\
\hline Inlet diameter $(\mathrm{cm})$ & 22.6 & 20.1 \\
Throat diameter $(\mathrm{cm})$ & 20.1 & --- \\
Exit diameter $(\mathrm{cm})$ & 25.0 & 25.0 \\
Contraction length $(\mathrm{cm})$ & 2.5 & --- \\
Expansion length $(\mathrm{cm})$ & 5.2 & 5.2 \\
Nozzle half angle $\left({ }^{\circ}\right)$ & 27 & --- \\
Diffuser half angle $\left({ }^{\circ}\right)$ & 25 & 25 \\
Outer wall angle $\left(^{\circ}\right)$ & 8 & 25 \\
\hline
\end{tabular}




\subsection{Background of Diffuser Design}

It is now beneficial to review the diffuser design concept for diffuser augmented wind turbines before describing the method employed in designing our shrouds. A number of geometric specifications influencing the performance of a diffuser are pointed out in previous studies available in the literature. These include the exit area ratio, half angle, internal and external geometry, and the inlet shape of a diffuser. The diffuser half angle controls flow separation within the diffuser and impacts the external flow. There exists a dilemma in designing a diffuser. An aerodynamically efficient diffuser is a long diffuser with a small included angle while a practical diffuser is a compact one with a large included angle (Klein, 1981; Kline et al., 1959). The maximum half angle for a conical diffuser in order to be able to maintain a steady attached flow throughout the inner surface is $7^{\circ}$ reported in several studies (Azad, 1996; Ghose and Kline, 1978; Savkar, 1980; Strawn and Kline, 1983). Flow in such a diffuser has enough energy to overcome the viscous dissipation and adverse pressure gradient and hence stay attached to the diffuser wall (Cabuk and Modi, 1992; Chang, 1976; Gad-el-Hak and Bushnell, 1991; Lai et al., 1989; Madsen et al., 2000). For a diffuser augmented wind turbine on the other hand, a compact diffuser with a large included angle is preferred. The large exit area ratio of such a diffuser causes a larger mass flow through the turbine rotor (Fletcher, 1981). Flow separation is however inevitable in this diffuser when no rotor blade is present (McDonald and Fox, 1966). Several solutions have been proposed to avoid the flow separation in a compact large included angle diffuser. These include use of vanes or obstructions inside the diffuser or blowing an external flow to energize the diffuser internal flow. Applying suction to the boundary layer flow to keep it attached to the 
diffuser wall is also a proposed solution (Choi et al., 1998; Gersten, 1966; Tennant, 1973).

The $7^{\circ}$ half angle criterion beyond which the flow starts to separate from the inner wall of a diffuser does not apply to a diffuser enclosing a rotating turbine. No flow separation is reported inside diffuser augmented wind turbines having included angles as large as $60^{\circ}$ (Oman et al., 1977). The turbulence and the swirl created by the rotation of the rotor blade and imparted on the wake of the rotor improve the pressure recovery of the diffuser (Clausen, 1988; Kumar and Kumar, 1980; McDonald et al., 1971). This also provides an enhanced momentum transfer to the boundary layer flow on the diffuser inner wall and delays the separation (Fletcher, 1981; Foreman and Gilbert, 1979b; Lewis et al., 1977). This phenomenon is displayed well when no such improvement is seen in an experiment with a diffuser equipped with a gauze screen rather than a turbine rotor (Gilbert and Foreman, 1983). The angular momentum imparted from a rotating rotor blade on the wake flow energizes the boundary layer, stabilizes the flow, delays the onset of flow separation and therefore substantially improves the diffuser efficiency (Atligan, 1976; Gilbert and Foreman, 1979).

\subsection{Selection of Area Ratios and Half Angles}

Two wake regions develop behind the rotor blade of a hydrokinetic turbine: the near wake and the far wake. The region immediately after the rotor blade is called the near wake which influences the flow field around the rotor and the power extraction of a turbine (Vermeer et al., 2003). The fluid passing through a turbine rotor contributes its 
kinetic energy to the turbine shaft and forms a low energy wake region behind the rotor blade. The fluid in the wake does not have the sufficient energy to rapidly move downstream and hence blocks the flow passage to the rotor. This decreases the fluid mass flow rate to the turbine rotor and therefore reduces the extracted power. Another feature associated with the near wake of a hydrokinetic turbine is the tip vortices. Tip vortices are generated at the tip of the blades and move at the expanding boundary of the wake region. The fluid flowing on the pressure side of the blades tends to roll around the blades tip and go to the low pressure region at the suction side of the blades, as shown in Figure 4.4. This is known as the tip loss and is responsible for decreasing the pressure difference between the blades surfaces. It consequently decreases the lift force of the blades and effectively reduces the turbine output power. The fluid rolls generated around the blade tip then move downstream and form the tip vortices. Experimental studies on the near wake of small wind turbines have shown quantities of flow angular momentum are lost to generate tip vortices (Ebert and Wood, 2001, 1997). This angular momentum in the absence of the tip vortices could be extracted by the rotor and increases the output power. The induced velocities in the rotor plane that affect the turbine performance is another phenomenon associated with the tip vortices. Tip vortices are also known to be responsible for the rotor noise and blade vibrations (Massouh and Dobrev, 2007).

Performance of a turbine improves by controlling the negative impact of the near wake and tip vortices. A shroud enclosing the turbine rotor facilitates the expansion of the slow moving, low static pressure wake behind the rotor. A proper expansion increases the static pressure of the flow and provides sufficient kinetic energy for it to move downstream and clear the passage for the upstream flow to enter the rotor blade. This 
consequently increases the flow velocity through the rotor and increases the turbine performance (Hansen et al., 2000). Moreover, enclosing the turbine rotor in a shroud significantly reduces tip losses (Igra, 1981). A shroud with a properly designed gap between its inner wall and the blades tip prevents the fluid to roll around the blade tips and hence eliminates the tip losses. This, not only prevents the reduction of the lift force on the blades, but also prevents the formation of tip vortices. This consequently increases the turbine output power and decreases the vibration level and fatigue loads on the rotor. A thin fluid layer passing through the gap between the blades and the shroud wall also energizes the wall boundary layer flow and keeps it attached to the wall (Foreman et al., 1978; Hansen et al., 2000). The appropriate literature was consulted for selecting a proper gap size and a clearance of $0.8 \%$ of the rotor blade diameter was selected for our design (ANSI/API Standard 661, 2006).

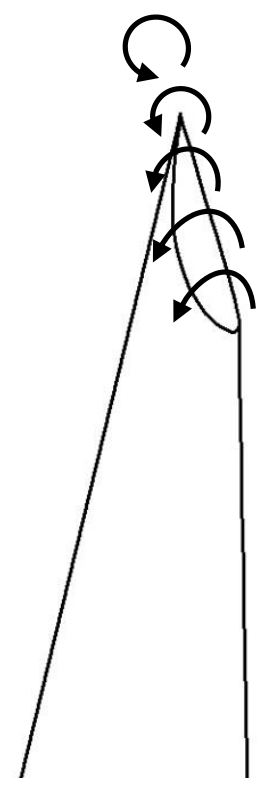

Figure 4.4: Tip vortices are generated by the fluid rolling from the pressure side to the suction side of the blades at the tip. 
The area ratio and half angle for the shroud and the diffuser are selected as follows. The exit area ratio is adopted from the CFD optimization work of Gaden (2007). Gaden optimized the diffuser half angle and the area ratio for higher power and lower drag of a turbine. A diffuser area ratio of 1.56 and half angle of $20^{\circ}$ to $30^{\circ}$ were reported as the optimum values. He further concluded the turbine power decreases outside this half angle range. The diffuser half angle was stated to be a function of the free stream velocity and was recommended to match the natural expansion of the flow through the turbine. The idea of a shroud assisting the flow in making its natural diffusion is acknowledged in a few studies in the literature (Atligan, 1976; Lewis et al., 1977). Several flow visualization experiments on wind turbine models were reviewed in order to find the natural expansion path of a flow passing a rotor blade. Alfredsson and Dahlberg (1979) as part of a research on the wake behind a solid disk documented a smoke flow visualization study performed in a wind tunnel. The smoke path shows the expansion of the flow behind the disk. The expansion angle of the flow measures approximately $25^{\circ}$ for the Reynolds number of the order $10^{5}$ based on the disk diameter. van Holten (1981) as part of a study on a general aerodynamic theory for wind energy concentrator systems compared the flows passing through a conventional rotor blade and one equipped with tip vanes. Photos of the smoke flow visualization show the expansion of the flow passing through the two rotors. The angle at which the flow expands behind the conventional rotor is $25^{\circ}$ as per our measurement. Vermeer (2001) also did smoke flow visualisation in a wind tunnel on a $20 \mathrm{~cm}$ rotor blade to study wind turbine wakes. The angle at which the flow leaves the rotor blade is $25^{\circ}$ from the photos provided. The tip vortices moving downstream are also clearly visible. Haans et al. (2005) conducted smoke flow visualization to determine tip 
vortex locations and wake expansion of a model wind turbine rotor at different blade pitch and rotor yaw angles. The experiments were carried out in a Reynolds number of the order $10^{5}$ based on the rotor diameter. Photos of the flow visualisation along with the diagrams of the tip vortex locations are provided. The results show a range of approximately $18^{\circ}$ to $31^{\circ}$ for the flow expansion angle behind the turbine rotor for different blade pitch angles in an axial flow. This is indeed in agreement with the range Gaden and Bibeau (2010) based on their numerical optimization study proposed for the diffuser half angle.

Inspecting these studies led the present author to accept $25^{\circ}$ angle as an average value for the natural expansion angle of a flow passing through a hydrokinetic turbine rotor for the range of the Reynolds number of interest. Therefore, the shroud and the diffuser were designed with $25^{\circ}$ divergence angle. A contraction segment with the half length of the expansion length was also designed for the inlet of the shroud, see Table 4.2. An inlet accelerates the flow through the rotor blade and stabilizes the entrance flow in off-axis flows (Lilley and Rainbird, 1956). It also improves uniformity and steadiness of the flow entering the shroud. A contraction section upstream of a test section in a wind or water tunnel is designed for the same purpose (Mikhail, 1979; Morel, 1977). An inlet contraction is also observed to reduce the impact of large scale turbulence on the performance of a shroud (Hussain and Ramjee, 1976). The choice of the dimensions for the inlet of the shroud resulted from the recommendations of previous studies. The inlet area ratio, ratio of the inlet to throat area, is suggested not to be larger than the exit area ratio (Lewis et al., 1977; Lilley and Rainbird, 1956). 
It is worth mentioning that two numerical optimization studies have also reported the same optimum half angle for a diffuser. Scherillo et al. (2011) in an axisymmetric CFD optimization study for a shrouded turbine found $26^{\circ}$ as the optimum angle for a diffuser. Isensee and Abdul-Razzak (2012) performed a three dimensional CFD optimization study on a diffuser augmented wind turbine and found a diffuser with $25^{\circ}$ angle the best to enhance the performance of the turbine. Interestingly the two different approaches, i.e. the empirical observation used in this study and the numerical optimization by other authors, independently resulted in the same value for the diffuser half angle. It should be noted that the two numerical works mentioned above were published after the design and fabrication of the present shroud and diffuser.

\subsection{Instrumentation}

A torque transducer and a load cell measures the torque and thrust of the turbine rotor for the entire range of power curve. A generator and variable resistance arrangement is often used to extract power from a turbine in water tunnel experiments (Myers and Bahaj, 2006). In this method it is not possible to capture the turbine power at the low tip speed ratios when the blades are stalled and the turbine produces low power. To cover the

entire span of the performance curve, we employ the method of the driving motor (Birjandi et al., 2013). A motor in this method drives the turbine at a constant speed with the torque transducer placed between the motor and the turbine, as shown in Figure 4.2. This arrangement enables measuring the rotor torque at any tip speed ratio. A 0.25 HP AC motor is used in the experiments with a 1:2 speed reducer coupled to it in order to reduce the motor $\mathrm{rpm}$ and provide the required torque. An AC motor speed 
control system governs the motor rpm and therefore the turbine rotational speed to the desired set values. A digital wave rotary torque transducer is placed between the motor and the turbine vertical shaft. It measures torque on the turbine shaft at a sampling rate of $200 \mathrm{~Hz}$ to a maximum value of $5 \mathrm{~N} \cdot \mathrm{m}$. The torque transducer has a rotational speed sensor with user adjustable range enabling direct measurements of the turbine power. The torque transducer records a negative torque when the turbine generates power and tends to spin the motor faster. A positive value shows the turbine operates at the beginning or the end of the power curve where, due to the stall or high drag of the blades, the turbine generates less power and the motor drives the turbine.

A miniature universal strain gauge load cell capable of measuring forces up to $100 \mathrm{~N}$ enables measurement of the thrust force on the turbine rotor. Needles of $0.7 \mathrm{~mm}$ diameter are used as rollers between the test rig baseplate and the tunnel rim. This along with the lubrication of the contact surfaces minimizes the surface friction and allows the rig to smoothly slide on the tunnel rim. An aluminum flat bar clamped to the water tunnel rim secures the load cell attached to the rig baseplate. A schematic of the load cell attached to the rig and the position of the rollers is depicted in Figure 4.5. In addition to torque and thrust measurements, a Nortek Acoustic Doppler Velocimetry probe measures the undisturbed flow velocity at the inlet of the water tunnel test section at a sampling rate of $200 \mathrm{~Hz}$ using $50 \mu \mathrm{m}$ diameter polyamide seeding particles. 

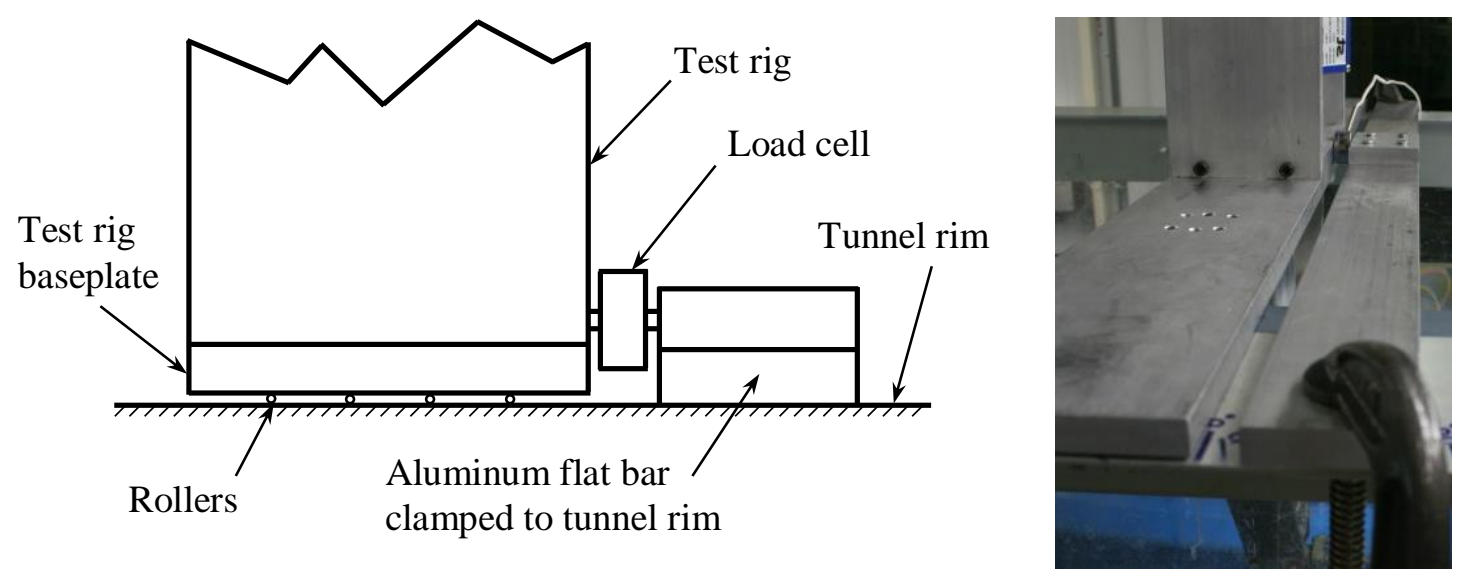

Figure 4.5: Schematic of the test rig and load cell secured between the baseplate and the clamped flat bar (left). The rollers and thoroughly lubricated surfaces allow for a smooth slide of the rig on the tunnel upper rim (right).

\subsection{Test Procedure and Data Analysis}

The measurements begin with the unshrouded model turbine in the selected water velocities of $0.7,0.9$, and $1.1 \mathrm{~m} / \mathrm{s}$. The flow velocities correspond to the Reynolds numbers of $1.38 \times 10^{5}, 1.77 \times 10^{5}$, and $2.17 \times 10^{5}$ based on the rotor diameter and the undisturbed water velocity. Turbulence intensity of the flow calculated from the ADV measurements ranges from $2.06 \%$ to $2.89 \%$. The torque exerted by the water stream on the turbine rotor is measured at each Reynolds number for the entire tip speed ratio range of the performance curve. The drag force on the turbine structure is measured at each test velocity. The thrust force on the turbine rotor at each tip speed ratio is then calculated by subtracting this drag force from the thrust acting on the whole system, i.e. the turbine and the structure (Krogstad and Adaramola, 2012). The test procedure is then repeated for the model turbine with the shroud as well as for the model turbine with the diffuser. The instruments are calibrated prior to each test following the standard procedures recommended by the manufacturers. For a given flow speed and rotor angular velocity, 
the measured torque and thrust is averaged and corrected for zero offset. Multiple tests are conducted for each configuration to ensure any variability associated with the test conditions is removed through the averaging. Then the tip speed ratio, power, torque, and thrust coefficients are respectively calculated as follows:

$$
\begin{gathered}
T S R=\frac{R \omega}{V}, \\
C_{P}=\frac{Q \omega}{1 / 2 \rho A V^{3}}, \\
C_{Q}=\frac{Q}{1 / 2 \rho A V^{2} R^{\prime}} \\
C_{T}=\frac{T}{1 / 2 \rho A V^{2}} .
\end{gathered}
$$

The torque and thrust of the model turbine are respectively represented by $\mathrm{Q}$ and $\mathrm{T}$. The Angular velocity of the turbine shaft is $\omega$ and $\mathrm{V}$ is the flow velocity. The radius and swept area of the rotor blade are represented by $\mathrm{R}$ and $\mathrm{A}$ in the equations. The coefficients are calculated based on the rotor swept area and the undisturbed water velocity. Figure 4.6 shows the model turbine with the shroud and with the diffuser in the water tunnel during an experiment.

For the off-axis experiments, the model turbine is rotated about the vertical axis passing through the apex of the rotor's hub for the range of yaw angles from $5^{\circ}$ to $25^{\circ}$ in $5^{\circ}$ increments. Yaw angle is the angle between the turbine axis and the flow direction. Rotating the turbine about the specific axis discussed keeps the turbine at the centre of the test section. Figure 4.7 shows the model turbine in a yaw experiment and the angle signs generated on a full scale drawing of the tunnel and marked on the tunnel rims. 

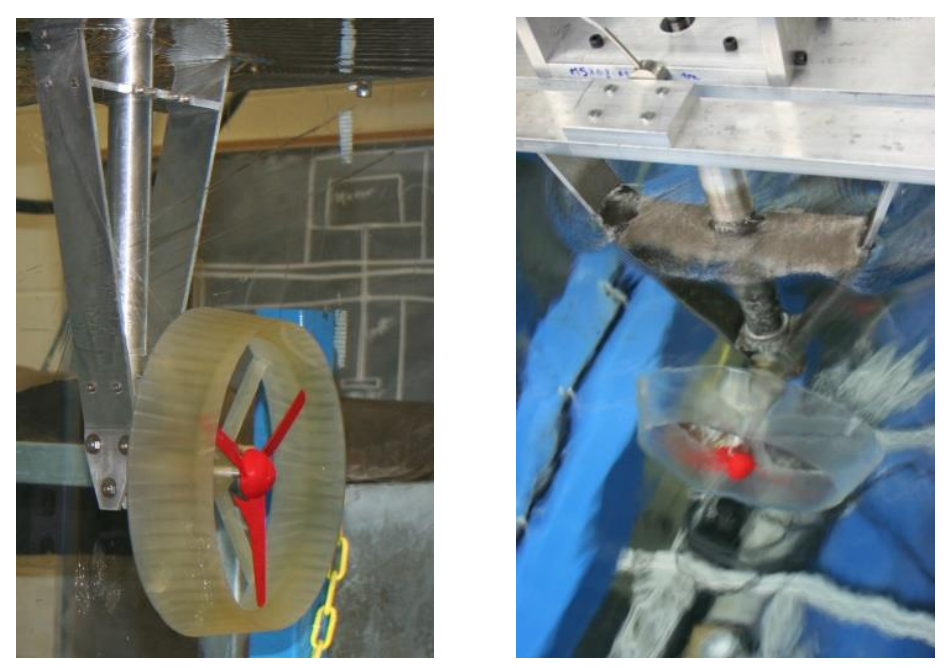

Figure 4.6: The model turbine with the shroud (left) and top view of the turbine with the diffuser (right) in the water tunnel during an experiment.

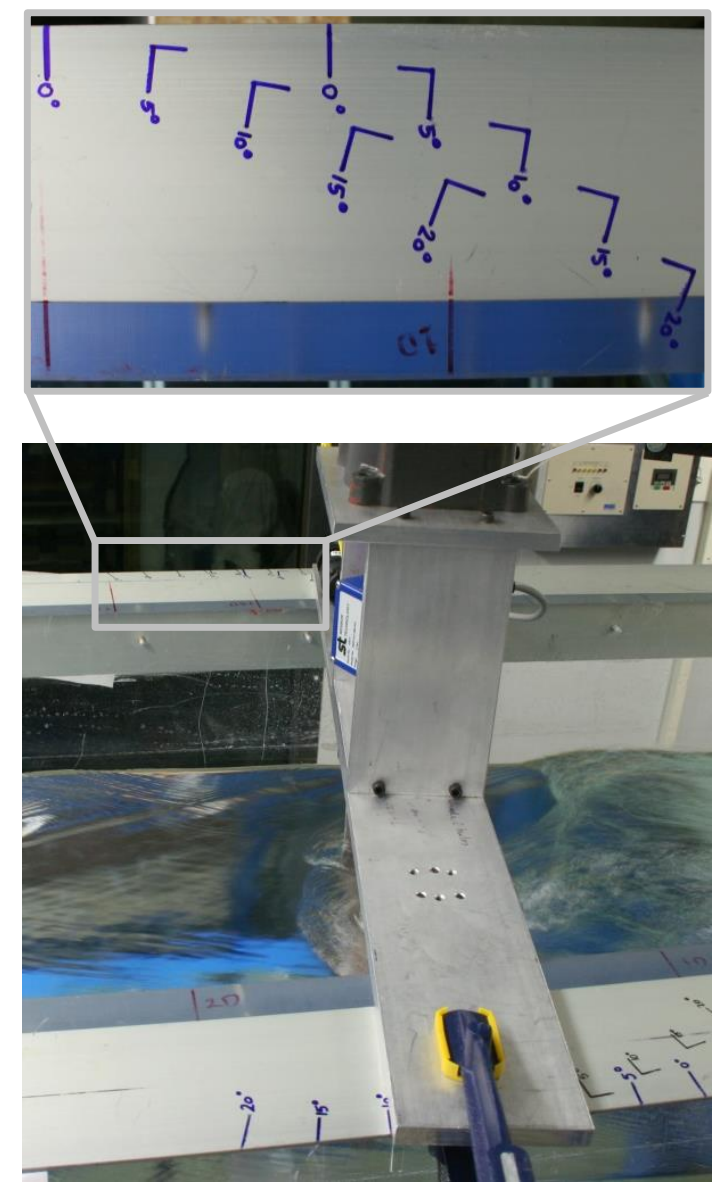

Figure 4.7: The model turbine operating at $10^{\circ}$ yaw angle in a yaw misalignment experiment. The $25^{\circ}$ angle sign was marked later and does not appear in the photo. 


\subsection{Blockage Correction}

The blockage caused by a model turbine in a wind tunnel has been the subject of studies by wind tunnel experimentalists. Different levels of influence on experimental results are reported in the literature based on the nature of the results sought, i.e. the wake expansion or the turbine performance. Ross and Altman (2011) reviewed the different schemes employed in the literature to correct the blockage effect on wind turbine experiments. Ross (2010) conducted experiments on a vertical axis wind turbine in two different wind tunnels. He concluded large wall interaction and wake propagation could occur at a smaller blockage ratio than what is generally accepted in the literature as a standard blockage. There are researchers on the other hand who suggest an upper limit of about $10 \%$ for the blockage ratio in order to avoid the wind tunnel wall interference on the measurements (McTavish et al., 2012; Wilson, 2009). Chen and Liou (2011) conducted wind tunnel experiments to study the effect of tunnel blockage on the power coefficients of horizontal axis wind turbines. They concluded the blockage correction is less than 5\% for a $10 \%$ blockage ratio. This, they believe, confirms the accepted general rule in the literature that no correction is necessary for blockage ratios less than $10 \%$.

Placing a turbine in a water tunnel partially blocks the flow passage in the tunnel test section. This increases the flow velocity upstream of the turbine and results in the increased values for the measured torque and thrust. Therefore, the turbine performance obtained in a partially blocked flow needs correction in order to represent the turbine performance in an unbounded free stream. The velocity correction used by Bahaj et al. (2007) along with the model proposed by Whelan et al. (2009) is employed to correct the 
free surface proximity and blockage effects. Bahaj et al. employed the method of Barnsley and Wellicome (1990) who modified equations of wake expansion for a wind turbine (Glauert, 1947). The method is based on an actuator disk model where the flow is assumed inviscid and uniform across the cross sections of the streamtube enclosing the turbine disk. A uniform thrust force is assumed on the disk as a result of the pressure drop across it. The model leads to an equivalent open water or free stream velocity $V_{F}$ as follows:

$$
\frac{V}{V_{F}}=\frac{\beta}{\beta^{2}+C_{T}^{T} / 4},
$$

where $C_{T}^{T}$ is the thrust coefficient calculated from water tunnel measurements and $\beta$ is the ratio of the flow velocity across the disk to the tunnel velocity, V. Bahaj et al. found $\beta$ in an iterative manner using two equations derived from the conservation of mass and momentum principles applied to a wall bounded flow over the actuator disk. Equations used to find $\beta$ relate the blockage ratio, $\mathrm{B}$, the ratio of the bypass flow to the tunnel velocity, $\tau$, and the ratio of the flow velocity far downstream of the disk to the tunnel velocity, $\alpha$. Blockage ratio is the ratio of the disk area to the flow cross sectional area and bypass flow is the portion of the flow outside the streamtube containing the turbine disk. Bahaj et al. then used the velocity ratio, Equation (4.5), to correct the power and thrust coefficients as well as the tip speed ratio as follows:

$$
\begin{aligned}
& C_{P}=C_{P}^{T}\left(\frac{V}{V_{F}}\right)^{3}, \\
& C_{T}=C_{T}^{T}\left(\frac{V}{V_{F}}\right)^{2},
\end{aligned}
$$




$$
T S R=T S R^{T}\left(\frac{V}{V_{F}}\right) .
$$

Where $C_{P}^{T}$ and $T S R^{T}$ are the values calculated from water tunnel measurements. The correction equation for the torque coefficient would be the same as the thrust coefficient as they both are functions of the velocity squared. In this work, we obtain $\beta$ from the model proposed by Whelan et al. (2009) which accounts for the free surface proximity as well as wall the blockage effect. Whelan et al. extended the one-dimensional actuator disk analysis of Garrett and Cummins (2007) who considered the turbine flows constrained between two rigid surfaces to allow for deformation of the free surface. Deformation of the free surface is due to the pressure change in the region of the turbine and is associated with the Froude number of the flow. Combining the continuity, Bernoulli, and momentum equations Whelan et al. derived a quartic equation in terms of the bypass flow velocity ratio $\tau$ as follows:

$$
\begin{gathered}
F r^{2} \tau^{4}+4 \alpha F r^{2} \tau^{3}+\left(4 B-4-2 F r^{2}\right) \tau^{2}+\left(8-8 \alpha-4 F r^{2} \alpha\right) \tau \\
+\left(8 \alpha-4+F r^{2}-4 \alpha^{2} B\right)=0
\end{gathered}
$$

in which $F r=V / \sqrt{g H}$ is the upstream Froude number with $\mathrm{g}$ the gravity and $\mathrm{H}$ the upstream water level. This equation gives real solutions of $\tau$ for certain range of Fr, B, and $\alpha$ for which the bypass flow remains subcritical. Obtaining $\tau$ and $\alpha$ from the solution of Equation (4.9), the thrust coefficient is then calculated as:

$$
C_{T}=\tau^{2}-\alpha^{2},
$$

and the ratio of the flow velocity across the disk to the tunnel velocity is given by: 


$$
\beta=\frac{\alpha}{B(\tau-\alpha)}\left[\tau\left(1-\frac{F r^{2}}{2}\left(\tau^{2}-1\right)\right)-1\right] .
$$

The model is applicable to any device causing a pressure drop in the flow direction. Therefore, we also apply it to the shrouded model turbines provided that the blockage ratio is calculated based on the maximum cross sectional area of the shroud and the diffuser, i.e. their exit area. Equation (4.9) is solved for the Froude numbers and blockage ratios associated with the experiments and values of $\mathrm{C}_{\mathrm{T}}$ and $\beta$ are then calculated from Equations (4.10) and (4.11). The Froude numbers associated with the 0.7, 0.9, and $1.1 \mathrm{~m} / \mathrm{s}$ flow velocities are respectively $0.29,0.37$, and 0.45 . Having found the values of $\beta$ at any corresponding experimental thrust coefficient, Equation (4.5) is used to find the equivalent open water velocity. The power and thrust coefficients as well as the tip speed ratio are then corrected using Equations (4.6) to (4.8). As an example, a typical thrust coefficient of 0.86 for the model turbine with the shroud at the water velocity of $0.9 \mathrm{~m} / \mathrm{s}$ results in $2.8 \%$ reduction in the tip speed ratio, $5.5 \%$ reduction in the thrust coefficient, and $8.1 \%$ reduction in the power coefficient.

In reviewing the literature of diffuser augmented wind turbines in Chapter 2 an increase in the performance of shrouded turbines is reported in yaw operations. The increased blockage ratio of a shrouded turbine with a large length to diameter ratio in yaw operations is responsible for that factitious higher performance. Due to the small length to diameter ratio of the shroud and the diffuser in our experiments, see Table 4.2 , the blockage ratio decreases with increasing the yaw angle and no such an artificial elevated power is observed. 


\subsection{Uncertainty of Measurements}

To calculate the uncertainty of measurements, individual uncertainties associated with the experiments are estimated and combined to give an overall quantity. There are two approaches in estimating uncertainties: Type A and Type B evaluations (Bell, 1999). Type A evaluation is calculated from a dataset with repeated readings while Type B is usually calculated from the measurement uncertainties of an instrument found in the calibration certificates. Uncertainties should be at the same confidence level before they can be combined. This is done by expressing the uncertainties in the form of the standard uncertainty. Standard uncertainty, u, is a margin with a size of one standard deviation on either side of the average value of a set of repeated readings. After computing the estimated standard deviation, s, Type A uncertainty is calculated for the set of repeated readings as follows:

$$
u_{A}=\frac{s}{\sqrt{n}}
$$

where $\mathrm{n}$ is the number of data points in the set. Type A evaluation which is the standard uncertainty of mean is also called the standard deviation of mean or the standard error of mean. Type B uncertainty for a normal distribution is calculated as follows:

$$
u_{B}=\frac{a}{2}
$$

in which a is the semi-range or the half-width between the upper and lower limits of the uncertainty of the measuring instrument. The quantity 2 appearing in the denominator is

for normally distributed uncertainties and would be replaced by $\sqrt{3}$ for a rectangular or a 
uniform distribution. The uncertainty reported in the calibration certificate of an instrument is usually assumed to be normally distributed (Dietrich, 1991). The combined standard uncertainty is then calculated from Type A and Type B standard uncertainties using the "summation in quadrature" or "root sum of the squares" rule:

$$
u_{c}=\sqrt{u_{A}^{2}+u_{B}^{2}}
$$

When a quantity, $\mathrm{C}_{\mathrm{P}}$ for instance, is a combination of different quantities, see Equation (4.2), the relative combined uncertainty is calculated as follows:

$$
\frac{u_{c}\left(C_{P}\right)}{C_{P}}=\sqrt{\left[\frac{u_{c}(Q)}{Q}\right]^{2}+\left[\frac{u_{c}(\omega)}{\omega}\right]^{2}+\left[2 \frac{u_{c}(R)}{R}\right]^{2}+\left[3 \frac{u_{c}(V)}{V}\right]^{2}} .
$$

The combined standard uncertainty is considered equivalent to one standard deviation having a confidence level of approximately $68 \%$. The expanded uncertainty, U, can be computed for higher levels of confidence by multiplying the combined standard uncertainty by a coverage factor, $\mathrm{k}$ :

$$
U=k u_{c}
$$

A coverage factor of 2 provides the most commonly used confidence level of $95 \%$ for the expanded uncertainty. Coverage factors of 2.58 and 3 give confidence levels of $99 \%$ and $99.7 \%$, respectively. This is for a normally distributed combined standard uncertainty and different coverage factors are used for other less common shapes of distribution (Chatfield, 1983). 
Accuracy of the torque transducer and the load cell in our experiments, reported in the instrument specifications is $\pm 0.25 \%$ of the full scale. The ADV measures the flow velocity with an accuracy of $\pm 0.5 \%$ of the measured velocity $\pm 1 \mathrm{~mm} / \mathrm{s}$. The accuracy in the length measurements is $\pm 0.02 \mathrm{~mm}$. The expanded uncertainty for the power and thrust coefficients are calculated based on Type A and Type B standard uncertainty evaluations in accordance to JCGM (2008). The uncertainty of measurements for typical values of $C_{P}=0.70$ and $C_{T}=0.78$ for the model turbine with the diffuser at the water velocity of $1.1 \mathrm{~m} / \mathrm{s}$ are as follows. The relative combined standard uncertainty is calculated to be $2.15 \%$ and $1.28 \%$ with the confidence level of approximately $68 \%$ for the respective coefficients. Therefore, the power and thrust coefficients are stated as $C_{P}=0.70 \pm 0.03$ and $C_{T}=0.78 \pm 0.02$. The reported expanded uncertainty is based on the standard uncertainty multiplied by a coverage factor of 2 providing a level of confidence of approximately $95 \%$. For the purpose of clarity, error bars are only added to selected figures. 


\section{Chapter 5 Performance in Inline Operation}

\subsection{Foreword}

In the introduction of hydrokinetic turbines in Chapter 1, the low power density was mentioned as a challenge for hydrokinetic energy. Diffuser augmented wind turbines have been proposed and investigated as a remedy in the counterpart wind industry for more than fifty years (van Bussel, 2007). Phillips (2003) reviewed the theoretical, experimental, and numerical studies done to the date for making the idea commercially viable. A successful commercial unit has not been introduced in the market due to the high loads exerted on the duct, heavy support structure needed, and expensive yawing mechanisms (Yuce and Muratoglu, 2015). This added complexity along with its high cost compared to the simple method of extending the rotor diameter is the main reason preventing the commercial use of the idea (Shives and Crawford, 2011). Continuous technological achievements in mechanical engineering, material science and manufacturing techniques enable construction of larger size blades. This superseded the diffuser augmentation in wind turbine industry, as there is relatively no space limitation for installing larger turbines in the atmosphere. Currently $126 \mathrm{~m}$ diameter wind turbines with 7.5 MW rated power are in service and plans are to make $250 \mathrm{~m}$ diameter turbines with $20 \mathrm{MW}$ rated power (Fichaux et al., 2011). Nevertheless, the idea of diffuser 
augmented wind turbine is not yet disappeared and is still the subject of some studies, however, confined to academia. Takahashi et al. (2012), Isensee and Abdul-Razzak (2012), van Dorst (2011), ten Hoopen (2009), Ohya et al. (2008), Abe et al. (2006), and Mertens (2006) to mention a few, have recently done numerical and experimental studies on diffuser augmented wind turbines. Due to some beneficial aspects available for hydrokinetic turbines (see Chapter 1) and in the absence of a reliable and conclusive research, there have been tendencies to employ shrouds in hydrokinetic turbine technology. Companies interested in producing and employing shrouded hydrokinetic turbines along with their prototype turbines are reviewed in Chapter 2.

Performance characteristics of the shrouded and unshrouded model turbine in inline operation are presented in this chapter. The experimentally obtained reliable and consistent power, torque, and thrust coefficient curves are aimed to quantify the effect of shroud on the performance of a hydrokinetic turbine. Discussions continue to find the size of an extended blade that can generate the same power as the shrouded turbine to put the efficiency gains into a proper perspective. Some advantages and disadvantages of a shrouded turbine versus extending the rotor blade are also presented to provide an insight into the study of shrouded hydrokinetic turbines.

\subsection{Results and Discussion}

Figures 5.1 to 5.10 show the power, torque, and thrust coefficient curves of the model turbine obtained in the water tunnel experiments at the three Reynolds numbers investigated. The coefficients are calculated based on the blade swept area and are 
corrected for free surface proximity and blockage effects. The results are for the unshrouded turbine, turbine with the shroud, and turbine with the diffuser. The three configurations are respectively referred to as blade, shroud, and diffuser in the diagrams. The experimental data for the shrouded and unshrouded model turbine is available in (Shahsavarifard and Bibeau, 2014a). The results available in this chapter are published as a conference paper as well as a journal paper (Shahsavarifard et al., 2015, 2013).

\subsection{Power Coefficients}

Figures 5.1 to 5.3 depict the performance curves for the shrouded and unshrouded model turbines at $0.7,0.9$, and $1.1 \mathrm{~m} / \mathrm{s}$ water velocities. The curves consistently start at zero, reach a maximum at a tip speed ratio of approximately 4 , and descend at a tip speed ratio of approximately 7 for the three configurations. The optimum tip speed ratio of the turbine is observed to be approximately at the middle of the performance curve. The same consistent trend is also observed for the shrouded turbine where the shroud and the diffuser enhance the performance of the turbine. It is observed in the three diagrams that the shroud increases the turbine power to some extent while the diffuser is more effective. The maximum power coefficient for the unshrouded turbine, the blade, is 0.33 , 0.38 , and 0.40 at $0.7,0.9$, and $1.1 \mathrm{~m} / \mathrm{s}$ water velocities, respectively. The maximum power coefficient for the model turbine with the shroud increases to $0.50,0.54$, and 0.55 at the respective water velocities. The maximum performance coefficient of the model turbine with the diffuser is $0.63,0.71$, and, 0.70 at the respective water velocities of the experiments. This is a significant power enhancement for the diffuser considering its compact design and small length to diameter ratio compared to all previous designs in the 
literature. This confirms the accuracy of the design procedure employed. Placing a shroud on the turbine pushes the peak of the performance curve to the higher tip speed ratios. The optimum performance of the shrouded turbine is observed to occur at a higher tip speed ratio than the maximum performance of the unshrouded turbine. This behaviour has been seen and reported by other authors as well (Phillips et al., 1999). A benefit of a higher optimum tip speed ratio is the reduction of the cut-in speed of the turbine. We can see from the diagrams that the shift in the optimum tip speed ratio is larger when the power enhancement is more. The maximum power coefficient for the turbine with the diffuser occurs at a higher tip speed ratio than that of the turbine with the shroud. Similarly, the maximum performance of the turbine with the shroud has a higher tip speed ratio than that of the unshrouded turbine. Operating at a higher tip speed ratio translates to a higher angular velocity of the turbine shaft when the flow velocity is constant. The desired power of a turbine can be generated at a lower torque when the turbine shaft spins at a higher rotational speed. This results in a lighter turbine shaft and a longer gearbox life (Ahmed, 2012). A higher angular velocity is also an advantage for the turbine generator (Shives and Crawford, 2011). In addition, a high speed low torque turbine experiences less power losses due to the wake rotation than a low speed high torque turbine (Hau and von Renouard, 2013). A higher tip speed ratio however, increases hub forces and creates a higher noise level that could become a concern for a wind turbine but not for a hydrokinetic turbine (Ahmed, 2012).

Three regimes of operation are distinguished in a performance curve: the low tip speed ratio, the optimum tip speed ratio, and the high tip speed ratio regimes. The high tip speed ratio portion starts from runaway point and ends around the peak area of the curve. 
Runaway point is the high tip speed ratio end of a performance curve where the turbine does not generate power. The increased drag force on the blades is responsible for the low turbine power in this region where the low angles of attack at high tip speed ratios reduces the lift to drag ratio of the blades. Decreasing the tip speed ratio increases the angles of attack and consequently the lift to drag ratio of the blades. The maximum power coefficient of the turbine occurs at the optimum tip speed ratio where the blades generate the maximum lift due to the appropriate angles of attack (Burton et al., 2001). Passing the optimum tip speed ratio portion of the curve by further decreasing the tip speed ratio, results in the more increase in the blades angles of attack. The first indications of stall, at the low tip speed ratio portion of the curve, occur at a location close to the blades root due to the high angles of attack. The flow separation spreads from the root to the body of the blades at lower tip speed ratios and higher angles of attack where the blades move in to the deep stall mode (Corten, 2001). This significant drop in the lift force of the blades is responsible for the reduced power of the turbine at the low tip speed ratio portion of the performance curve. 


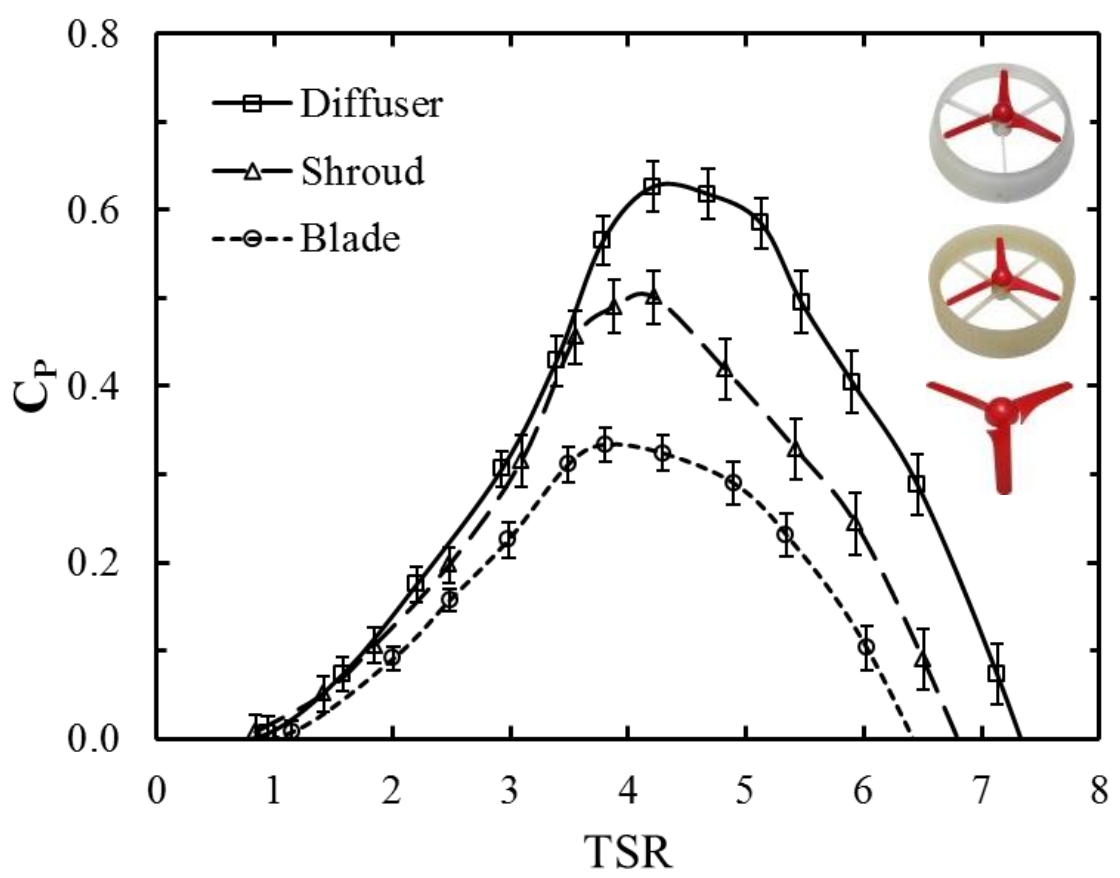

Figure 5.1: Performance coefficient curves of the model turbine at $0.7 \mathrm{~m} / \mathrm{s}$ water velocity.

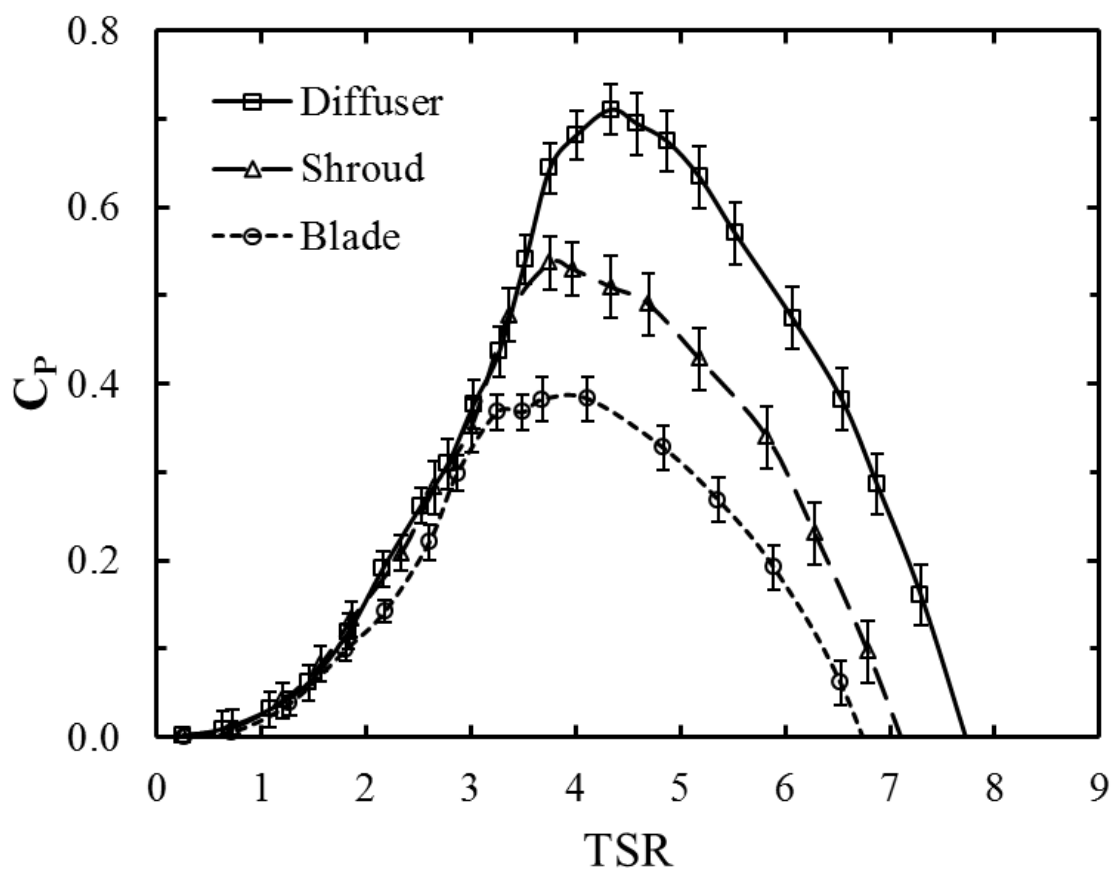

Figure 5.2: Performance coefficient curves of the model turbine at $0.9 \mathrm{~m} / \mathrm{s}$ water velocity. 


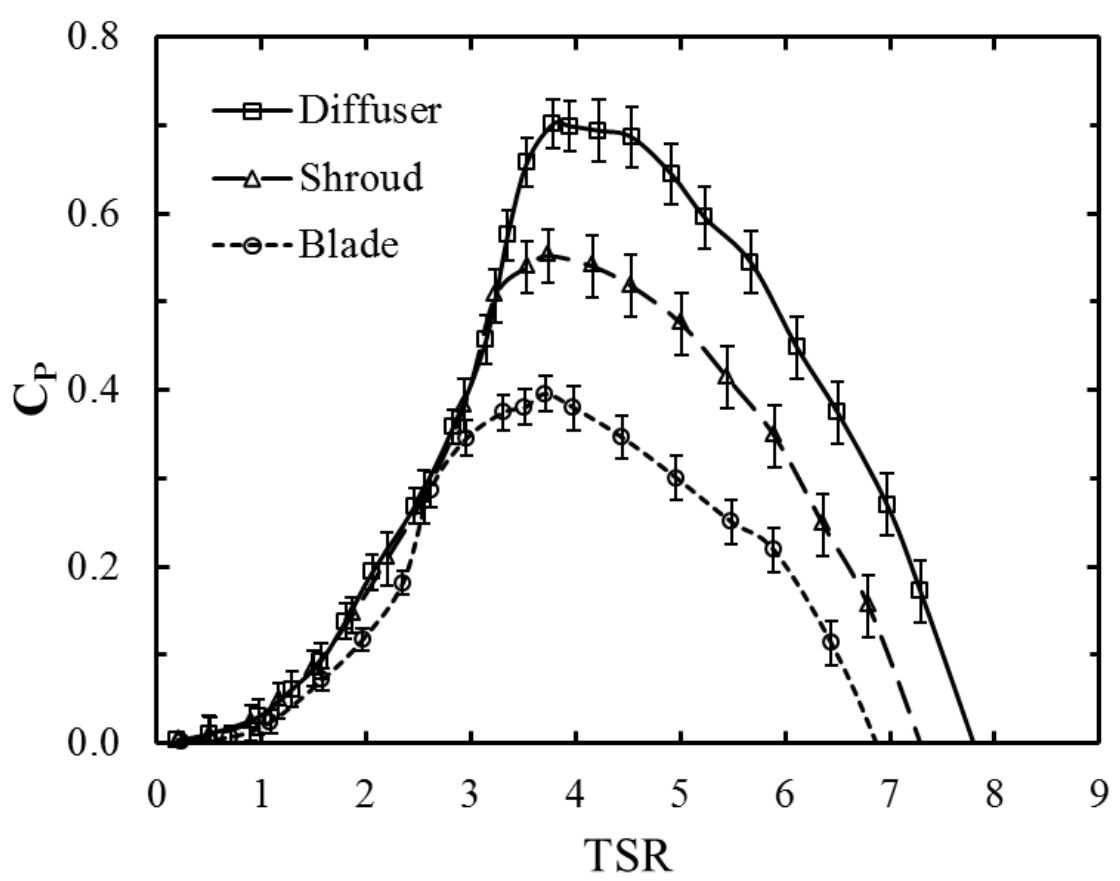

Figure 5.3: Performance coefficient curves of the model turbine at $1.1 \mathrm{~m} / \mathrm{s}$ water velocity.

It is seen from Figures 5.1 to 5.3 that the low tip speed ratio portion of the performance curves for the shrouded and unshrouded turbines overlap. This means the shroud and the diffuser are unable to enhance the turbine performance in this region. The reason could be explained by realizing the role of a shroud in increasing the flow velocity through the rotor plane as explained in section 4.4. The increase in the flow velocity increases the angles of attack at the blades and consequently intensifies the stall condition and therefore no power enhancement occurs. Increasing the flow velocity and the angles of attack at the high tip speed ratio portion reduces the drag force on the blades and enhances the performance. The shroud and the diffuser have equal diffuser half angles and expansion lengths. The better performance of the diffuser over the shroud despite of the shroud having an inlet can be explained by its three times larger outer wall angle. Wind tunnel experiments on diffusers have shown the impact of the flow over the 
external wall on the internal flow. A larger angle of the external wall accelerates the flow and creates low static pressure at the exit which causes more flow to be drawn into the turbine (de Vries, 1979; Foreman and Gilbert, 1983; Loeffler Jr. and Vanderbilt, 1978). Phillips (2003) in a wind tunnel experiment increased the angle of the external wall of a diffuser augmented wind turbine and obtained additional performance enhancement. $\mathrm{He}$ further concluded the best shape for the exit of a shroud is a radial flap, an idea Shives and Crawford (2011) also found in their numerical study. A flange shaped shroud has been investigated in several studies (Abe et al., 2005; Ohya and Karasudani, 2010). A separation region with strong vortices occurs behind the brim which creates a low pressure region at the shroud's exit as shown in Figure 5.4. This low pressure region draws more flow into the duct and accelerates the flow over the rotor (Ohya et al., 2008; Setoguchi et al., 2004). Such a shroud creates a large drag force on the turbine that increases the mooring and anchor loads.

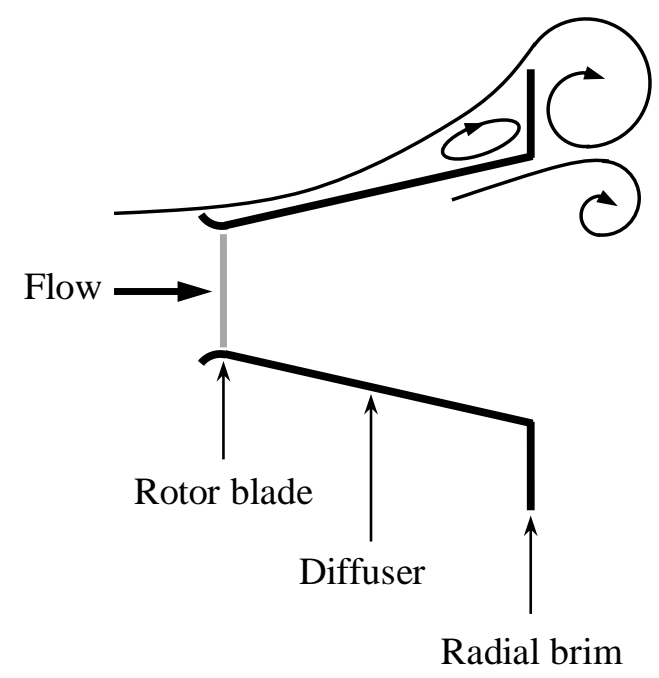

Figure 5.4: A flange shaped diffuser with strong vortices behind the radial brim. 


\subsection{Torque Coefficients}

Figures 5.5 to 5.7 depict the experimental torque coefficient curves for the shrouded and unshrouded model turbines at $0.7,0.9$, and $1.1 \mathrm{~m} / \mathrm{s}$ water velocities. The torque coefficients are obtained by dividing the power coefficients by the tip speed ratio and is useful in evaluating the self-starting capability of turbines (Jamieson, 2011). The principal use of torque curves is for the torque assessment of a gearbox and generator coupled to a turbine shaft. Low torque is desirable to reduce the gearbox costs. The optimum tip speed ratio operating point for power and torque curves are not the same. The peak of a torque curve occurs at a lower tip speed ratio than the peak of a power curve for a hydrokinetic turbine (Burton et al., 2001).

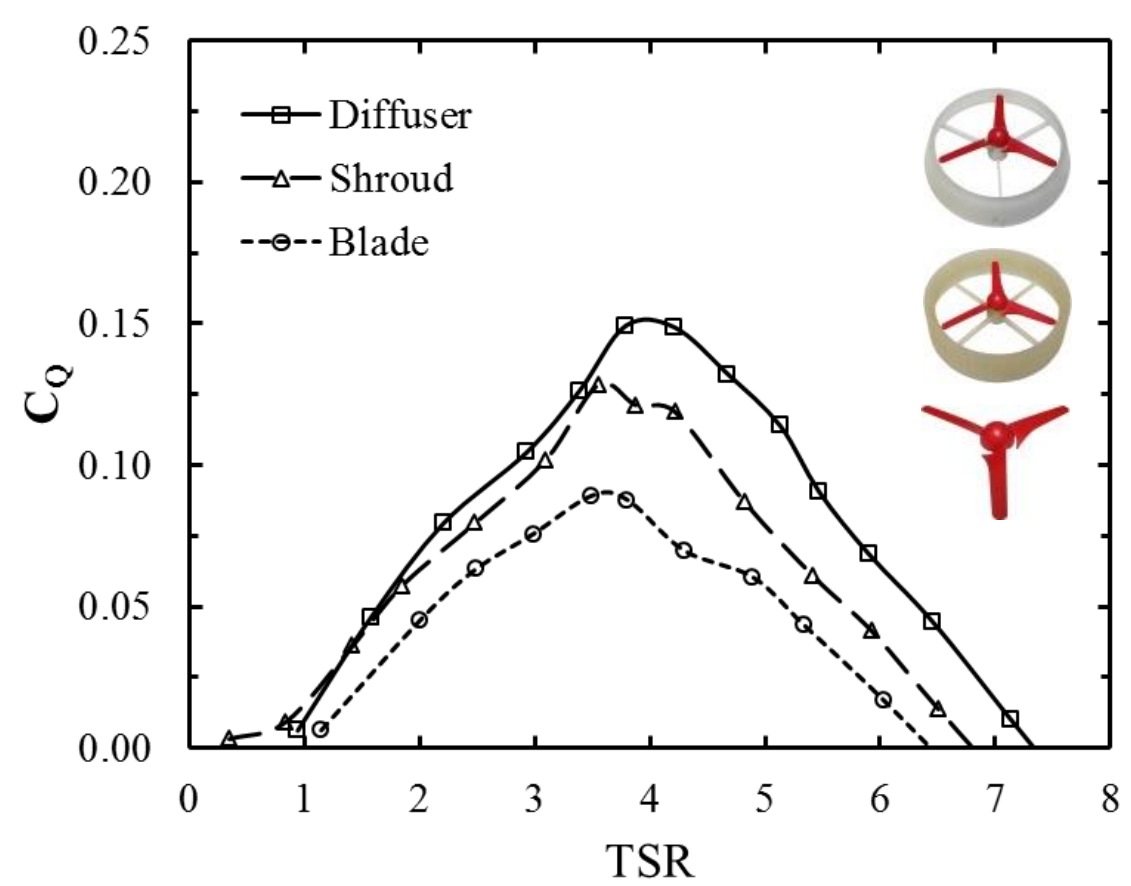

Figure 5.5: Torque coefficient curves of the model turbine at $0.7 \mathrm{~m} / \mathrm{s}$ water velocity. 


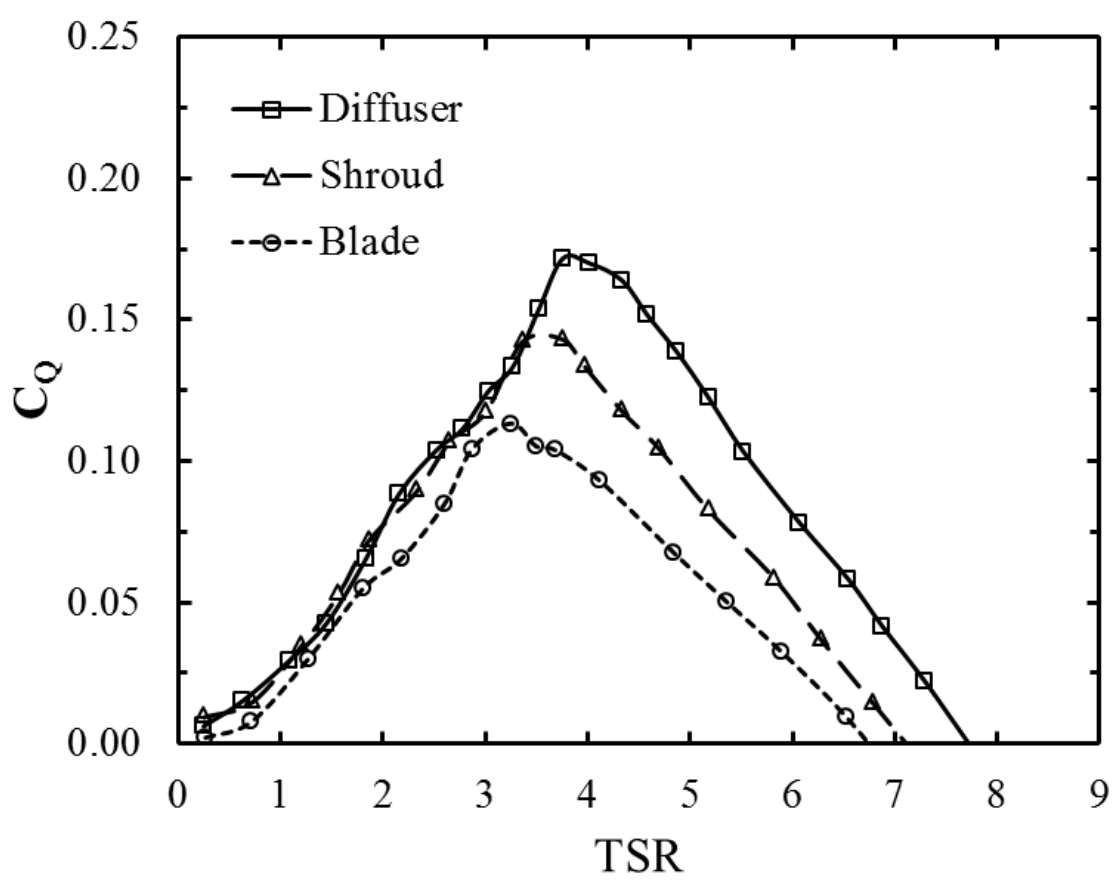

Figure 5.6: Torque coefficient curves of the model turbine at $0.9 \mathrm{~m} / \mathrm{s}$ water velocity.

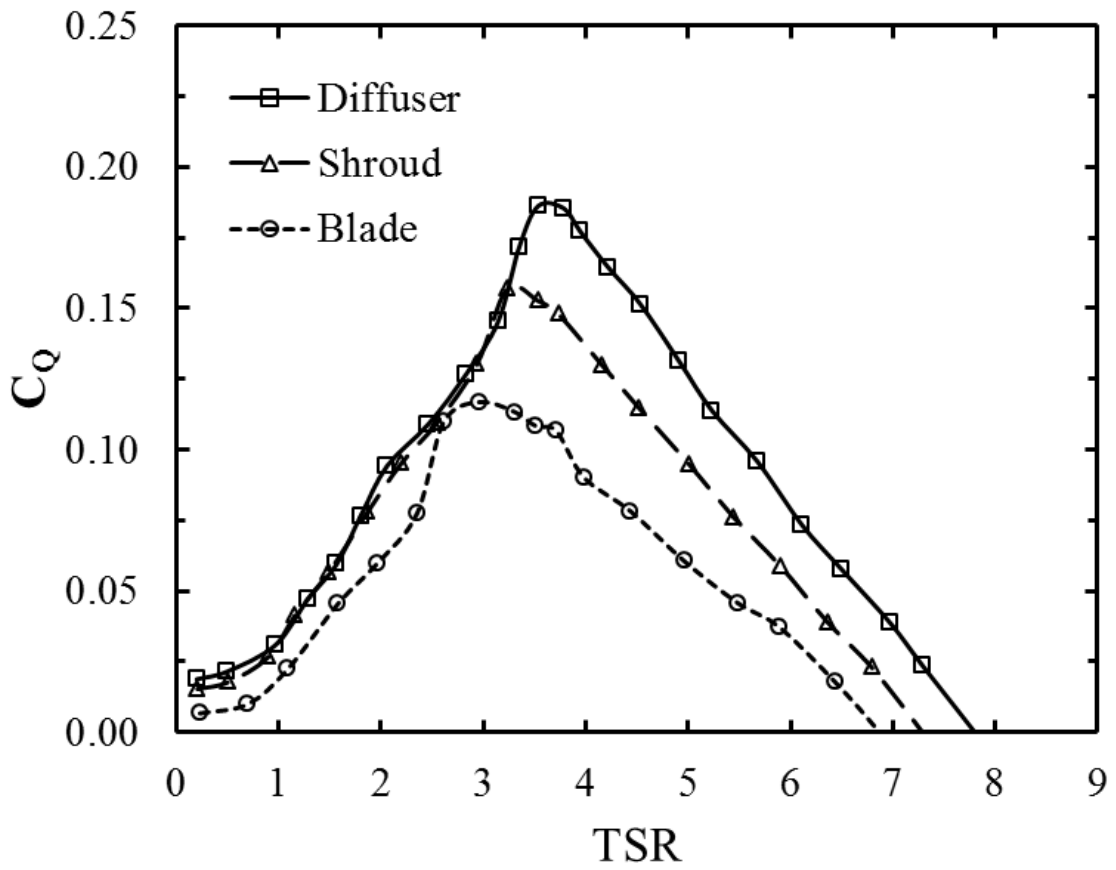

Figure 5.7: Torque coefficient curves of the model turbine at $1.1 \mathrm{~m} / \mathrm{s}$ water velocity. 


\subsection{Thrust Coefficients}

Trust force on a turbine rotor is directly transferred to the turbine structure and affects the design of the structure and anchoring systems (Burton et al., 2001). It is important to have an exact estimation of the thrust force on a turbine as underestimating the thrust force could result in failure in large scale deployments (Shahsavarifard et al., 2014a, 2014b). Figures 5.8 to 5.10 are the thrust coefficient curves for the shrouded and unshrouded model turbine at $0.7,0.9$, and $1.1 \mathrm{~m} / \mathrm{s}$ water velocities of the experiments. The results demonstrate a consistent increasing trend where the thrust force starts from a minimum value at zero tip speed ratio when the turbine is stopped. This is because the turbine has the least frontal area when the rotor blade is at rest and therefore has the minimum thrust force. The trust force then continuously increases as tip speed ratio increases (Tong, 2010). The unshrouded turbine, the blade, has the least frontal area compared to the turbine with the shroud and the turbine with the diffuser. This explains the lower drag force of the unshrouded turbine as it creates the least blockade against the water flow. Increasing the rotor angular velocity makes the rotor blade more impermeable to the flow resulting in the higher thrust coefficients as tip speed ratio increases. Thrust coefficients of a turbine rotor can reach values as high as the drag coefficient of a circular disk, 1.17 (Fox et al., 2004). Thrust coefficients in sufficiently high tip speed ratios can reach as high as 2.0 for heavily loaded rotors (Manwell et al., 2009). The experiments resulted in compatible thrust and power coefficients for the model turbine. The unshrouded turbine with the least thrust force has the lowest performance and the shrouded model turbines having higher thrusts achieve higher power coefficients. It is observed that the power curve has an ascending-descending behaviour 
with a maximum at an optimum tip speed ratio whereas the thrust force has a rising behaviour. The optimum power occurs where the product of the thrust force and the local velocity at the rotor plane is the maximum (see Section 3.1). Although the thrust force continues to increase with increasing tip speed ratio, the flow velocity at the rotor disk decreases due to the increased flow blockage at higher rotor speeds. Therefore, the maximum power and the maximum thrust do not share the same tip speed ratio (Manwell et al., 2009).

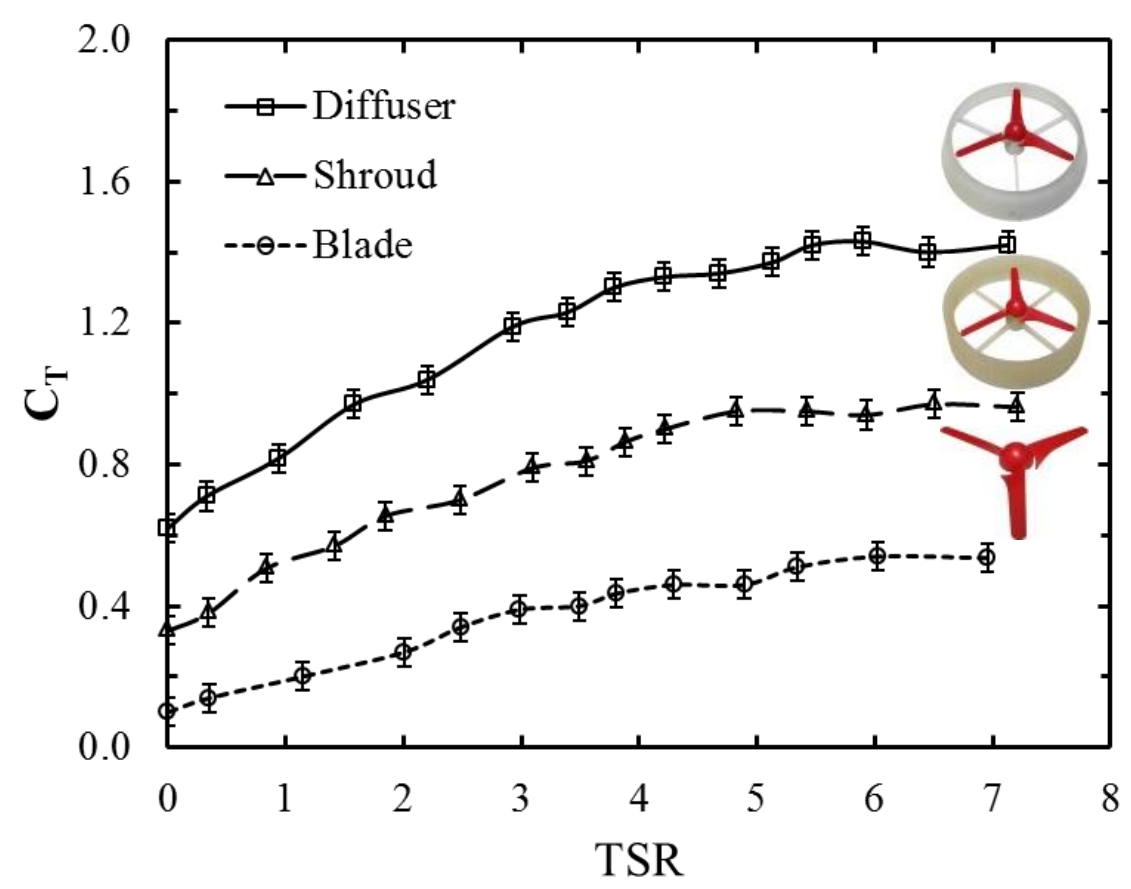

Figure 5.8: Thrust coefficient curves of the model turbine at $0.7 \mathrm{~m} / \mathrm{s}$ water velocity. 


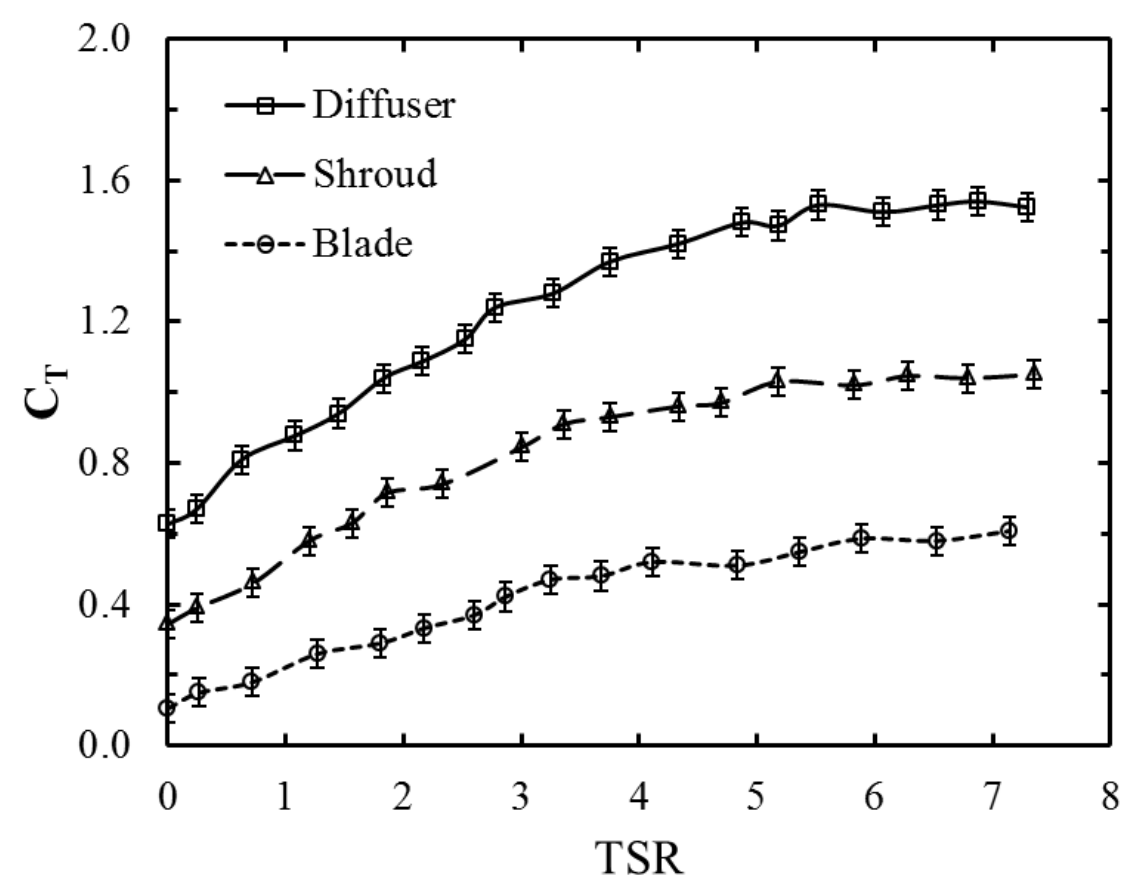

Figure 5.9: Thrust coefficient curves of the model turbine at $0.9 \mathrm{~m} / \mathrm{s}$ water velocity.

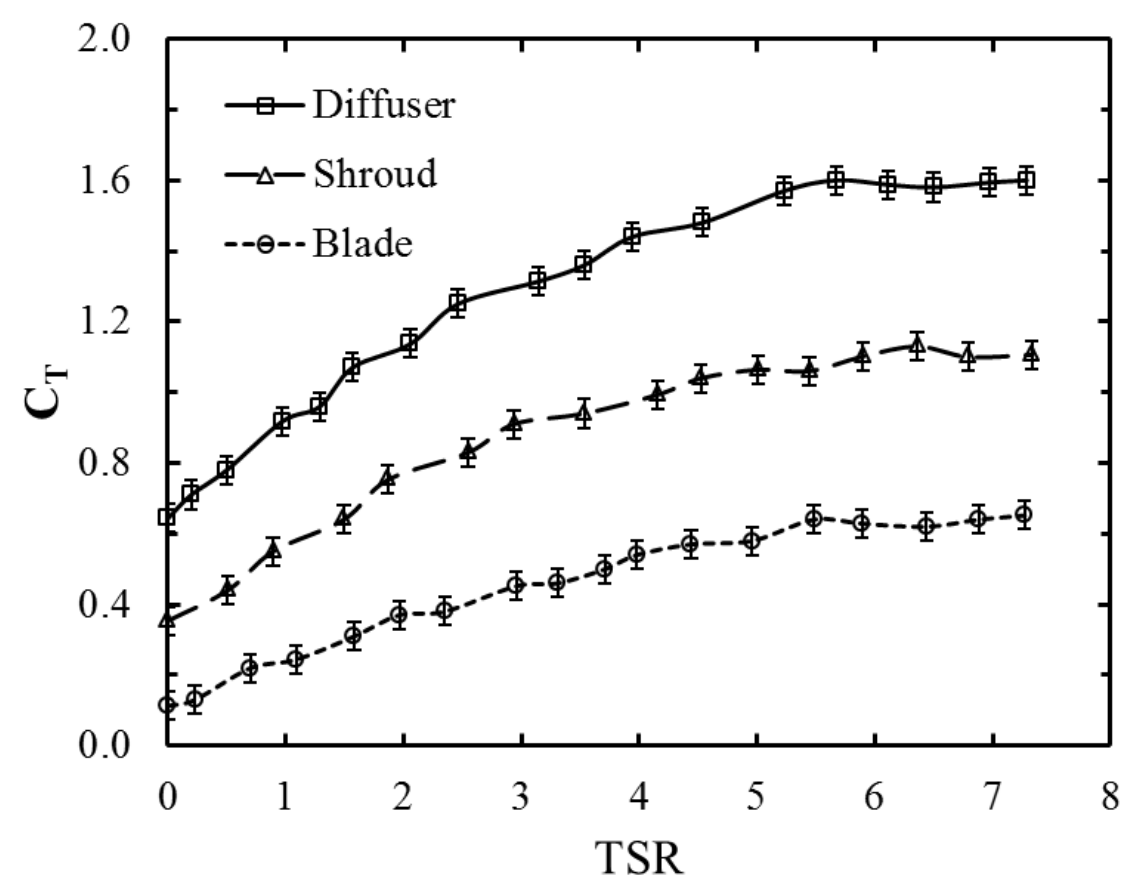

Figure 5.10: Thrust coefficient curves of the model turbine at $1.1 \mathrm{~m} / \mathrm{s}$ water velocity. 
As explained in Section 5.3, three distinct regions are identified in the performance curve of a turbine. The low tip speed ratio portion of the curve where the blades are stalled is characterized by low thrust and power coefficients. Wake expansion occurs slowly in this region of the turbine operation (Krogstad and Adaramola, 2012). The turbine at the optimum tip speed ratio region has moderate thrust coefficients and high power coefficients. The wake expands more in this region due to the higher energy extraction from the flow. Finally, the high tip speed ratio portion of the performance curve is identified by high thrust and low power coefficients. Wake expansion is the highest in this region due to the high level of drag forces (Adaramola and Krogstad, 2011).

\subsection{Relative Increase in Power Coefficients}

Figure 5.11 shows the relative increase in the maximum power coefficients of the shrouded model turbines compared to the unshrouded turbine. The relative increase in the maximum power coefficient of the model turbine with the shroud is $52 \%, 42 \%$, and $38 \%$ compared to the unshrouded turbine at the respective water velocities of 0.7, 0.9, and $1.1 \mathrm{~m} / \mathrm{s}$. The model turbine with the diffuser experiences $91 \%, 87 \%$, and $75 \%$ relative increase in the maximum power coefficient compared to the unshrouded turbine at the respective water velocities of the experiments. The maximum power enhancement of the shroud and the diffuser occurs at $0.7 \mathrm{~m} / \mathrm{s}$ water velocity and the enhancement decreases at higher flow velocities. The power enhancement of the shroud and the diffuser at $0.7 \mathrm{~m} / \mathrm{s}$ is respectively $14 \%$ and $16 \%$ more than the enhancement at $1.1 \mathrm{~m} / \mathrm{s}$ water velocity. This indicates a shrouded turbine is more effective in a low speed current and that the power enhancement of a shroud decreases as the stream velocity increases. 


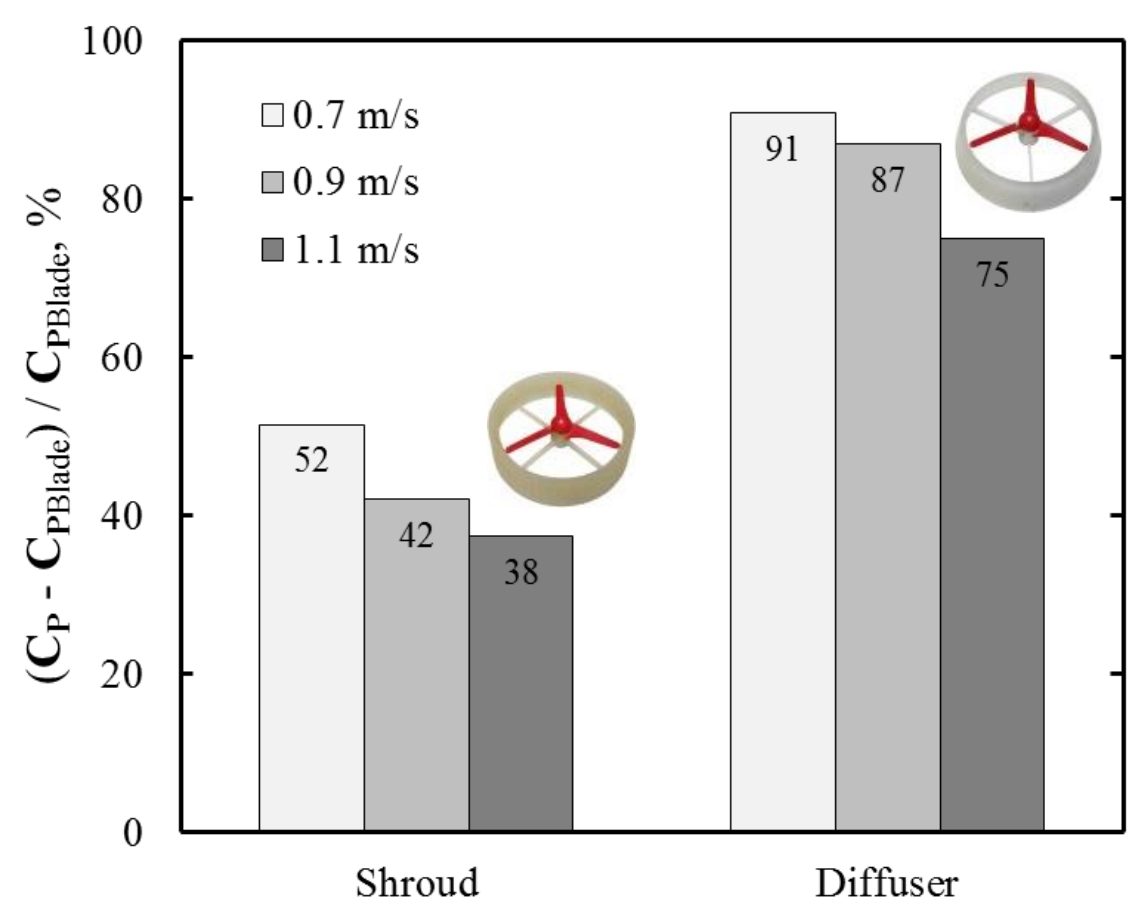

Figure 5.11: Relative increase in the maximum power coefficients of the shrouded model turbines compared to the unshrouded turbine.

\subsection{Reynolds Number Effect}

Figure 5.12 shows the maximum power coefficients of the shrouded and unshrouded model turbine versus the chord and diameter Reynolds numbers. The chord Reynolds number uses the blade chord length at $60 \%$ spanwise location and the blade tip velocity. The diameter Reynolds number uses the rotor blade diameter and the water velocity. The maximum power coefficients show a consistent increasing trend with the Reynolds number for the shrouded and unshrouded model turbine. The rate of increase decreases with the Reynolds number. The Reynolds dependent behaviour of the power coefficients decreases at higher flow velocities and eventually disappears (Krogstad and Adaramola, 2012). This indicates the highest achievable $C_{P}$ of the turbine with the diffuser is 0.7 and approximately no enhancement occurs at higher Reynolds numbers. 


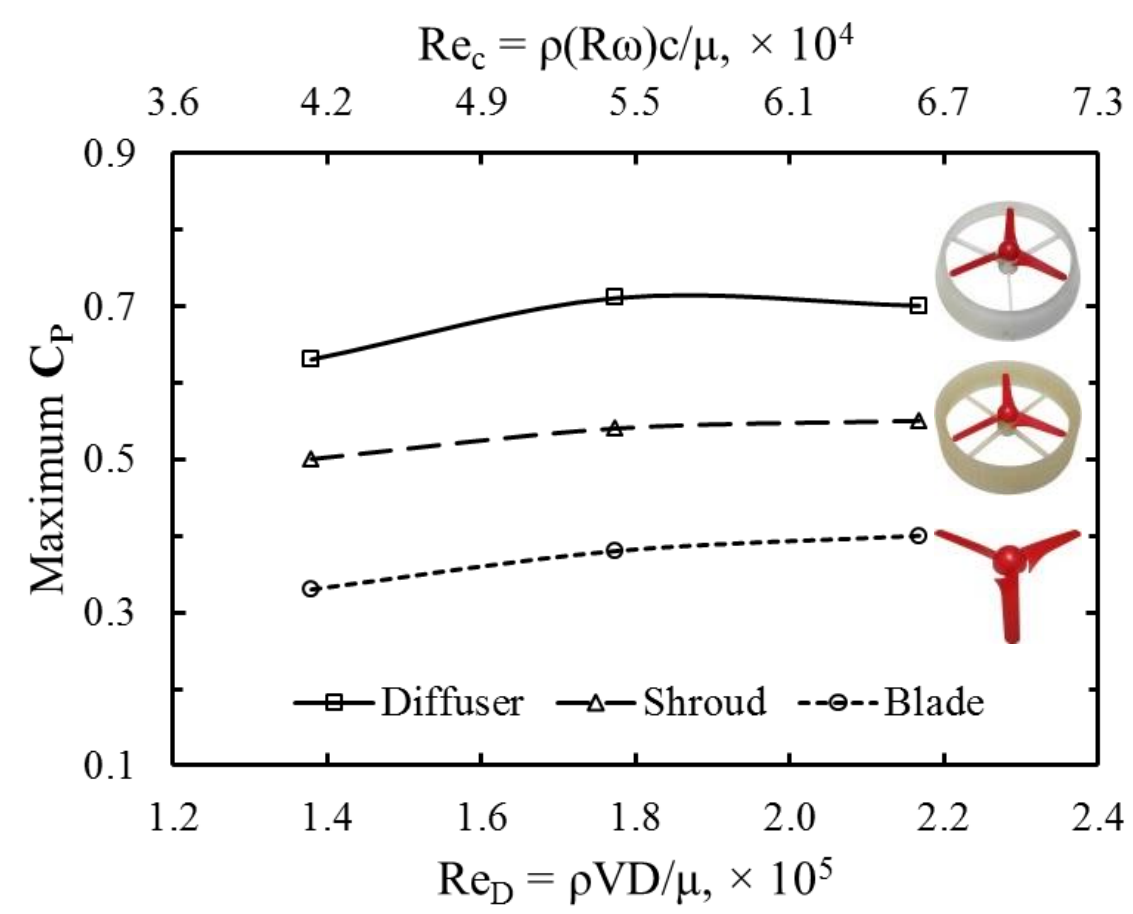

Figure 5.12: The maximum power coefficients of the shrouded and unshrouded model turbines increase with the Reynolds number at a decreasing rate.

For the experiments on a model turbine to realistically simulate the performance of a full scale turbine two conditions need to be met. Ideally, the Reynolds number and the tip speed ratio must remain the same for the field and water tunnel experiments. The condition on the tip speed ratio is the one that is met in the most wind and water tunnel experiments (Adaramola and Krogstad, 2011). Meeting this condition, it is then impossible to preserve the Reynolds number of a full scale turbine test. While the tip speed ratio is conserved, the Reynolds number could be one or two orders of magnitude larger for a full scale turbine (Wilson, 2009). A recommended range of the Reynolds number required for a reliable comparison of a model turbine and a full scale turbine is $10^{5}$ (de Vries, 1983). This criterion has been met in all of our experiments and therefore 
it is assumed that the results of this study remain valid for the higher Reynolds numbers of the full scale experiments.

\subsection{Extended Blade}

The performance coefficients in the foregoing sections were calculated based on the swept area of the blade resulting in the turbine with the diffuser to exceed the Betz limit. This is indeed comparing the output power of the shrouded turbine to the kinetic power of the stream available for the rotor swept area. It is beneficial and of course more realistic from an industrial point of view to compare the performance of a shrouded turbine to the available kinetic power for the largest area of the shroud, the exit area. In this manner the comparison is made between the performances of a shrouded turbine and an unshrouded turbine with a rotor that has a diameter as large as the shroud exit diameter. Discussion in this section focuses on the turbine with the diffuser, as the diffuser demonstrates the most performance enhancement.

Figure 5.13 shows the maximum power coefficients of the unshrouded turbine and also the turbine with the diffuser. Performance coefficients for the model turbine with the diffuser are also calculated based on the diffuser exit area. The top and the middle lines in the diagram represent the maximum power coefficients of the turbine with the diffuser calculated with the rotor swept area and the diffuser exit area, respectively. The bottom line is the maximum power coefficients of the unshrouded turbine. It is only the top line that exceeds the 0.59 Betz limit and the power coefficients calculated with the diffuser

exit area are as expected below the theoretical limit. Comparing the middle and the 
bottom lines indicates the maximum power coefficient of the turbine with the diffuser, based on the diffuser exit area, exceeds the unshrouded turbine performance by $9 \%$ and $8 \%$ at 0.7 and $0.9 \mathrm{~m} / \mathrm{s}$ water velocities, respectively. It is observed from the results at $1.1 \mathrm{~m} / \mathrm{s}$ water velocity that an unshrouded turbine with the same power characteristics of the blade and of the size equal to the diffuser exit diameter generates within $1 \%$ as much power as the model turbine with the diffuser.

We next calculate the diameter of an extended rotor blade capable of generating the same power as the model turbine with the diffuser. Assumption is that the extended rotor blade is a geometrically scaled up of the blade with the same power coefficient. Using the power coefficients of the rotor blade and the maximum power of the turbine with the diffuser allows calculation of the extended blade diameter. Figure 5.14 shows the ratio of the extended blade diameter to the diameter of the rotor blade as well as to the diffuser exit diameter. The top line is the diameter ratio of the extended blade and the rotor blade with values of $1.38,1.37$, and 1.32 at $0.7,0.9$, and $1.1 \mathrm{~m} / \mathrm{s}$ water velocities, respectively. This indicates that an extended blade requires a 38\%, 37\%, and 32\% larger diameter than the blade in the respective velocities to generate the same amount of power as the model turbine with the diffuser does. The bottom line in the diagram is the ratio of the extended blade diameter to the diffuser exit diameter with the values of $1.05,1.04$, and 1.01 . Similarly, an extended blade with a 5\%,4\%, and $1 \%$ larger diameter than the diffuser exit diameter generates the same power as the model turbine with the diffuser in the respective water velocities. On average, this is a rotor blade with only a $3 \%$ larger diameter than the diffuser exit diameter. This is in agreement with van Bussel (1999, 2007) who explained this result using his momentum theory (van Bussel, 1998). 
Assuming no viscous wake mixing behind the diffuser, van Bussel stated the power of a shrouded wind turbine increases as a result of the increased mass flow through the rotor blade. He further explained the amount of energy extracted per unit volume with a shrouded and an unshrouded wind turbine is the same. He then concluded the achievable performance of a diffuser augmented wind turbine is comparable to the performance of an unshrouded turbine having a diameter equal to the diffuser exit diameter. This has been also observed by Scherillo et al. (2011) in their experimental study on a streamlined diffuser with a $26^{\circ}$ half angle. They concluded the contribution of a diffuser to the power coefficient of a turbine is relatively small, $7.5 \%$ in their experiment, when the power coefficient is calculated based on the diffuser exit area.

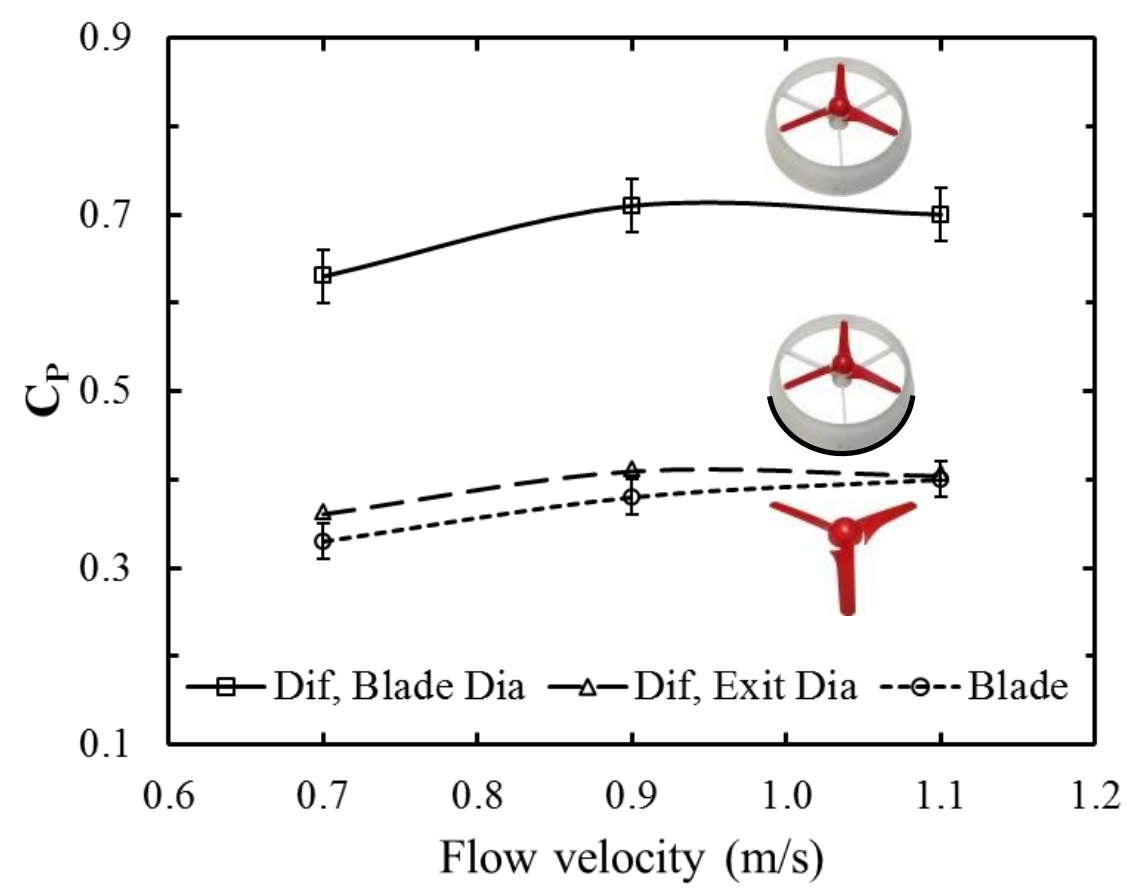

Figure 5.13: The maximum power coefficients of the model turbine with and without the diffuser calculated based on the blade swept area and the diffuser exit area. 


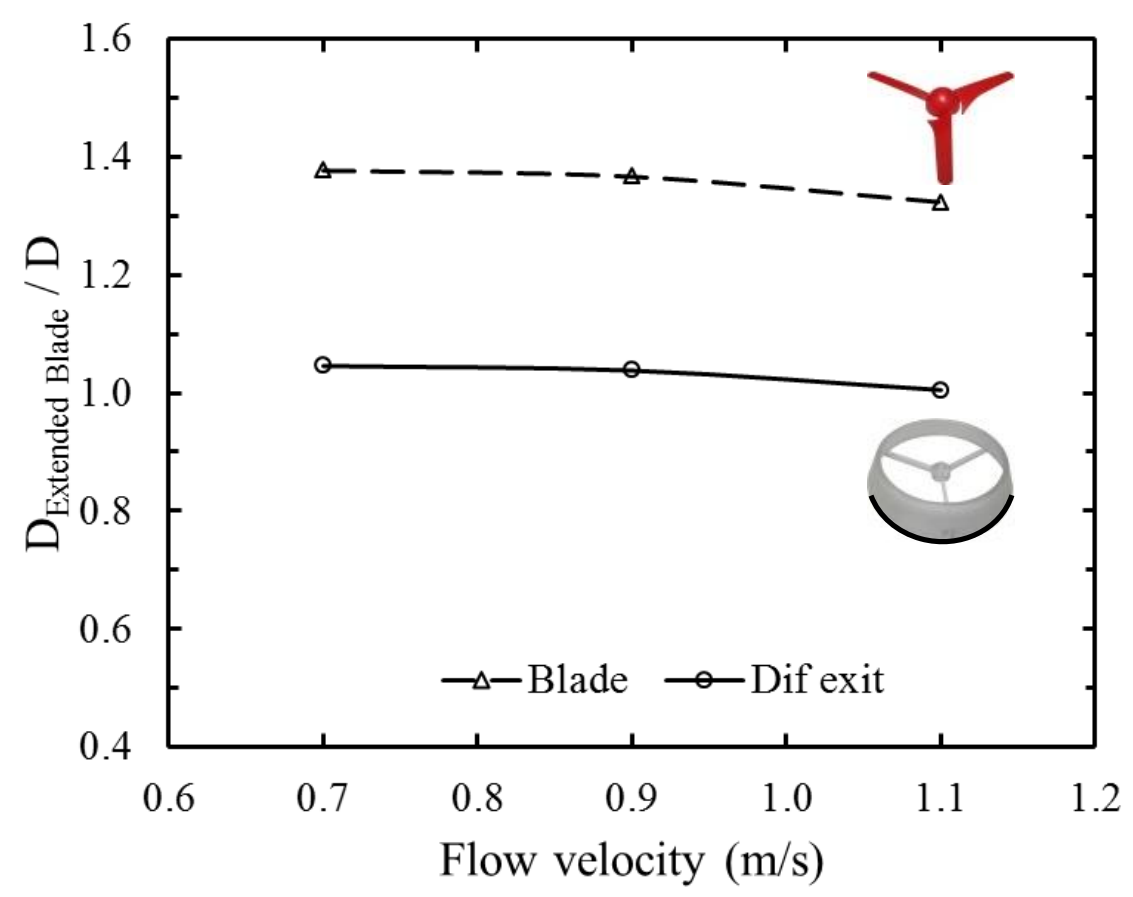

Figure 5.14: Diameter of an extended blade for generating the same power of the model turbine with the diffuser.

\subsection{Shroud or Extended Blade}

Figure 5.15 compares the volumes of the extended rotor blade and the blade with the diffuser. This provides an estimation of the size for the model turbine consists of the blade and the diffuser as well as for a turbine with the extended blade. It is seen that the extended blade has on average 2.5 times larger volume than the basic rotor blade in the three water velocities. In contrast, the volume of the rotor blade and the diffuser is 28 times greater than the volume of the basic rotor blade. This means the model turbine with the diffuser has 11 times larger volume than the model turbine with the extended blade. 


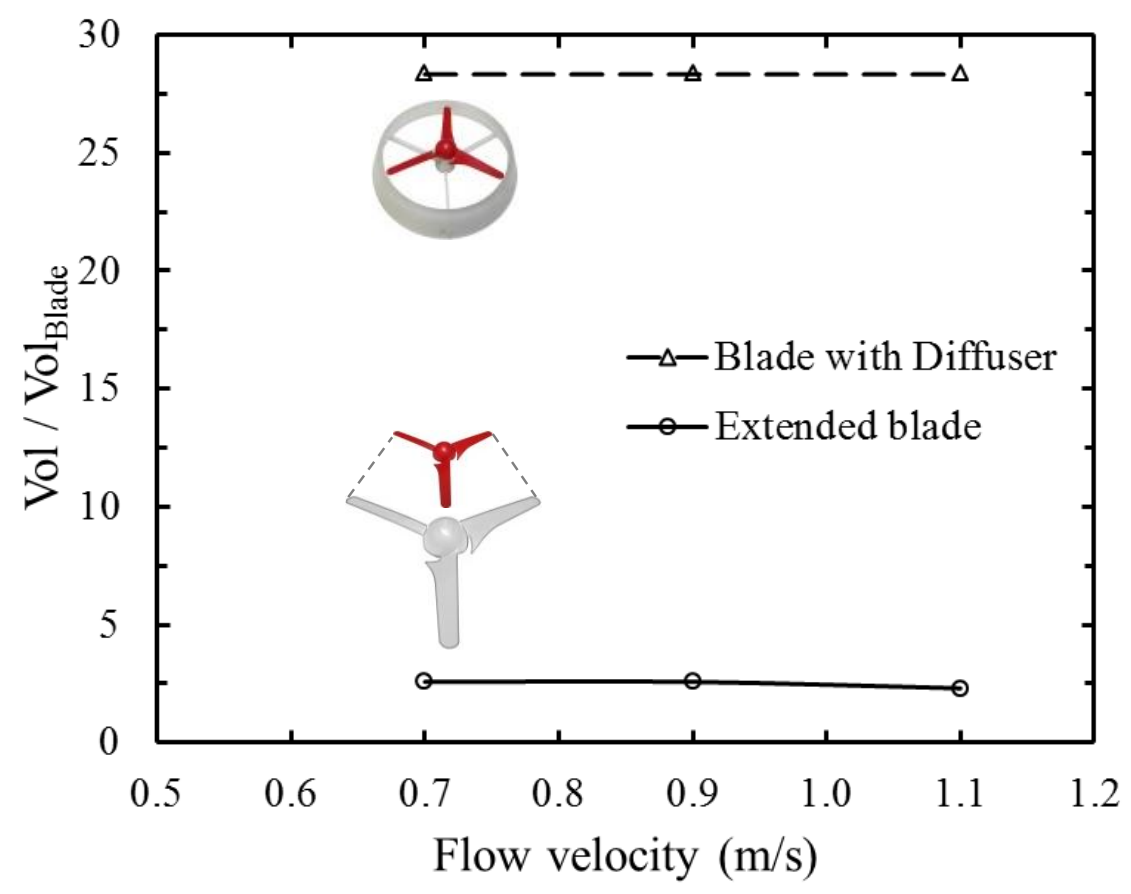

Figure 5.15: Comparison of the volumes for the turbine with the diffuser and the turbine with an extended blade generating the same power.

This is an important result when it comes to the industrial manufacturing of hydrokinetic turbines where the less material normally leads to the lower product cost. Since an extended blade can substitute for a blade and a diffuser, employing a shrouded turbine may not be justified for just increasing the performance of a turbine. A shroud needs to benefit the hydrokinetic turbine system in other ways. This statement is in contrast to many literature studies that highlight the apparent increase in the power coefficient as the main motivation factor for using a shroud. Benefits of a shroud include reducing the destructive effect on the performance when the blades pass too close to the flow boundaries (Fraenkel, 2002). This happens in shallow water streams or when the turbines need to be deployed close to the water surface or on the riverbed. A shroud enclosing a turbine rotor prevents the counterproductive effect of the surface on the turbine performance. A shroud can secure the turbine generator and powertrain and also can be 
used as the structure to install the turbine on the riverbed, or as the buoy to float it in a water stream (Khan et al., 2009). Significant advantage in the powertrain design can be obtained with a shroud when striving, for instance, to avoid use of a gearbox to reduce the maintenance and eliminate oil spillage to facilitate turbine permitting process. Additionally, a shroud can benefit the marine life providing protection for fish or diving birds from entering the rotor from sides (Fraenkel, 2006). A turbine with a smaller rotor blade encased in a shroud may have less impact on the marine species compared to a larger unprotected rotating blade (Wilson et al., 2007). In off-axis flow conditions a shroud results in a less performance deterioration by streamlining the flow through the rotor blade (see Chapter 6). A shroud however, can make hydrokinetic turbine deployment and retrieval more difficult and impact on the water safety for operators. A shroud can also produce a larger wake region impacting the aquatic life, especially when the flow is not aligned with the turbine axis. This possibly affects how the turbine farms placement is approved for permitting. Designing a shroud which overall attributes could compete in the marketplace needs a complete water-to-wire analysis.

Selecting either approaches, a smaller blade with a shroud or a larger blade, requires optimization on multiple variables which is not well documented. Fabrication, deployment and retrieval, maintenance, overall cost, water depth, flow velocity, and marine life should be part of such an optimization study. Although utilizing a larger rotor blade without a shroud might seem a lower cost approach, a smaller blade with a shroud could be a better option for the sites with low water velocities, shallow water, a maintenance free powertrain design, and where the marine life safety is of concern. 


\section{Chapter 6 Performance in Yaw Operation}

\subsection{Foreword}

A wind turbine rotor in turbulent atmospheric boundary layer is nearly always in yawed flow conditions (Smulders et al., 1981). Rapid variations occur in the wind direction and a wind turbine lags behind the rapid changes. In practice, yaw operations are more usual than inline operations for a wind turbine (van Bussel, 1994). It is common that a wind turbine rotor operates at yaw angles as large as $30^{\circ}$ for several minutes (Hansen and Butterfield, 1993). Similarly, a hydrokinetic turbine encounters changes in the direction of water streams that affects the performance of turbines. From testing hydrokinetic turbines on the Winnipeg River, flow variations have been noticed attributed to existence of ice (Birjandi et al., 2015). A turbine that encounters relatively high rates of yaw misalignment experiences fatigue loads induced by gyroscopic moments that could result in failure. Lynette (1988) describes turbine damages caused by yaw misalignment as the second cause of a wind turbine downtime. Yaw operation of a wind turbine results in a skewed wake, modified induced velocities, and cyclic changes in the blades' angles of attack. This leads to dynamic stall and variable loading on the rotor blade and therefore loss of yaw stability which consequently creates excessive induced loads on the rotor and turbine structure (Hansen, 1992). Evaluating hydrokinetic turbines in yawed flow 
conditions helps predicting the turbine performance and power stability in off-axis flows. The effect of yaw angle also plays an important role in designing yaw control mechanisms to optimize the performance of turbines (Boorsma, 2012). This role is more pronounced for a floating turbine where a yaw control mechanism is more complicated than for a fixed turbine (Krogstad and Adaramola, 2012). Understanding the effect of yaw angle on the performance of shrouded hydrokinetic turbines is necessary in designing a robust turbine for harsh river and ocean environments. Nevertheless, a comprehensive and conclusive study on the effect of yaw angle on the performance of shrouded hydrokinetic turbines is not available in the literature (see Chapter 2). The works of Igra and Grumman Corp. which are the two comprehensive studies on diffuser augmented wind turbines (Section 2.2) are not conclusive on the effect of yaw angle. Not only yaw angle influences the performance of a single turbine, but also plays an important role in the performance of a turbine farm. Deflection of the wake of the first row turbines in a farm could benefit operation of the downstream turbines and increase the farm overall output power (Adaramola and Krogstad, 2011; McKay et al., 2013).

Periodic variations in the blades' angles of attack due to yaw misalignments invalidate the assumptions, like axisymmetric inflow, used in the linear momentum theory, BEM based models, and wake models. Moreover, the induced rise in the radial velocity component in the boundary layer creates a more complex three dimensional unsteady flow over a rotor blade and in the wake flow (ElQatary and Elhadidi, 2014). Despite the simplicity of theoretical and BEM based methods, the effects of spanwise flow and skewed wake are neglected in such methods and thus inaccuracy is introduced in the yaw performance calculations. A Number of authors have pointed out such shortcomings and 
expressed the necessity and importance of experimental data in yaw operations (de Vries, 1985; Hansen and Cui, 1989; Hansen et al., 1990; Swift, 1981). The effect of such complicated flows can be implemented in an approximate way in the BEM methods using empirical values from experimental results (Gaonkar and Peters, 1986; Hansen, 1992; Hasegawa et al., 1996; van Bussel, 1994). A better understanding of such flows however needs more representative models based on the experimental studies (Vermeer et al., 2003). To account for the effect of yaw angle in semi-empirical models like the prescribed wake description model (Robinson et al., 1995) and to use such a tool with confidence in designing horizontal axis turbines, experimental results need to be implemented in the models (Grant et al., 1997).

Performance characteristics of the shrouded and unshrouded model turbines in yaw operations are presented in this chapter. The results for the unshrouded turbine are also compared to the results of the linear momentum theory. In an analogy to that, the performance of a shrouded turbine is reported for the first time to decrease according to a power of cosine of yaw angle. This provides a valuable insight in studying the performance of shrouded hydrokinetic turbines in commercial applications.

\subsection{Results and Discussions}

The experimental power, torque, and thrust coefficients for the shrouded and unshrouded model turbines in yaw operations are presented in this section. The coefficients are calculated based on the blade swept area and are corrected for the free surface proximity and blockage effects. The results are for the unshrouded turbine, turbine with the shroud, 
and turbine with the diffuser. The three configurations are respectively referred to as the blade, shroud, and diffuser in the diagrams. The results are presented for $0.7,0.9$, and $1.1 \mathrm{~m} / \mathrm{s}$ water velocities and for $5^{\circ}$ to $25^{\circ}$ yaw angles. The experiments are done for the two possible directions of yaw rotation in the horizontal plane. The performance curves of the shrouded and unshrouded model turbines show no sensitivity to the sign of yaw angle. The experimental data for the shrouded and unshrouded model turbines in yaw operations is available in (Shahsavarifard and Bibeau, 2014b).

\subsection{Power Coefficients}

Figure 6.1 shows the power coefficients of the shrouded and unshrouded model turbines for yaw angles of $5^{\circ}$ to $25^{\circ}$. The performance coefficients are for $0.7 \mathrm{~m} / \mathrm{s}$ water velocity obtained in $5^{\circ}$ increments. The maximum power coefficients for the three configurations of the model turbine are summarized in Table 6.1. It is apparent from the experimental results that the turbine performance decreases as yaw angle increases. Up to $10^{\circ}$ yaw angle the reduction of the power coefficients with respect to the inline operation for the three turbine configurations is negligible. The amount of power reduction gradually increases up to $25^{\circ}$ yaw angle where the maximum reduction occurs. The maximum performance reduction with respect to the inline operation is $24 \%$ for the blade. The maximum reduction in the performance of the model turbine with the shroud and with the diffuser is respectively $8 \%$ and $22 \%$ compared to the inline performances. The experimental results show the shroud effectively reduces the influence of yaw angle on the turbine performance at $0.7 \mathrm{~m} / \mathrm{s}$ water velocity. The diffuser displays virtually no influence on reducing the effect of yaw angle on the turbine performance. 


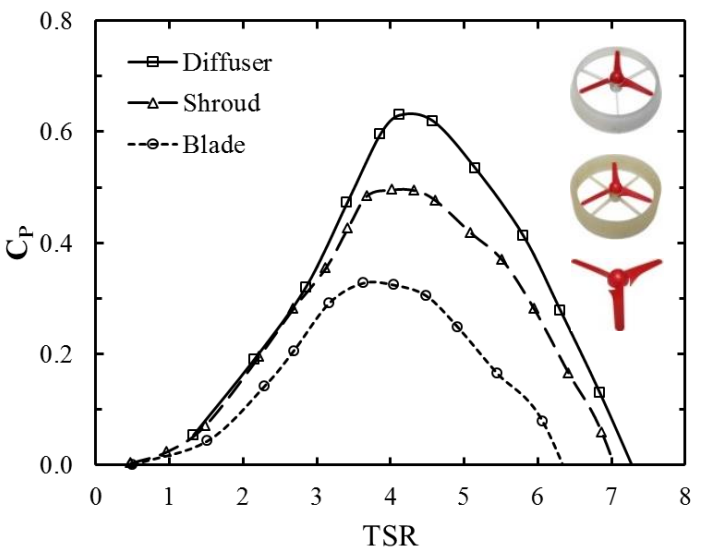

(a) $\gamma=5^{\circ}$

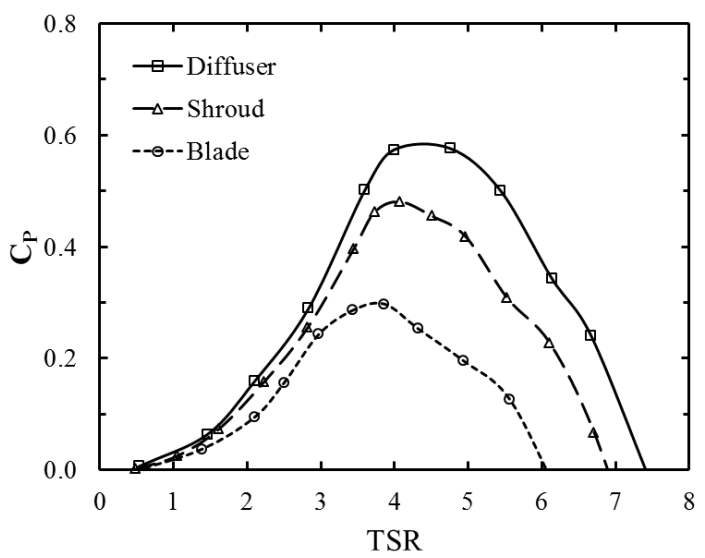

(c) $\gamma=15^{\circ}$

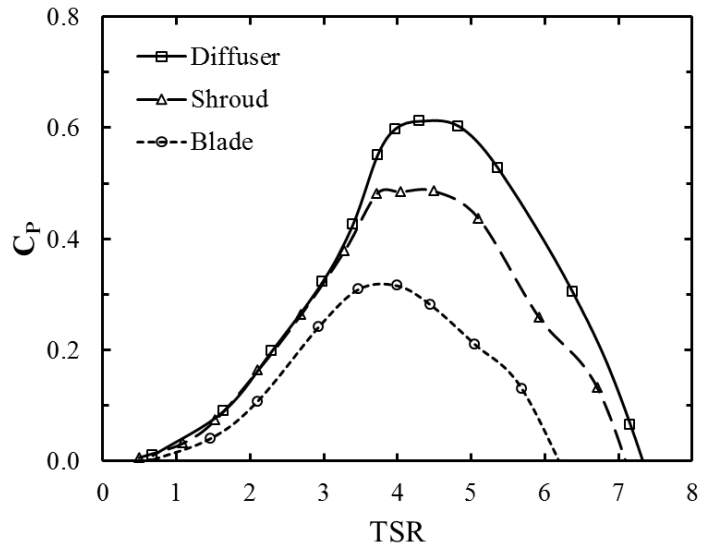

(b) $\gamma=10^{\circ}$

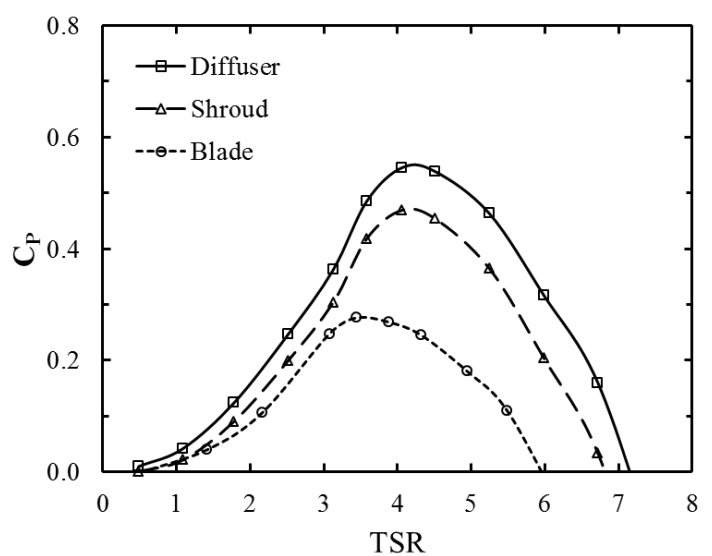

(d) $\gamma=20^{\circ}$

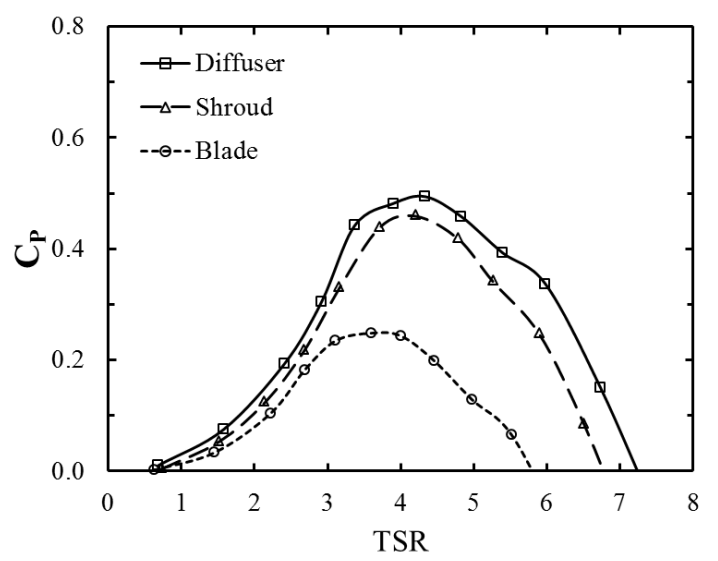

(e) $\gamma=25^{\circ}$

Figure 6.1: Power coefficients of the model turbine in yaw operations at $0.7 \mathrm{~m} / \mathrm{s}$ water velocity. 
Table 6.1: Maximum power coefficients for the model turbine at $0.7 \mathrm{~m} / \mathrm{s}$ water velocity

\begin{tabular}{cccccc}
\hline & \multicolumn{5}{c}{ Yaw angle } \\
\cline { 2 - 6 } & $5^{\circ}$ & $10^{\circ}$ & $15^{\circ}$ & $20^{\circ}$ & $25^{\circ}$ \\
\hline Blade & 0.33 & 0.32 & 0.30 & 0.28 & 0.25 \\
Shroud & 0.50 & 0.49 & 0.48 & 0.47 & 0.46 \\
Diffuser & 0.63 & 0.61 & 0.58 & 0.55 & 0.49 \\
\hline
\end{tabular}

Figure 6.2 shows the power coefficients of the shrouded and unshrouded model turbines for the range of yaw angles investigated. The power coefficients are for $0.9 \mathrm{~m} / \mathrm{s}$ water velocity obtained in the increments of $5^{\circ}$ from $5^{\circ}$ to $25^{\circ}$ yaw angles. The maximum power coefficients for the three configurations of the model turbine are summarized in Table 6.2. Similarly to the tests in $0.7 \mathrm{~m} / \mathrm{s}$ water velocity, it is apparent from the experimental results that the turbine performance decreases as yaw angle increases. The reduction in the performance of the model turbine with respect to the inline operation is insignificant for yaw angles up to $10^{\circ}$ but increases to its maximum value at $25^{\circ}$. At $25^{\circ}$ yaw angle $24 \%$ reduction is observed in the performance coefficient of the unshrouded turbine compared to zero yaw operation. The maximum reduction in the performance of the model turbine with the shroud is $6 \%$ and for the turbine with the diffuser is $21 \%$ compared to the respective values in inline operation. As in the case of $0.7 \mathrm{~m} / \mathrm{s}$ water velocity, the shroud is observed to significantly decrease the effect of yaw angle on the turbine performance. The diffuser again shows minimal ability to lessen the performance reduction in yaw operations as percentage of the power reduction for the turbine with the diffuser is close to that of the unshrouded turbine. 


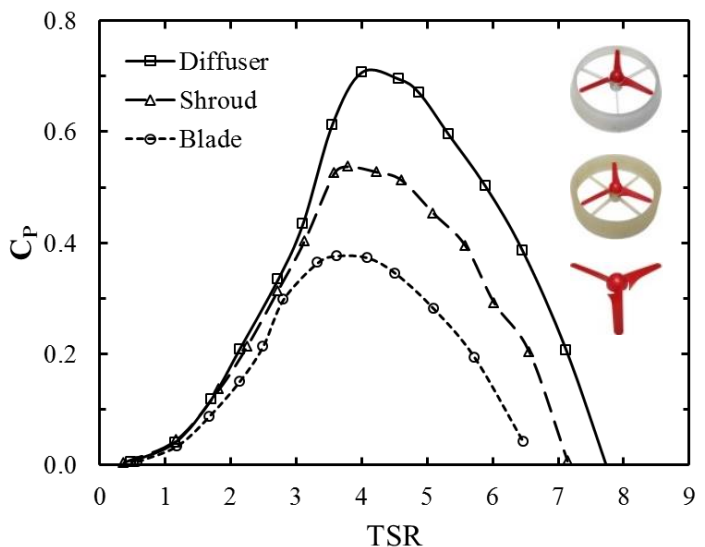

(a) $\gamma=5^{\circ}$

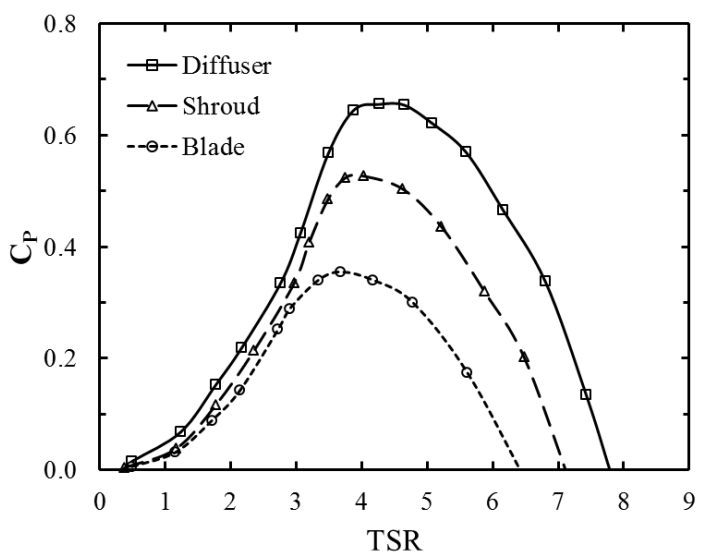

(c) $\gamma=15^{\circ}$

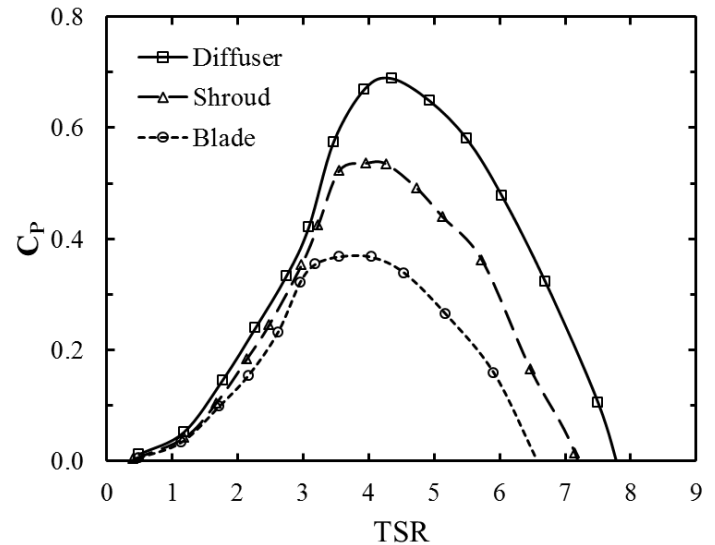

(b) $\gamma=10^{\circ}$

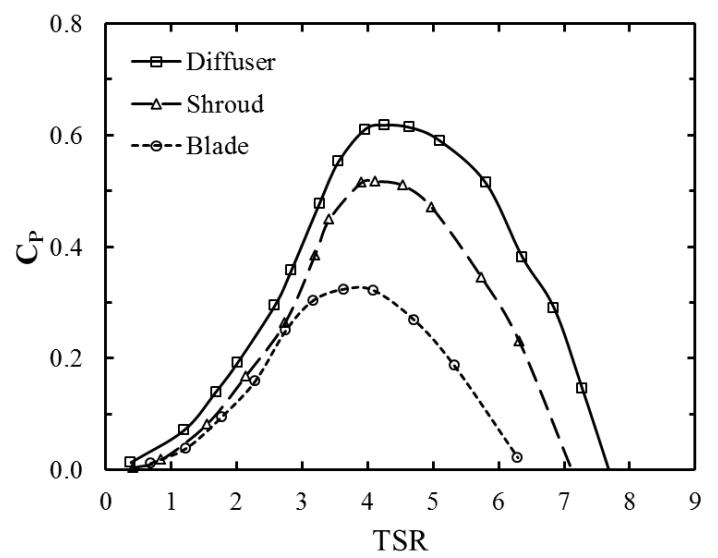

(d) $\gamma=20^{\circ}$

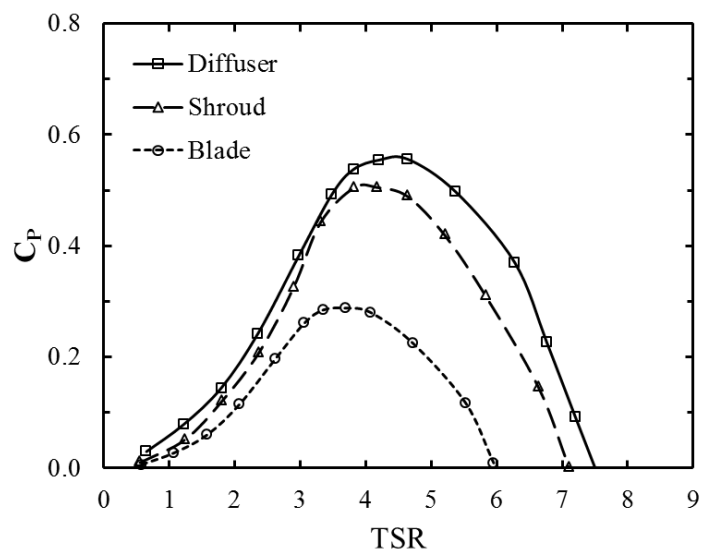

(e) $\gamma=25^{\circ}$

Figure 6.2: Power coefficients of the model turbine in yaw operations at $0.9 \mathrm{~m} / \mathrm{s}$ water velocity. 
Table 6.2: Maximum power coefficients for the model turbine at $0.9 \mathrm{~m} / \mathrm{s}$ water velocity

\begin{tabular}{cccccc}
\hline & \multicolumn{5}{c}{ Yaw angle } \\
\cline { 2 - 6 } & $5^{\circ}$ & $10^{\circ}$ & $15^{\circ}$ & $20^{\circ}$ & $25^{\circ}$ \\
\hline Blade & 0.38 & 0.37 & 0.35 & 0.32 & 0.29 \\
Shroud & 0.54 & 0.54 & 0.53 & 0.52 & 0.51 \\
Diffuser & 0.71 & 0.69 & 0.66 & 0.62 & 0.56 \\
\hline
\end{tabular}

Figure 6.3 shows the power coefficients of the shrouded and unshrouded model turbines for yaw angles of $5^{\circ}$ to $25^{\circ}$. The performance coefficients are for $1.1 \mathrm{~m} / \mathrm{s}$ water velocity obtained in $5^{\circ}$ increments. The maximum power coefficients for the three configurations of the model turbine are summarized in Table 6.3 . As in the cases of $0.7 \mathrm{~m} / \mathrm{s}$ and $0.9 \mathrm{~m} / \mathrm{s}$ tests, it is apparent from the experimental results that the turbine performance decreases as yaw angle increases. Again, for yaw angles up to $10^{\circ}$, the reduction in the turbine performance with respect to the inline operation is negligible for the three turbine configurations. The amount of performance reduction gradually increases to its maximum value at $25^{\circ}$ yaw angle. The maximum performance reduction for the unshrouded turbine, the blade, compared to zero yaw operation is $22 \%$. The maximum reduction in performance of the model turbine with the shroud and with the diffuser is respectively $5 \%$ and $21 \%$ compared to the inline performances. As in the $0.7 \mathrm{~m} / \mathrm{s}$ and $0.9 \mathrm{~m} / \mathrm{s}$ test cases, the shroud effectively reduces the effect of yaw angle on the turbine performance. The diffuser also shows no capability in reducing the effect of yaw angle on the performance of the model turbine. 


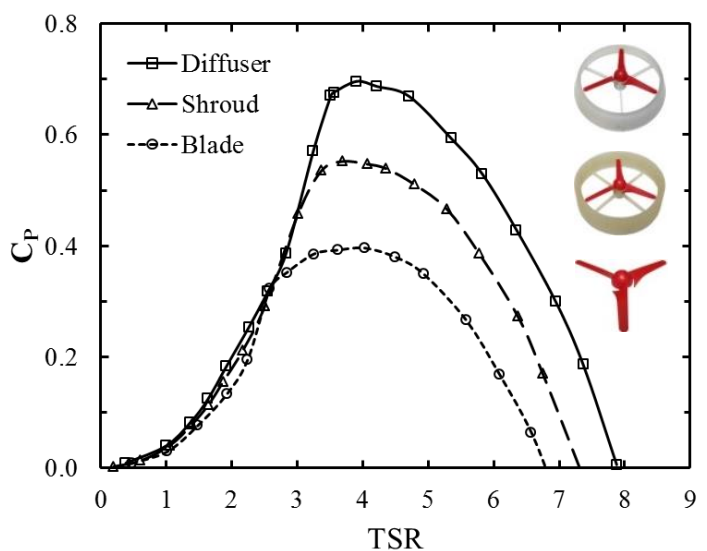

(a) $\gamma=5^{\circ}$

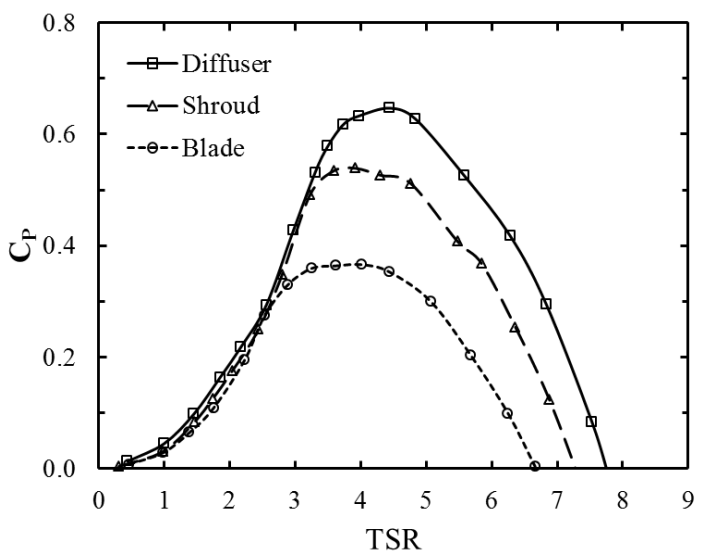

(c) $\gamma=15^{\circ}$

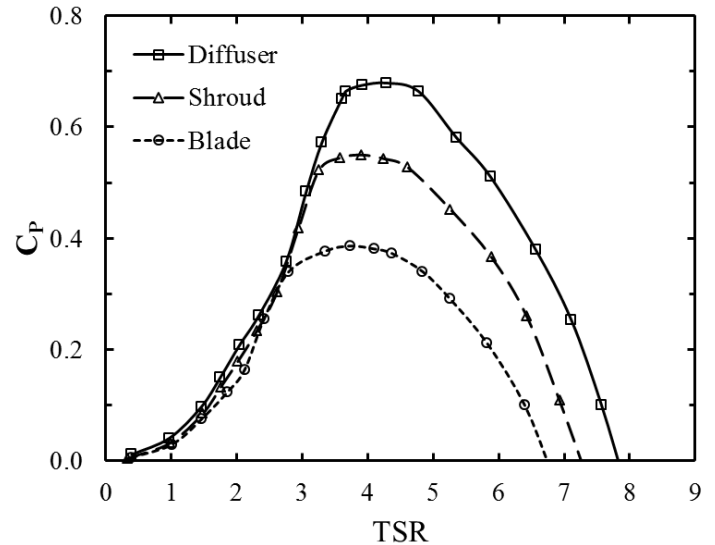

(b) $\gamma=10^{\circ}$

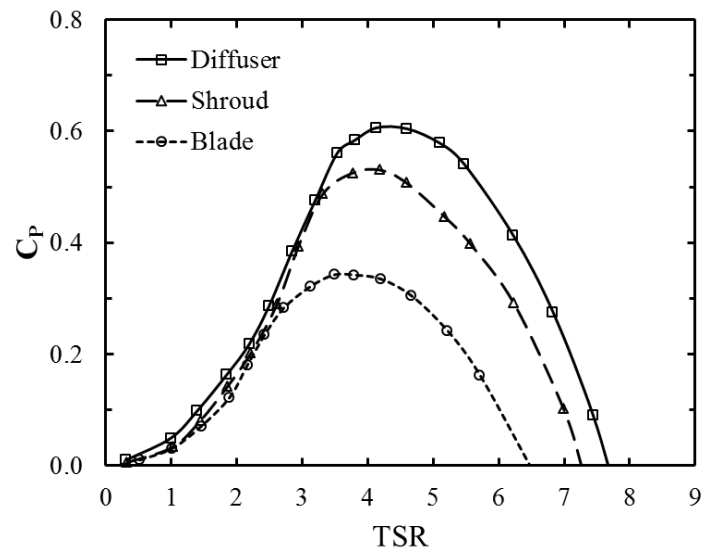

(d) $\gamma=20^{\circ}$

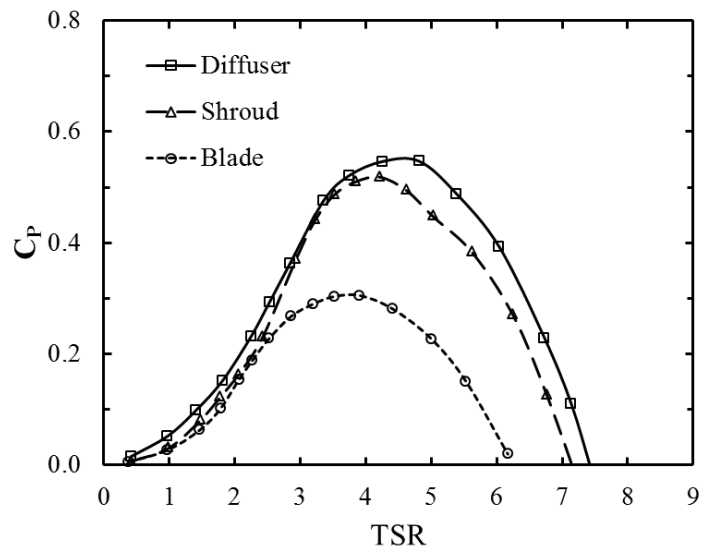

(e) $\gamma=25^{\circ}$

Figure 6.3: Power coefficients of the model turbine in yaw operations at $1.1 \mathrm{~m} / \mathrm{s}$ water velocity. 
Table 6.3: Maximum power coefficients for the model turbine at $1.1 \mathrm{~m} / \mathrm{s}$ water velocity

\begin{tabular}{cccccc}
\hline & \multicolumn{5}{c}{ Yaw angle } \\
\cline { 2 - 6 } & $5^{\circ}$ & $10^{\circ}$ & $15^{\circ}$ & $20^{\circ}$ & $25^{\circ}$ \\
\hline Blade & 0.40 & 0.39 & 0.37 & 0.34 & 0.31 \\
Shroud & 0.55 & 0.55 & 0.54 & 0.53 & 0.52 \\
Diffuser & 0.70 & 0.68 & 0.65 & 0.61 & 0.55 \\
\hline
\end{tabular}

The shape of the power curves for the model turbine in yaw angle is similar to the performance curves in inline operations. Performance coefficients of the shrouded and unshrouded model turbines start from zero increase with the tip speed ratio and achieve a maximum at a tip speed ratio of approximately 4 . Further increase in the tip speed ratio results in descending the power curve to zero at a tip speed ratio of approximately 7 . Performance of the unshrouded turbine, the blade, and the model turbine with the diffuser is observed to decrease with increasing the yaw angle. However, the performance curve of the model turbine with the shroud remains nearly unchanged with the changes in the yaw angle at the three water velocities examined. At $25^{\circ}$ yaw angle, the performance curve for the model turbine with the diffuser falls close to the power curve of the turbine with the shroud. At the optimum power, the difference between the two curves is less than $10 \%$ at the three water velocities. This shows the ability and weakness of the shroud and the diffuser in keeping the turbine power curve unchanged in yaw operations.

It is observed from the performance diagrams that the low tip speed ratio portion of the curves for the three turbine configurations overlap at each yaw angle. This means that the 
shroud and the diffuser are unable to enhance the turbine performance in the low tip speed ratio portion of the performance curve. Performance reduction in yaw operations has two main reasons: first, the changes occur in the aerodynamic behaviour of the blades due to variations of the angles of attack and second, the reduced projected swept area (Pedersen, 2004). At the low tip speed ratio portion, the blades are in a deep stall mode (Schreck et al., 2001); the stall and not the yaw effect is the dominant phenomenon and a shroud is unable to push the blades out of the stall (Phillips, 2003). This characteristic is similar to what is discussed in Section 5.3 for zero yaw experiments. Regardless of the operating yaw angle, blades at the low tip speed ratio are stalled due to the high angles of attack. In this situation a shroud is unable to make enough changes in the angles of attack required to alter the stall state. Increasing the tip speed ratio reduces the angles of attack and allows the blades to leave the stall. The stall is no longer dominant and the yaw angle effect becomes the determinant factor. From this point on we see the influence of yaw angle on reducing the power coefficients of the shrouded and unshrouded turbine. At the optimum tip speed ratio, the blades operate at the highest lift to drag ratio and the turbine generates its maximum power. At the high tip speed ratio portion of the curves, low angles of attack and the consequent high drag is responsible for the lower output power. This is where the shroud and the diffuser are able to increase the angles of attack, reduce the drag, and enhance the turbine performance in inline operations. In yaw operations however, changes in the aerodynamic characteristics of the blades and the reduction in the projected swept area due to the increase in the yaw angles reduce the lift force on the blades and hence the turbine power. The shroud, having a well-designed inlet, is able to moderate these effects and keep the power curve nearly unchanged. The diffuser, having 
no inlet, fails to keep the performance unchanged and therefore power curve of the model turbine with the diffuser descends close to the curve of the turbine with the shroud.

Figure 6.4 shows the relative decrease in the maximum performance of the shrouded and unshrouded model turbines at $25^{\circ}$ yaw angle compared to the inline performances at the three water velocities. The amount of decrease for the blade and the turbine with the diffuser are almost the same with a maximum 3\% difference. This means the diffuser which increases the inline performance of the model turbine the most is unable to keep the high performance in yaw operations. Instead, the model turbine with the shroud is seen to have the least performance reduction in yaw operations. The shroud effectively moderates the effect of yaw angle on the turbine performance. The maximum reduction in the performance of the model turbine with the shroud is $6 \%$ in average at the three Reynolds numbers. The model turbine with the shroud experiences negligible performance loss in yaw operations compared to the unshrouded turbine. The performance loss of the turbine with the shroud in yaw operations is averagely $17 \%$ less than the power reduction of the unshrouded turbine.

It has been experimentally shown so far, that a horizontal axis hydrokinetic turbine experiences performance drop in off-axis flows. Detailed examination of the performance curves reveals another fact about yaw operations of a horizontal axis turbine: the runaway point of the blade, the unshrouded turbine, occurs at a lower tip speed ratio than in the inline operation. Runaway is the high tip speed ratio end point of a performance curve where the turbine does not generate any power. In other words, not only the turbine performance decreases but also the operating tip speed ratio range of the turbine 
decreases in yaw operations. This behaviour has been experimentally observed and reported for an unshrouded turbine (Krogstad and Adaramola, 2012). To investigate this characteristic in detail, performance curves of the shrouded and unshrouded model turbines are shown in a different order in Figures 6.5 to 6.7. The performance curves of the three turbine configurations are shown separately for the range of tip speed ratio examined at the three Reynolds numbers of the experiments. Power curves of the blade in yaw operations are depicted in Figure 6.5 and those for the turbine with the shroud and with the diffuser are shown in Figures 6.6 and 6.7, respectively. A common aspect of the figures is that the low tip speed ratio portions of the curves show no sensitivity to the yaw angle, irrespective of the turbine configuration and the Reynolds number. This has been reported in the literature for an unshrouded turbine (Noura et al., 2012).

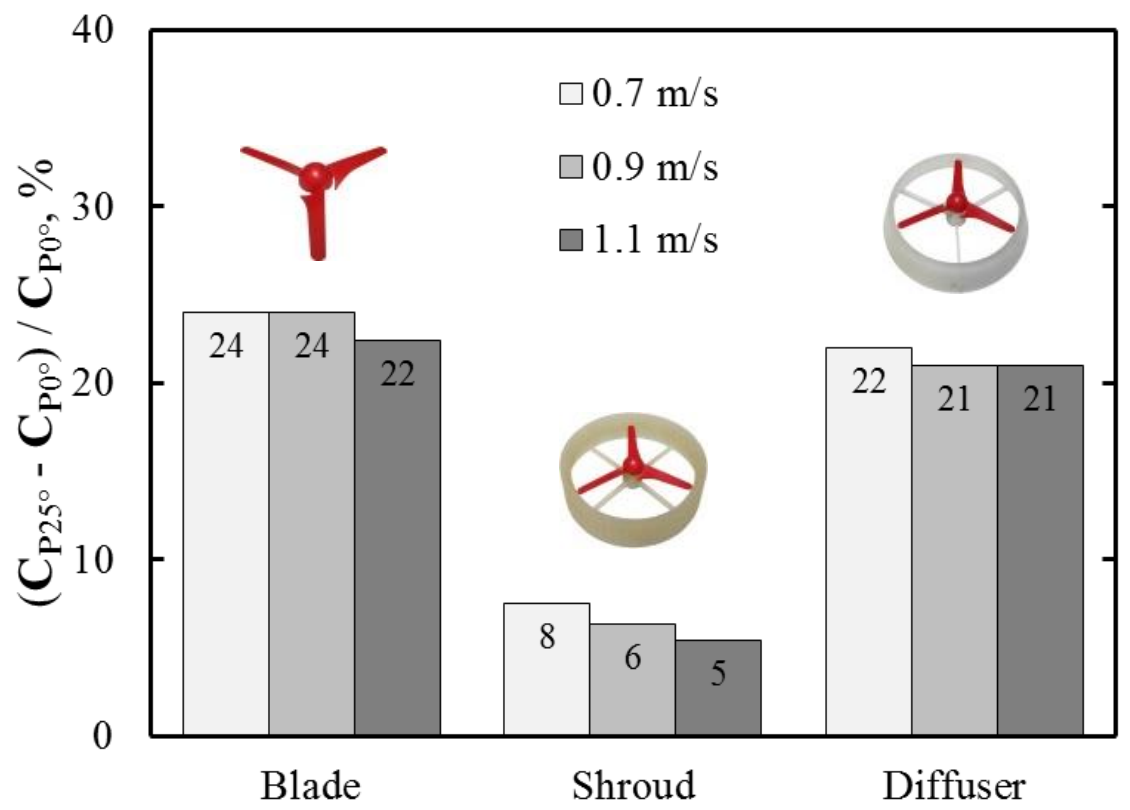

Figure 6.4: Relative decrease in the maximum performance coefficients of the shrouded and unshrouded model turbines at $25^{\circ}$ yaw angle compared to zero yaw angle. 
Figure 6.5 shows the performance curves of the blade for the range of yaw angles investigated at the three Reynolds numbers of the experiments. Three features are observed in the diagrams. First, the overlap of the low tip speed ratio portion of the curves that happens at the three Reynolds numbers explained previously. Second, the reduction in the maximum power coefficient of the turbine in yawed flows is apparent at the three water velocities. Changes in the angles of attack of the blades due to the yaw angle makes the rotor blade operate away from the optimum tip speed ratio and subsequently the optimum angle of attack (Maeda et al., 2008). The larger the yaw angle is the further the blades are from the optimum angle of attack and the less power the turbine generates. Third, as yaw angle increases the runaway point moves to the left to a lower tip speed ratio. This results in a narrower operating range of the turbine. Further reduction in the angles of attack causes increase in the drag force on the blades and results in a runaway point with a smaller tip speed ratio (Tsalicoglou et al., 2012). The reduction of the projected swept area in yaw operations is also a determinant factor in the optimum and high tip speed ratio portions of the curves (Pedersen, 2004). As the yaw angle increases, the projected area decreases and this added to the effect of the reduced angles of attack further decreases the output power. Operating a hydrokinetic turbine in yaw conditions not only reduces its performance but also narrows its operating range. This is intensified as the yaw angle increases. 


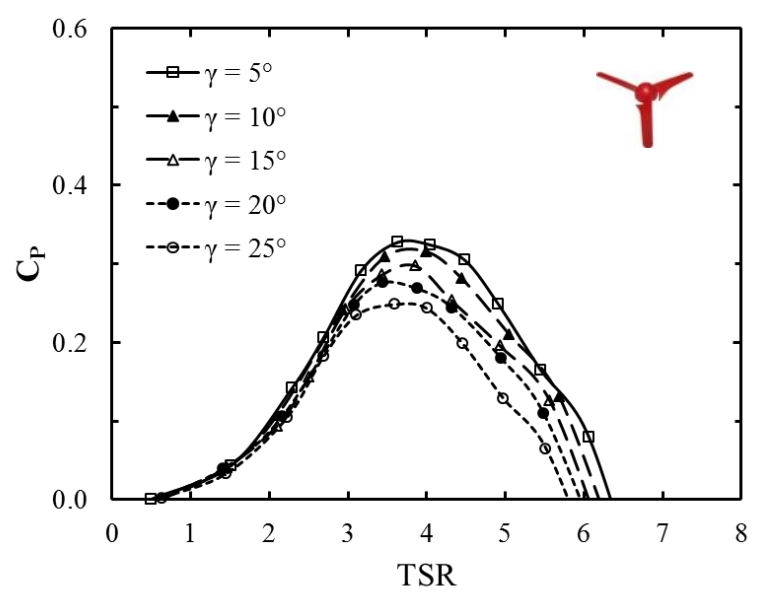

(a) $0.7 \mathrm{~m} / \mathrm{s}$ water velocity

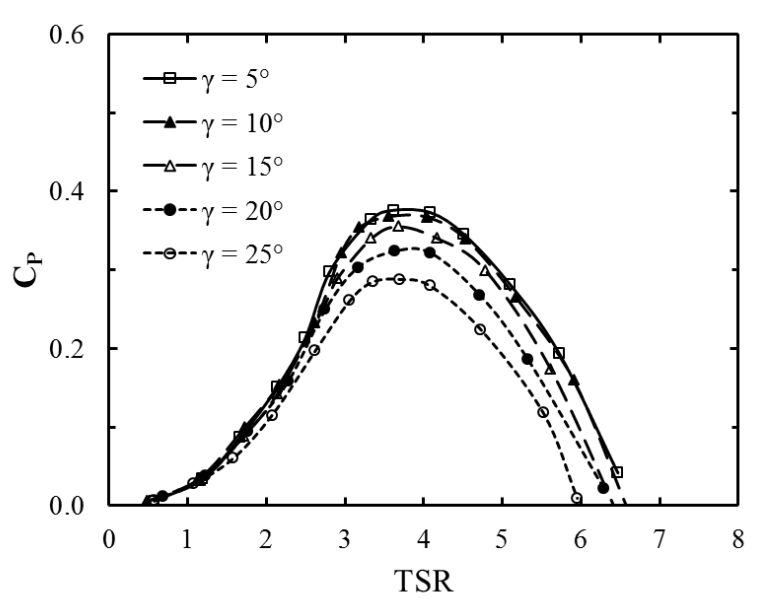

(b) $0.9 \mathrm{~m} / \mathrm{s}$ water velocity

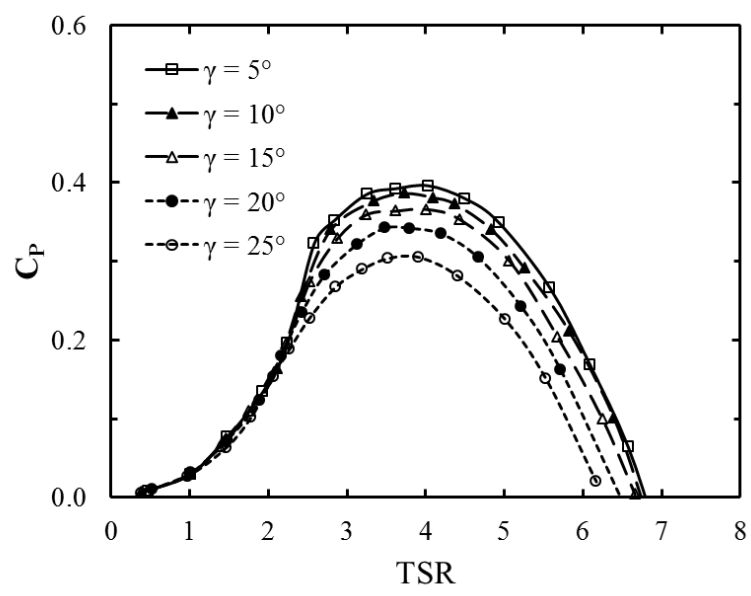

(c) $1.1 \mathrm{~m} / \mathrm{s}$ water velocity

Figure 6.5: Performance curves of the unshrouded model turbine in yaw operations. 
The reduction in the performance of an unshrouded turbine with increasing the yaw angle is well understood. The reason for the performance loss is that increasing the yaw angle changes the effective flow velocity component and the angles of attack on the blades which alters the aerodynamic characteristics of the blades. This results in a reduced lift force on the blades and consequently a reduced torque on the turbine shaft (Burton et al., 2001). A reduced torque on the shaft translates into a reduced output power of a turbine operating in yaw angles. In addition, the projected swept area of a turbine in a yawed position is smaller than in an inline operation. Therefore, the effective inlet area to the rotor blade facing the incoming flow is reduced for the turbine (Grant et al., 1997; Maeda et al., 2008). These are two main causes for the performance reduction of a turbine in yaw operations. A shroud that could eliminate these two root causes would be able to conserve the turbine performance in yaw operations. We next look into the capability of the shroud and the diffuser in moderating the effect of yaw angle on the turbine performance.

Figure 6.6 displays the performance curves of the model turbine with the shroud at the three Reynolds numbers of the experiments. The overlap of the low tip speed ratio portion of the curves due to the stall is also seen here. In contrast to the unshrouded turbine, the rest of the power curves for the turbine with the shroud overlap as well. It is observed that the shroud keeps the optimum and high tip speed ratio portions of the power curves nearly unchanged for the range of the yaw angles investigated. The maximum performance of the turbine in inline operations is preserved in yaw operations as the result of using the shroud. The maximum reduction in the performance of the turbine with the shroud compared to the inline operations is $8 \%$ occurring at $0.7 \mathrm{~m} / \mathrm{s}$ 
water velocity. The maximum reduction in 0.9 and $1.1 \mathrm{~m} / \mathrm{s}$ water velocities is $6 \%$ and $5 \%$, respectively. In addition to conserving the maximum performance, the shroud keeps the working range of the turbine unchanged: the power curves have the same runaway points at each Reynolds number. The shroud inlet is responsible for the invariant performance curves. The well-designed inlet of the shroud is capable of compensating the effect of yawed inflow in reducing the angles of attack and the projected swept area. An inlet contraction also reduces the inflow asymmetry which is responsible for degrading the pressure recovery of a diffuser (Klein, 1981). It should be noted that the maximum yaw angle examined in the experiments, $25^{\circ}$, is close to the inlet convergence angle of the shroud, $27^{\circ}$. The observed advantages of the shroud in yaw operations are however anticipated to alter in the yaw angles well larger than the inlet contraction angle.

Figure 6.7 shows the performance curves of the model turbine with the diffuser at the three Reynolds numbers of the experiments. The overlap of the low tip speed ratio section of the curves due to the stall seen for the unshrouded turbine and the turbine with the shroud is also observed here. The optimum tip speed ratio portion of the curves descends with increasing the yaw angle. The diffuser is not able to prevent the reduction of the turbine performance at the optimum portion of the power curve. However, the diffuser reduces the effect of yaw angle on the high tip speed ratio portion of the curves which results in the overlap of the curves in Figure 6.7. The runaway point of the turbine with the diffuser also stays nearly unchanged, i.e. the diffuser is able to keep the operating range of the turbine fixed in yaw operations. 


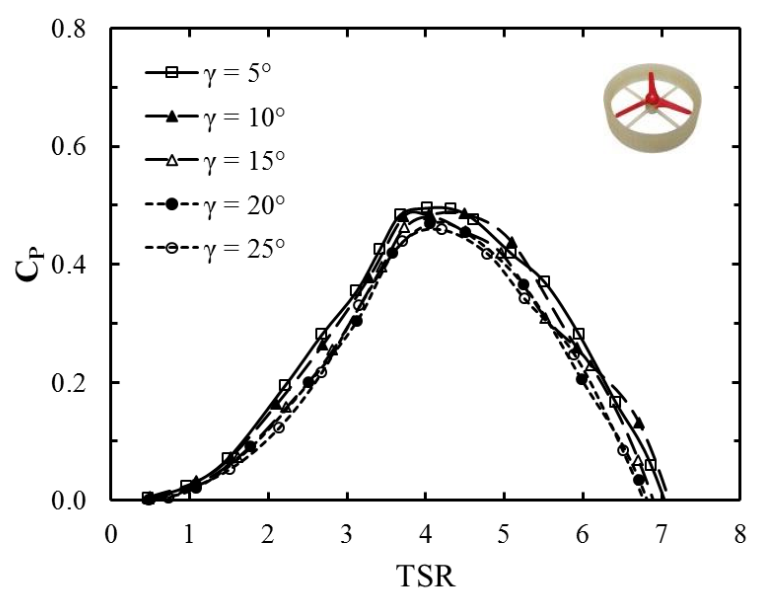

(a) $0.7 \mathrm{~m} / \mathrm{s}$ water velocity

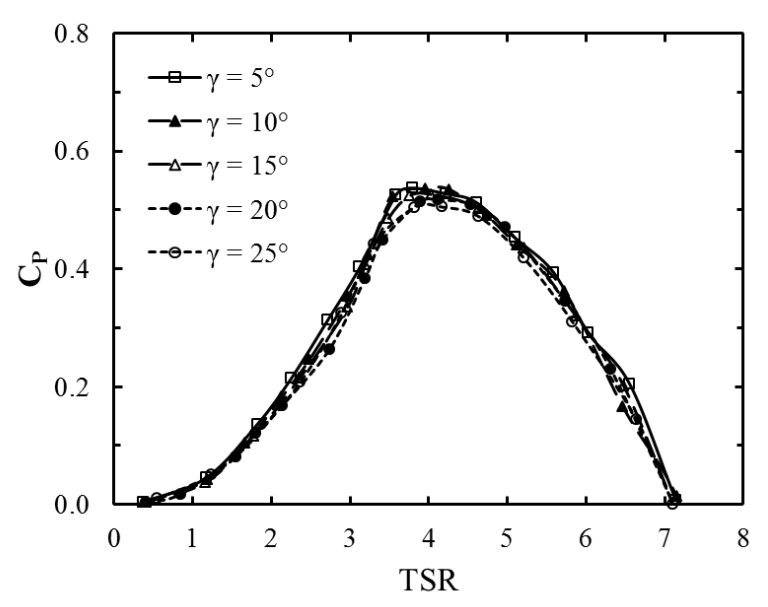

(b) $0.9 \mathrm{~m} / \mathrm{s}$ water velocity

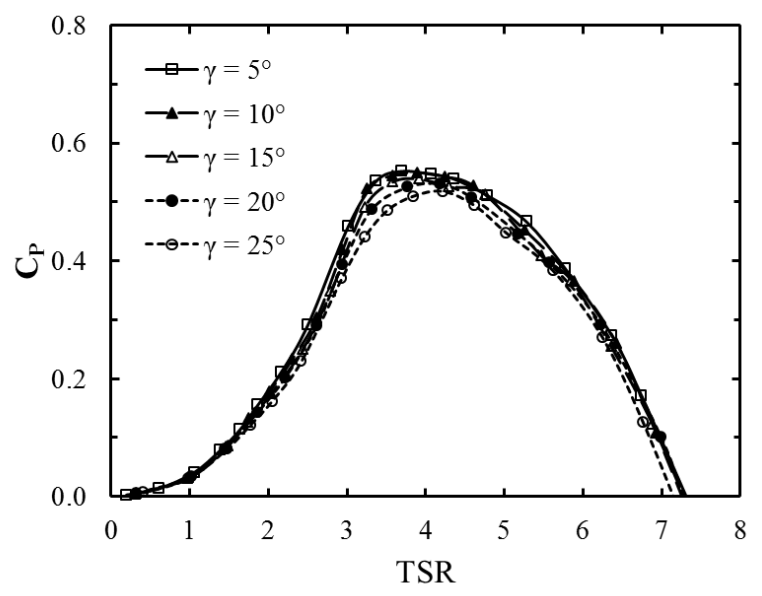

(c) $1.1 \mathrm{~m} / \mathrm{s}$ water velocity

Figure 6.6: Performance curves of the turbine with the shroud in yaw operations. 


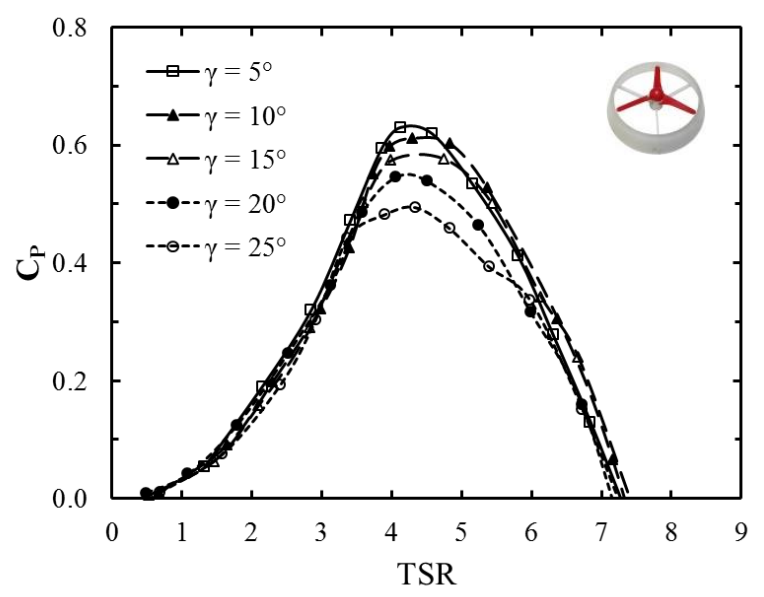

(a) $0.7 \mathrm{~m} / \mathrm{s}$ water velocity

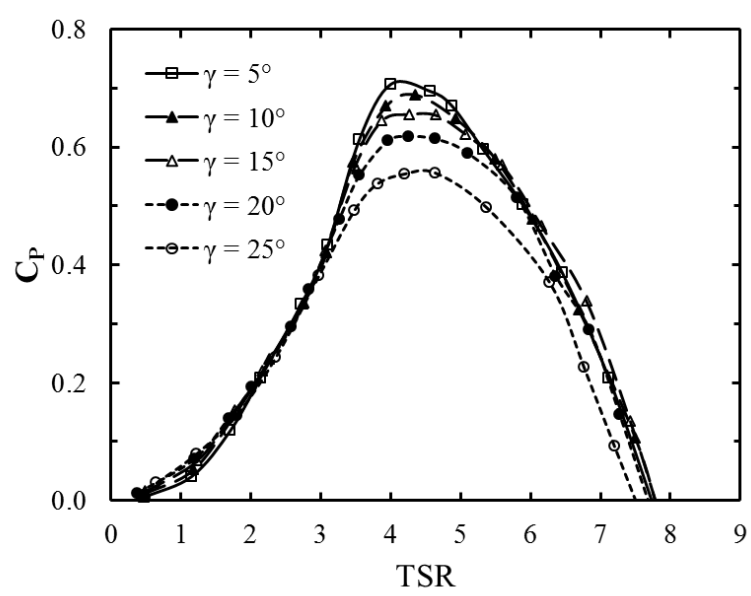

(b) $0.9 \mathrm{~m} / \mathrm{s}$ water velocity

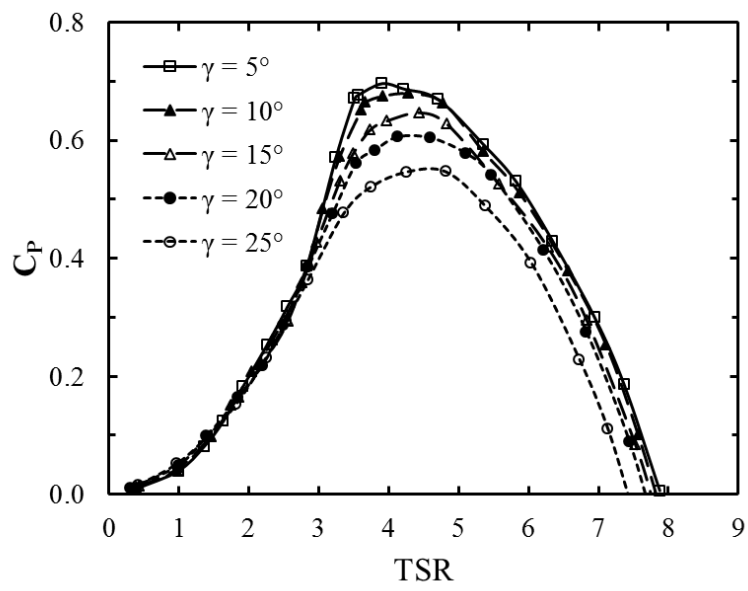

(c) $1.1 \mathrm{~m} / \mathrm{s}$ water velocity

Figure 6.7: Performance curves of the turbine with the diffuser in yaw operations. 
Careful examination of Figures 6.6 and 6.7 reveals the different functions of the shroud and the diffuser on the turbine performance in yaw operations. It is observed from the experimental results that the shroud affects both the optimum and high tip speed ratio portions of the performance curves. The diffuser influence is restricted to the high tip speed ratio portion of the curves. The shroud having convergent and divergent sections keeps the optimum and high tip speed ratio portions of the power curves unchanged. The diffuser having a divergent section keeps the high tip speed ratio part of the power curves invariant. It is therefore concluded the convergent section of a shroud, the inlet, affects the optimum tip speed ratio portion of the performance curve in yaw operations. The divergent section, the exit, is responsible for influencing the high tip speed ratio portion of the power curve and the runaway point. The conclusion is expected to be true for optimally designed shrouds with carefully selected convergence and divergence angles.

\subsection{Torque Coefficients}

Figures 6.8 to 6.10 show the torque coefficient curves for the shrouded and unshrouded model turbines in yaw operations. The torque coefficient is derived by dividing the power coefficient by the tip speed ratio. The principal use of a torque curve is for torque assessment of a gearbox and generator coupled to a turbine shaft (Burton et al., 2001). The experimental results show the torque coefficients of the model turbine in yaw operations decrease as the yaw angle increases. Like in inline operations, the maximum torque of a yawed turbine precedes the maximum power, i.e. the maximum torque happens in a lower tip speed ratio than the maximum power (Krogstad and Adaramola, 2012). 


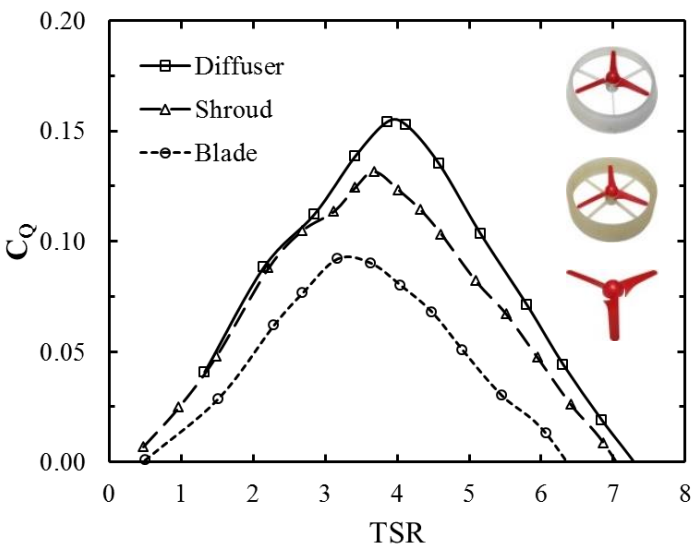

(a) $\gamma=5^{\circ}$

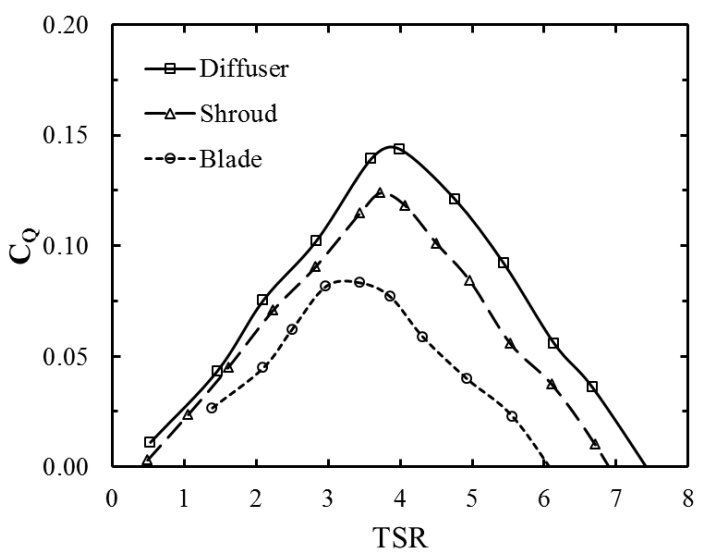

(c) $\gamma=15^{\circ}$

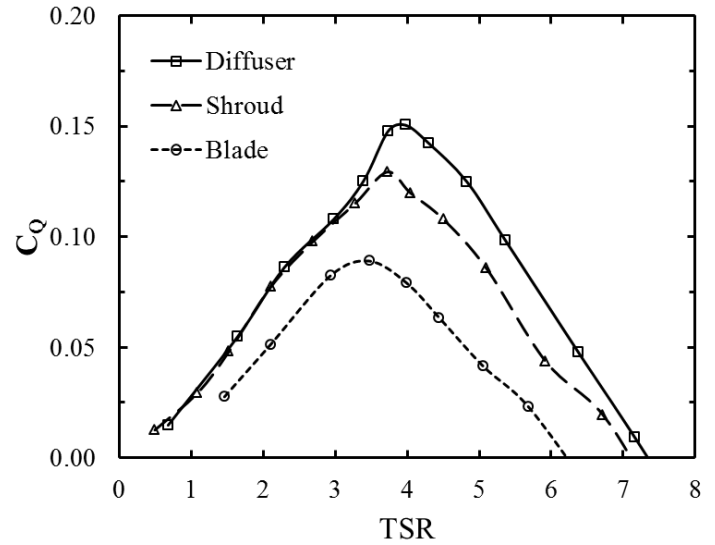

(b) $\gamma=10^{\circ}$

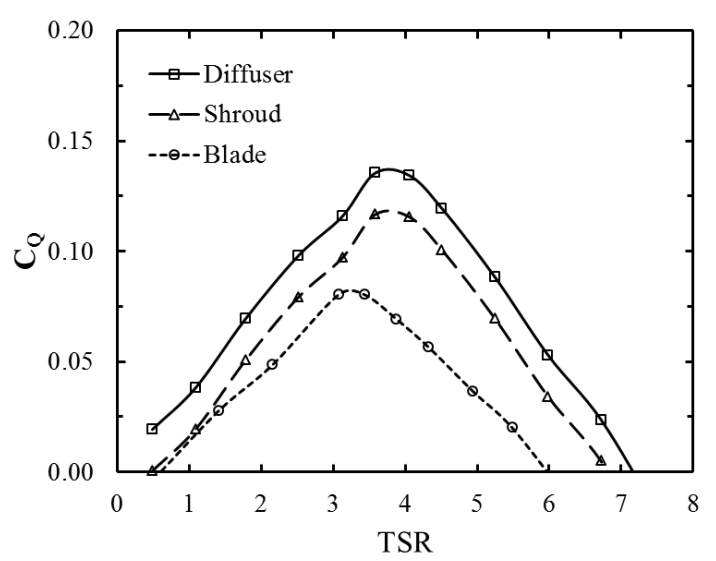

(d) $\gamma=20^{\circ}$

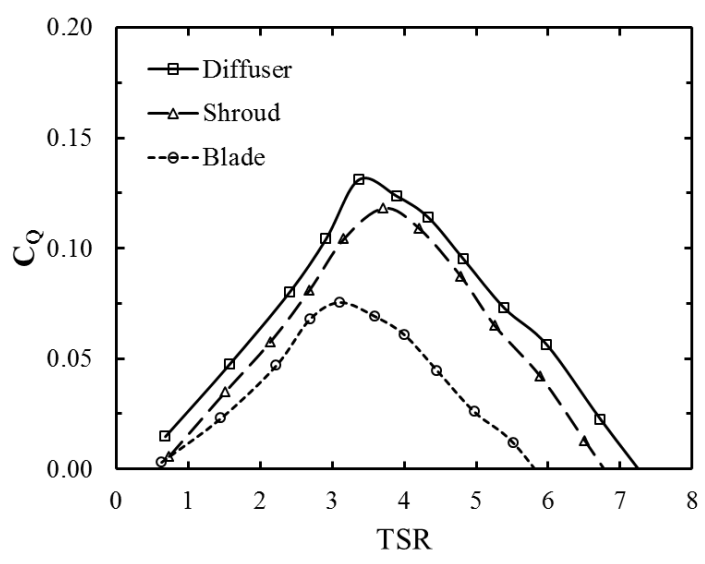

(e) $\gamma=25^{\circ}$

Figure 6.8: Torque coefficients of the model turbine in yaw operations at $0.7 \mathrm{~m} / \mathrm{s}$ water velocity. 


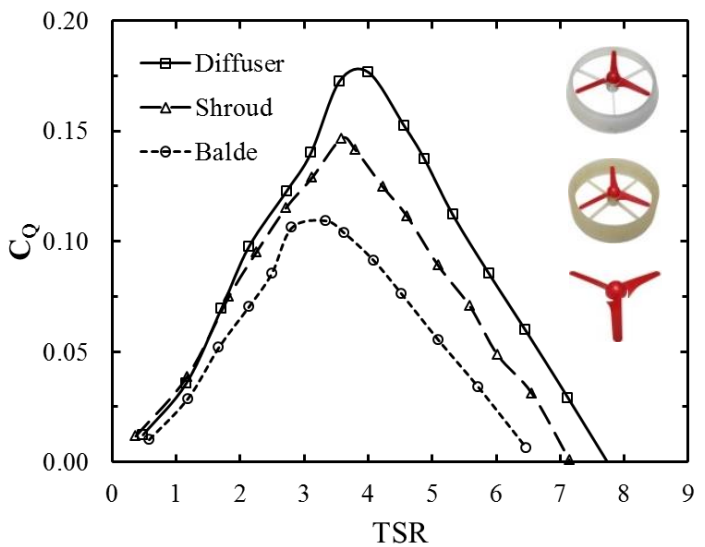

(a) $\gamma=5^{\circ}$

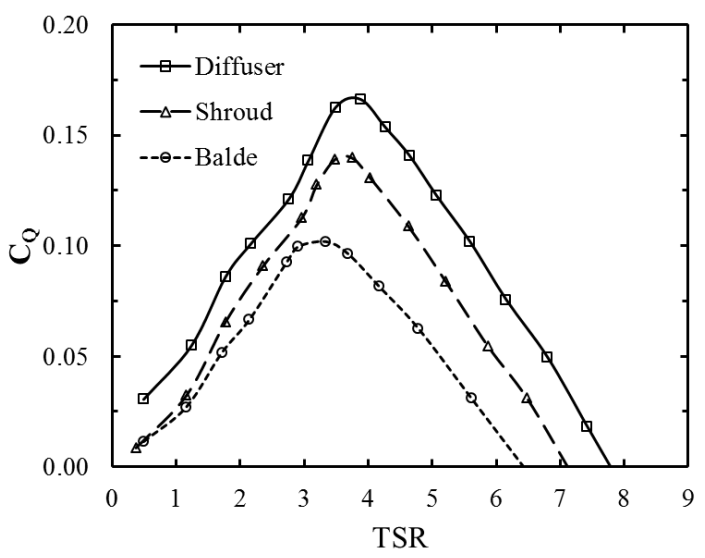

(c) $\gamma=15^{\circ}$

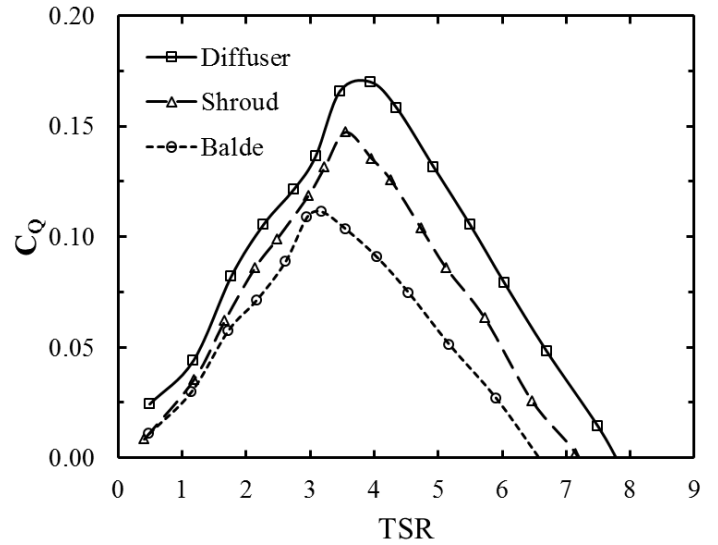

(b) $\gamma=10^{\circ}$

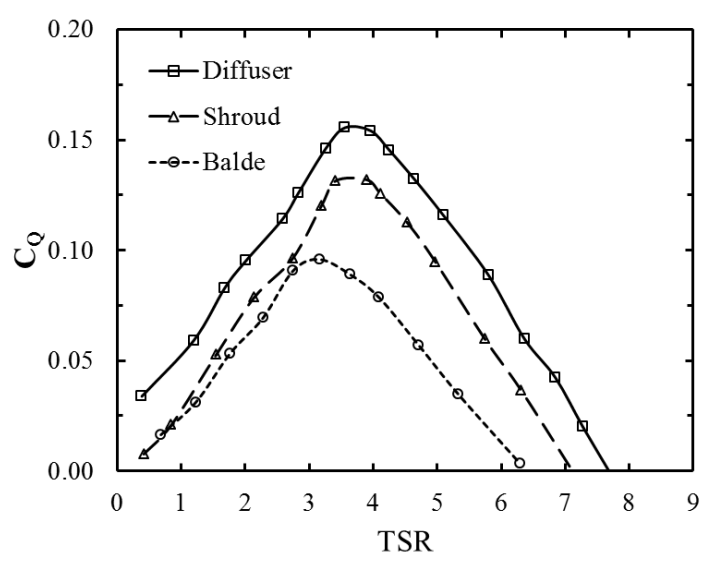

(d) $\gamma=20^{\circ}$

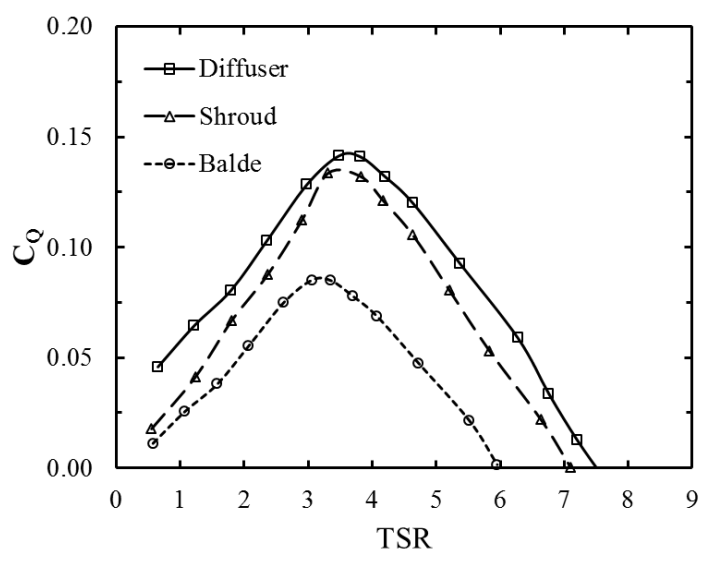

(e) $\gamma=25^{\circ}$

Figure 6.9: Torque coefficients of the model turbine in yaw operations at $0.9 \mathrm{~m} / \mathrm{s}$ water velocity. 


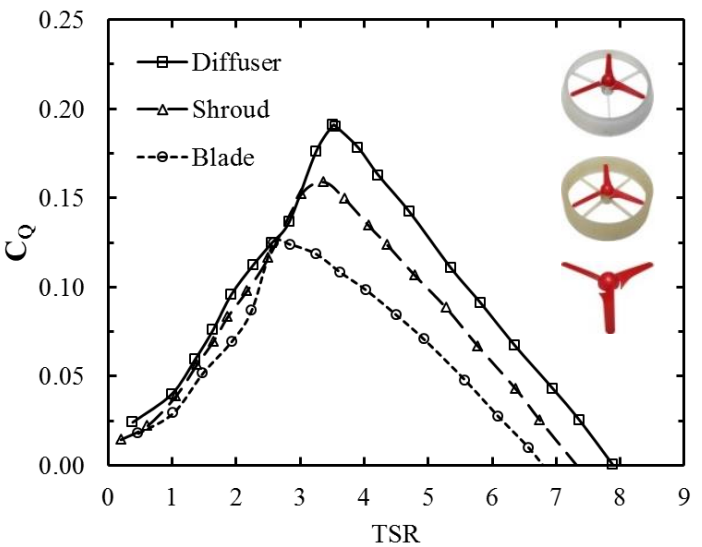

(a) $\gamma=5^{\circ}$

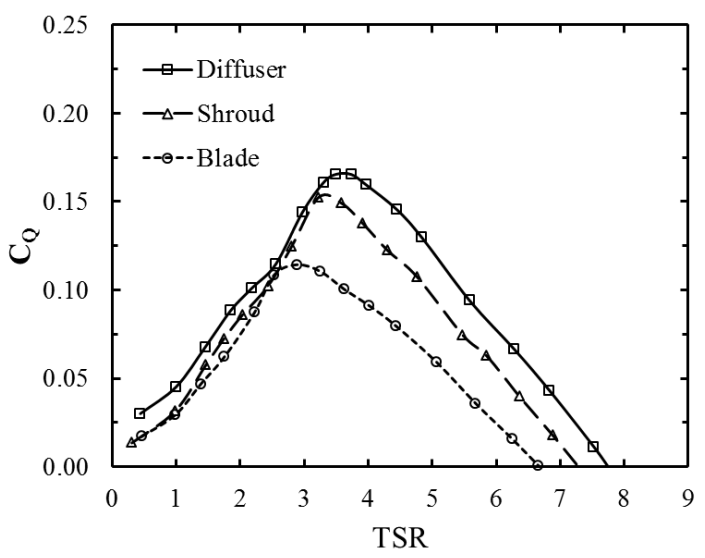

(c) $\gamma=15^{\circ}$

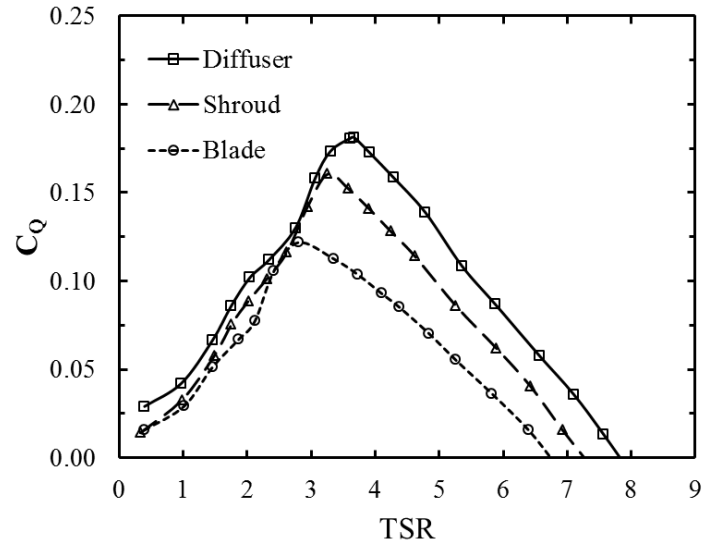

(b) $\gamma=10^{\circ}$

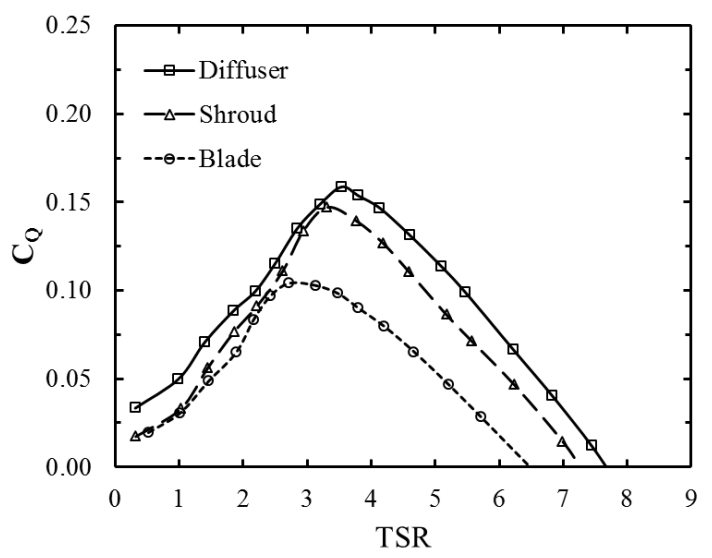

(d) $\gamma=20^{\circ}$

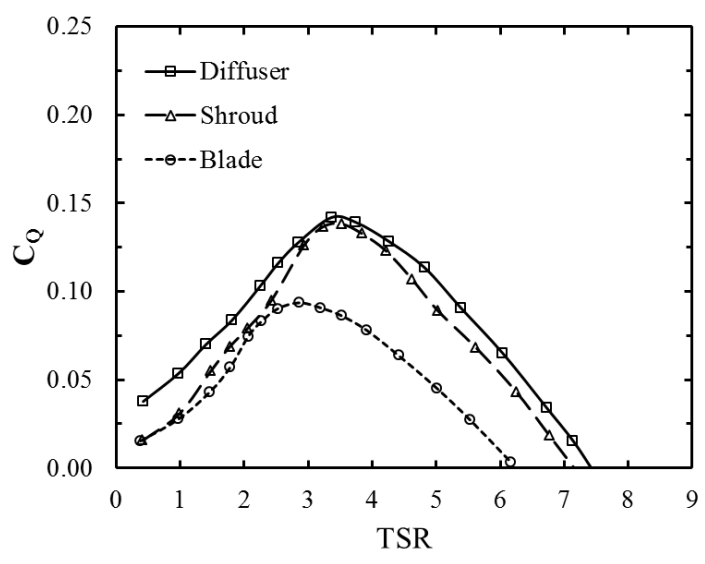

(e) $\gamma=25^{\circ}$

Figure 6.10: Torque coefficients of the model turbine in yaw operations at $1.1 \mathrm{~m} / \mathrm{s}$ water velocity. 


\subsection{Thrust Coefficients}

Figures 6.11 to 6.13 show the thrust coefficients of the shrouded and unshrouded model turbines in yaw operations. The coefficients are calculated based on the blade swept area and are corrected for the free surface proximity and blockage effects. Similar to the power and torque coefficients, the thrust coefficient decreases in yaw operations. The reduction in the thrust coefficient is small for yaw angles up to $10^{\circ}$ but increases as the yaw angle increases. The skewness of the turbine wake as well as the reduction in the projected rotor area and the effective flow velocity result in the reduction of the thrust coefficients (Krogstad and Adaramola, 2012). The thrust force on a turbine rotor is directly transferred to the turbine structure and affects the design of the structure and anchoring system (Burton et al., 2001). 


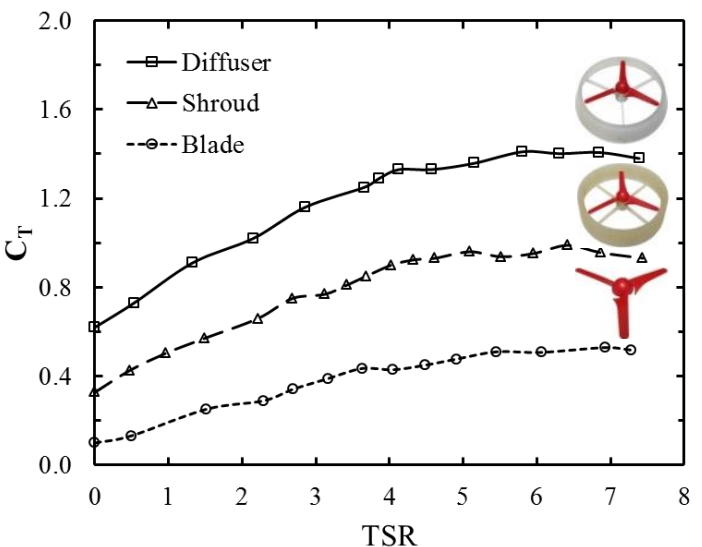

(a) $\gamma=5^{\circ}$

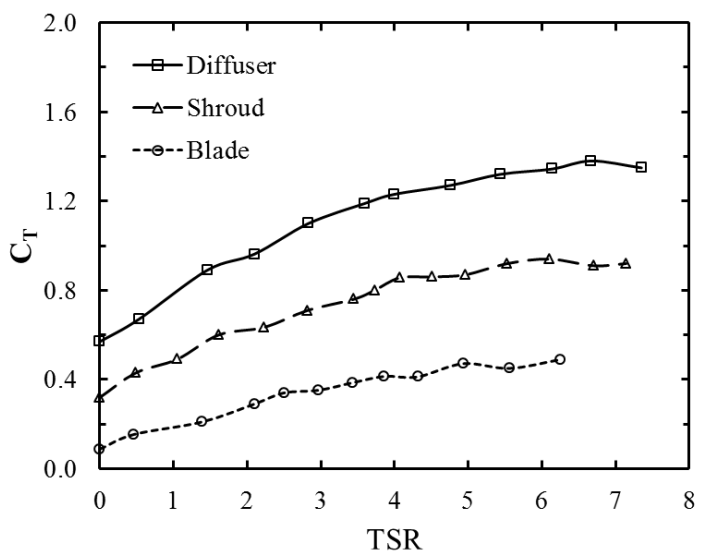

(c) $\gamma=15^{\circ}$

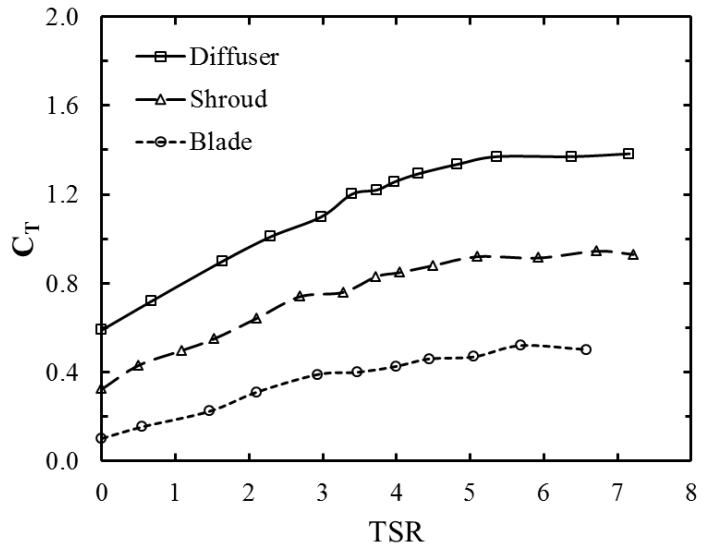

(b) $\gamma=10^{\circ}$

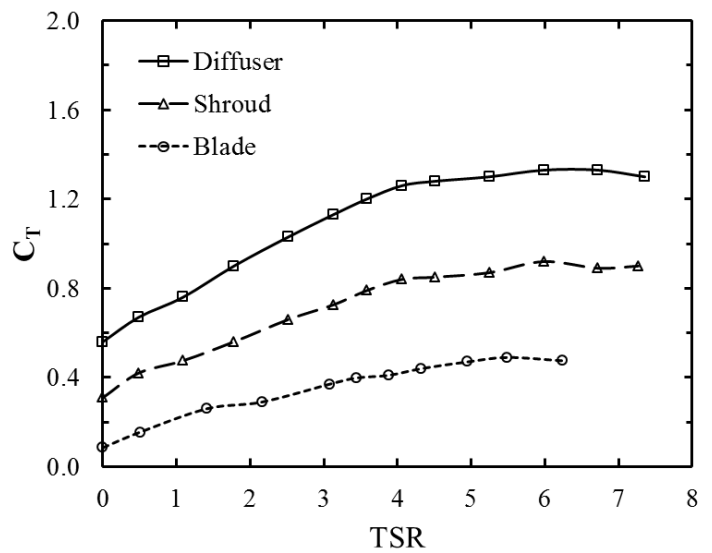

(d) $\gamma=20^{\circ}$

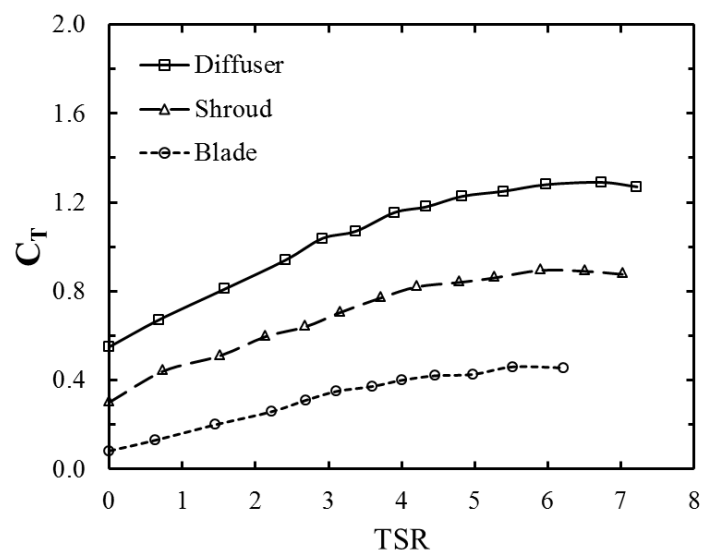

(e) $\gamma=25^{\circ}$

Figure 6.11: Thrust coefficients of the model turbine in yaw operations at $0.7 \mathrm{~m} / \mathrm{s}$ water velocity. 


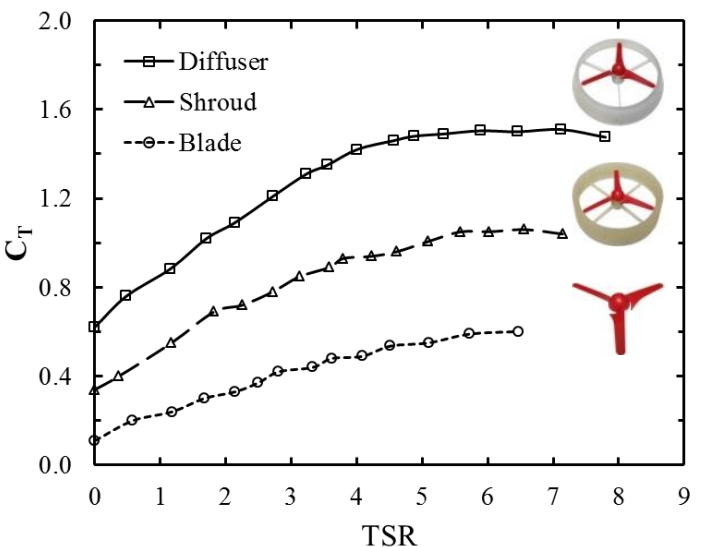

(a) $\gamma=5^{\circ}$

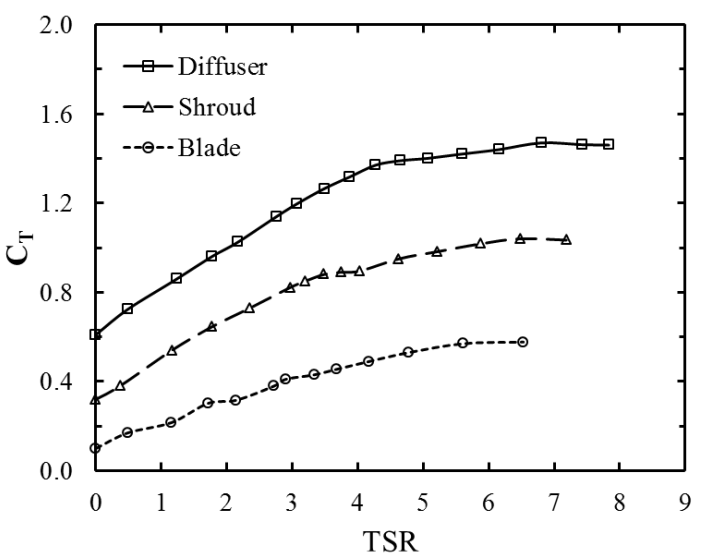

(c) $\gamma=15^{\circ}$

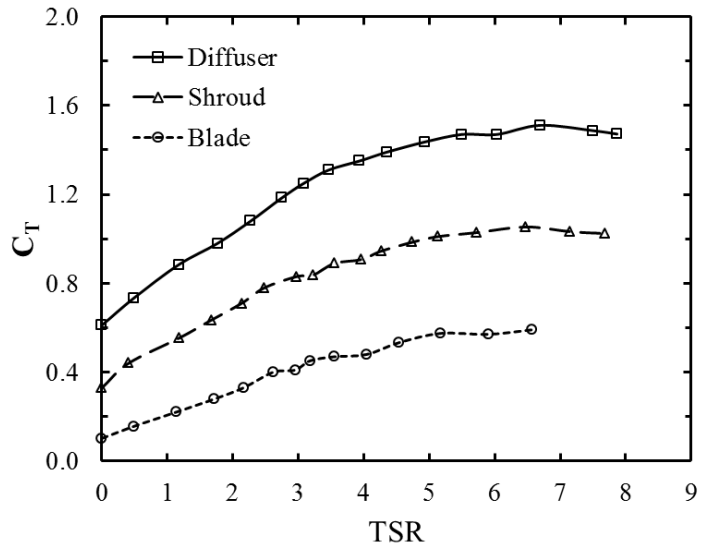

(b) $\gamma=10^{\circ}$

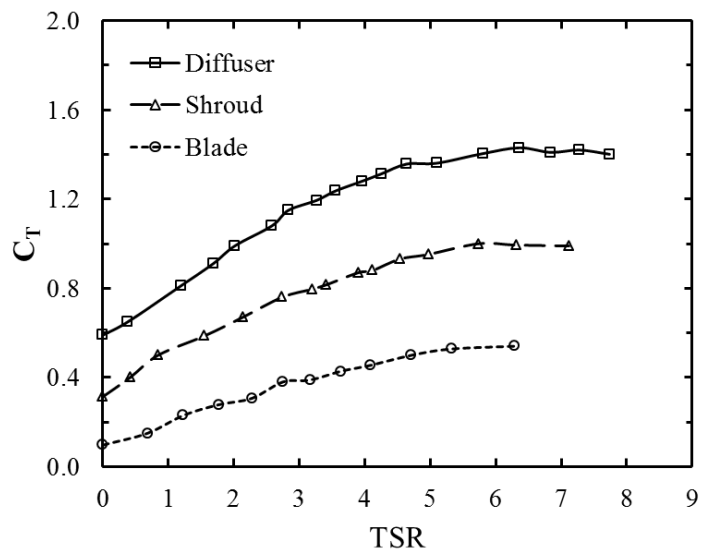

(d) $\gamma=20^{\circ}$

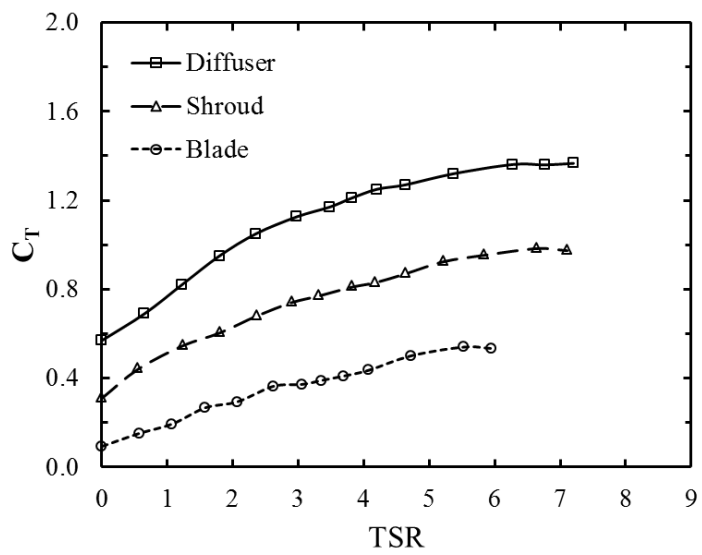

(e) $\gamma=25^{\circ}$

Figure 6.12: Thrust coefficients of the model turbine in yaw operations at $0.9 \mathrm{~m} / \mathrm{s}$ water velocity. 


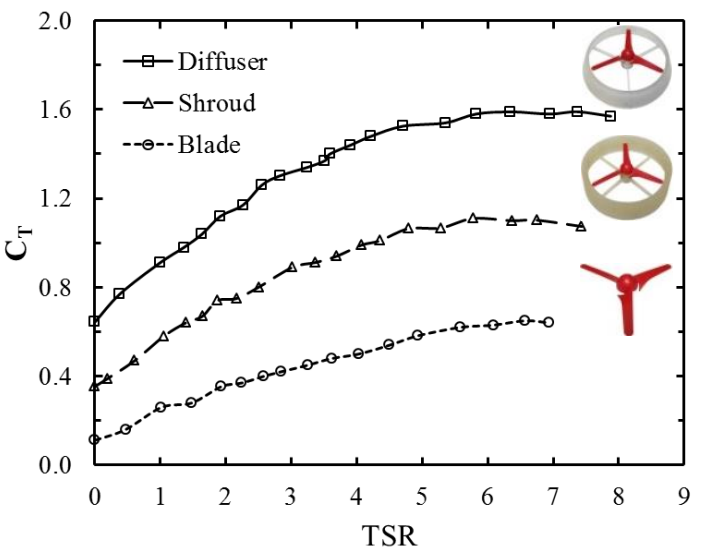

(a) $\gamma=5^{\circ}$

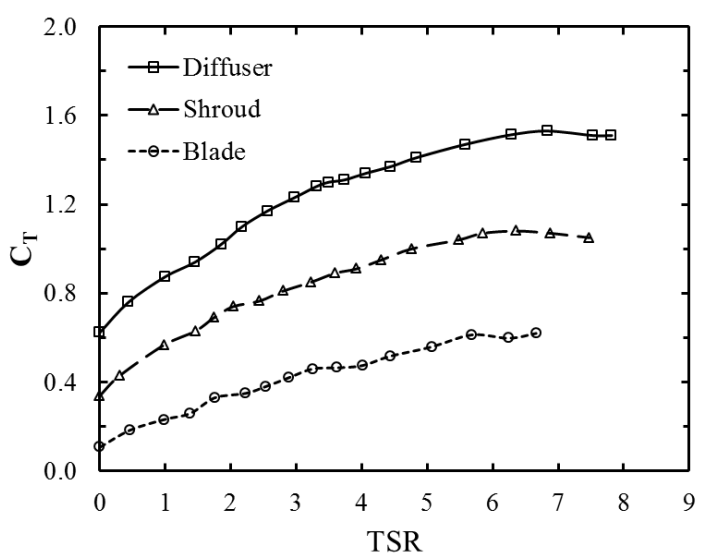

(c) $\gamma=15^{\circ}$

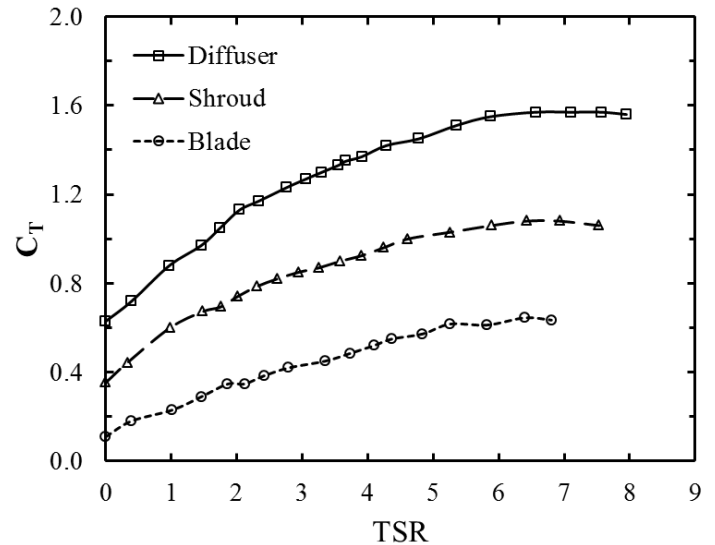

(b) $\gamma=10^{\circ}$

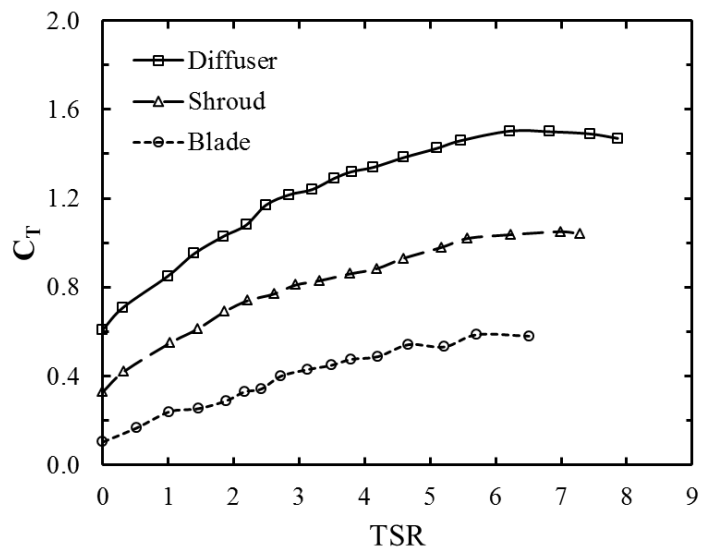

(d) $\gamma=20^{\circ}$

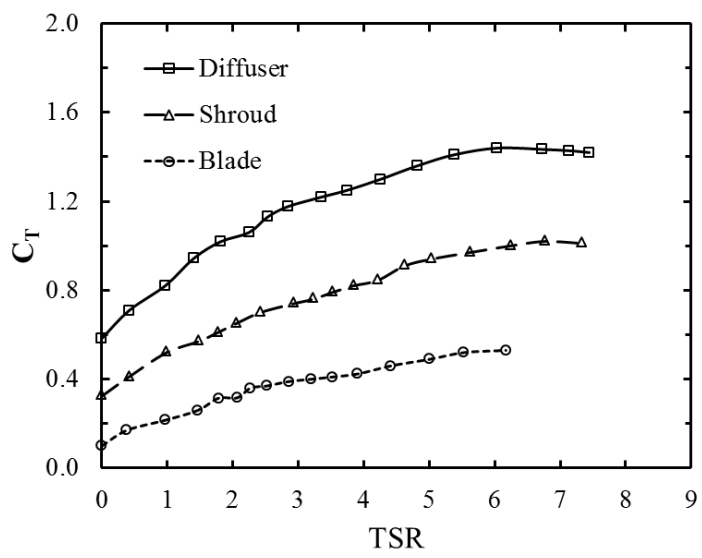

(e) $\gamma=25^{\circ}$

Figure 6.13: Thrust coefficients of the model turbine in yaw operations at $1.1 \mathrm{~m} / \mathrm{s}$ water velocity. 


\subsection{Comparison to the Linear Momentum Theory}

\subsubsection{Wind Turbines Experimental Data}

In reviewing the theory of hydrokinetic turbines in Chapter 3 it is seen that the linear momentum theory uses simplified assumptions to predict the performance of an unshrouded turbine. In Section 3.3, the analysis is extended to a yawed turbine where the theory results in the cosine functions of the yaw angle for the power and thrust of a turbine. The maximum power of a hydrokinetic turbine and its thrust at the maximum power are theoretically expected to decrease with cosine cubed and cosine squared of the yaw angle, respectively. The momentum theory uses ideal assumptions some of which are not applicable to a turbine in yaw operations (Burton et al., 2001). Therefore, experimental verifications are needed if the theoretical results are to be used with confidence in assessing the performance of a yawed turbine or as control parameters in optimizing the output power of a turbine farm. Figures 6.14 and 6.15 compare experimental power and thrust of unshrouded wind turbines in yaw operations available in the literature with the cosine cubed and cosine squared rules of linear momentum theory. Figure 6.14 shows the ratio of the maximum power in yaw angle to the maximum inline power. A close agreement is seen between the experimental data and the line of cosine cubed of the yaw angle. A maximum discrepancy of $10 \%$ is seen between the experimental results of Anderson et al. (1982) at $30^{\circ}$ yaw angle and the cosine cubed

rule. The difference between the experimental and theoretical results is less than $10 \%$ for the other studies and yaw angles. Figure 6.15 shows the ratio of the thrust at the maximum power in yaw angles to the thrust at the maximum power in the inline 
operation. Here also a close agreement is seen between the experimental data and the line of cosine squared of the yaw angle. A maximum discrepancy in the thrust curve happens at $30^{\circ}$ yaw angle for the experimental results of National Renewable Energy Laboratory, (Hand et al., 2001). The maximum difference is 5\% and the discrepancy between the experimental and theoretical results is less than $5 \%$ for the other studies and yaw angles. It is therefore concluded the cosine cubed and cosine squared rules of the linear momentum theory are in close agreement with experimental results. The cosine rules can be used with confidence in evaluating the performance of an unshrouded kinetic turbine in yaw operations. The experimental results for the shrouded and unshrouded model turbines need to be compared with the results of the momentum theory to try finding similar relations for shrouded turbines.

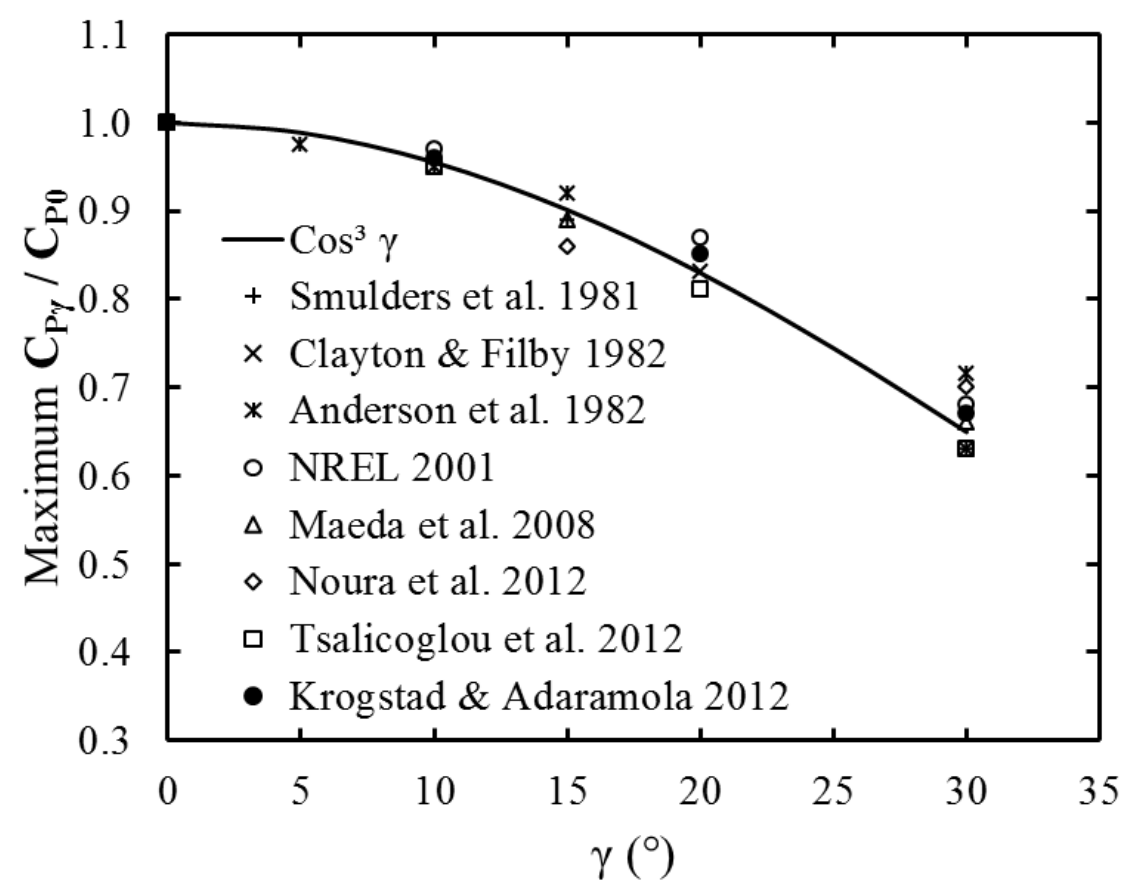

Figure 6.14: Comparison between the experimental power reduction of wind turbines in yaw angles and the cosine cubed rule of the linear momentum theory. 


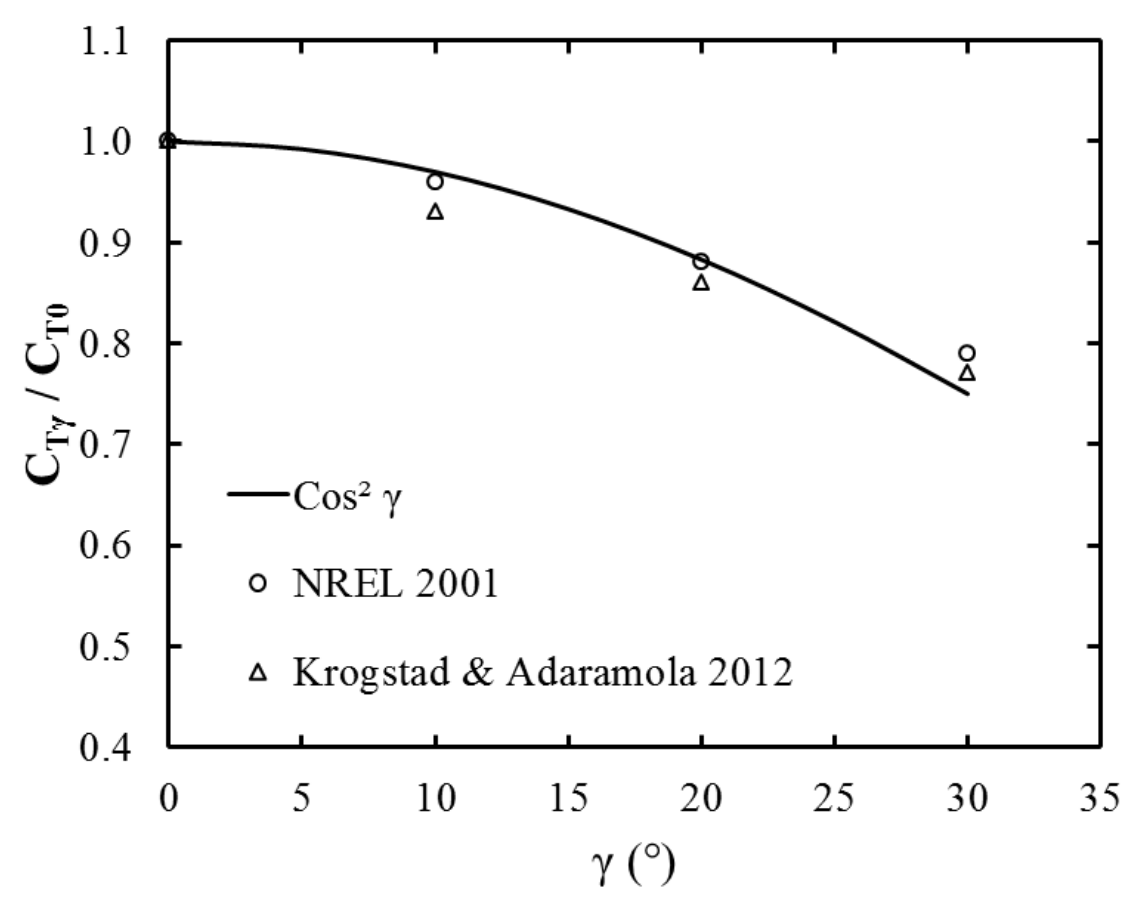

Figure 6.15: Comparison between the experimental reduction in the thrust at the maximum power of wind turbines in yaw angles and the cosine squared rule of the linear momentum theory.

\subsubsection{The Unshrouded Model Turbine}

It is shown so far that the linear momentum theory accurately predicts the decreasing behaviour of the power and thrust coefficients of a hydrokinetic turbine in yaw operations. It is of interest to have such basic relations to predict the performance reduction of a shrouded hydrokinetic turbine in yaw operations. No such relations are available in the literature for shrouded turbines and having that would be useful to marine renewable energy industry. We first compare the power and thrust coefficients of our unshrouded model turbine to the results of the linear momentum theory. Figure 6.16 depicts the ratio of the maximum power of the blade at yaw angles to the maximum inline power. It is observed the experimental data follows the line of cosine cubed of the yaw angle. The maximum difference between the experimental data and the cosine cubed 
line is $4.1 \%$ which occurs at $25^{\circ}$ yaw angle. Everywhere else in the diagram, the discrepancy is less than $4.1 \%$. Figure 6.17 shows the ratio of the thrust at the maximum power at yaw angles to the thrust at the maximum power in zero yaw. The experimental data closely follows the line of cosine squared of the yaw angle with a maximum difference of $4.0 \%$ occurred at $25^{\circ}$ yaw angle. For the rest of the data, the discrepancy remains less than $4 \%$. Our results further confirm the results of the previous experimental studies for unshrouded turbines that the linear momentum theory can precisely predict the decreasing behaviour of the power and thrust coefficients of a hydrokinetic turbine. The rules of cosine cubed and cosine squared of the yaw angle are adequately precise to predict the loss in the performance of hydrokinetic turbines in yaw operations.

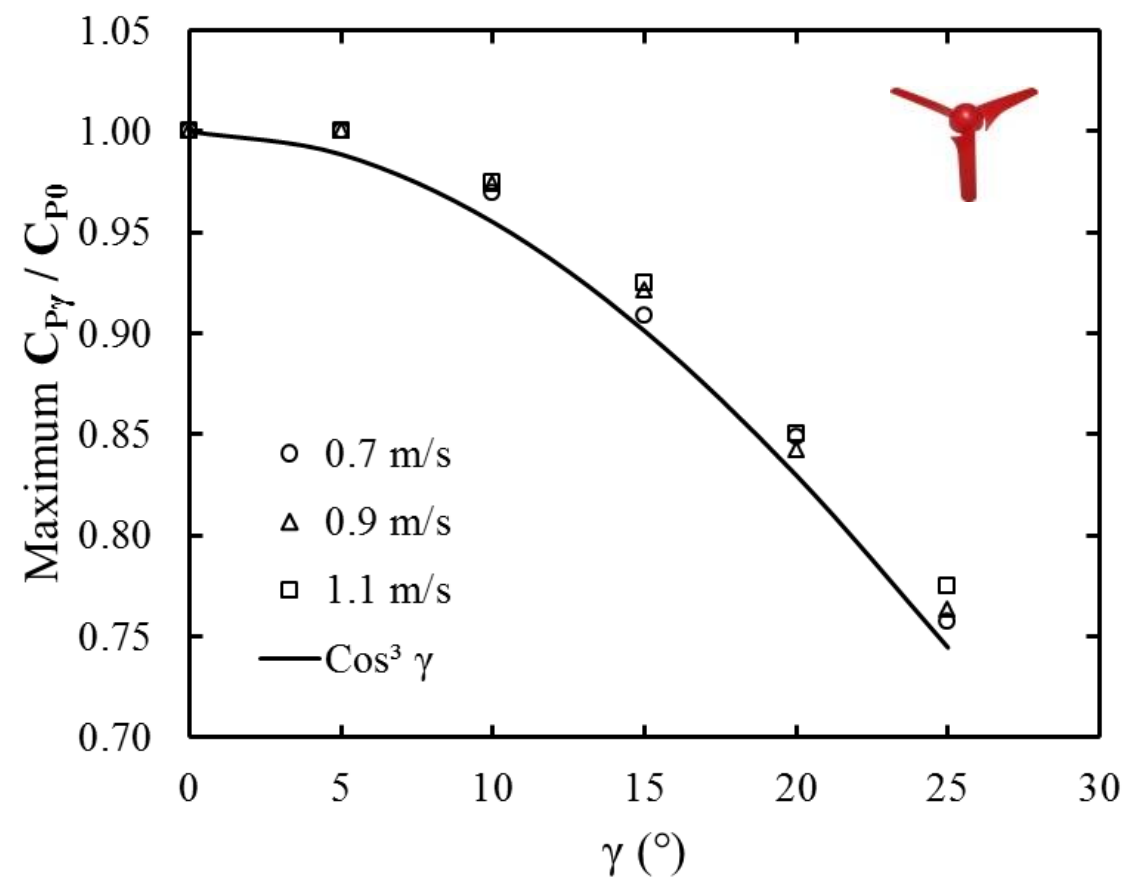

Figure 6.16: Reduction in the maximum power coefficients of the unshrouded model turbine at yaw angles. 


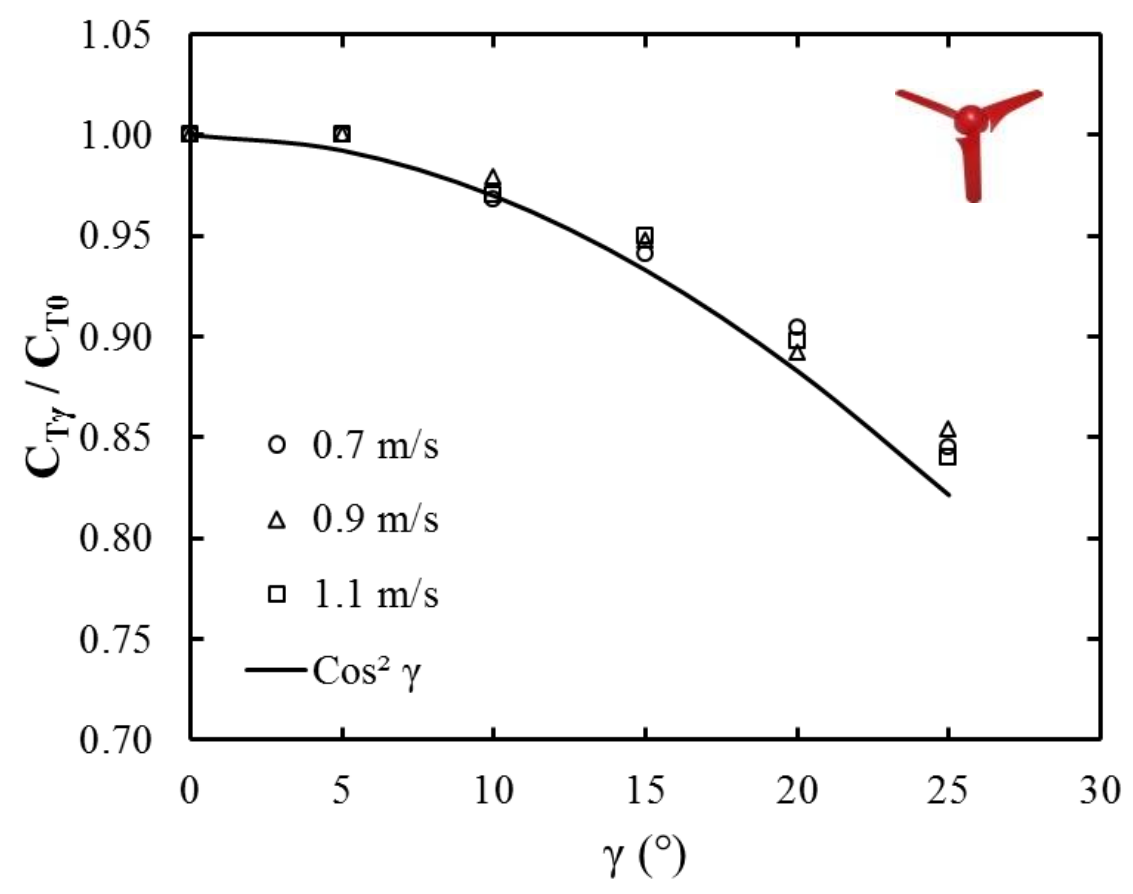

Figure 6.17: Reduction in the thrust coefficients at the maximum power of the unshrouded model turbine at yaw angles.

\subsubsection{The Model Turbine with the Shroud}

After comparing the power and thrust coefficients of the blade to the linear momentum theory, it is now possible to examine the reduction in the power and thrust of the model turbine with the shroud. Figure 6.18 shows the ratio of the maximum power of the model turbine with the shroud at yaw angles to the maximum inline power. The lines of cosine and cosine cubed of the yaw angle are also graphed and it is observed that the experimental data closely follows the line of cosine of the yaw angle. The maximum difference between the experimental data and the cosine line is $3.5 \%$ and occurs at $25^{\circ}$ yaw angle. The discrepancy remains less than $3.5 \%$ everywhere else in the diagram. It is seen that the shroud effectively reduces the turbine power reduction from cosine cubed to cosine of the yaw angle. Figure 6.19 shows the ratio of the thrust at the maximum power at yaw angles to the thrust at the maximum power in the inline operations. The lines of 
cosine and cosine squared of the yaw angle are also graphed and the experimental data is observed to narrowly follow the line of cosine of the yaw angle. The maximum discrepancy between the experimental data and the cosine line is $1.5 \%$ occurred at $25^{\circ}$ yaw angle. The discrepancy is less than $1.5 \%$ for the rest of the data points. The shroud decreases the reduction in the thrust of the model turbine from cosine squared to cosine of the yaw angle. Therefore, it is concluded the shroud effectively reduces the sensitivity of the model turbine performance to changes in the yaw angle. Based on the cosine rule found in this study, the turbine with the shroud in a $45^{\circ}$ yawed flow generates $29 \%$ less power than in an inline flow. The unshrouded turbine, based on the cosine cubed rule, would produce $65 \%$ less power in the same flow.

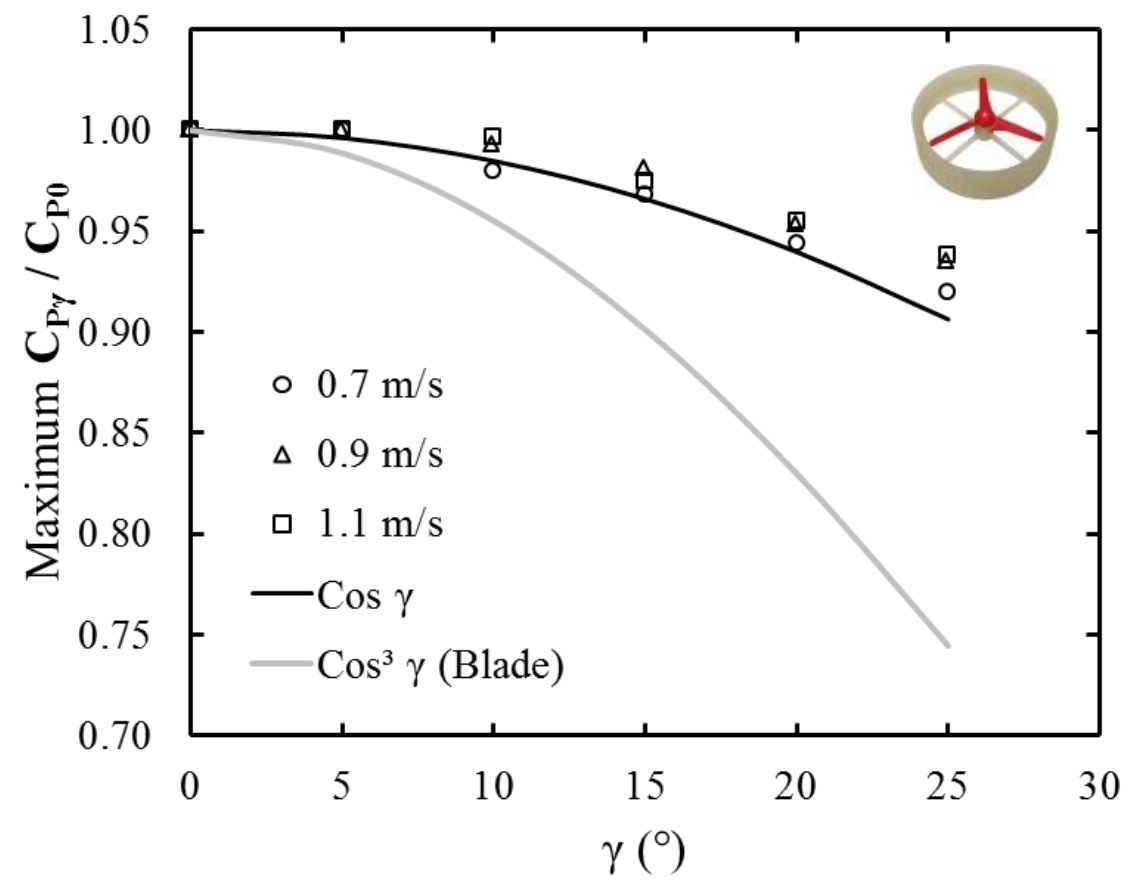

Figure 6.18: Reduction in the maximum power coefficients of the model turbine with the shroud at yaw angles. 


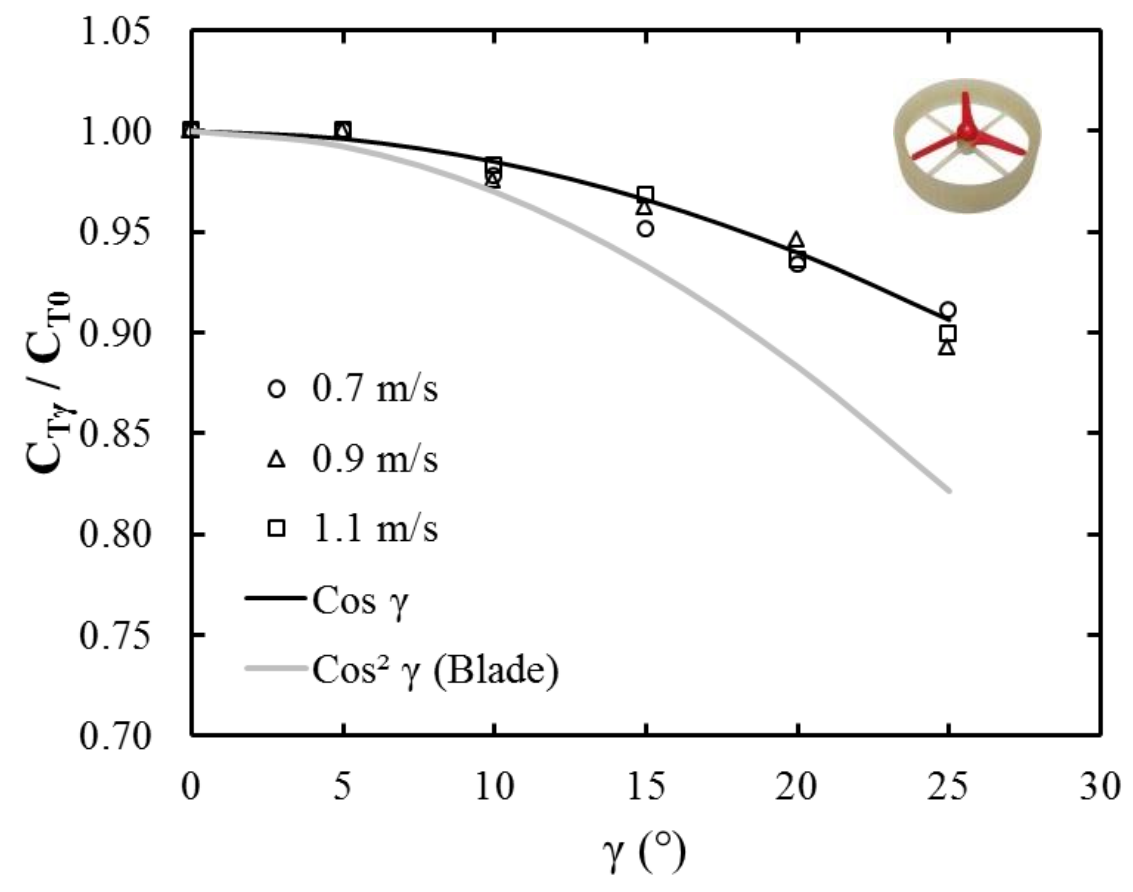

Figure 6.19: Reduction in the thrust coefficients at the maximum power of the model turbine with the shroud at yaw angles.

\subsubsection{The Model Turbine with the Diffuser}

We now examine the reduction in the power and thrust coefficients of the model turbine with the diffuser and compare them to the cosine rules of the blade and the shroud. Figure 6.20 shows the ratio of the maximum power of the model turbine with the diffuser at yaw angles to the maximum inline power. The lines of cosine, cosine squared, and cosine cubed of the yaw angle are also shown. It is observed the experimental data is well predicted by the line of cosine squared of the yaw angle. The maximum difference between the experimental data and the line of cosine squared is $5.3 \%$ and occurs at $25^{\circ}$ yaw angle. The discrepancy remains less than 5.3\% everywhere else in the diagram. It is seen the diffuser reduces the turbine power reduction from cosine cubed to cosine squared of the yaw angle. Figure 6.21 shows the ratio of the thrust at the maximum power at yaw angles to the thrust at the maximum power in zero yaw. The lines of cosine 
and cosine squared of yaw angle are also graphed and the experimental data is observed to narrowly follow the line of cosine of the yaw angle. The maximum discrepancy between the experimental data and the cosine line is $2.1 \%$ occurred at $25^{\circ}$ yaw angle. The discrepancy is less than $2.1 \%$ for the rest of the data points. The diffuser decreases the reduction in the thrust of the model turbine from cosine squared to cosine of the yaw angle. The diffuser compared to the shroud is less effective in reducing the sensitivity of the model turbine power to the yaw angle. Based on the cosine squared rule found in this study, the turbine with the diffuser in a $45^{\circ}$ yawed flow generates $50 \%$ less power than in an inline flow. The unshrouded turbine and the turbine with the shroud would produce $65 \%$ and $29 \%$ less power in the same flow, respectively.

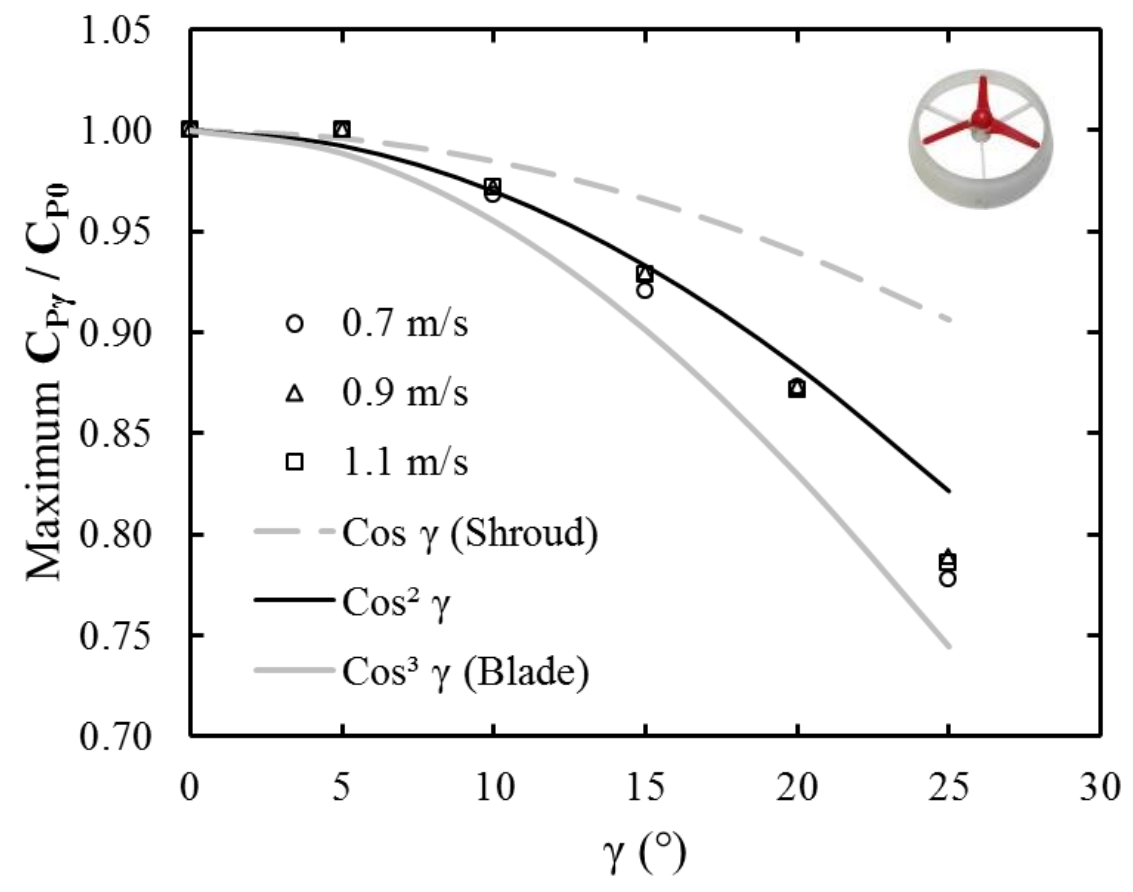

Figure 6.20: Reduction in the maximum power coefficients of the model turbine with the diffuser at yaw angles. 


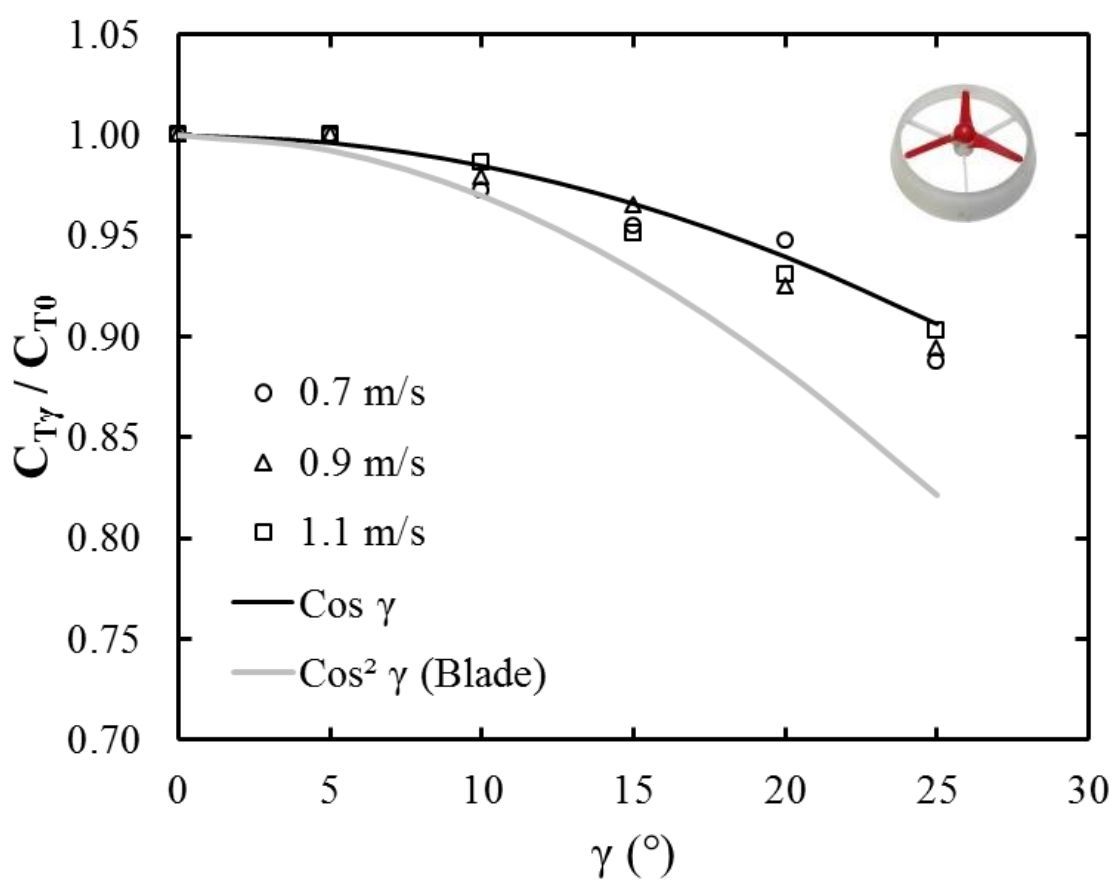

Figure 6.21: Reduction in the thrust coefficients at the maximum power of the model turbine with the diffuser at yaw angles.

\subsection{Verification of the Cosine Rules for Shrouded Turbines}

The cosine rules for the shrouded turbines deduced from the experimental results of this study has no record in the literature and are introduced here for the first time. The experimental studies available in the literature on shrouded turbines suffer from excessive tunnel blockage which resulted in artificially elevated power coefficients in yaw operations (Phillips, 2003). This renders the results of such studies unreliable for validation purposes. The only reliable study for validation is the experimental study by Phillips et al. (1999) performed on a diffuser augmented wind turbine in a wind tunnel. The shroud used in the experiment is a duct comparable to the shroud of the present study with an optimized airfoil shaped converging-diverging cross section. Figure 6.22 shows the ratio of the maximum power of the diffuser augmented wind turbine at yaw 
angles to the maximum inline power. The line of cosine of yaw angle is graphed and the experimental data is observed to be in a close agreement with it. The maximum discrepancy between the experimental data and the cosine line is $5.7 \%$ at $30^{\circ}$ yaw angle and the inconsistency is less than $5.7 \%$ everywhere else in the diagram. This can be considered as a verification to the cosine rule proposed for the shrouded model turbine since the shrouds used in the two studies are relatively similar having convergentdivergent cross sections. It is therefore concluded the cosine rules introduced in this study remain valid for any similar optimally designed shrouded turbine in the range of the yaw angles investigated. Validity of the cosine rules should be examined at larger yaw angles as the maximum yaw angle tested in this study is close to the half angles of the inlet and the outlet of the shrouds.

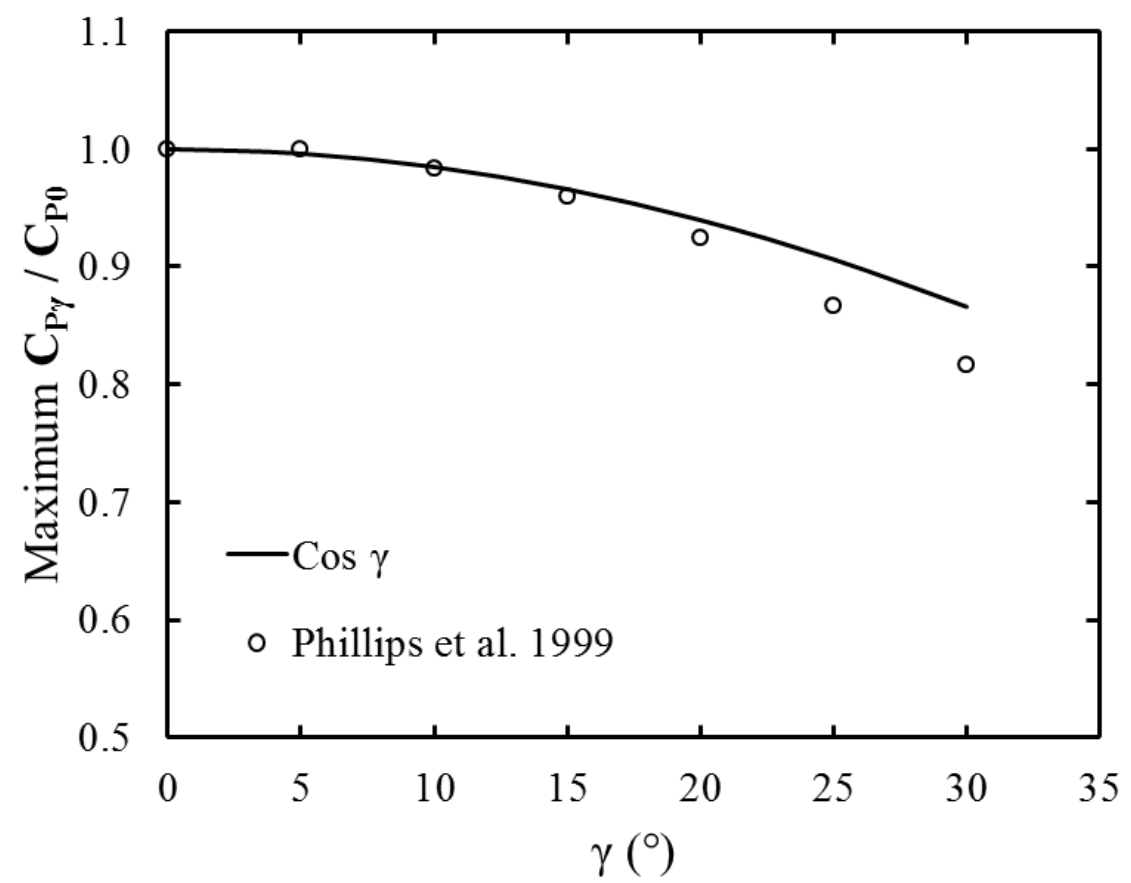

Figure 6.22: Comparison between the experimental power reduction of a diffuser augmented wind turbine and the rule of cosine of the yaw angle deduced in this study. 


\subsection{Velocity at the Rotor Plane}

Power of an ideal hydrokinetic turbine in the theoretical studies is calculated by multiplying the thrust of the turbine and the flow velocity at the rotor disk. Having the cosine rules for the power and thrust of the model turbine in yaw operations, we can deduce the changes in the flow velocity at the rotor plane. Table 6.4 summarizes the cosine rules for the shrouded and unshrouded turbines in yaw operations. For the blade we know from the momentum theory that the power and thrust are proportional to cosine cubed and cosine squared of the yaw angle. The flow velocity at the rotor plane is then deduced to be proportional to cosine of the yaw angle. This is obvious, as the momentum theory starts deriving the yaw performance of a turbine by using the normal component of velocity to the rotor disk, the cosine component. The power and thrust of the model turbine with the diffuser are proportional to cosine squared and cosine of the yaw angle, respectively. The flow velocity at the rotor plane is therefore deduced to be proportional to cosine of the yaw angle like for the unshrouded turbine. The effect of the diffuser on the power of the model turbine is therefore due to the influence it has on the thrust force and not on the velocity. The power and thrust of the model turbine with the shroud are proportional to cosine of the yaw angle. The flow velocity at the rotor plane is hence deduced to remain unchanged compared to an inline flow operation. Comparing the shroud and the diffuser, we observe the additional effect of the shroud on the power is due to the influence it has on the velocity at the rotor plane. The advantage of the shroud over the diffuser is the ability of its inlet in eliminating the effect of yaw angle on the flow velocity at the rotor plane. Inlet of the shroud with its optimum convergent design bends the flow streamlines and keeps them normal to the rotor plane. Therefore, the 
velocity vectors and not just the cosine components of the velocity remain normal to the rotor plane like for an inline flow. This characteristic of the shroud is expected to be a function of the yaw angle and is anticipated to alter in the higher angles as its inlet angle is close to the maximum yaw angle investigated in the experiments.

Table 6.4: The cosine rules for the performance reduction in yaw operations and the flow velocity at the rotor plane

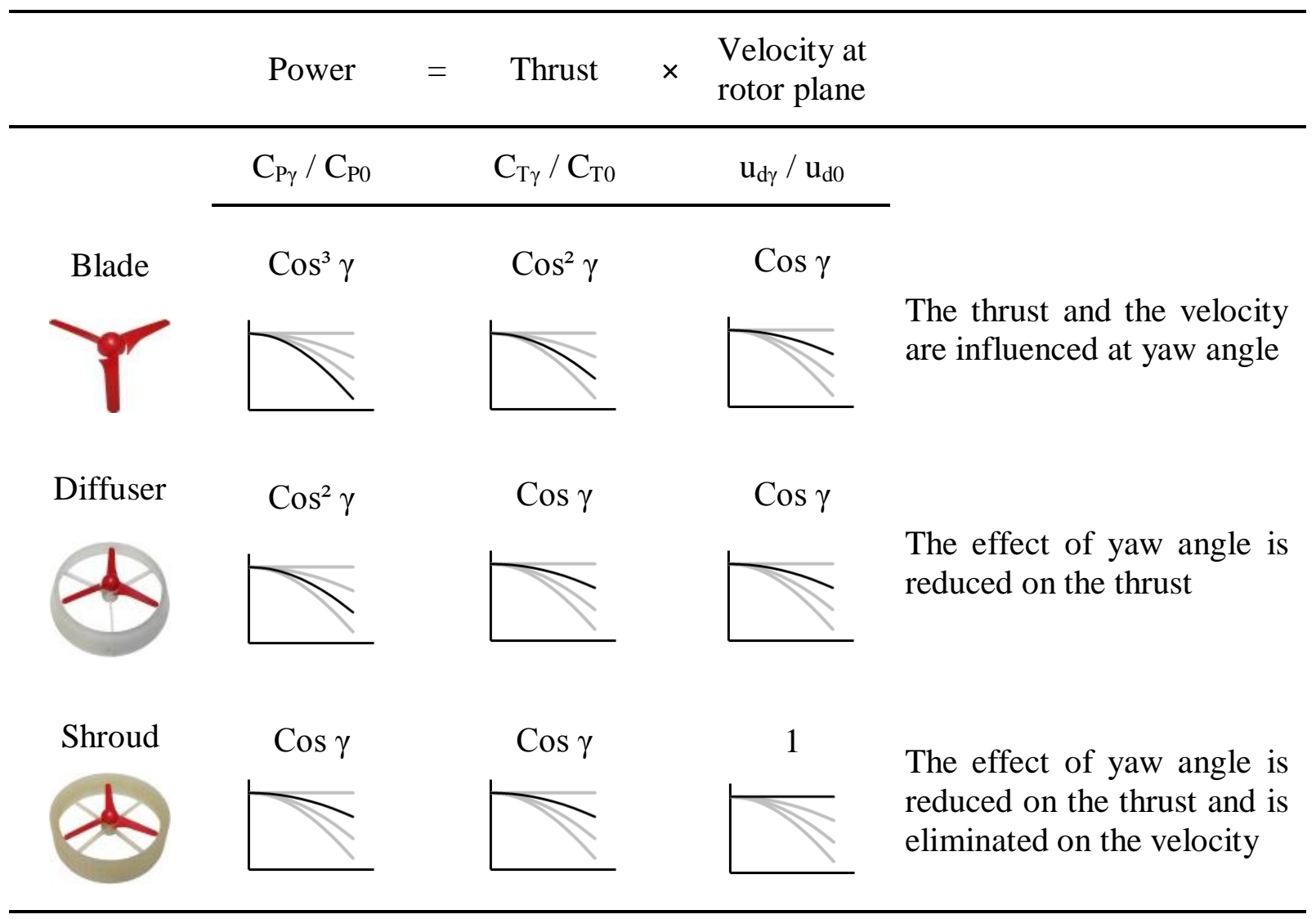

Table 6.4 is useful to help decide for a given application the role of a shroud and how it will be used to enhance the economics of a marine turbine. 


\section{Chapter 7 Conclusions and Recommendations}

\subsection{Conclusions}

A $19.8 \mathrm{~cm}$ diameter horizontal axis hydrokinetic turbine and two shroud designs are optimized, fabricated and experimentally tested in a water tunnel. The experiments aimed to study the effect of shroud on the performance of a hydrokinetic turbine in inline and off-axis flow conditions. Consistent power, torque, and thrust coefficient curves for the shrouded and unshrouded turbines are obtained for the entire range of performance curve. Free surface proximity and blockage effects are corrected using a one-dimensional actuator disk model. The conclusions from the inline operation experiments are summarized in the next section. Section 7.1.2 includes the conclusions obtained from the experiments in off-axis turbine operations.

\subsubsection{Inline Operations}

A maximum power enhancement of $91 \%$ over the unshrouded turbine is obtained with the straight wall diffuser design. The maximum power coefficients are seen to increase with the Reynolds number but at a decreasing rate. The Reynolds dependent behaviour of the power coefficients decreases at the higher flow velocities and disappears eventually. It is observed that a shroud is more effective in the low speed water currents. The power 
coefficients of the model turbine with the diffuser calculated based on the diffuser exit area is found comparable to the performance coefficients of the unshrouded turbine with the size of the diffuser exit diameter. This is in agreement with the van Bussel momentum theory which states the achievable power for a diffuser augmented wind turbine is comparable with the power of a bare turbine with the size of the diffuser exit diameter. It is also found that at the water velocities exceeding $1 \mathrm{~m} / \mathrm{s}$, an extended blade having a diameter equal to the exit diameter of the diffuser generates the same power as the model turbine with the diffuser. An extended blade can substitute for a smaller blade and a diffuser to generate the same power. This could be considered as a superior choice for the industry. However, a smaller shrouded blade can be a better choice for low speed flow sites, shallow water currents, and where marine life is of concern. Unlike wind applications, decision to use a shroud for hydrokinetic power generation could be justifiable based on the system optimization, and not by the apparent increase in the power coefficients. As the recommended conditions for a reliable comparison of a model turbine and a full scale turbine are met in all of our experiments, the results are assumed to remain valid for the higher Reynolds numbers of the full scale experiments.

\subsubsection{Yaw Operations}

The output power of a hydrokinetic turbine decreases in off-axis operations compared to inline operations. The reduction is negligible up to a $10^{\circ}$ yaw angle but increases as the yaw angle increases beyond $10^{\circ}$. In addition to reducing the power, off-axis operation also shortens the operating range of a turbine. The model turbine with the shroud, the design with the inlet, experiences negligible performance loss in yaw operations 
compared to the unshrouded turbine. The maximum reduction in the performance of the model turbine with the shroud is $6 \%$ in average at the three Reynolds numbers examined which is $17 \%$ less than the power reduction of the unshrouded turbine. Additionally, the shroud keeps the operating range of the turbine unchanged. The cosine rules of the linear momentum theory for reduction in the power and thrust of a yawed unshrouded turbine are compared to the results of several experimental studies and a close agreement is found. In an analogy to this and based on our experimental results, similar cosine relations for a shrouded turbine are proposed. The maximum power of the model turbine with the shroud and the thrust at the maximum power are experimentally observed to decrease with cosine of the yaw angle. For the model turbine with the diffuser, the maximum power and the thrust at the maximum power are observed to decrease with cosine squared and cosine of the yaw angle, respectively. Based on the cosine rules found in this study, the turbine with the shroud in a $45^{\circ}$ yawed flow would generate $29 \%$ less power than in an inline flow. The turbine with the diffuser would produce $50 \%$ less power and the unshrouded turbine, based on the cosine cubed rule of the momentum theory, would generate $65 \%$ less power in the same flow. The well-designed inlet of the shroud is capable of compensating the effect of yawed inflow which results in the better performance of the shroud over the diffuser. This characteristic of the shroud is expected to be a function of the yaw angle and is anticipated to alter in higher angles as the inlet angle of the shroud is close to the maximum yaw angle investigated. 


\subsection{Recommendations}

The author combined two methods available in the literature to correct the experimental results for the free surface proximity and blockage effects. Two previous methods have been verified to give valid corrections however the combination is proposed and used for the first time here. It is beneficial to experimentally examine the precision of such a correction compared to the previous methods. This can be done by comparing the results of tunnel tests and field experiments. Particle Image Velocimetry (PIV) of the flow over the shrouded turbines would provide a better understanding of the physics of the flow explained in this thesis. Any possible flow separation on the blades or on the shrouds' wall over the entire range of the performance curve would be captured in a PIV test. Wake study of shrouded and unshrouded turbines provides valuable information on the spacing of turbines in planning a hydrokinetic turbine farm. Combination of shrouded and unshrouded turbines can also be examined to find an optimum arrangement for a turbine farm. An analytical study is recommended to see if the cosine rules obtained in the experiments for yaw operations of a shrouded turbine can be derived. The maximum yaw angle examined in the experiments, $25^{\circ}$, is close to the inlet convergence angle of the shroud, $27^{\circ}$. It is of interest to conduct experiments in order to investigate the performance of the shroud at larger yaw angles. In such experiments the validity of the obtained cosine rules for shrouded turbines would also be examined at the higher yaw angles. 


\section{References}

Abe, K., Kihara, H., Sakurai, A., Wada, E., Sato, K., Nishida, M., Ohya, Y., 2006. An experimental study of tip-vortex structures behind a small wind turbine with a flanged diffuser. Wind Struct. 9, 413-417.

Abe, K., Nishida, M., Sakurai, A., Ohya, Y., Kihara, H., Wada, E., Sato, K., 2005. Experimental and numerical investigations of flow fields behind a small wind turbine with a flanged diffuser. J. Wind Eng. Ind. Aerodyn. 93, 951-970.

Abe, K., Ohya, Y., 2004. An investigation of flow fields around flanged diffusers using CFD. J. Wind Eng. Ind. Aerodyn. 92, 315-330.

Adaramola, M.S., Krogstad, P- $\AA .$, 2011. Experimental investigation of wake effects on wind turbine performance. Renew. Energy 36, 2078-2086.

Ahmed, M.R., 2012. Blade sections for wind turbine and tidal current turbine applications - current status and future challenges. Int. J. Energy Res. 36, 829-844.

Alfredsson, P-H., Dahlberg, J-Å., 1979. A Preliminary Wind Tunnel Study of Windmill Wake Dispersion in Various Flow Conditions. Technical Note AU-1499, Part 7, FFA. Stockholm, Sweden.

Amaral, S., Perkins, N., Giza, D., McMahon, B., 2011. Evaluation of Fish Injury and Mortality Associated with Hydrokinetic Turbines. Report 1024569, Electric Power Research Institute. Palo Alto, USA.

Anderson, M.B., Milborrow, D.J., Ross, J.N., 1982. Performance and wake measurements on a $3 \mathrm{~m}$ diameter horizontal axis wind turbine. Comparison of theory, wind tunnel and field test data. In: Fourth International Symposium on Wind Energy Systems, Stockholm, Sweden, pp. 113-135.

ANSI/API Standard 661, 2006. Air-Cooled Heat Exchangers for General Refinery Service, sixth ed. American Petroleum Institute.

Atligan, M., 1976. Swirling Flow Through Annular Diffusers with Solid Body Rotation. University of Newcastle upon Tyne.

Azad, R.S., 1996. Turbulent flow in a conical diffuser: A review. Exp. Therm. Fluid Sci. $13,318-337$.

Bahaj, A.S., 2011. Generating electricity from the oceans. Renew. Sustain. Energy Rev. $15,3399-3416$. 
Bahaj, A.S., Batten, W.M.J., McCann, G., 2007a. Experimental verifications of numerical predictions for the hydrodynamic performance of horizontal axis marine current turbines. Renew. Energy 32, 2479-2490.

Bahaj, A.S., Molland, A.F., Chaplin, J.R., Batten, W.M.J., 2007b. Power and thrust measurements of marine current turbines under various hydrodynamic flow conditions in a cavitation tunnel and a towing tank. Renew. Energy 32, 407-426.

Barber, S., Wang, Y., Jafari, S., Chokani, N., Abhari, R.S., 2011. The impact of ice formation on wind turbine performance and aerodynamics. J. Sol. Energy Eng. 133, 011007.

Barlow, J.B., Rae, W.H., Pope, A., 1999. Low-Speed Wind Tunnel Testing. third ed. Wiley, New York.

Barnsley, M.J., Wellicome, J.F., 1990. Final Report on the 2nd Phase of Development and Testing of a Horizontal Axis Wind Turbine Test Rig for the Investigation of Stall Regulation Aerodynamics. Carried out under ETSU Agreement E.5A/ CON5103/1746.

Batten, W.M.J., Bahaj, A.S., Molland, A.F., Chaplin, J.R., 2006. Hydrodynamics of marine current turbines. Renew. Energy 31, 249-256.

Batten, W.M.J., Bahaj, A.S., Molland, A.F., Chaplin, J.R., 2007. Experimentally validated numerical method for the hydrodynamic design of horizontal axis tidal turbines. Ocean Eng. 34, 1013-1020.

Batten, W.M.J., Bahaj, A.S., Molland, A.F., Chaplin, J.R., 2008. The prediction of the hydrodynamic performance of marine current turbines. Renew. Energy 33, 10851096.

Bedard, R., 2008. Prioritized Research Deployment and Demonstration (RDD \& D) Needs: Marine and Other Hydrokinetic Renewable Energy. Electric Power Research Institute. Palo Alto, USA.

Bell, S., 1999. A Beginner's Guide to Uncertainty of Measurement. Measurement Good Practice Guide No. 11 (Issue 2). National Physical Laboratory. Teddington, UK.

Bet, F., Grassmann, H., 2003. Upgrading conventional wind turbines. Renew. Energy 28, $71-78$.

Betz, A., 1920. Das maximum der theoretisch möglichen ausnutzüng des windes durch windmotoren. Zeitschrift fur das Gesamte Turbinenwesen. 17, 307-309.

Betz, A., 1926. Wind-Energie und ihre Ausnutzung durch Windmühlen. Vandenhoeck and Ruprecht, Göttingen. 
Birjandi, A.H., Bibeau, E.L., Chatoorgoon, V., Kumar, A., 2013. Power measurement of hydrokinetic turbines with free-surface and blockage effect. Ocean Eng. 69, 9-17.

Birjandi, A.H., d'Auteuil, S., Ridd, C., Bibeau, E.L., 2015. An innovative low cost hydrokinetic site selection technique for cold climate regions, In: OCEANS ' 15 MTS/IEEE Genova Conference, Genova, Italy.

Boorsma, K., 2012. Power and Loads for Wind Turbines in Yawed Conditions. Technical Report ECN-E--12-047, ECN. The Netherlands.

Burton, T., Sharpe, D., Jenkins, N., Bossanyi, E., 2001. Wind Energy Handbook. Wiley, New York.

Busch, D.S., Greene, C.M., Good, T.P., 2013. Estimating effects of tidal power projects and climate change on threatened and endangered marine species and their food web. Conserv. Biol. 27, 1190-1200.

Cabuk, H., Modi, V., 1992. Optimum plane diffusers in laminar flow. J. Fluid Mech. 237, 373-393.

Cada, G., Ahlgrimm, J., Bahleda, M., Bigford, T., Stavrakas, S.D., Hall, D., Moursund, R., Sale, M., 2007. Potential impacts of hydrokinetic and wave energy conversion technologies on aquatic environments. Fisheries 32, 174-181.

Chang, P.K., 1976. Control of Flow Separation: Energy Conservation, Operational Efficiency, and Safety, first ed. Hemisphere Publishing Corporation, Washington.

Chatfield, C., 1983. Statistics for Technology, third ed. Chapman and Hall, New York.

Chen, T.Y., Liou, L.R., 2011. Blockage corrections in wind tunnel tests of small horizontal-axis wind turbines. Exp. Therm. Fluid Sci. 35, 565-569.

Choi, K.S., DeBisschop, J.R., Clayton, B.R., 1998. Turbulent boundary-layer control by means of spanwise-wall oscillation. AIAA J. 36, 1157-1163.

Clausen, P., 1988. Measurements and Predictions of Swirling Flow Through an Axisymmetric Diffuser. The University of New Castle.

Clayton, B.R., Filby, P., 1982. Measured effects of oblique flows and change in blade pitch angle on performance and wake development of model wind turbines. In: 4th BWEA Wind Energy Conference. Cranfield Institute of Technology.

Coiro, D.P., Troise, G., Scherillo, F., 2012. Design, towing tank test and deployment of full scale GEM, a novel tethered system for harnessing tidal energy. In: Asian Wave and Tidal Conference Series, Jeju Island, Korea. 
Corten, G.P., 2001. Flow Separation on Wind Turbine Blades. University of Utrecht.

Coton, F.N., Wang, T., 1999. The prediction of horizontal axis wind turbine performance in yawed flow using an unsteady prescribed wake model. Proc. Inst. Mech. Eng. Part A J. Power Energy 213, 33-43.

de Robertis, A., Wilson, C.D., 2006. Walleye pollock respond to trawling vessels. ICES J. Mar. Sci. 63, 514-522.

de Vries, O., 1979. Fluid Dynamic Aspects of Wind Energy Conversion. AGARDograph No. 243, AGARD-AG-243.

de Vries, O., 1983. On the theory of the horizontal-axis wind turbine. Annu. Rev. Fluid Mech. 15, 77-96.

de Vries, O., 1985. Comment on the yaw stability of a horizontal-axis wind turbine at small angles of attack. Wind Eng. 9, 42-49.

Dick, E., 1986. Power limits for wind energy concentrator systems. Wind Eng. 10, 98115.

Dietrich, C.F., 1991. Uncertainty, Calibration and Probability, second ed. Taylor \& Francis Group, USA.

Drouen, L., Charpentier, J.F., Semail, E., Clenet, S., 2007. Study of an innovative electrical machine fitted to marine current turbines. In: OCEANS 2007 Europe Conference, pp. 1-6.

Ebert, P.R., Wood, D.H., 1997. The near wake of a model horizontal-axis wind turbineI. Experimental arrangements and initial results. Renew. Energy 12, 225-243.

Ebert, P.R., Wood, D.H., 2001. The near wake of a model horizontal-axis wind turbine Part 3: properties of the tip and hub vortices. Renew. Energy 22, 461-472.

Eggleston, D.M., Stoddard, F.S., 1987. Wind Turbine Engineering Design. Van Nostrand Reinhold, New York.

Eldridge, F.R., 1975. Wind Machines. Report NSF-RA-N-75-051, National Science Foundation. Washington, USA.

ElQatary, I., Elhadidi, B., 2014. Comparison between OpenFOAM CFD \& BEM theory for variable speed - variable pitch HAWT. ITM Web Conf. 2, 05001.

Eriksson, S., Bernhoff, H., Leijon, M., 2008. Evaluation of different turbine concepts for wind power. Renew. Sustain. Energy Rev. 12, 1419-1434. 
Fichaux, N., Beurskens, J., Jensen, P.H., Wilkes, J., Frandsen, S., Sørensen, J.D., Eecen, P., Malamatenios, C., Gomez, J.A., Hemmelmann, J., van Kuik, G., Bulder, B., Rasmussen, F., Janssen, B., Fischer, T., Bossanyi, E., Courtney, M., Giebhardt, J., Barthelmie, R., Holmstrøm, O., 2011. UpWind, Design Limits and Solutions for Very Large Wind Turbines. Contract Number 019945 (SES6), Ris $\varnothing$ National Laboratory - DTU. Denmark.

Fletcher, C.A.J., 1981. Computational analysis of diffuser-augmented wind turbines. Energy Convers. Manag. 21, 175-183.

Foreman, K.M., Gilbert, B., Oman, R.A., 1978. Diffuser augmentation of wind turbines. Soalr Energy 20, 305-311.

Foreman, K.M., Gilbert, B.L., 1979a. Technical development of the diffuser augmented wind turbine (DAWT) concept. Wind Eng. 3, 153-166.

Foreman, K.M., Gilbert, B.L., 1979b. Further Investigations of Diffuser Augmented Wind Turbines, Parts I \& II. Report RE-585, Final Report on DOE Contract No. EY-76-C-02-2616.M002, Grumman Research Dept. New York.

Foreman, K.M., Gilbert, B.L., 1983. A Free Jet Wind Tunnel Investigation of DAWT Models. Report RE-668, SERI/TR 01311-1, Grumman Research Dept. New York.

Foreman, K.M., Maciulaitis, A., Gilbert, B.L., 1983. Performance predictions and recent data for advanced DAWT models. Submitted for the April 1983 ASME Solar Energy Division Meeting, Grumman Research Dept. New York.

Fox, R.W., McDonald, A.T., Pritchard, P.J., 2004. Introduction to Fluid Mechanics, sixth ed. Wiley, USA.

Fraenkel, P., 2010. Practical tidal turbine design considerations: a review of technical alternatives and key design decisions leading to the development of the SeaGen 1.2 MW tidal turbine. In: Fluid Machinery Group - Ocean Power Fluid Machinery Seminar. Institution of Mechanical Engineers, London, pp. 1-19.

Fraenkel, P.L., 2002. Power from marine currents. Proc. Inst. Mech. Eng. Part A J. Power Energy 216, 1-14.

Fraenkel, P.L., 2006. Tidal current energy technologies. Ibis 148, 145-151.

Frankovic, B., Vrsalovic, I., 2001. New high profitable wind turbines. Renew. Energy 24, 491-499.

Gad-el-Hak, M., Bushnell, D.M., 1991. Separation control: Review. J. Fluids Eng. 113, 5-30. 
Gaden, D.L.F., 2007. An Investigation of River Kinetic Turbines: Performance Enhancements, Turbine Modelling Techniques, and an Assessment of Turbulence Models. University of Manitoba.

Gaden, D.L.F., Bibeau, E.L., 2010. A numerical investigation into the effect of diffusers on the performance of hydro kinetic turbines using a validated momentum source turbine model. Renew. Energy 35, 1152-1158.

Gaonkar, G.H., Peters, D.A., 1986. Effectiveness of current dynamic-inflow models in hover and forward flight. J. Am. Helicopter Soc. 31, 47-57.

Garrett, C., Cummins, P., 2007. The efficiency of a turbine in a tidal channel. J. Fluid Mech. 588, 243-251.

Gersten, K., 1966. The Separation of the Turbulent Boundary Layer as Influenced by Blowing, Suction, Moving Wall, and Propeller Slipstream in "Separated Flows." AGARD-CP-4, Belgium.

Ghose, S., Kline, S.J., 1978. The computation of optimum pressure recovery in twodimensional diffusers. J. Fluids Eng. 100, 419-426.

Gilbert, B.L., Foreman, K.M., 1979. Experimental demonstration of the diffuseraugmented wind turbine concept. Jounal of Energy 3, 235-240.

Gilbert, B.L., Foreman, K.M., 1983. Experiments with a diffuser-augmented model wind turbine. Trans. ASME J. Energy Resour. Technol. 105, 46-53.

Gilbert, B.L., Oman, R.A., Foreman, K.M., 1978. Fluid dynamics of diffuser-augmented wind turbines. Jounal of Energy 2, 368-374.

Glauert, H., 1947. The Elements of Aerofoil and Airscrew Theory, second ed. Cambridge University Press, New York.

Grant, I., Parkin, P., Wang, X., 1997. Optical vortex tracking studies of a horizontal axis wind turbine in yaw using laser-sheet, flow visualisation. Exp. Fluids 23, 513-519.

Grassmann, H., Bet, F., Cabras, G., Ceschia, M., Cobai, D., DelPapa, C., 2003a. A partially static turbine-first experimental results. Renew. Energy 28, 1779-1785.

Grassmann, H., Bet, F., Ceschia, M., Ganis, M.L., 2003b. On the physics of partially static turbines. Renew. Energy 29, 491-499.

Güney, M.S., Kaygusuz, K., 2010. Hydrokinetic energy conversion systems: A technology status review. Renew. Sustain. Energy Rev. 14, 2996-3004. 
Haans, W., 2011. Wind Turbine Aerodynamics in Yaw Unravelling the Measured Rotor Wake. Delft University of Technology.

Haans, W., Sant, T., van Kuik, G., van Bussel, G., 2005. Measurement of tip vortex paths in the wake of a HAWT under yawed flow conditions. J. Sol. Energy Eng. 127, 456-463.

Hand, M.M., Simms, D.A., Fingersh, L.J., Jager, D.W., Cotrell, J.R., Schreck, S., Larwood, S.M., 2001. Unsteady Aerodynamics Experiment Phase VI: Wind Tunnel Test Configurations and Available Data Campaigns. Report NREL/TP-500-29955, NREL. Golden, USA.

Hansen, A.C., 1992. Yaw Dynamics of Horizontal Axis Wind Turbines. Report NREL/TP-442-4822, University of Utah. Salt Lake City, USA.

Hansen, A.C., Butterfield, C.P., 1993. Aerodynamics of horizontal-axis wind turbines. Annu. Rev. Fluid Mech. 25, 115-149.

Hansen, A.C., Butterfield, C.P., Cui, X., 1990. Yaw loads and motions of a horizontal axis wind turbine. J. Sol. Energy Eng. 112, 310-314.

Hansen, A.C., Cui, X., 1989. Analyses and observations of wind turbine yaw dynamics. J. Sol. Energy Eng. 111, 367-371.

Hansen, M.O.L., Sørensen, N.N., Flay, R.G.J., 2000. Effect of placing a diffuser around a wind turbine. Wind Energy 3, 207-213.

Hasegawa, Y., Kkikuyama, K., Tanimoto, M., van Bussel, G.J.W., 1996. Calculation of yawed inflow effects on a horizontal axis wind turbine rotor by an asymptotic acceleration potential method. In: European Union Wind Energy Conference, Göteborg, Sweden, pp. 753-756.

Hassan, H.F., Karim, O.A., El-Shafie, A., 2012. Tidal current turbines glance at the past and look into future prospects in Malaysia. Renew. Sustain. Energy Rev. 16, 57075717.

Hau, E., von Renouard, H., 2013. Wind Turbines: Fundamentals, Technologies, Application, Economics, third trans. ed. Springer.

Hussain, A.K.M.F., Ramjee, V., 1976. Effects of the axisymmetric contraction shape on incompressible turbulent flow. J. Fluids Eng. 98, 58-69.

Igra, O., 1976. Shrouds for aerogenerators. AIAA J. 14, 1481-1483.

Igra, O., 1977. The shrouded aerogenerator. Jounal of Energy 2, 429-439. 
Igra, O., 1981. Research and development for shrouded wind turbines. Energy Convers. Manag. 21, 13-48.

Igra, O., 1984. Research and developement for shrouded wind turbines. In: European Wind Energy Conference 1984, Hamburg, Germany.

Isensee, G.M., Abdul-Razzak, H., 2012. Modeling and analysis of diffuser augmented wind turbine. Int. J. Energy Sci. 2, 84-88.

Iwasaki, M., 1953. The experimental and theoretical investigations of windmills. Reports Res. Inst. Appl. Mech. 2, 181-228.

Jamieson, P., 2011. Innovation in Wind Turbine Design, first ed. Wiley, UK.

JCGM, 2008. Evaluation of Measurement Data - Guide to the Expression of Uncertainty in Measurement, JCGM 100:2008 (GUM 1995 with minor corrections). 〈http://www.bipm.org/utils/common/documents/jcgm/JCGM_100_2008_E.pdf〉.

Ketchman, J.W., 2010. Design, Build and Test of an Axial Flow Hydrokinetic Turbine with Fatigue Analysis. Massachusetts Institute of Technology.

Khan, M.J., Bhuyan, G., Iqbal, M.T., Quaicoe, J.E., 2009. Hydrokinetic energy conversion systems and assessment of horizontal and vertical axis turbines for river and tidal applications: A technology status review. Appl. Energy 86, 1823-1835.

Khan, M.J., Iqbal, M.T., Quaicoe, J.E., 2008. River current energy conversion systems: Progress, prospects and challenges. Renew. Sustain. Energy Rev. 12, 2177-2193.

Kirke, B.K., 2011. Tests on ducted and bare helical and straight blade Darrieus hydrokinetic turbines. Renew. Energy 36, 3013-3022.

Klein, A., 1981. Review: Effects of inlet conditions on conical-diffuser performance. J. Fluids Eng. 103, 250-257.

Kline, S.J., Abbott, D.E., Fox, R.W., 1959. Optimum design of straight-walled diffusers. J. Basic Eng. 81, 321-331.

Kogan, A., Seginer, A., 1963. Final Report on Shroud Design. Report No. 32A, Department of Aeronautical Engineering Technion.

Krogstad, P- $\AA$., Adaramola, M.S., 2012. Performance and near wake measurements of a model horizontal axis wind turbine. Wind Energy 15, 743-756.

Kumar, D.S., Kumar, K.L., 1980. Effect of swirl on pressure recovery in annular diffusers. J. Mech. Eng. Sci. 22, 305-313. 
Lago, L.I., Ponta, F.L., Chen, L., 2010. Advances and trends in hydrokinetic turbine systems. Energy Sustain. Dev. 14, 287-296.

Lai, Y.G., So, R.M.C., Hwang, B.C., 1989. Calculation of planar and conical diffuser flows. AIAA J. 27, 542-548.

Lawn, C.J., 2003. Optimization of the power output from ducted turbines. Proc. Inst. Mech. Eng. Part A J. Power Energy 217, 107-117.

Leishman, J.G., 2002. Challenges in modelling the unsteady aerodynamics of wind turbines. Wind Energy 5, 85-132.

Leishman, J.G., Beddoes, T.S., 1989. A semi-empirical model for dynamic stall. J. Am. Helicopter Soc. 34 (3), 3-17.

Lewis, R.I., 1991. Vortex Element Methods for Fluid Dynamic Analysis of Engineering Systems. Cambridge University Press, New York.

Lewis, R.I., Williams, J.E., Abdelghaffar, M.A., 1977. A theory and experimental investigation of ducted wind turbines. Wind Eng. 1, 104-125.

Lilley, G.M., Rainbird, W.J., 1956. A Preliminary Report on the Design and Performance of a Ducted Windmill. Report 102, College of Aeronautics. Cranfield, UK.

Loeffler Jr., A.L., 1981. Flow field analysis and performance of wind turbines employing slotted diffusers. J. Sol. Energy Eng. 103, 17-22.

Loeffler Jr., A.L., Vanderbilt, D., 1978. Inviscid flow through wide-angle diffuser with actuator disk. AIAA J. 16, 1111-1112.

Lokocz, T.A., 2012. Testing of a Ducted Axial Flow Tidal Turbine. University of Maine.

Lynette, R., 1988. California Wind Farms: Operational Data Collection And Analysis. Solar Energy Research Institute. Golden, USA.

Madsen, J.I., Shyy, W., Haftka, R.T., 2000. Response surface techniques for diffuser shape optimization. AIAA J. 38, 1512-1518.

Maeda, T., Kamada, Y., Suzuki, J., Fujioka, H., 2008. Rotor blade sectional performance under yawed inflow conditions. J. Sol. Energy Eng. 130, 031018.

Manwell, J.F., McGowan, J.G., Rogers, A.L., 2009. Wind Energy Explained: Theory, Design and Application. second ed. Wiley, UK.

Massouh, F., Dobrev, I., 2007. Exploration of the vortex wake behind of wind turbine rotor. J. Phys. Conf. Ser. 75, 012036. 
McDonald, A.T., Fox, R.W., 1966. An experimental investigation of incompressible flow in conical diffuser. Int. J. Mech. Sci. 8, 125-139.

McDonald, A.T., Fox, R.W., van Dewoestine, R.V., 1971. Effects of swirling inlet flow on pressure recovery in conical diffusers. AIAA J. 9, 2014-2018.

McKay, P., Carriveau, R., Ting, D.S-K., 2013. Wake impacts on downstream wind turbine performance and yaw alignment. Wind Energy 16, 221-234.

McTavish, S., Feszty, D., Nitzsche, F., 2012. An experimental assessment of blockage and Reynolds number effects on wind turbine wake development. In: Proceedings of ASME Turbo Expo 2012, Copenhagen, Denmark, pp. 1-10.

Mertens, S., 2006. Wind Energy in the Built Environment: Concentrator Effects of Buildings. Delft Technical University.

Mikhail, M.N., 1979. Optimum design of wind tunnel contractions. AIAA J. 17, 471477.

Morel, T., 1977. Design of two-dimensional wind tunnel contractions. J. Fluids Eng. 99, $371-378$.

Myers, L., Bahaj, A.S., 2006. Power output performance characteristics of a horizontal axis marine current turbine. Renew. Energy 31, 197-208.

Nagai, M., Irabu, K., 1987. Momentum theory of diffuser augmented wind turbine. Trans. Japan Soc. Mech. Eng. Ser. B 53, 1543-1547.

Nicholls-Lee, R.F., Turnock, S.R., 2008. Tidal energy extraction: renewable, sustainable and predictable. Sci. Prog. 91, 81-111.

Noura, B., Dobrev, I., Dizene, R., Massouh, F., Khelladi, S., 2012. Experimental study of yawed inflow around wind turbine rotor. Proc. Inst. Mech. Eng. Part A J. Power Energy 226, 664-673.

O Rourke, F., Boyle, F., Reynolds, A., 2010. Tidal energy update 2009. Appl. Energy 87, 398-409.

Ohya, Y., Karasudani, T., 2010. A shrouded wind turbine generating high output power with wind-lens technology. Energies 3, 634-649.

Ohya, Y., Karasudani, T., Sakurai, A., Abe, K., Inoue, M., 2008. Development of a shrouded wind turbine with a flanged diffuser. J. Wind Eng. Ind. Aerodyn. 96, 524539. 
Oman, R.A., Foreman, K.M., Gilbert, B.L., 1977. Investigation of Diffuser-Augmented Wind Turbines, Parts I \& II. Report RE-534, ERDA Report C00-2616-2, Grumman Research Dept. New York, USA.

Pedersen, T.F., 2004. On wind turbine power performance measurements at inclined airflow. Wind Energy 7, 163-176.

Phillips, D.G., 2003. An Investigation on Diffuser Augmented Wind Turbine Design. The University of Auckland.

Phillips, D.G., Nash, T.A., Oakey, A., Flay, R.G.J., Richards, P.J., 1999. Computational fluid dynamic and wind tunnel modelling of a diffuser augmented wind turbine. Wind Eng. 23, 7-13.

Ponta, F.L., Jacovkis, P.M., 2008. Marine-current power generation by diffuseraugmented floating hydro-turbines. Renew. Energy 33, 665-673.

Riegler, G., 1983. Principles of energy extraction from a free stream by means of wind turbines. Wind Eng. 7, 115-126.

Robertson, J., 1949. Water Tunnel Diffuser Flow Studies. ORL Report No. 7958-143, Penn State College.

Robinson, D., Coton, F., Galbraith, R.M., Vezza, M., 1995. The development of a prescribed wake model for performance prediction in steady yawed flow. In: 14th ASME/ETCE Wind Energy Symposium, Houston, USA.

Ross, I., 2010. Wind Tunnel Blockage Corrections: An Application to Vertical-Axis Wind Turbines. University of Dayton.

Ross, I., Altman, A., 2011. Wind tunnel blockage corrections: Review and application to Savonius vertical-axis wind turbines. J. Wind Eng. Ind. Aerodyn. 99, 523-538.

Sanuki, M., 1950a. Studies on biplane wind vanes, ventilator tubes and cup anemometers (II). Pap. Meteorol. Geophys. 1, 227-298.

Sanuki, M., 1950b. Studies on biplane wind vanes, ventilator tubes and cup anemometers (I). Pap. Meteorol. Geophys. 1, 81-132.

Savkar, S.D., 1980. Derivation of Kline line a-a for straight walled diffusers from stratford's separation criterion. J. Fluids Eng. 102, 497-498.

Shepherd, D.G., 1990. Historical Development of the Windmill. NASA Contractor Report 4337, DOE/NASA/5266-1, Cornell University. Ithaca, USA. 
Scherillo, F., Maisto, U., Troise, G., Coiro, D.P., Miranda, S., 2011. Numerical and experimental analysis of a shrouded hydroturbine. In: International Conference on Clean Electrical Power (ICCEP). pp. 216-222.

Schreck, S.J., Robinson, M.C., Hand, M.M., Simms, D.A., 2001. Blade dynamic stall vortex kinematics for a horizontal axis wind turbine in yawed conditions. J. Sol. Energy Eng. 123, 272-281.

Schumack, M., 1979. Results of Wind Tunnel Tests on the Scale Model of the THE I/2 Rotor. Report R408S, Wind Energy Group, Department of Physics, Eindhoven University of Technology.

Schweizer, P.E., Cada, G.F., Bevelhimer, M.S., 2012. Laboratory Experiments on the Effects of Blade Strike from Hydrokinetic Energy Technologies on Larval and Juvenile Freshwater Fishes. Report ORNL/TM-2012/108, Oak Ridge National Laboratory. Oak Ridge, USA.

Setoguchi, T., Shiomi, N., Kaneko, K., 2004. Development of two-way diffuser for fluid energy conversion system. Renew. Energy 29, 1757-1771.

Shahsavarifard, M., Bibeau, E.L., 2013. Effect of shroud on the performance of horizontal axis hydrokinetic turbines. In: 4th Annual Marine Renewable Energy Technical Conference. Providence, USA.

Shahsavarifard, M., Bibeau, E.L., 2014a. Experimental Power and Thrust Coefficients of a Shrouded Horizontal Axis Hydrokinetic Turbine. doi: 10.5203/ds_sha_1.

Shahsavarifard, M., Bibeau, E.L., 2014b. Experimental Power and Thrust Coefficients of a Shrouded Horizontal Axis Hydrokinetic Turbine in Yaw Operation. doi: 10.5203/ds_sha_2.

Shahsavarifard, M., Bibeau, E.L., Birjandi, A.H., 2013. Performance gain of a horizontal axis hydrokinetic turbine using shroud. In: OCEANS '13 MTS/IEEE San Diego Conference, San Diego, USA.

Shahsavarifard, M., Bibeau, E.L., Chatoorgoon, V., 2015. Effect of shroud on the performance of horizontal axis hydrokinetic turbines. Ocean Eng. 96, 215-225.

Shahsavarifard, M., Birjandi, A.H., Bibeau, E.L., 2014a. Measurements Using a Hydrokinetic Screw-Type Impeller Turbine. Technical Report CHTTC/TR-0010814, Canadian Hydrokinetic Turbine Test Center. Winnipeg, Canada.

Shahsavarifard, M., Birjandi, A.H., Bibeau, E.L., 2014b. Performance Test of Energy Cat 3EC42 Hydrokinetic Turbine. Technical Report CHTTC/TR-002-1014, Canadian Hydrokinetic Turbine Test Center. Winnipeg, Canada. 
Shives, M., Crawford, C., 2011. Developing an empirical model for ducted tidal turbine performance using numerical simulation results. Proc. Inst. Mech. Eng. Part A J. Power Energy 226, 112-125.

Smulders, P.T., Lenssen, G., van Leeuwen, H., 1981. Experiments with wind rotors in yaw. In: International Symposium on "Aplication of Fluid Mechanics and Heat Transfer to Energy and Environmental Problems," Patras, Greece, pp. 1-21.

Souder, H.C., Li, Y., Thresher, R., 2010. Marine hydrokinetic turbine technology and the environment: Device-biota interactions. In: OCEANS 2010 MTS/IEEE Seattle Conference, Seattle, USA.

Strawn, R.C., Kline, S.J., 1983. A stall margin design method for planar and axisymmetric diffusers. J. Fluids Eng. 105, 28-33.

Swift, A.H.P., 1981. The Effects of Yawed Flow on Wind Turbine Rotors. Washington University.

Takahashi, S., Hata, Y., Ohya, Y., Karasudani, T., Uchida, T., 2012. Behavior of the blade tip vortices of a wind turbine equipped with a brimmed-diffuser shroud. Energies 5, 5229-5242.

Taylor, J., 2011. Charting the Course: Canada's Marine Renewable Energy Technology Roadmap.

ten Hoopen, P.D.C., 2009. An Experimental and Computational Investigation of a Diffuser Augmented Wind Turbine with an Application of Vortex Generators on the Diffuser Trailing Edge. Delft University of Technology.

Tennant, J.S., 1973. A subsonic diffuser with moving walls for boundary-layer control. AIAA J. 11, 240-242.

Tong, W., 2010. Wind Power Generation and Wind Turbine Design. WIT Press, UK.

Toshimitsu, K., Nishikawa, K., Haruki, W., Oono, S., Takao, M., Ohya, Y., 2008. PIV measurements of flows around the wind turbines with a flanged-diffuser shroud. J. Therm. Sci. 17, 375-380.

Tsalicoglou, C., Barber, S., Chokani, N., Abhari, R.S., 2012. Effect of flow inclination on wind turbine performance. J. Eng. Gas Turbines Power 134, 122601.

Vabø, R., Olsen, K., Huse, I., 2002. The effect of vessel avoidance of wintering Norwegian spring spawning herring. Fish. Res. 58, 57-77.

van Bussel, G.J.W., 1994. Induced velocity distributions and axial loads on yawed rotors. In: European Wind Energy Conference, Thessaloniki, Greece, pp. 10-14. 
van Bussel, G.J.W., 1998. Development of a Momentum Theory for DAWT's (Diffuser Augmented Wind Turbines). Mie University, Tsu, Japan.

van Bussel, G.J.W., 1999. An assessment of the performance of diffuser augmented wind turbines (DAWT's). In: 3rd ASME/JSME Joint Fluids Engineering Conference, San Francisco, USA.

van Bussel, G.J.W., 2007. The science of making more torque from wind: Diffuser experiments and theory revisited. J. Phys. Conf. Ser. 75, 012010.

van Dorst, F.A., 2011. An Improved Rotor Design for a Diffuser Augmented Wind Turbine. Eindhoven University of Technology.

van Holten, Th., 1981. Concentrator systems for wind energy, with emphasis on tipvanes. Wind Eng. 5 (1), 29-45.

Vermaak, H.J., Kusakana, K., Koko, S.P., 2014. Status of micro-hydrokinetic river technology in rural applications: A review of literature. Renew. Sustain. Energy Rev. 29, 625-633.

Vermeer, L.J., 2001. A review of wind turbine wake research at TUDelft. In: A Collection of the 2001 ASME Wind Energy Symposium Technical Papers. ASME, New York, pp. 103-113.

Vermeer, L.J., Sørensen, J.N., Crespo, A., 2003. Wind turbine wake aerodynamics. Prog. Aerosp. Sci. 39, 467-510.

Viehman, H.A., Zydlewski, G.B., 2014. Fish interactions with a commercial-scale tidal energy device in the natural environment. Estuaries and Coasts 38, 1-12.

Whelan, J.I., Graham, J.M.R., Peiró, J., 2009. A free-surface and blockage correction for tidal turbines. J. Fluid Mech. 624, 281-291.

Widnall, S., 2009. Potential Flow Calculations of Axisymmetric Ducted Wind Turbines. Massachusetts Institute of Technology. Cambridge, USA.

Willmer, A.C., 1979. The aerodynamic design and testing of a vertical-axis windmill. In: International Conference on Future Energy Concepts. Institution of Electrical Engineers, pp. 286-289.

Wilson, B., Batty, R.S., Daunt, F., Carter, C., 2007. Collision Risks Between Marine Renewable Energy Devices and Mammals, Fish and Diving Birds. Report to the Scottish Executive, Scottish Association for Marine Science. Oban, Scotland.

Wilson, R.E., 1980. Wind-turbine aerodynamics. J. Wind Eng. Ind. Aerodyn. 5, 357-372. 
Wilson, R.E., 2009. Wind Turbine Technology: Fundamental Concepts in Wind Turbine Engineering, second ed. ASME Press.

Wilson, R.E., Lissaman, P.B.S., 1974. Applied Aerodynamics of Wind Power Machines. Supported by NSF, RANN Under Grant No. GI-418340, Department of Mechanical Engineering, Oregon State University. Corvallis, USA.

Wilson, R.E., Lissaman, P.B.S., Walker, S.N., 1976. Aerodynamic Performance of Wind Turbines. Technical Report ERDA/NSF/04014-76/1, Energy Research and Development Administration. Corvallis, USA.

Wortman, A.J., 1983. Introduction to Wind Turbine Engineering. Butterworth Publishers, Boston.

Yuce, M.I., Muratoglu, A., 2015. Hydrokinetic energy conversion systems: A technology status review. Renew. Sustain. Energy Rev. 43, 72-82. 


\section{Appendix}

Drawings of the test rig and some of its components are presented here. These are part of the drawings generated by the present author to build the test model at the university machine shop. The drawings changed several times for different reasons such as difficulty or impossibility of fabrication. Some parts have been designed and built several times before obtaining the optimum design. The entire test rig was redesigned and rebuilt after finding the results of the first model unreliable in multiple tests. Different components like the small gearbox and the motor were ordered a few times and a speed reducer was later added to the motor in order to best meet the experiments conditions. Thanks to our careful machinist, Irwin Penner who I benefitted from his ideas during the fabrication process, pieces were machined to the highest precision needed for an accurate experimental work. This resulted in the perfect adjustment of the components which was a great advantage during the assembly. High precision Laser bender was used to build the V-shape support in order to ensure it perfectly lines up with the other components. Although every effort was made in the design and fabrication process, it took a while to perfectly adjust the different components of the apparatus before repeatable results could be obtained in multiple tests during the experiments. The design, fabrication, error diagnosis and problem solving were the most time consuming parts of the project before any reliable results could be obtained and analysed. 


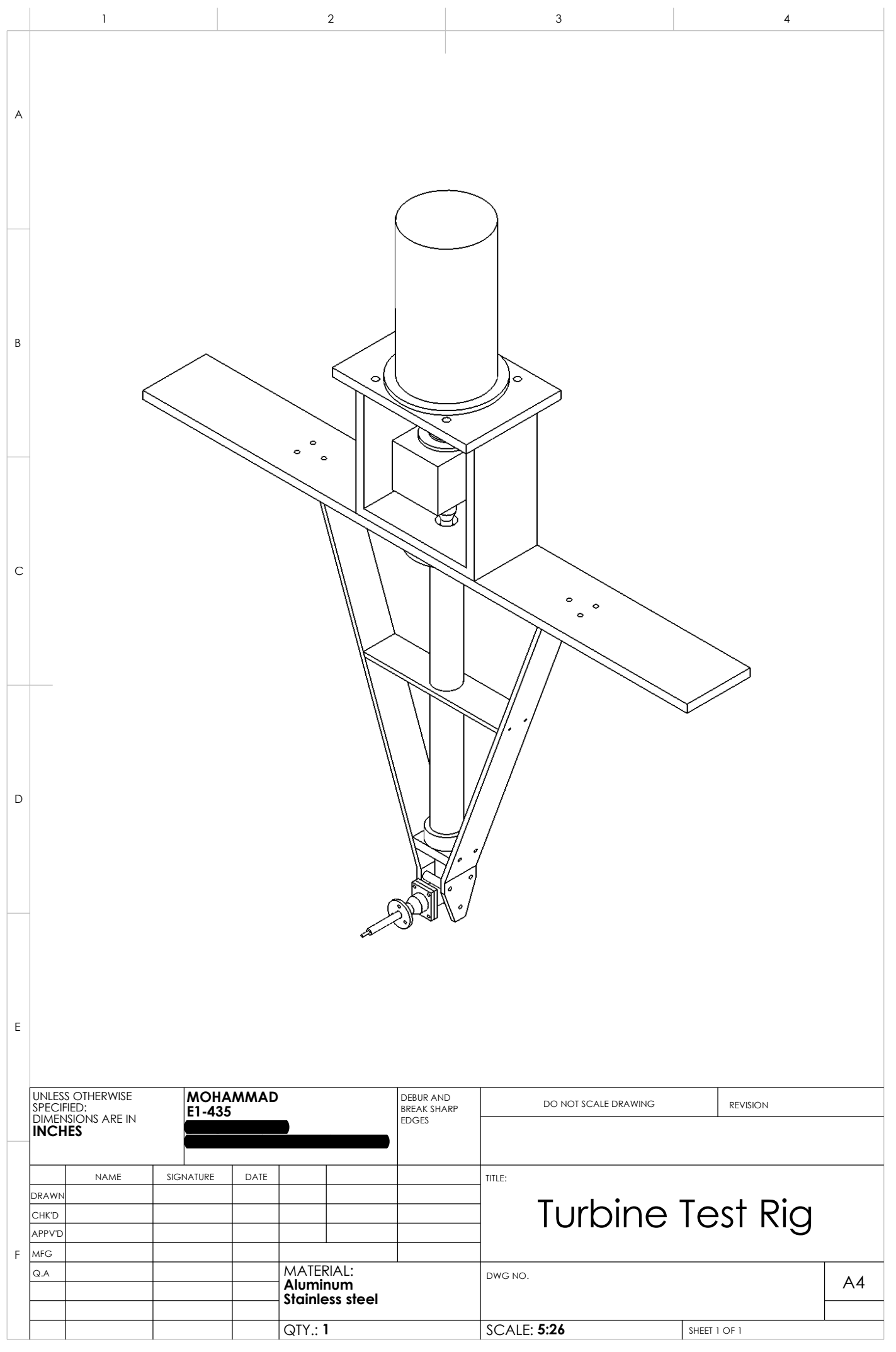




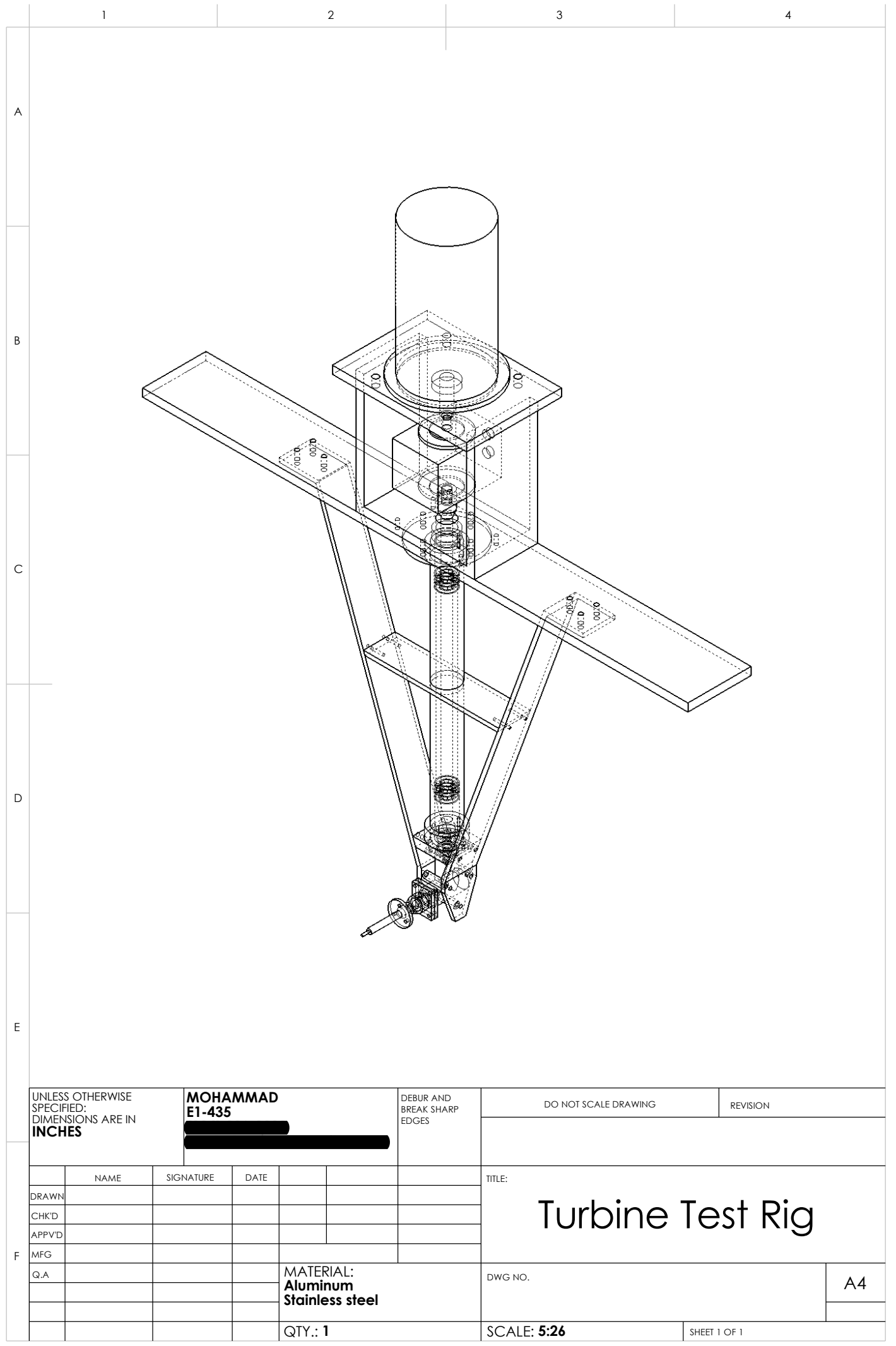




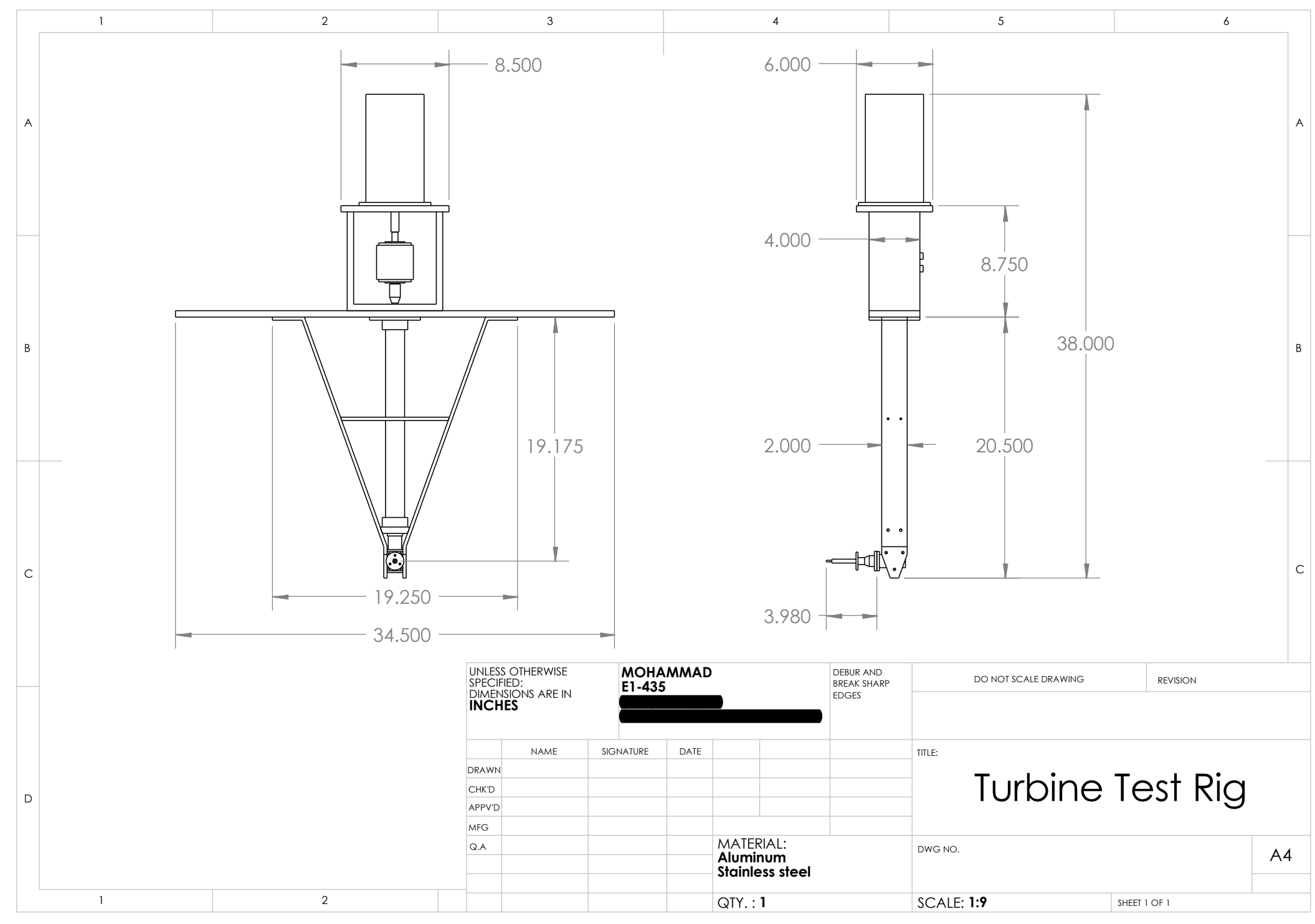




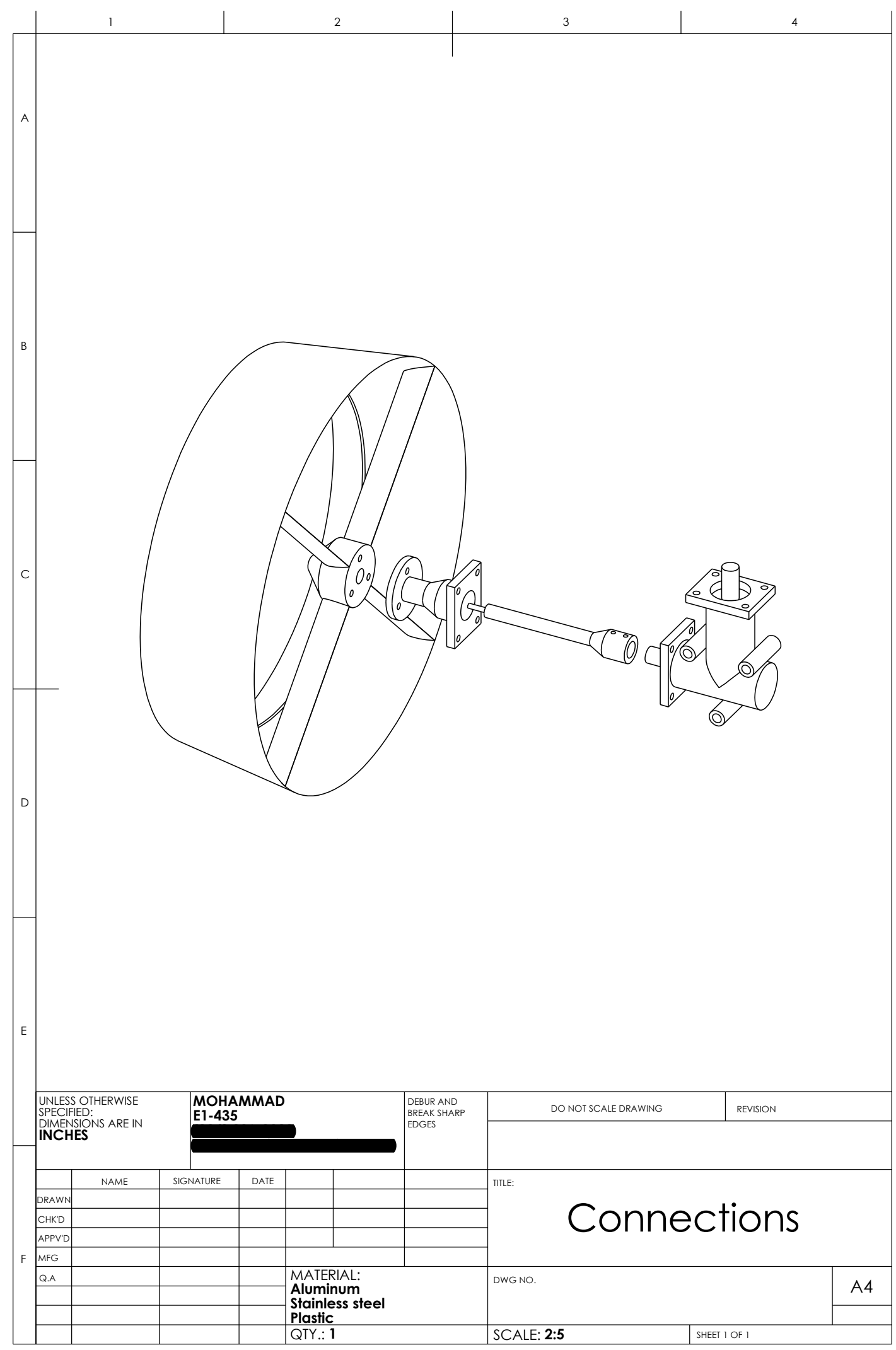




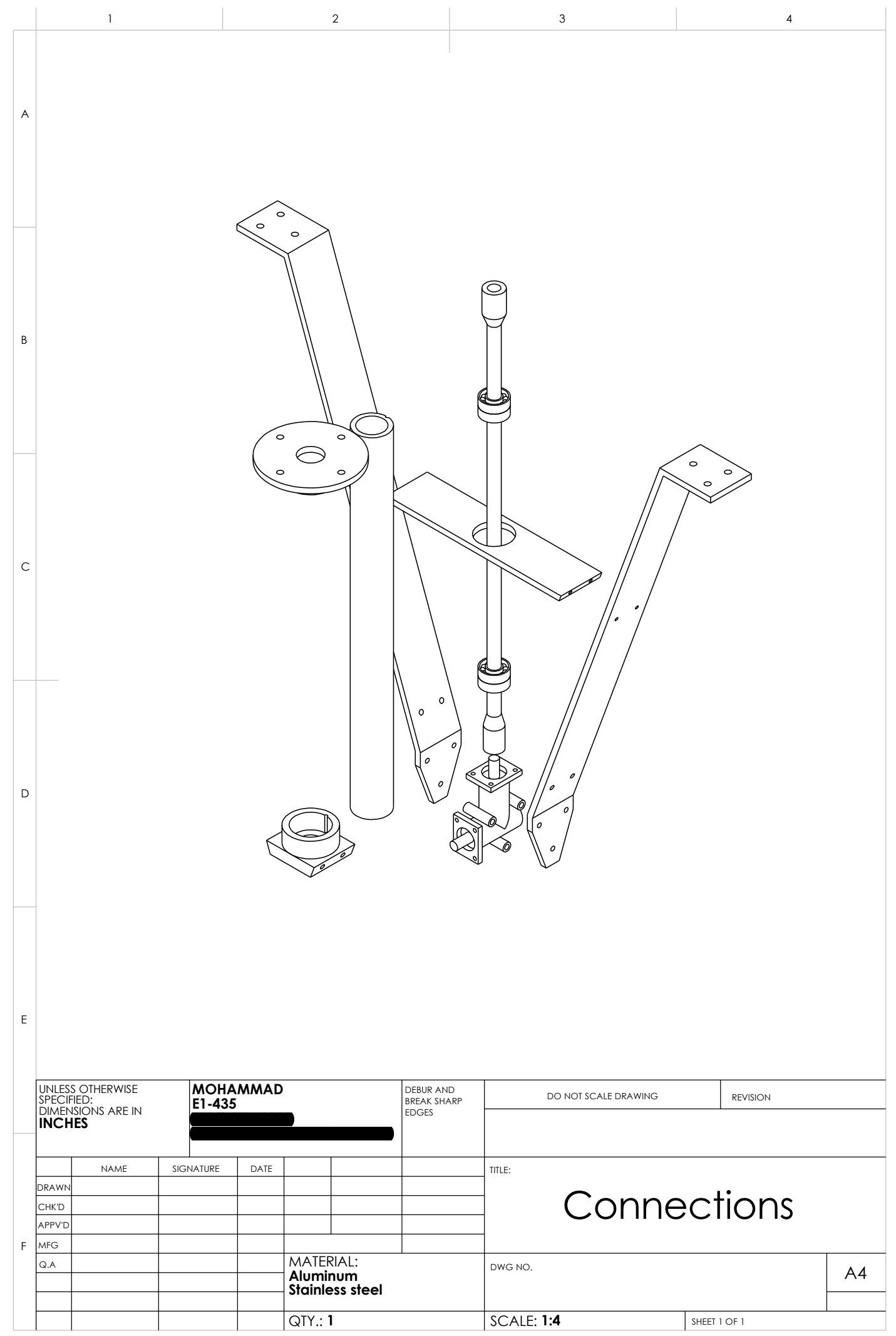




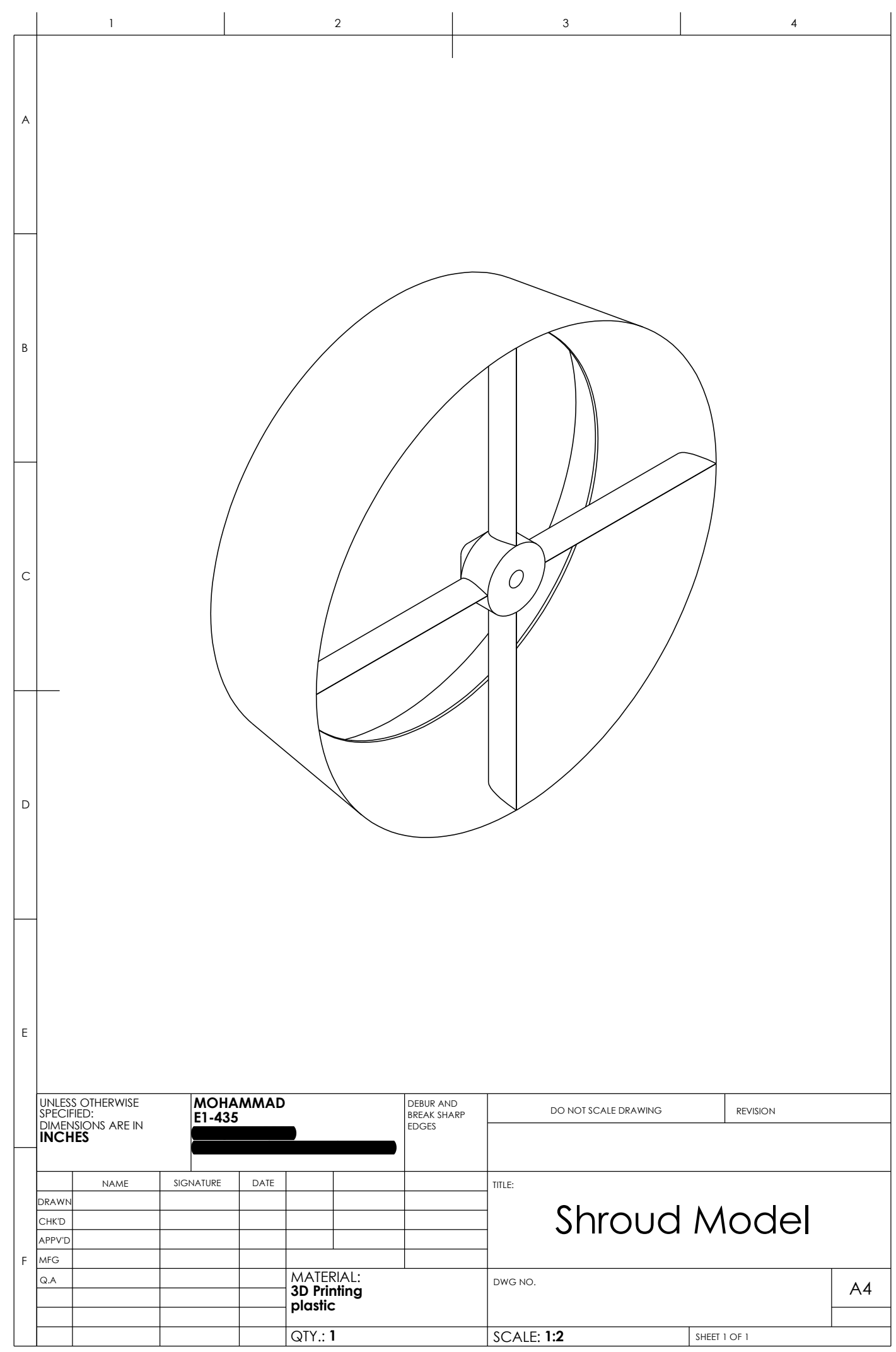




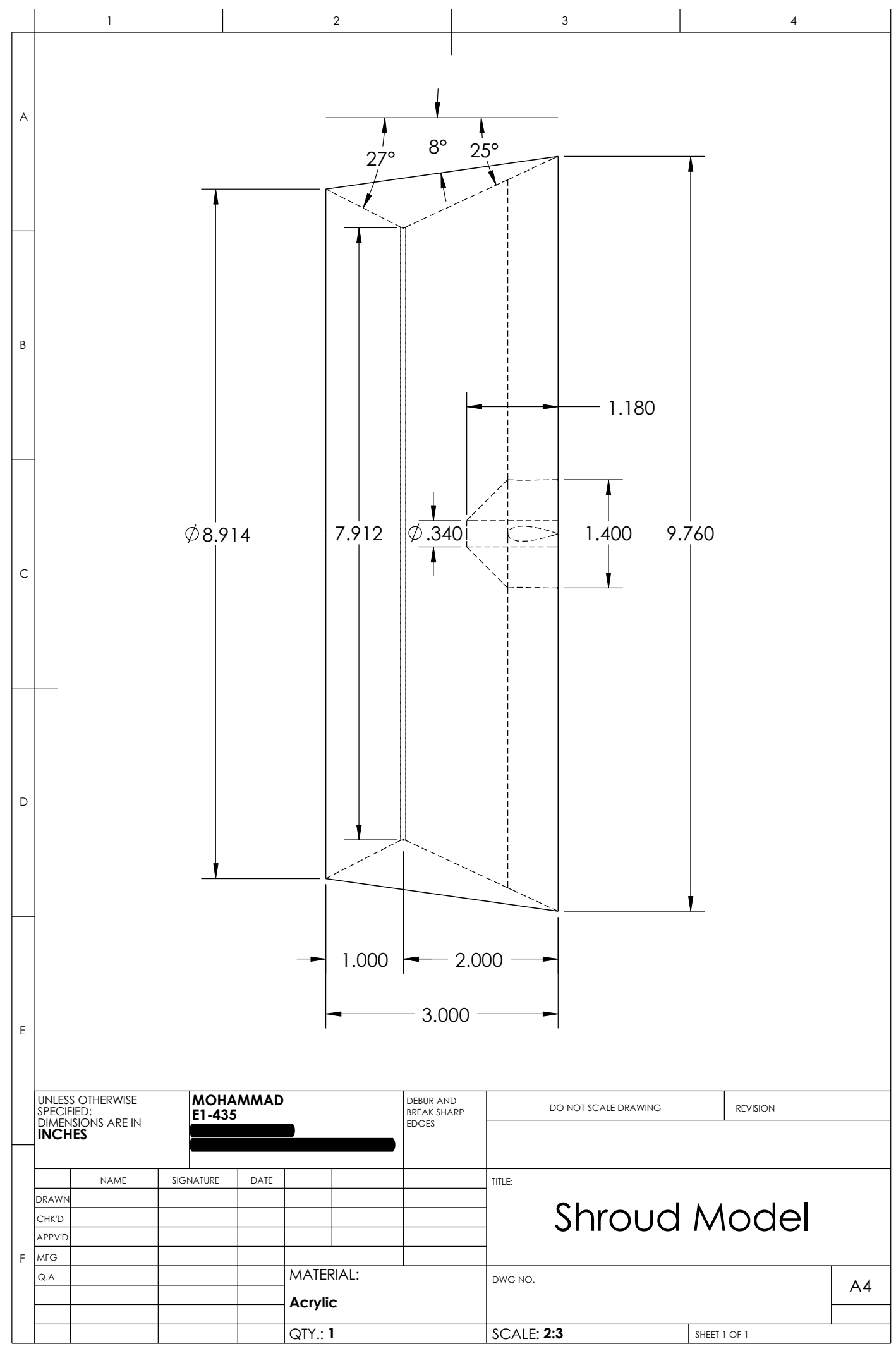




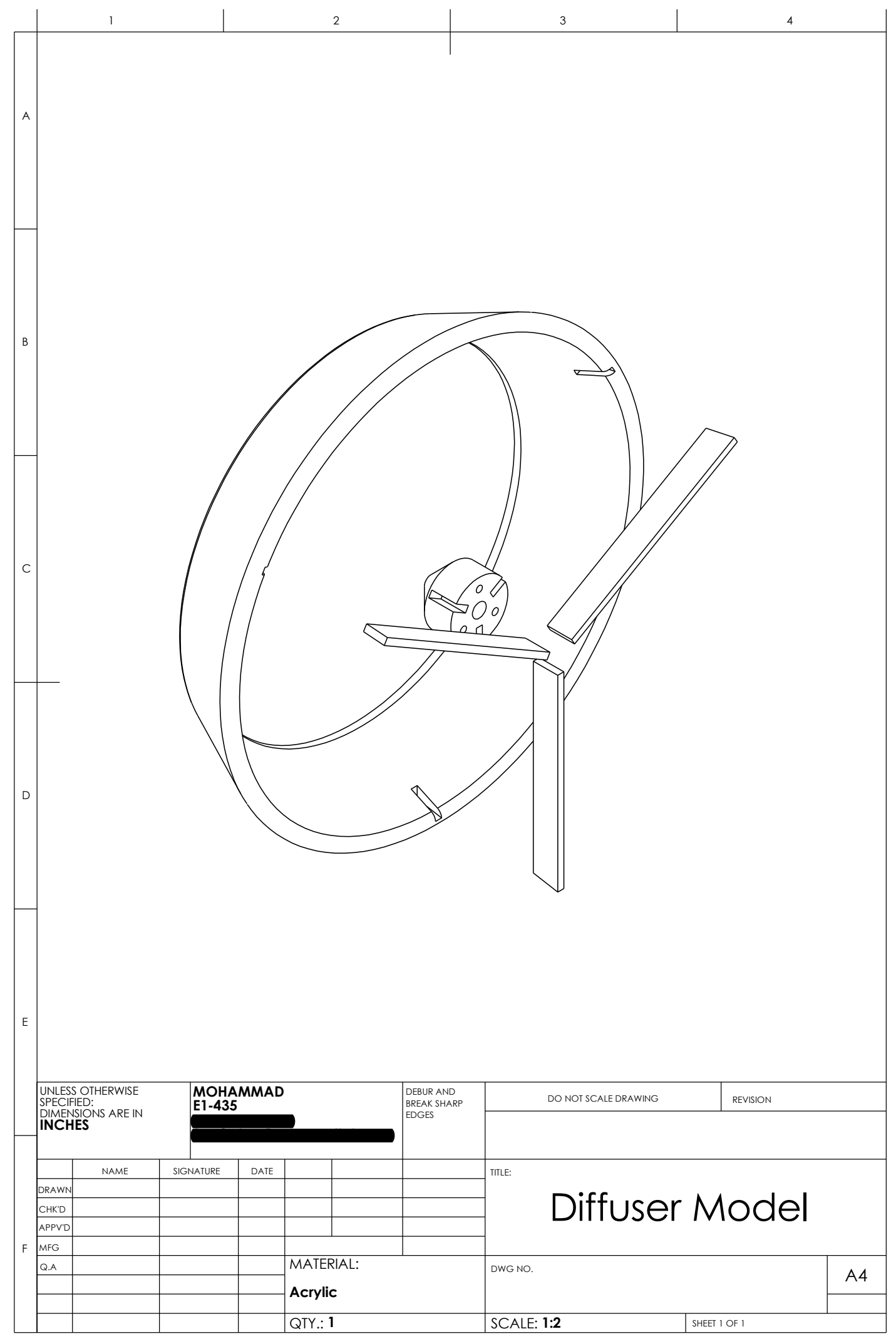




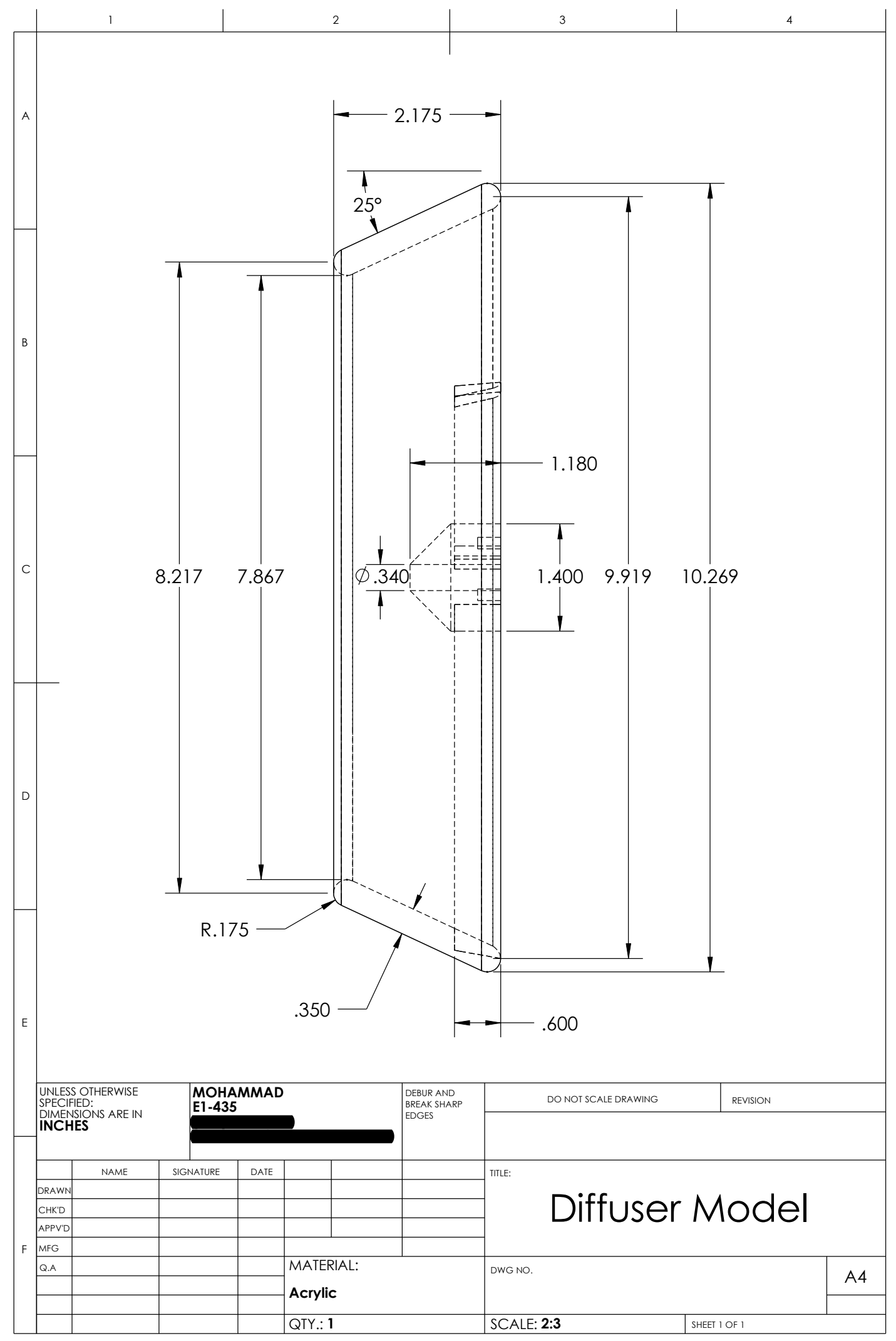




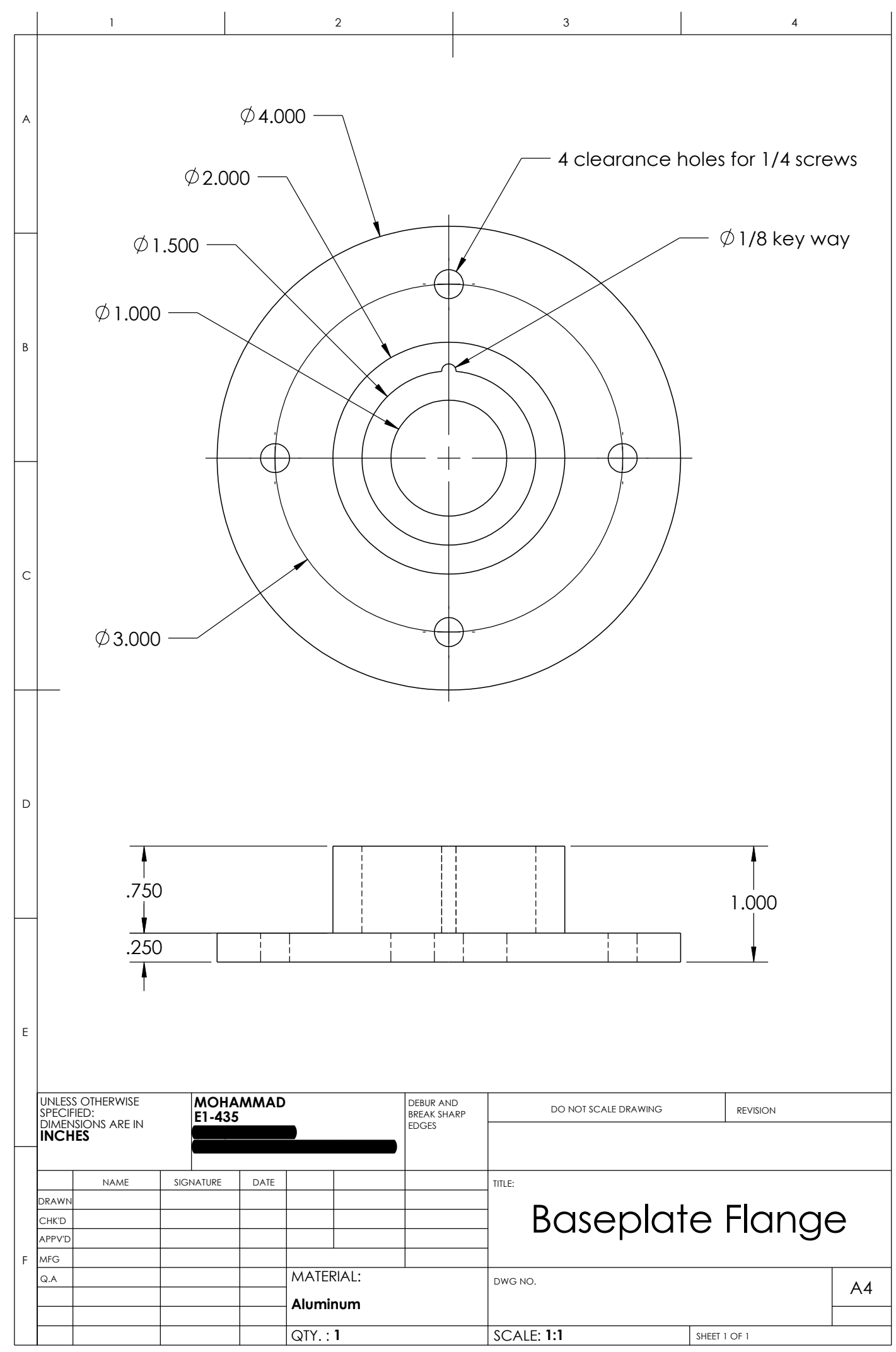




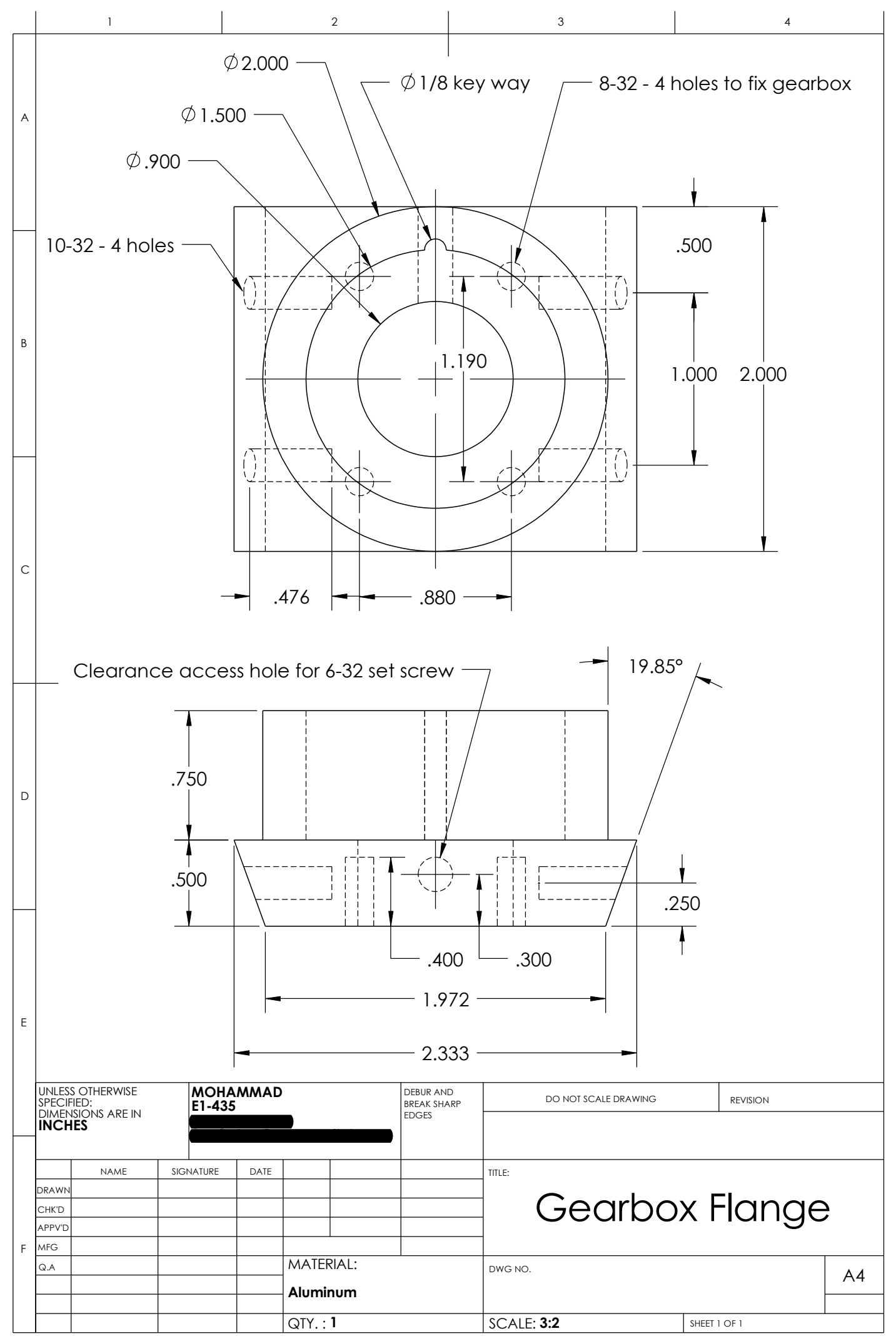




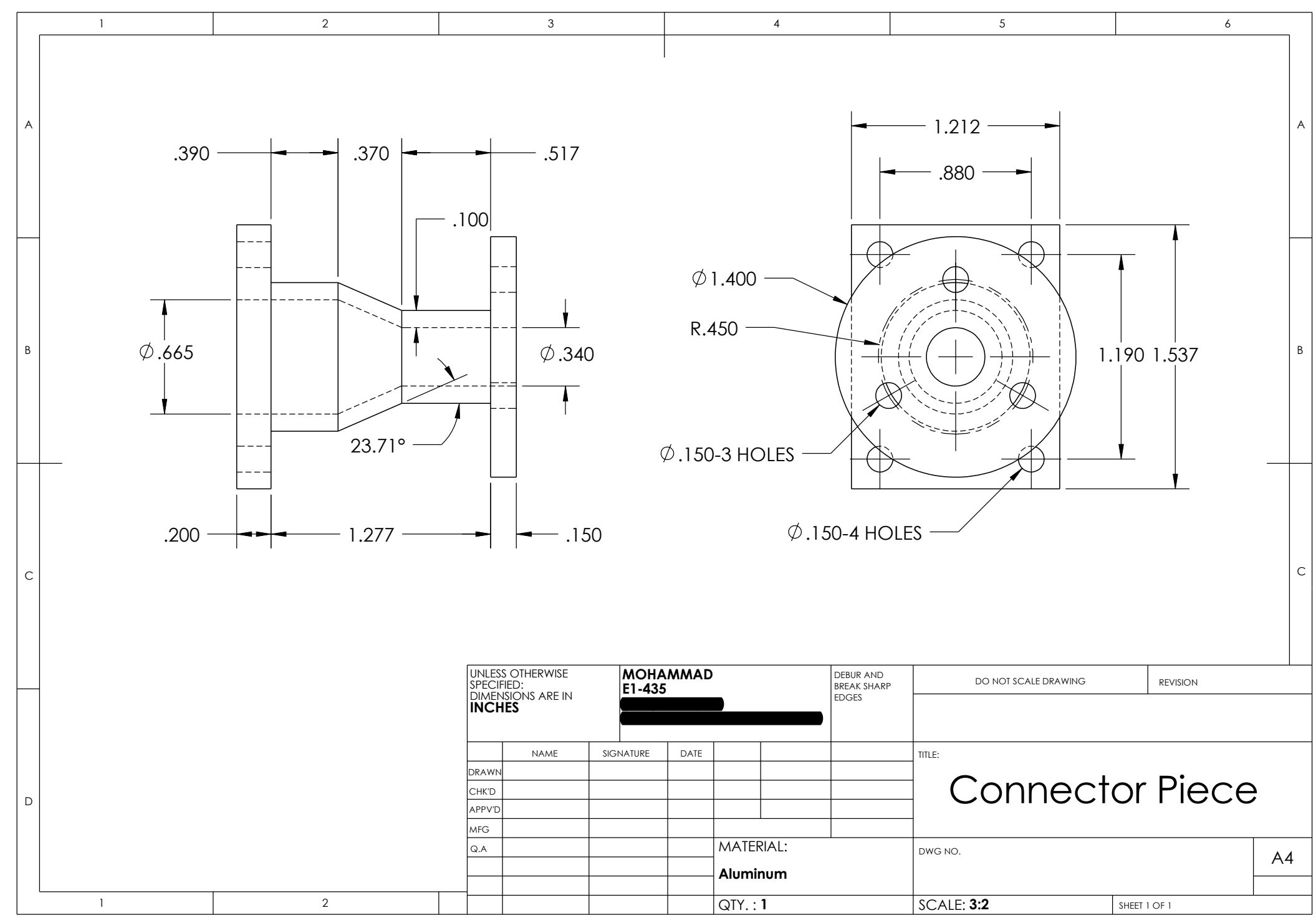




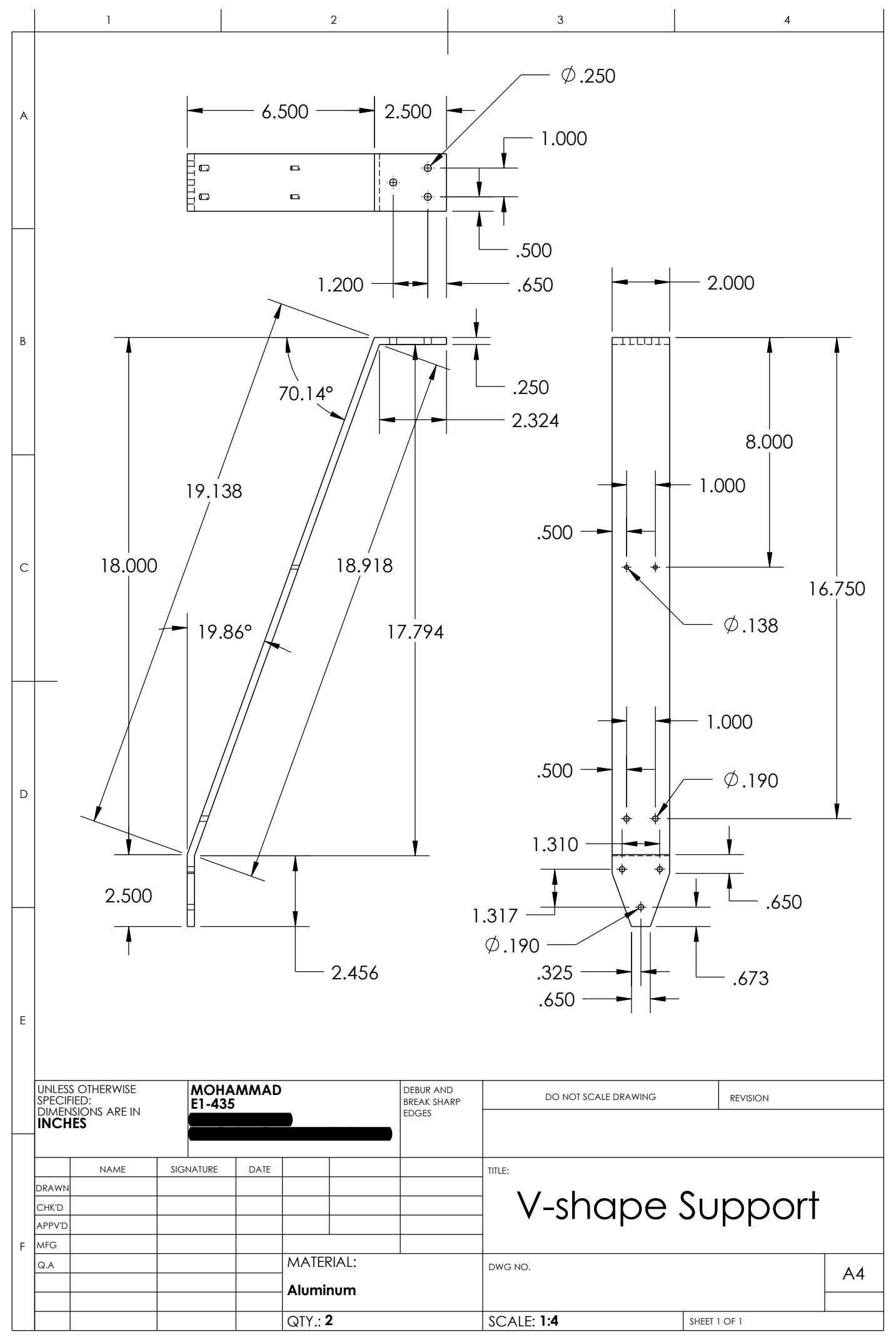




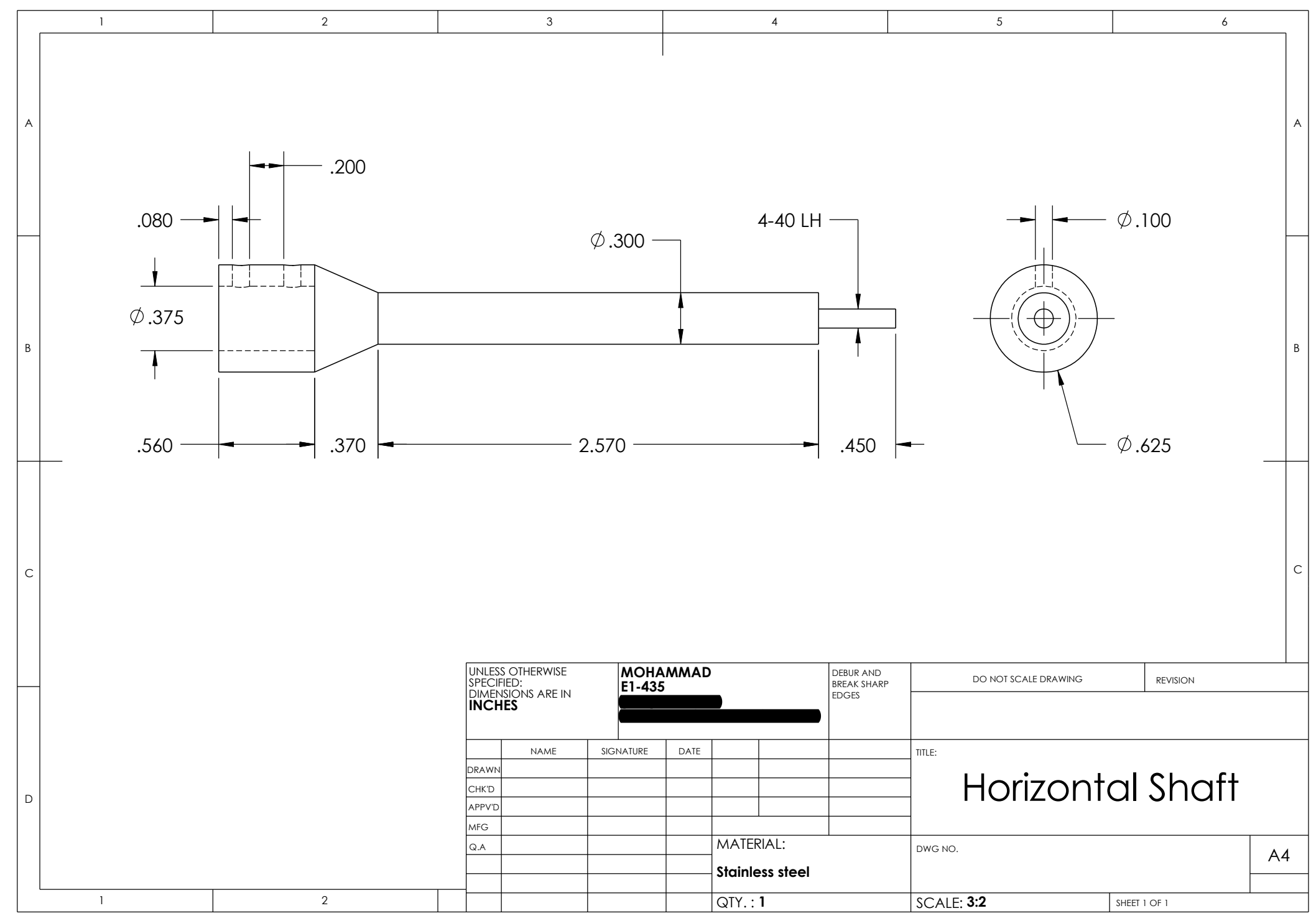




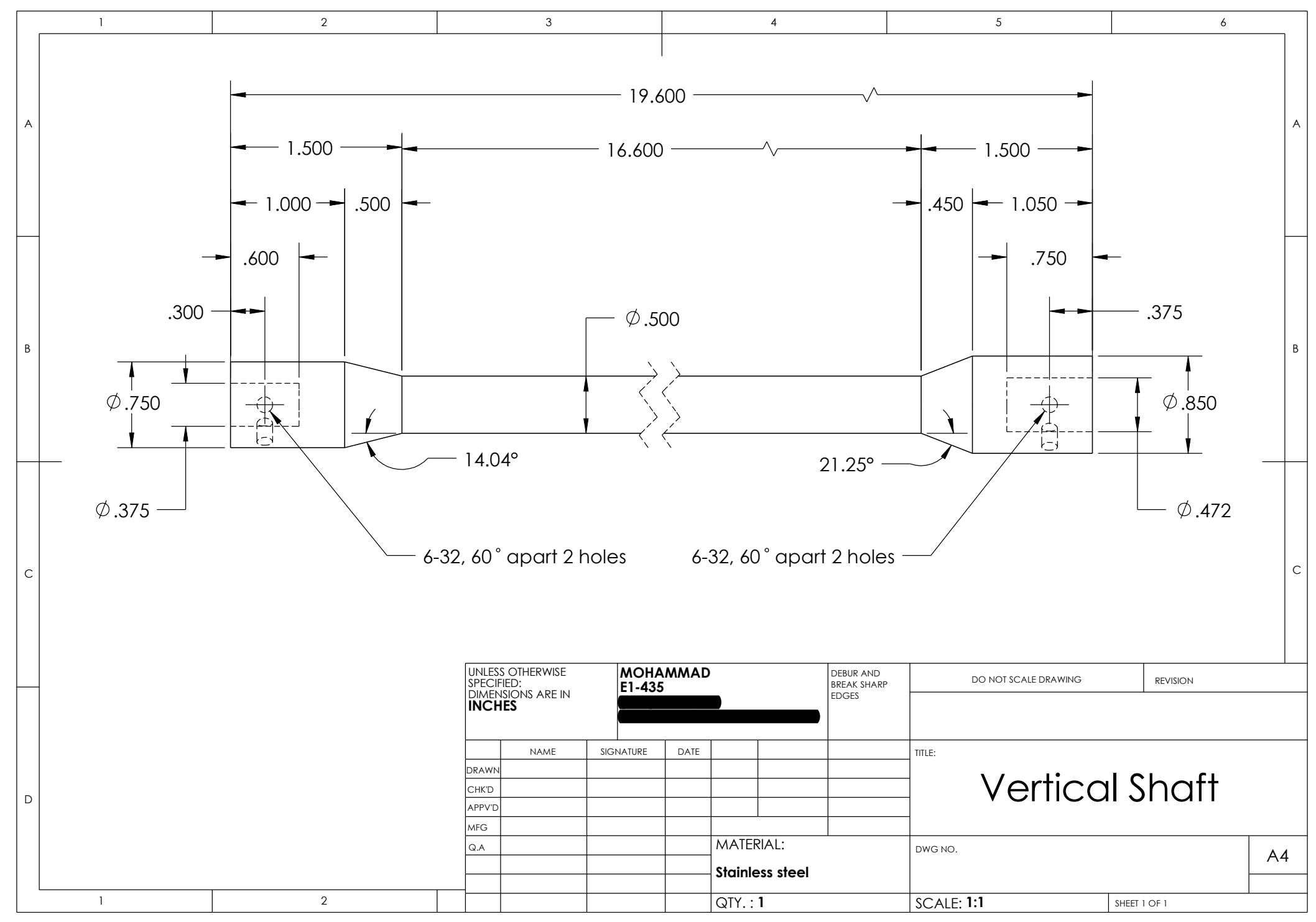




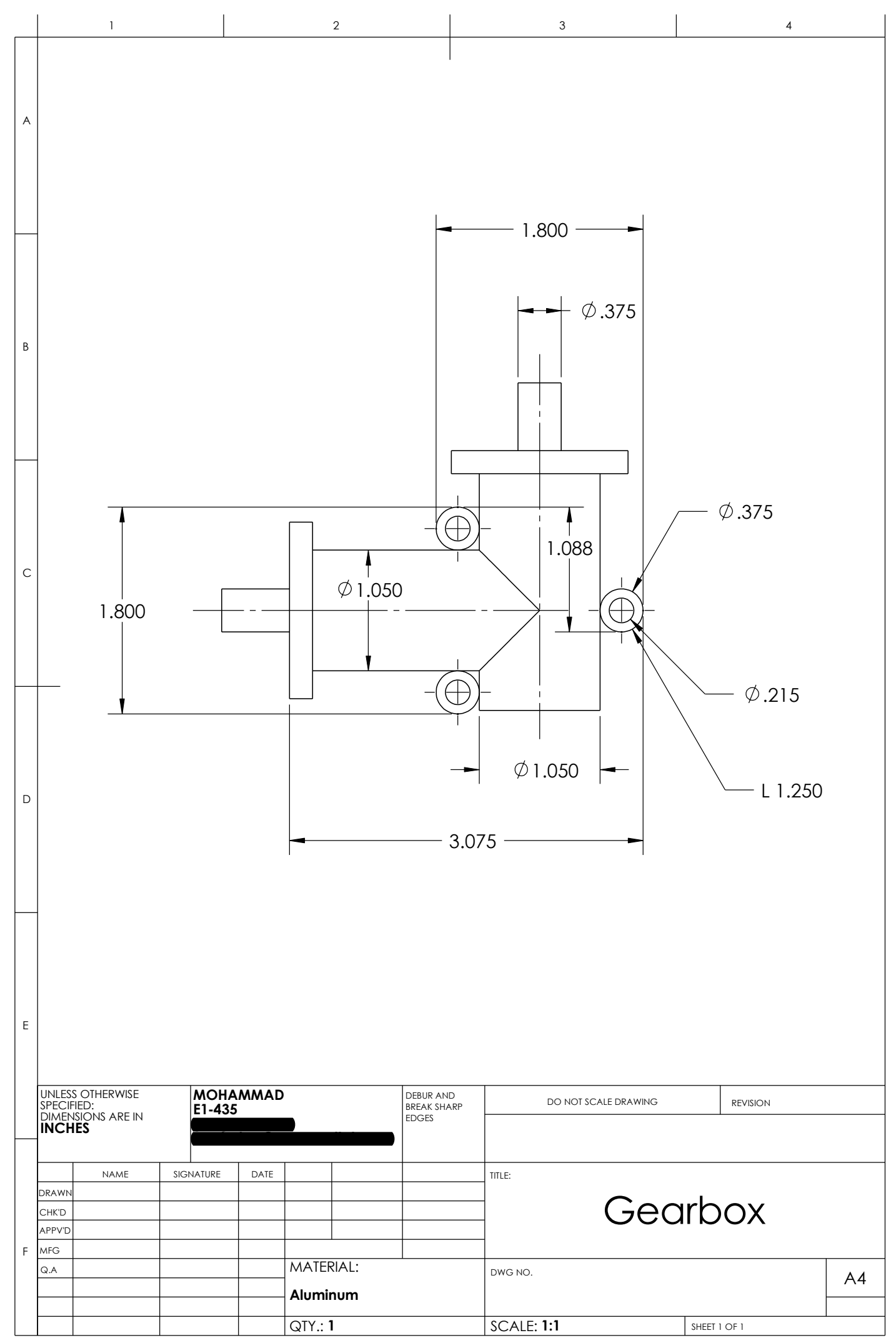

